\title{
DFI Computer Modeling Software (CMS)
}

\section{October 1979}

Prepared for:

\section{U.S. Department of Energy}

Energy Information Administration

Assistant Administrator for Applied Analysis

Under Contract No. WO-CR-03-7013

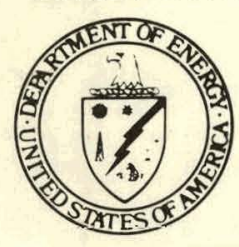

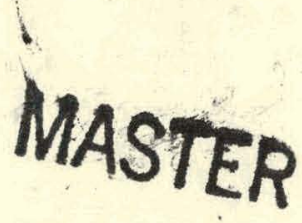




\section{DISCLAIMER}

This report was prepared as an account of work sponsored by an agency of the United States Government. Neither the United States Government nor any agency Thereof, nor any of their employees, makes any warranty, express or implied, or assumes any legal liability or responsibility for the accuracy, completeness, or usefulness of any information, apparatus, product, or process disclosed, or represents that its use would not infringe privately owned rights. Reference herein to any specific commercial product, process, or service by trade name, trademark, manufacturer, or otherwise does not necessarily constitute or imply its endorsement, recommendation, or favoring by the United States Government or any agency thereof. The views and opinions of authors expressed herein do not necessarily state or reflect those of the United States Government or any agency thereof. 


\section{DISCLAIMER}

Portions of this document may be illegible in electronic image products. Images are produced from the best available original document. 
This report was prepared as an account of work sponsored by the United States Government. Neither the United States nor the United States Energy Information Administration, nor any of their employees, nor any of their contractors, subcontractors, or their employees, makes any warranty, expressed or implied, or assumes any legal liability or responsibility for the accuracy, completeness or usefulness of any information, apparatus, product or process disclosed, or represents that its use would not infringe privately owned rights.

Released for printing January 15, 1980 . 


\section{MASTER \\ DFI Computer Modeling \\ Software (CMS)}

DOE/EIA-7013-1

Dist. Category UC-13

October 1979

Prepared by:

Edward G. Cazalet, Louis B. Deziel, Jr.,

Stephan M. Haas, Thomas W. Martin, Dale M. Nesbitt,

Robert L. Phillips

Decision Focus, Inc.

Palo Alto, CA

Under Contract No. WO-CR-03-7013

Prepared for:

\section{U.S. Department of Energy}

Energy Information Administration

Assistant Administrator for Applied Analysis

Office of Integrative Analysis

Long-Term Analysis Division

Washington, D.C. 20461
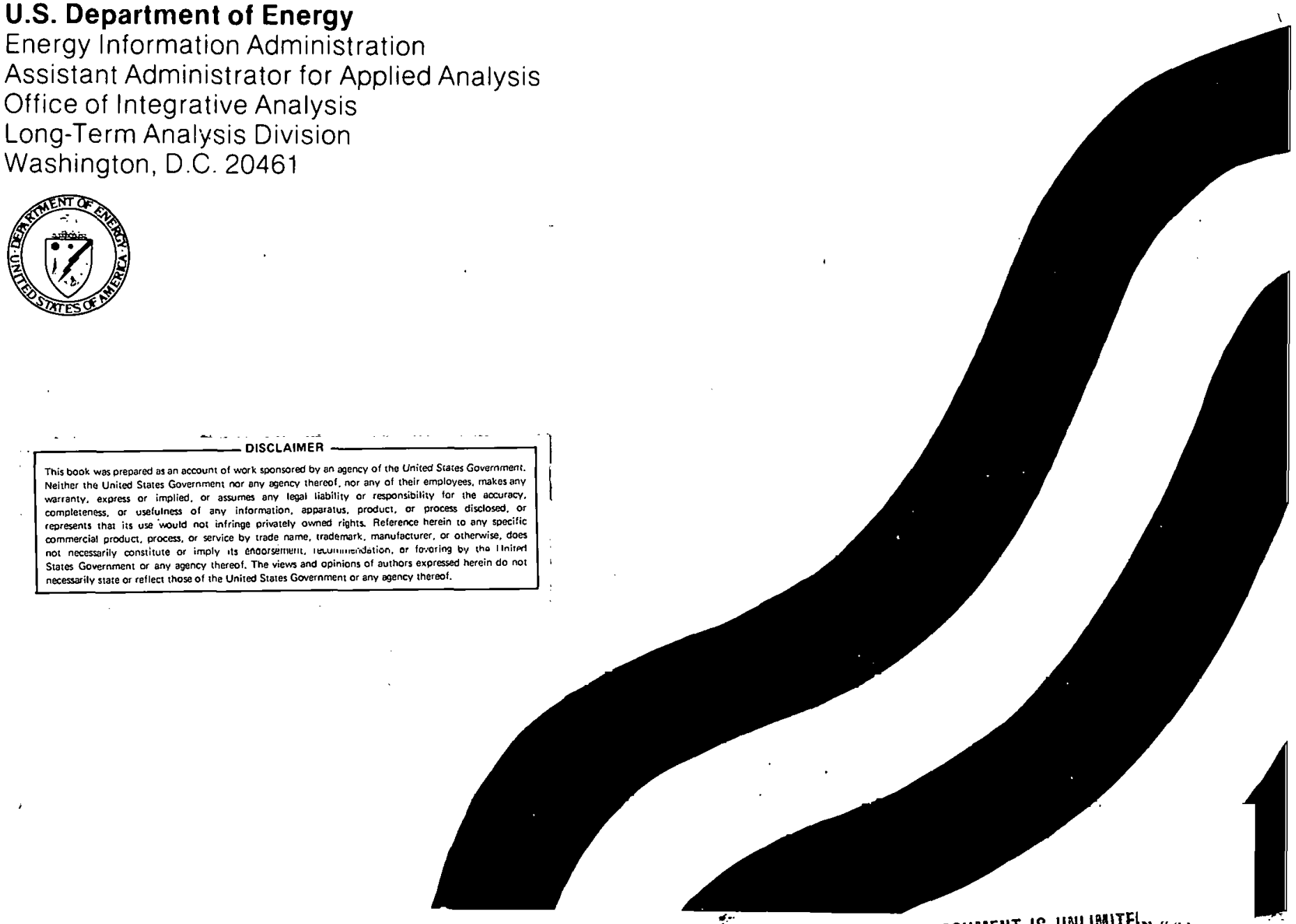

This book was prepared as an account of work sponsored by an agency of the United States Government. Neither the United States Government nor eny egency hereor, nor any of their employees. makos

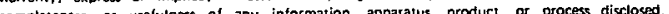

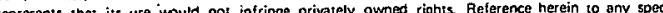




\section{DFI COMPUTER MODELING SOFTWARE (CMS)}

\section{ABSTRACT}

This document describes the data base management system used to create, edit and storé models, data and solutions for the LEAP system. The software is entirely in FORTRAN-G for the IBM 370 series of computers and provides interface with a commercial data base system SYSTEM-2000. 


\section{EXECUTIVE SUMMARY}

\section{The LEAP-EXEC Data Base System}

Version $1978-$ V1.

This document describes the present data base management system for the Long-term Energy Analysis Program (LEAP). It is based almost entirely on a contractor document "The DFI Computer Modeling Software (CMS) by Edward G. Cazalet ${ }^{+}$et. al. of Decision Focus, Incorporated (DFI). There have been minor user modifications incorporated and these are inserted in the text in Section 4, the users manual for the data base.

Generally speaking, the document is well-written and provides a good overview and some details of the computer software, and the authors are to be commended. It does not, however, completely document the computer code and many details can only be resolved by examination of the code.

+Edward G. Cazalet, Decision Focus, Incorporated; 1801 Page Mill Road, Palo Alto, California 94304 
I. GENERAL INFORMATION 1-1

A. PURPOSE OF THE DFI COMPUTER MODELING SOFTHARE $1-1$

B. OUTLINE 1-3

II. COMPUTER MODELING SOFTWARE OVERVIEW 2-1

A. BASIC'CONCEPTS 2-2

B. THE EXECUTIVE SYSTEM 2-16

C. THE DATA TRANSFER SYSTEM $2-25$

D. SYSTEM DESIGN 2-31

III. METHOPDOLOGY OF THE COMPUTER MODELING SOFTWARE

A. GENERAL PROPERTIES OF TREES 3-1

B. STANDARD TREE STRUCTURE 3-3

1. Branch and Node Naming/Numbering Conventions 3-4

2. Traversing the Tree 3-13

3. Storage versus Access Tradeoff 3-16

4. Sets 3-23

5. Square Trees 3-24

6. Single-Level Conditionality 3-27

7. Algorithm for Retrieving/Storing Values ' 3-41

C. DATA TRANSFER -- FILLING A LABELLED COMMON - 3-44

IV. USING THE COMPUTER MODELING SOFTWARE

A. USING THE EXECUTIVE SYSTEM

1. Introduction '. 4-1

2. System Tables 4-2

a. INPUT/OUTPUT TABLE 4-3

b. Name Conditionality 4-6

3. Catalog of Executive Commands 4-10

a. Model Retrieva1/Creation Command 4-12 i. GMOD $4: 13$ 
b. Workspace Manipulation Commands 4-14

i. SIZE $4-15$

ii. INIT 4-17

iij. CHANGE $4-18$

iv. $A D D \quad 4-22$

v. DEL 4-23

vi. EXCH 4-24

c. Workspace Display Commands 4-25

i. SET $4-26$

41. DISP 4-27

d. System Table Commands 4-29

i. GSTB $4-30$

ii. PSTB $4-32$

iii. RSTB 4-33

e. Hierarchical Table Commands 4-36

i. GTAB 4-37

ii. NEXT 4-38

iii. RTAB $4-43$

iv. PTAB , .4-45

f. End of Session Conmand 4-46

i. END 4-47

4. Accessing the Executive 4-48

3. DATA TRANSFER SYSTE!M USE $\quad 4-49$

1. Labelled Common Design 4-49

2. LCS and LCO Tables 4-54

3. Main Program 4-62

4. Caliing FILL and STOR 4-64 
V. EXECUTIVE PROGRAMMER'S GUIDE:

A. PROGRAMMING CONVENTIONS

B. THE PRIMARY AREA

c. CONTROL AND PARSING

1. Subroutine PARSE

2. An example of the Flow of Control

D. DATA BASE INTERFACE

1. Traversing the Hierarchy

2. Updating the Hierarchy

a. Accessing Data

b. Adding Structure

$5-33$

E. APPENDIX

$5-39$

F. BIBLIOGRAPHY

VI. DATA TRANSFER SYSTEM - PROGRAMMERS GUIDE

A. INTRODUCTION 6-1

B. THE SOFTIN ALGORITHM 6-1

1. Overview : 6-1

2. Data Storage 6-3

. 3. Reading the System Tables (BLDTBL/FINTBL) 6-8

4. Building the Offsets (BLDOFF) 6-13

a. Traversing the System 2000 Unload File 6-13

b. Establishing the Offsets (BLDOFF/OCOMP) . 6-18

c. Updating the Offsets (BLDOFF) 6-34

d. Additional, Offset. Information (FINOFF) 6-43

5. Placing Values in the RD Vector (BLDD/DCOMP) 6-43 
C. FILL/STORE ALGORITHM

1. Initialization Steps in Fill

2. Filling from a One Level Set

3. Establishing the Clock

4. Establishing Conditionality Information for Clock Dimensions

5. Determine Whether Any Values Need Be Read into the Labelled Cormon

6. Transferring a Single Value From the RD Vector to the Labelled Cormon

7. Incrementing the Clock to Determine Whether to Transfer Another Value

D. THE SOFTOUT ALGORITHM (SOFTOU)

1. Overview of the Two Options

2. Similarity of REPLD to BLDD

E. SELECTIVE FILLING/STORING (SFILL/SSTOR)

1. Introduction

2. Algorithm

3. Extensions

F. UTILITY ROUTINES

6-102

G. HELPFUL HINTS 


\section{CHAPTER I}

\section{GENERAL INFORMATION}

\section{A. PURPOSE OF THE DFI COMPUTER MODELING SOFTWARE}

Computer modeling is most effective when it is used to bring together information from diverse sources to provide timely insights to decision makers facing complex problems. Unfortunately computer modeling is usually time consuming and expensive. The purpose of the DFI Computer Modeling Software (CMS) is to reduce the time and cost required to build and use computer models and thius to allow more time to improve their quality.

The approaches to computer modeling embodied in this software have evolved from the problem solving experience of the DFI. staff. Most frequently, our models are built during the analysis of specific decision problems for clients. As such, our models must be constructed, run, documented, and communicated in a relatively short period of time -- say one to six months. Some of these models have been used only for a short period of time to evaluate the specific set of alternatives for which they were designed while others have enjoyed a longer life because of their more general applicability. These longer lived models have been used repetitively and improved over many years in the course of ongoing analyses of related decision problems. 
As a result of our experience in computer modeling, we have come to the following conclusions which have contributed to the design of this software:

1. The most expensive and time consuming aspect of analysis and model building is communication among the participants -- clients, experts, and analysts. Yet it is precisely from this communication that effective models arise. Thus we have sought to develop a flexible structure within which models can be embedded that allows models to evolve according to the needs of the project. This is to be contrasted with the largely unsuccessful approach of attacking a decision problem with a pre-existing model whose implementation is so rigid that it cannot be changed in a timely or cost-effective fashion.

2. Much of the time required to develop a new model is spent managing input data and model results. The DFI-CMS relieves the modeler of the need to develop input and output format statements and file handling programs. By structuring the input and output data in the, form of two dimensional tables, the CMS provides a flexible method to input, edit, store, and display data as well as to place the data into the model logic upon request.

3. We have found it extremely effective to define and use a set of formal programing conventions for writing model code and for interfacing that code with the data base management system. Formalizing the programming conventions allows other analysts easier access to existing model logic, thus allowing pre-existing model logic to be regarded as capital. This is to be contrasted with the frequent practice of beginning model logic 
development from scratch on every new project even though much of the logic developed on previous projects is "transportable."

4. We have found that $\mathrm{clients}$ and experts tend to be averse to direct interaction with computer models. The reason arises in part from the fact that the computer demands that the user speak in its language, which is unnatural to the user. The DFI-CMS partically offsets this difficulty by decoupling the data input/results ouput procedures from the model running procedures and by specifying a very natural language for data input/results output--two dimensional tables with headings, row names, column names, and explanatory footnotes.

The purpose of this document is to describe the DFI Computer Modeling Software (CIS) in detail. The document is intended as a basic reference for teaching new users about the DFI-CMS as well as for its ongoing use and development. In this volume we will not describe specific models or how to build models but instead will describe how the software works and how models are embedded within it.

\section{OUTLINE}

This document is organized into six chapters. Chapter I contains an introduction and outline. In Chapter II, we introduce and motivate the basic concepts of the DFI Computer Modeling Software. The discussion is quite general but gives the reader a rather thorough description of the concepts underlying the system. This discussion is conducted around a simple example.

Chapter III discusses the methodology of the data transfer system in some detail. The discussion is intended to communicate the details of the system without burdening the reader with FORTRAN variable 
names, logic flow charts, or algorithms.

Chapter IV then outlines how to use the system. It contains only enough technical detail about the implementation of the system to describe the procedure a user must follow to interface his model with the software. There are three sections in this user's guide chapter -one for the Executive system, one for the Data Transfer System, and one for running a model once it is embedded in the system.

Chapter $V$ contains a detailed description of the Executive System and is intended as a programer's guide. This chapter is not light reading and is intended for those who'must understand the details of the Executive algorithms.

Chapter VI contains a detailed description of the Data Transfer System and is intended to serve as a programmer's guide. Like Chapter V, this chapter is quite detailed and is intended for those who must operate or modify the Data Transfer System. 


\section{CHAPTER II}

COMPUTER MODELLING SOFTWARE OVERVIEW

The use of quantitative models represents a highly structured approach to problem solving. In order to construct a model, one must specify a set of equations and how those equations interact. In so doing, the modeler has implicitly specified how the parameters of those equations (f.e. the input data) inter-relate. Thus, the structure of a given model implies a structure for the input data it requires. Because we generally wish to change the details of a model's structure during the course of an analysis, it is necessary to develop a comprehensive and flexible data structure that can be quickly and easily adapted to a wide variety of model structures.

When large models and/or large data bases are involved, the problem of data management becomes central to the successful and timely application of the model. We have found that even when models themselves are not large, data requirements can still become sizable because we wish to define and compare a large number of sensitivity runs both in terms of input data and output data (results). Thus we have expanded the definition of data to include both input and output data sets. This expanded notion of model data is important because as the model is applied to different decision problems, the model output of interest is likely to change. Thus flexibility to provide various levels of output detai? 
must be provided. Similarly as a model "grows,' values which were previously given exogenously as input data may now be calculated internally. Hence there is a basic need for a flexible data base management system.

This expanded notion of the data base and how we relate it to the model is shown graphically in Figure II.1. The model draws values from the data base, transforms them, and replaces them. The user interacts with the data base by defining input values and by displaying and inspecting both inputs and results. The data base management system we have developed provides a means for achieving these interactions in an efficient manner.

The remainder of this section is divided into four parts. The first introduces and explains the basic concepts behind the DFI computer modeling software. The next two sections provide an introduction to the main components of the software (the Executive. System and the Data Transfer System.) The final section explains how these components are linked together in the actual.computer implementation.

\section{A. BASIC CONCEPTS}

To determine the data base structure that is most appropriate to a particular problem, we look to the structure of the corresponding model to guide us. We have consistently found that the variables and parameters in a model can be organized in terms of several categories which we call dimensions. For example, one dimension of a regionallydisaggregated model would be the "location" dimension since some 


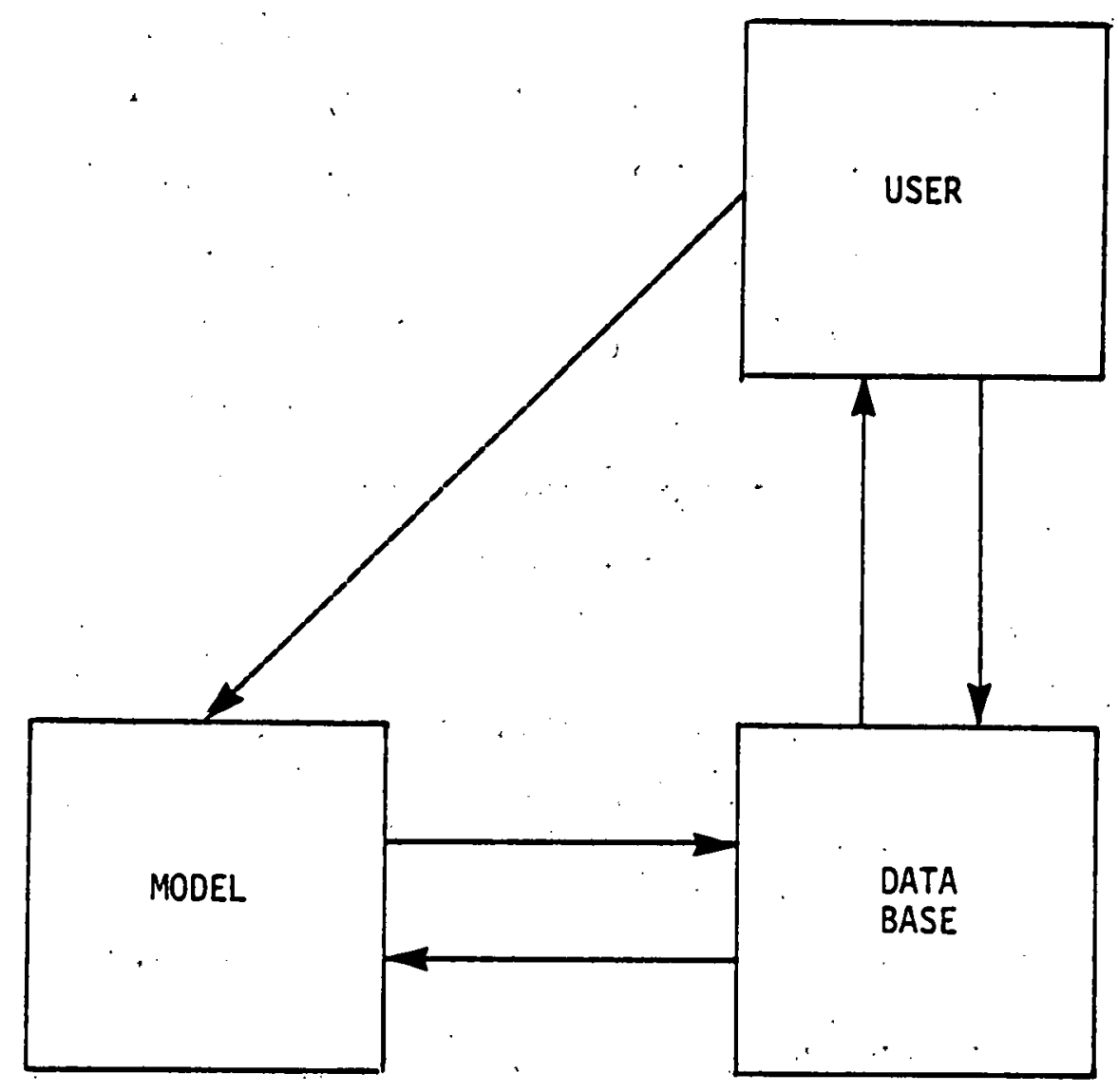

Figure II.1 MODEL/OATA BASE/USER INTERACTIONS 
variables must differ by location in order for regional disaggregation to be justified. Another dimension might be time. Some variables might be described by vectors over time while others might not. Given this dimensional categorization, we can view the model data (inputs and results) as contained within an $n$-dimensional cube where $n$ is the number of dimensions specified. Each axis of the cube corresponds to a dimension and the length of the cube in a given direction depends on the size of the corresponding dimension. For instance, if eight regions had been defined and indexed 1-8, the "region" axis of the cube would be eight elements long.

To illustrate the concept of the n-dimensional cube, we will consider the simple model represented by the following equation:

$$
P V=\sum_{i=1}^{N} \text { cf }_{i}\left(\frac{1}{1+r}\right)^{i}
$$

This model computes the present value (PV) of a stream of cash flows $\left(c f_{i}, i=1, \ldots, N\right)$ over $N$ time periods at the interest rate $r$.

We notice in the present value equation above there are two basic types of variables -- those that vary over time $\left(\epsilon_{j}\right)$ and those that do not ( $N, r, P V)$. Hence we can think of time as one of the dimensions of this simple model. If we index the variables in the model as follows:

1: present value (PV)

2: number of time periods $(N)$

3: interest rate $(r)$

4: cash flow $\left(c f_{1}, \ldots, c f_{N}\right)$, 
we can write the entire data base in the form of a two dimensional cube, i.e.,a matrix, as shown in Figure II.A.1. Notice that each element of the data set, both input and output variables, can be specified by a pair of indices,i.e., the normal two-dimensional matrix indexing method. However, the storage and conceptual inefficiency of this method is obvious.

\section{Attribute}

1. Present Value

2. Number of time periods

3. Interest Rate

4. Cash Flow
Time

$\begin{array}{rrrrr}1 & 2 & 3 & 4 & 5 \ldots . \\ N & N & N & N & N \ldots . \\ r & r & r & r & r \ldots . \\ c f_{1} & c f_{2} & c f_{3} & c f_{4} & c f_{5} \ldots c f_{N}\end{array}$

Figure II.A.1: MODEL DATA 
The data for this model can be more efficiently represented in the form of a tree whose levels correspond to the two dimensions of the model. Such a tree is shown in Figure II.A.2. The leftmost branch at the top level, which corresponds to the time dimension, contains all those values which are independent of time ( $P V, N$, and $r$ ). The rightmost branches $1, \ldots, N$ emanating from the top level correspond to timedependent values. In the bottom level of the tree, denoted the attribute level, the three time-independent variables are seen to emanate from the leftmost node and the individual cash flow elements are seen to emanate from the other nodes.

As can be seen in the figure, the tree contains one path for each element of data. Thus, each element is uniquely specified in terms only of the dimensions upon which it depends -- those upon which it does not depend are suppressed.

This tree representation provides a much more efficient means of storage than the two-dimensional matrix. Furthermore, the tree contains a useful graphical representation of the underlying model structure. For example, the tree structure in Figure II.A.2 shows clearly that the discount rate $r$ is assumed to be constant over time. If one wished to change this assumption, he would change the simple model above to the following form:

$$
P V=\sum_{i=1}^{N} \mathrm{cf}_{i} \prod_{j=1}^{i}\left(\frac{1}{1+r_{j}}\right)
$$




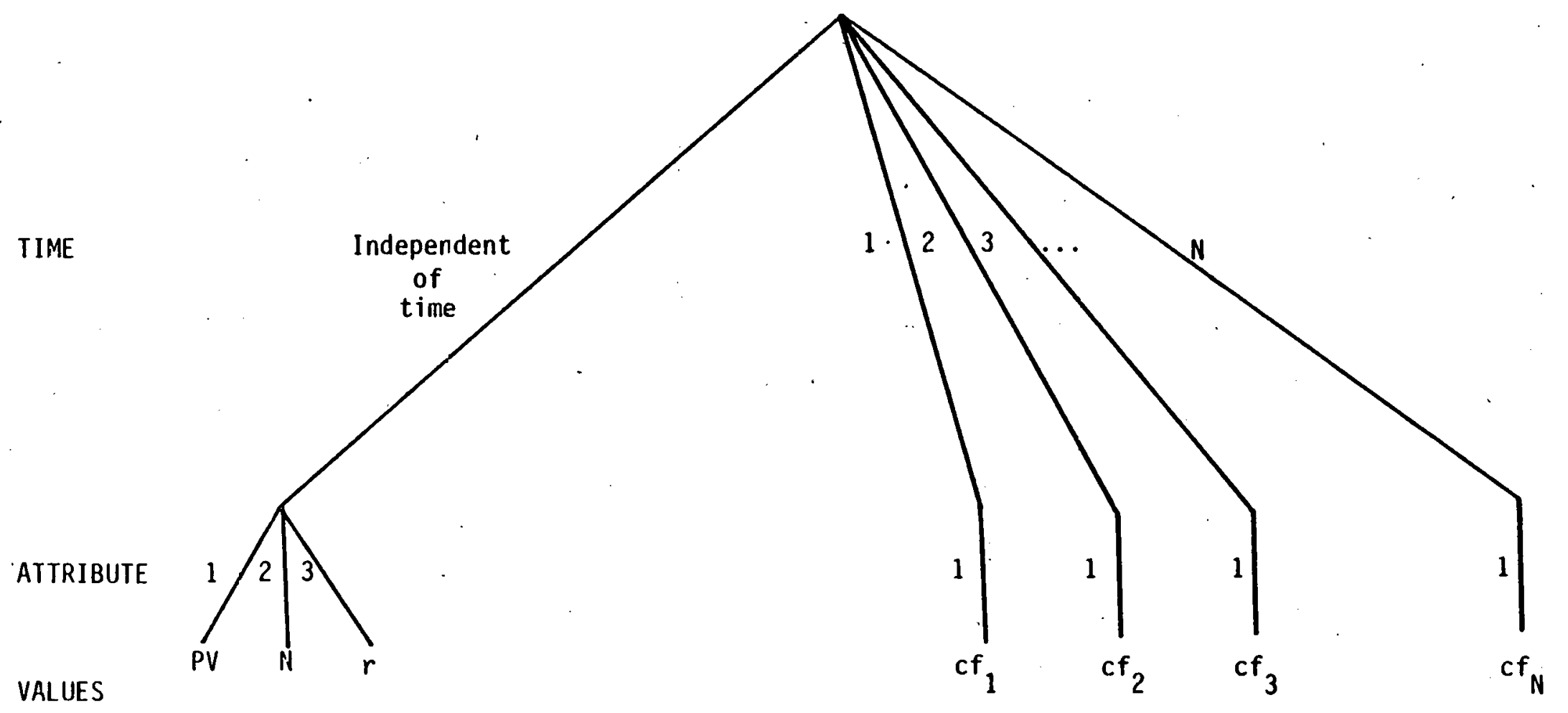

Figure II.A.2 TREE REPRESENTATION 
and the tree in Figure II.A.2 would be changed as shown in. Fjgure II.A.3. The two figures illustrate how the assumptions inherent in a model become obvious when its data base is displayed in dimensional form, which we see as a useful communication tool in its own right.

Returning to the tree representation in Figure II.A.2, we can now visualize an efficient data storage and retrieval method. Suppose we were to number the terminal branches on the tree from left to right as follows:

Table II.A. 1: TERMINAL BRANCH INDEXING

\begin{tabular}{ll|l|l|l|l|l|l} 
Terminal Branches & 1 & 2 & 3 & 4 & 5 & $\ldots$ & $\mathrm{N}+3$ \\
\hline Variable & PV & $\mathrm{N}$ & $\mathrm{r}$ & $\mathrm{cf}$ & $\mathrm{cf}_{2}$ & $\ldots$ & $\mathrm{cf}_{\mathrm{N}}$
\end{tabular}

This indexing of the terminal branches would allow us to access each of the input and output variables in the data base uniquely and would eliminate the need for storage of redundant information or for defining unused storage locations. We can thus think of the tree itself as an indexing or pointer scheme with respect. to the terminal branches where the values actually lie. Recognizing this, we need only develop a digital representation of the tree and use it to access the individual elements of data as needed. The details of this access system, which we call the Data Transfer System, will be described in Section C of this chapter. 
TIME

\section{ATTRIBUTE}

VALUE

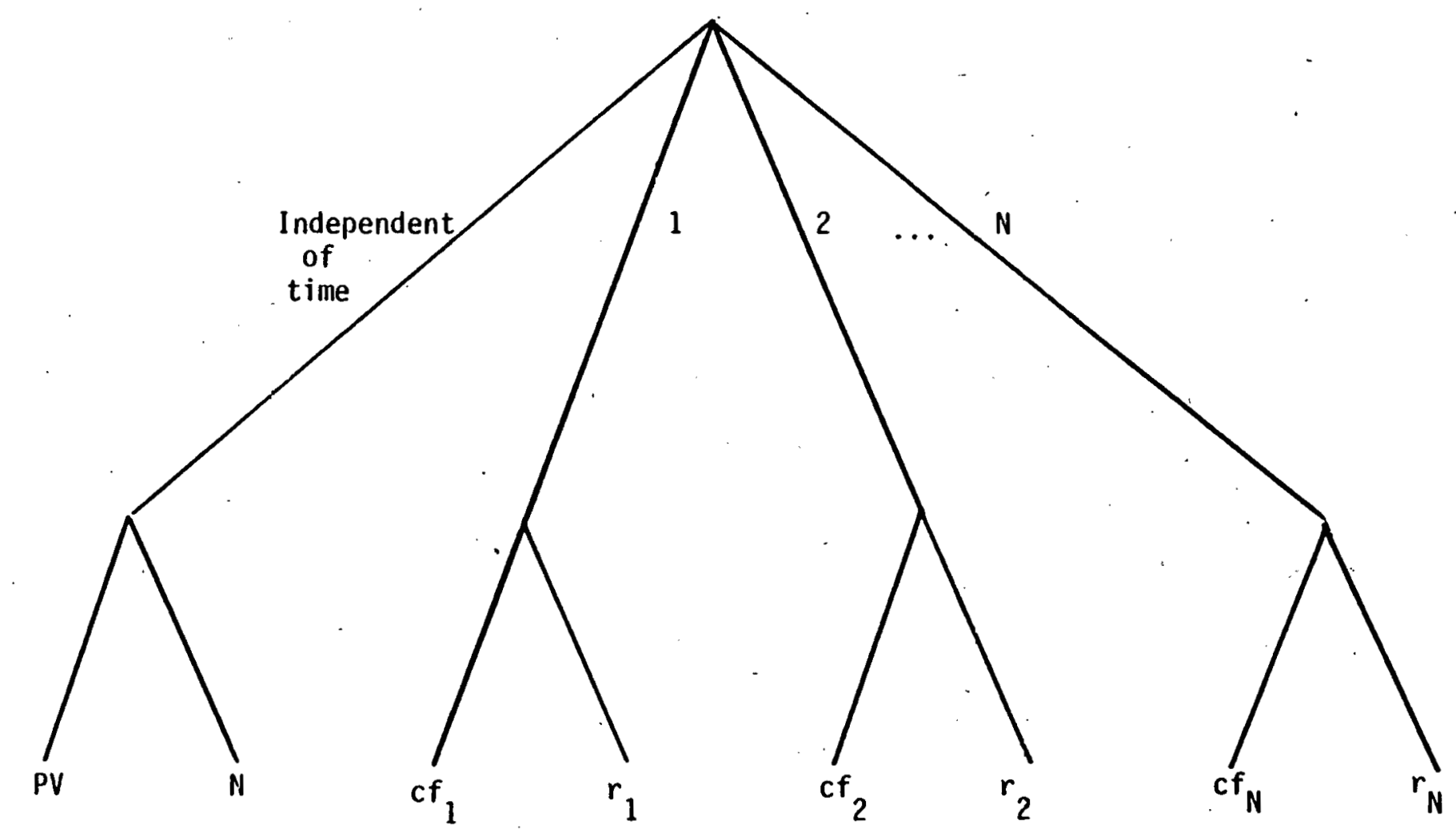

Figure II.A.3 TIME-VARYIMG IMTEREST RATES 
The preceding discussion illustrates the concepts of dimensionality and "tree storage" in a very simple case. We will now extend these concepts to the more general case. As a first step suppose we have developed a model that has four variables which vary over 5 time periods and three regions. The three dimensions indicated for such a model would be:

- Region

o Time

- Attributes.

If we wished to use a cube to describe the data base for this model, the three-dimensional cube in Figure II.A. 4 would be required. We can think of the cube as having a storage position at each of the intersections of lines in the figure. As with the previous case the natural indexing of the cube (ie. the "Euclidean" indexing) uniquely identifies each element. Thus, the vector $(2,3,1)$ indexes the value of the second variable in the third time period in region 1.

Notice that the lowest dimension in this model is called the attribute dimension as in the previous example. The attribute dimension is special for a number of reasons. First, by convention, it will aiways be the lowest dimension in the hierarchical data tree. Secondly, all values in the tree will always depend on the attribute dimension--there is no "independent" branch for the attribute dimension. These conventions will be adhered to throughout the remainder of this document. We will provide additional motivation for these conventions shortly.

Although the $n$-dimensional cube is an intuitively appealing way of structuring data, it is clearly not storage-efficient nor does it take advantage of the natural structure of the model. To illustrate, assume that variable 4 in Figure II.A.4. is National GNP and that the 


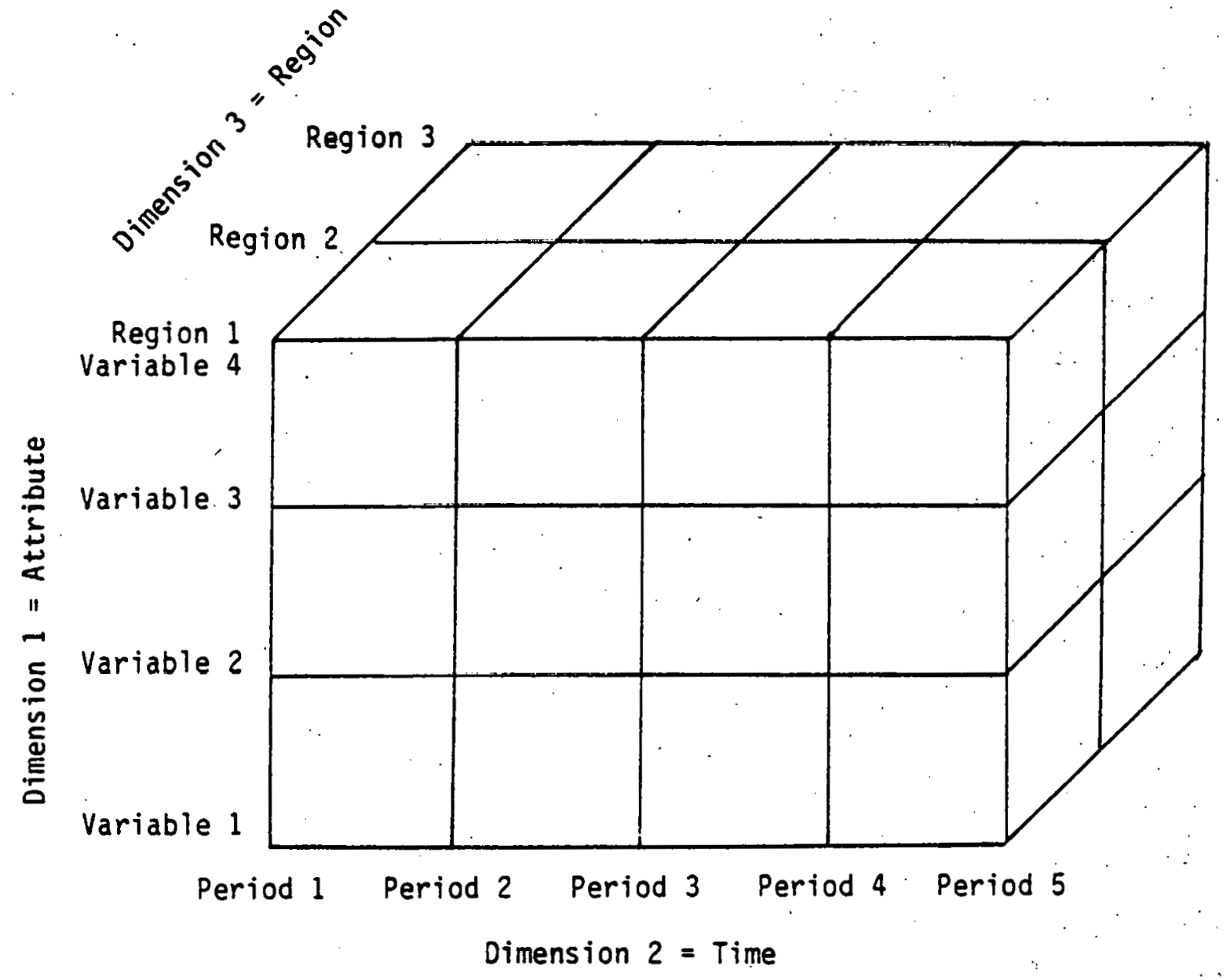

Figure II.A.4 THREE DIMENSIONAL CUBE 
'three regions are the Eastern, Central, and Western states. Then, since, National GNP does not vary by region we know that the value stored at location $(4, i, j)$ for a given $i$ is the same for all $j(i$ is the time index and $j$ the region index). Thus, in order to use this cube directly, one would have to store a good deal of redundant data or, alternately, define a number of unused storage locations. Comparing this three dimensional case to the two dimensional case above, we can see that the amount of redundant data can grow dramatically as the -number of dimensions increases. If our data base management system is to handle large as well as small models, we cannot hope to retain the n-dimensional cube as our fundamental data structure.

The generalized tree for the three-dimensional data structure is shown in Figure II.A.5. This tree is depicted as a decision tree to emphasize that it is a decision on the part of the modeler as to which data elements will be dependent upon which dimensions. Notice that all the variables are dependent on the first dimension-- the attribute dimension. This is consistent with the convention developed above. In Figure II:A.5 suppose that we index each of the "Dependent on Dimension $i$ " branches by a 1 and each of the "Independent of Dimension $j$ " branches by a 0 for $i=2,3$. (Since the branches at the attribute. level are always dependent, we do not need to index them.) Each terminal branch of the tree, can then be indexed by a 2-vector of I's and $0^{\prime} s$. If we view this 2-vector as a two digit, base 2 (binary) number, we will have attached a unique index from $0-3$ to each of the terminal branches. Thus we will have associated a unique integer index with each possible combination of dependent and independent dimensions. Note that each terminal branch of 


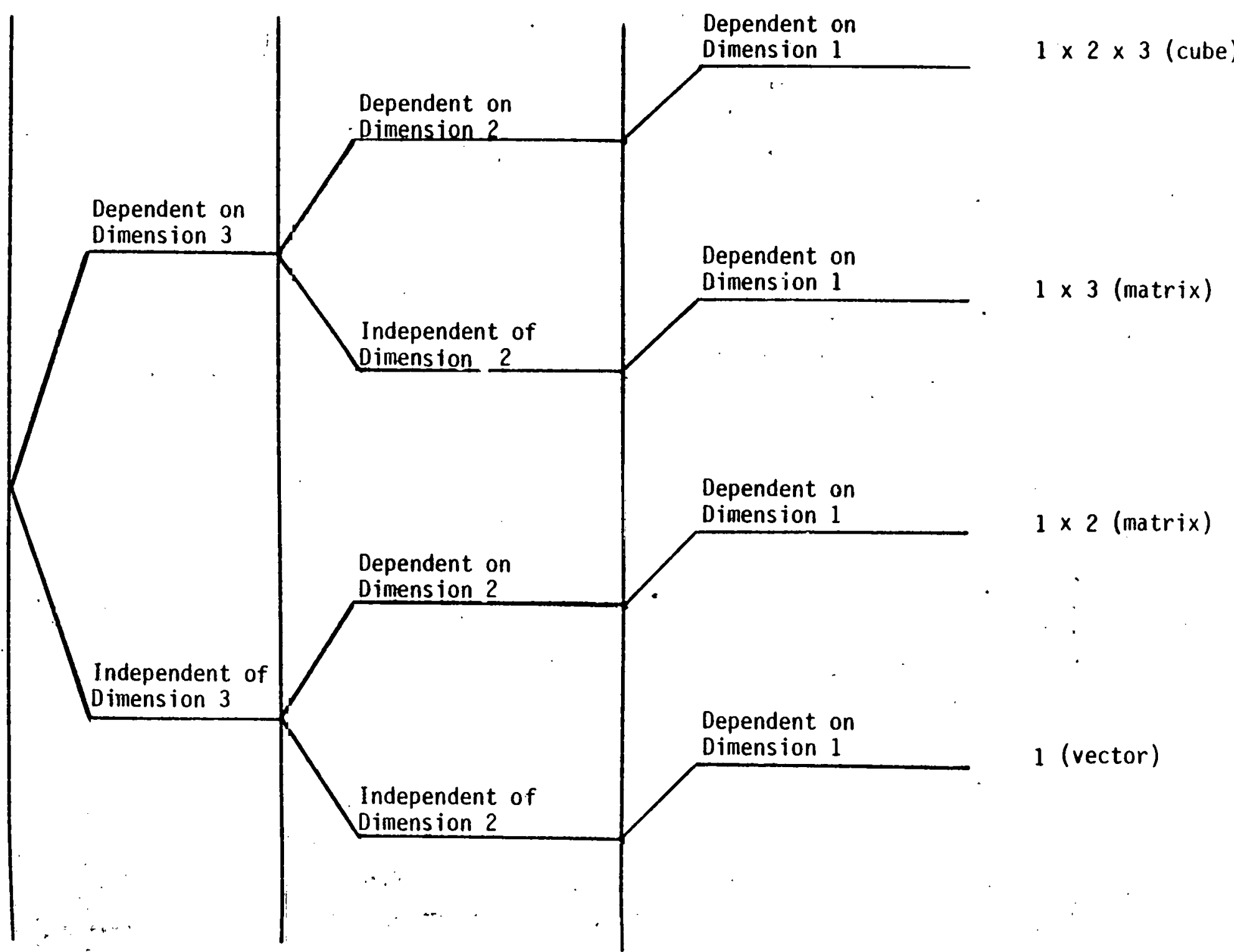

Figure I1.A.5: DEPENDENCE DECISION TREE 
this tree may actually correspond to a set of data values. We define a data set as the collection of all data values indexed by the same binary vector, i.e., the set of all data which are dependent upon the same combination of dimensions.

Returning to our earlier present value example, we can redraw the tree shown in Figure II.A.2 in binary tree form as shown in Figure II.A.6. We have indexed all the time varying branches with a 1 and combined them into a single branch in the binary tree, and similarly for the time independent branches. The data contained in this binary tree is categorized as follows: those variables which are dependent on time $\left(c f_{i}\right)$ and those which are independent of time $(P V, N, r)$. Thus, since $P V, N$, and $r$ have the same dimensional structure, they are in the same set. All the $\mathrm{cf}_{i}$ 's lie in a different set.

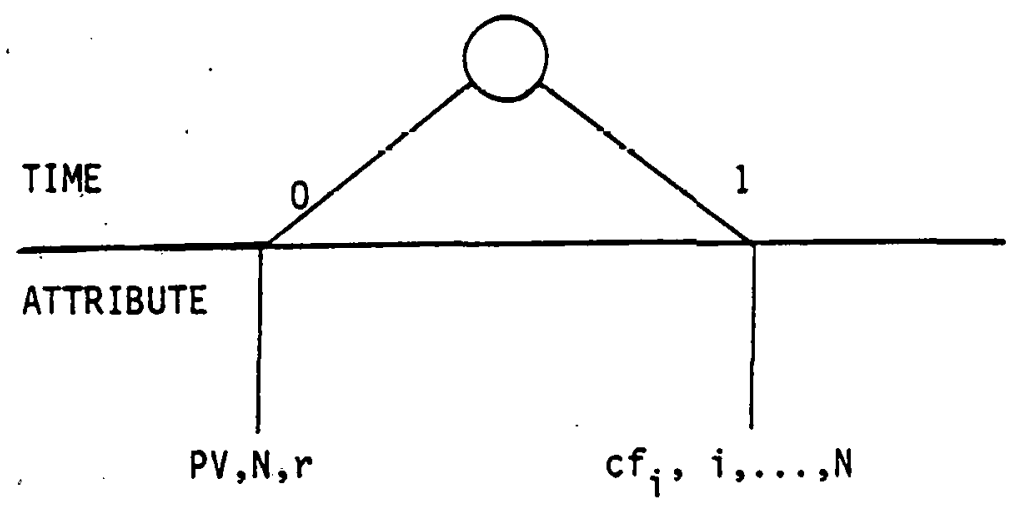

Figure II.A.6. BINARY REPRESENTATION OF A TREE

Note that our use of the term "data set" is different from the usual IEM definition. 
All the data in a hierarchical data tree can be categorized into distinct data sets by this method. This concept of a data set is fundamental to the design of both the Data Transfer System and the Executive System which will be described in the next sections.

One of the primary motivations of model building: is to decompose large systems into one or more smaller subsystems, to build models of these subsystems, and to integrate them back into the larger system model. The fundamental input data structures tend to be simple because they are specific to small subsystem models. In practice, then, we consistently find that very few elements of input data depend simultaneously on more than two or three dimensions. If a data cube representation such as that in Figure II.A.4 were used for such a model, it would be comprised almost entirely of redundant values or unused positions. Expressed in terms of the decision tree in Figure II.A.5, the modeler usually chooses many of the "Independent of Dimension $i$ " alternatives in designing his subsystem models. However, by combining his system of equations in appropriate ways, he builds up complex multi-dimensional dependencies in the output data from a set of simple one, two, or perhaps threedimensional dependencies in the input data. Hence, while the output data might require a more complex dimensional treatment, the input data rarely does. Our hierarchical tree method of data management allows great flexibility to use whatever set of dimensions is required either at the set level or the entire model data base level. 
B. 'THE EXECUTIVE SYSTEM

The Executive System contains a set of routines that allow the user to interactively display, insert, delete, and change values in the hierarchical data tree. In fact, the Executive is used to:

1. Define the structure of the hierarchical tree, and

2. Attach data to particular nodes in the hierarchical tree. The Executive is designed to interact directly with the hierarchical tree using what we call hierarchical tables which are subsets of data sets. A data set is an n-dimensional cube that contains all the data which are dependent on those $n$ dimensions. A nierarchical table is an $m-c i-$ mentional subset of the $n$-dimensional data set $(m \leq n)$. Using the Executive, the user constructs hierarchical tables which collectively contain all the data needed for his application and fills those tables with numerical values. Figure II.B.I illustrates at a general level the function of the Executive.

While hierarchical tables will contain a subset of the number of dimensions used in the model, the philosophy underlying the Executive is that the user can only work with, at most, a two-dimensional table at any one time. Thus the Executive at any given moment, works with only a two-dimensional "slice" of an m-dimensional hierarchical table. The Executive allows the user to modify these m-dimensional tables by sequentially modifying two-dimensional slices until the entire table has been spanned.

We will now illustrate the basic concepts underlying the Executive using a simple example. We will show how to make the transformation from a data set to hierarchical tables and finally to the hierarchical 

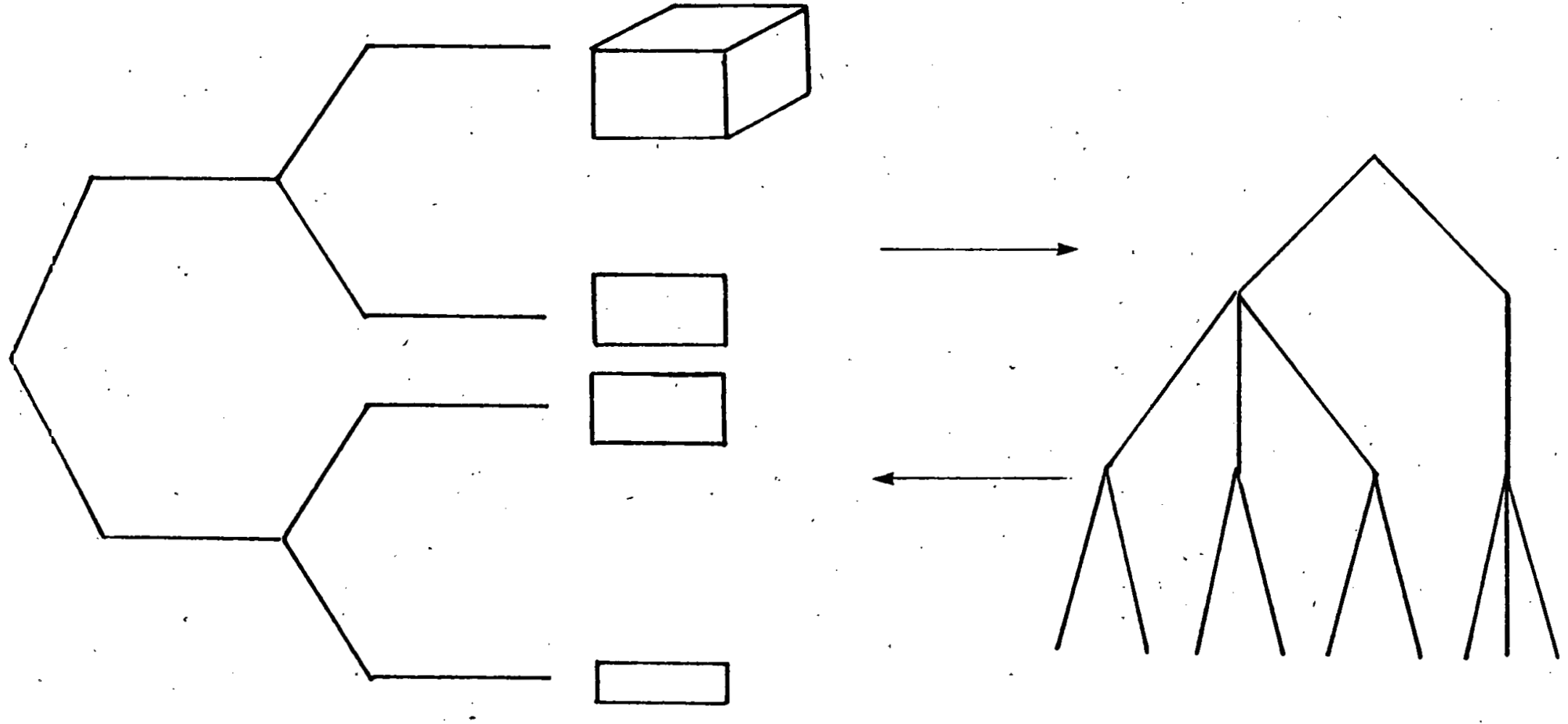

Figure II.B.1 THE EXECUTIVE SYSTEM 
data tree. The example is shown in Figure II.B.2 where we begin with the two tables on the left. Creating the first two-dimensional slice in the figure and placing. it in the data base creates the lefthand part of the tree (that part for which the value of dimension 3 is 1 ) and puts the data values in the appropriate positions at the bottom of the tree. The second two-dimensional slice and the right hand part of the tree are similarly related.

For this example all the data depends on all three dimensions -(all values are in the same data set). Thus a single three-dimensional table would be sufficient to enter or display the data. However, by fixing dimension 3 at each of its two values the user can work with two two-dimensional slices--a much more tractable task. If we instead fixed dimension 2, three two-dimensional slices would be required; fixing dimension 1 would require four two-dimensional slices. In addition the user can specify which dimension he wishes to vary across the rows and which to vary across the columns in case he wishes to transpose the tables in Figure II.B.2. Thus in defining the tables, the user has considerable flexibility in deciding which two-dimensional slices to work with. He should choose the aiternative that presents the most logical display format for his needs. The two other alternatives for the current example are shown in Figure II.8.3.

It is essential to note that there is a unique mapping from a set of hierarchical tables to a hierarchical data tree. The purpose of the Executive is to exploit this mapping so that the user need not be burdened with its details when he wishes to change his model or to change data values in his mocel. 


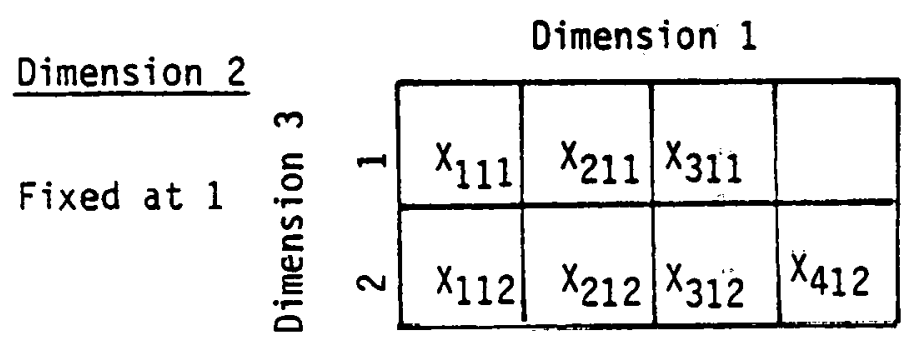

Fixed at 1

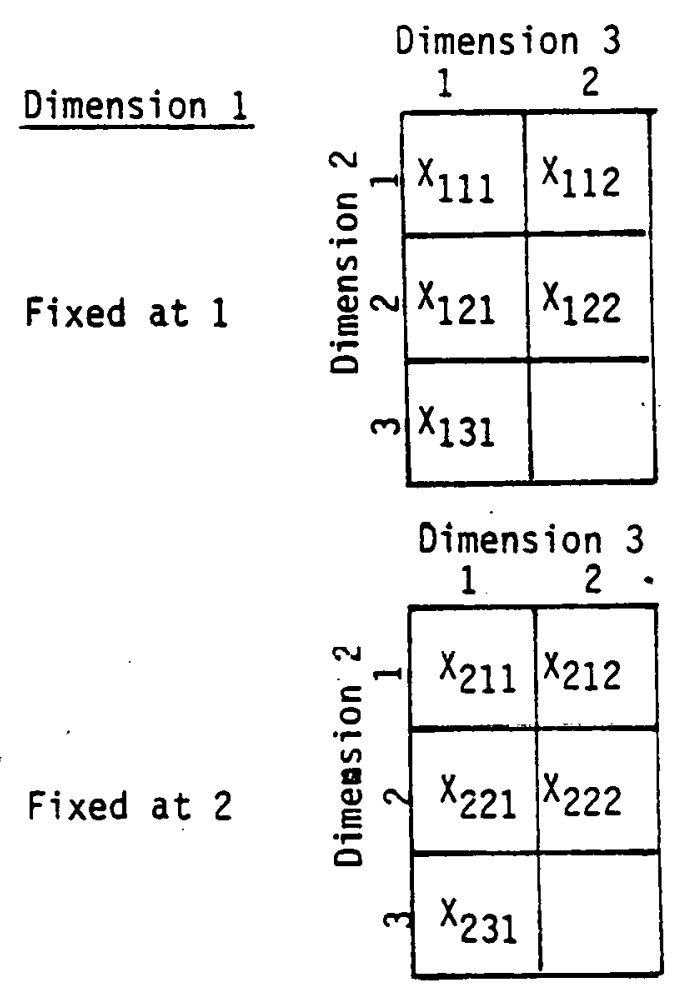

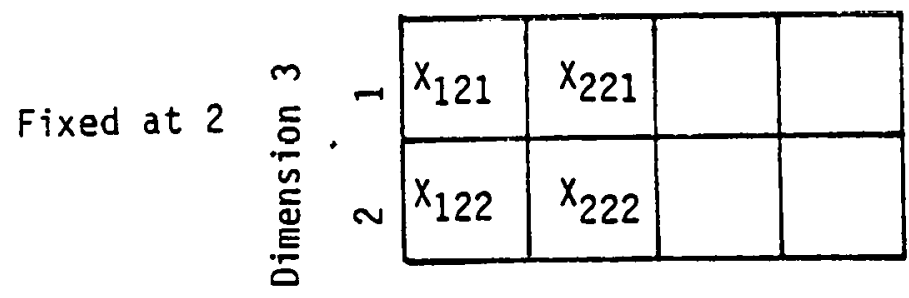

Fixed at 3

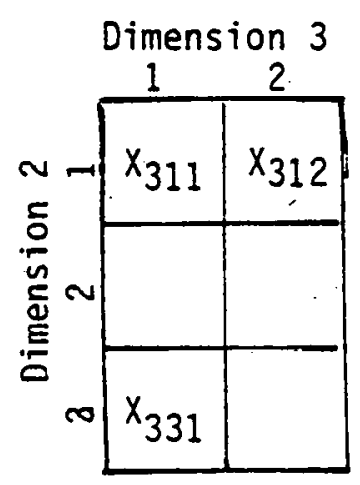

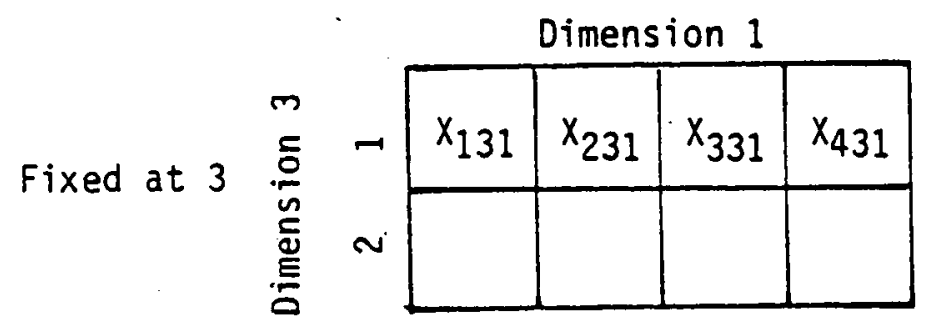

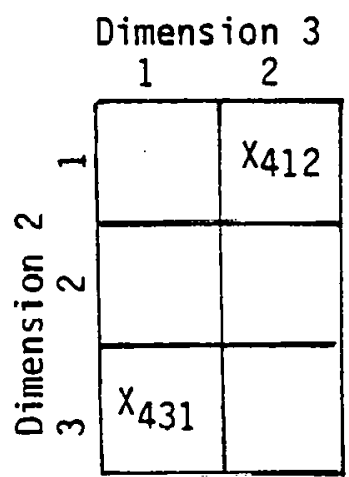

Figure II.B.3 ALTERNATIVE TABLE DEFI.VITIONS 
Now let us return to the PV example of the previous section. In the simplest case the interesc rate is constant, so there are three parameters that are independent of time: $N, r$, and PV. These parameters are all in the same data set, and may be placed in the same table. The cash flows, cfi, depend on time and must be placed in a different table. The two hierarchical tables for this example are shown in Figure II.B.4. Each of these tables, including data elements, row names, column names, titles and footnotes can be entered with five Executive System instructions. The actual Executive commands are described in Section IV.A.3. (Note that although PV will be calculated by the model, the user must specify a position in the tree to hold the value of PV. He does so by placing an arbitrary number in the PV position of the left hand table in the figure.)

Extending our simple example, suppose we wish to evaluate $M$ cash flows, each of which covers the same number of time periods and uses the same interest rate. This would require the introduction of a new dimension, which we might call plant; each cash flow would correspond to a different plant. We would then have three data sets and could conveniently enter or display the entire data base using three hierarchical tables. One table would contain $N$ and $r$, which vary neither by time nor.plant; one table would contain $P V_{1}, \ldots, P V_{M}$, which vary by plant but not by time; the other table would contain the cash flows, which vary by plant and by time. The hierarchical tree and one way of structuring the hierarchical tables are shown in Figure II.B.5. The tree in the figure follows our branch numbering conventions, adding "plant" to the list of dimensions. 


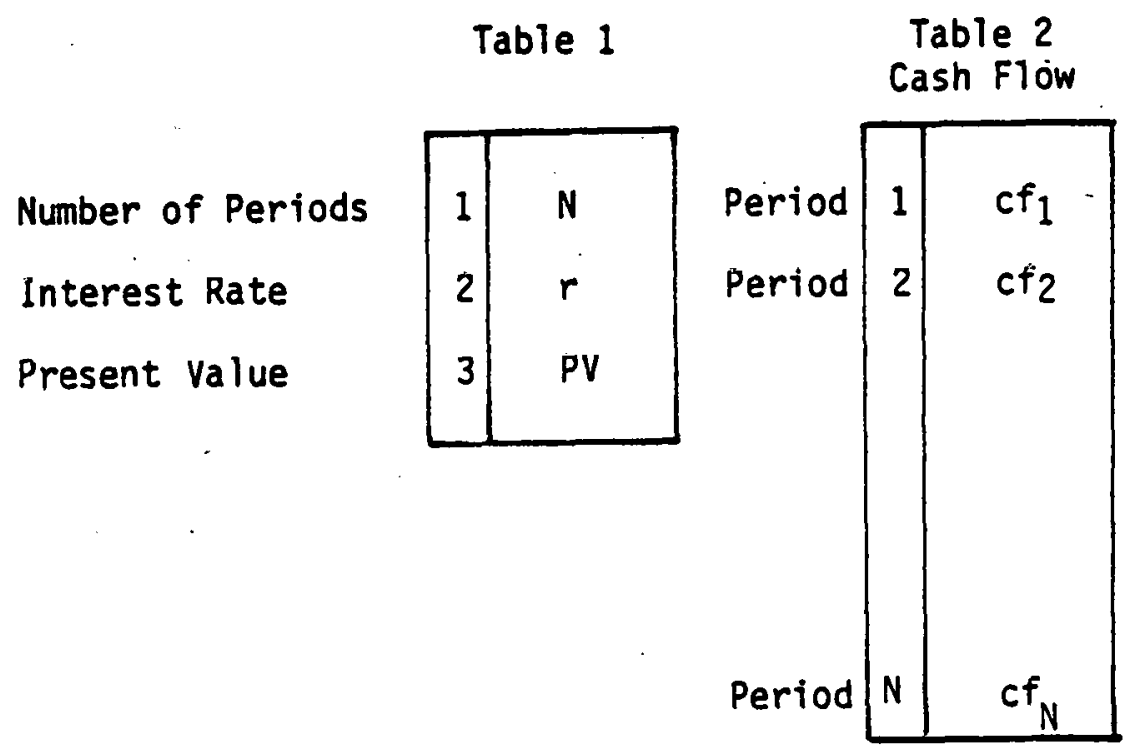

Figure II.8.4 TABLES FOR PV EXAMPLE 

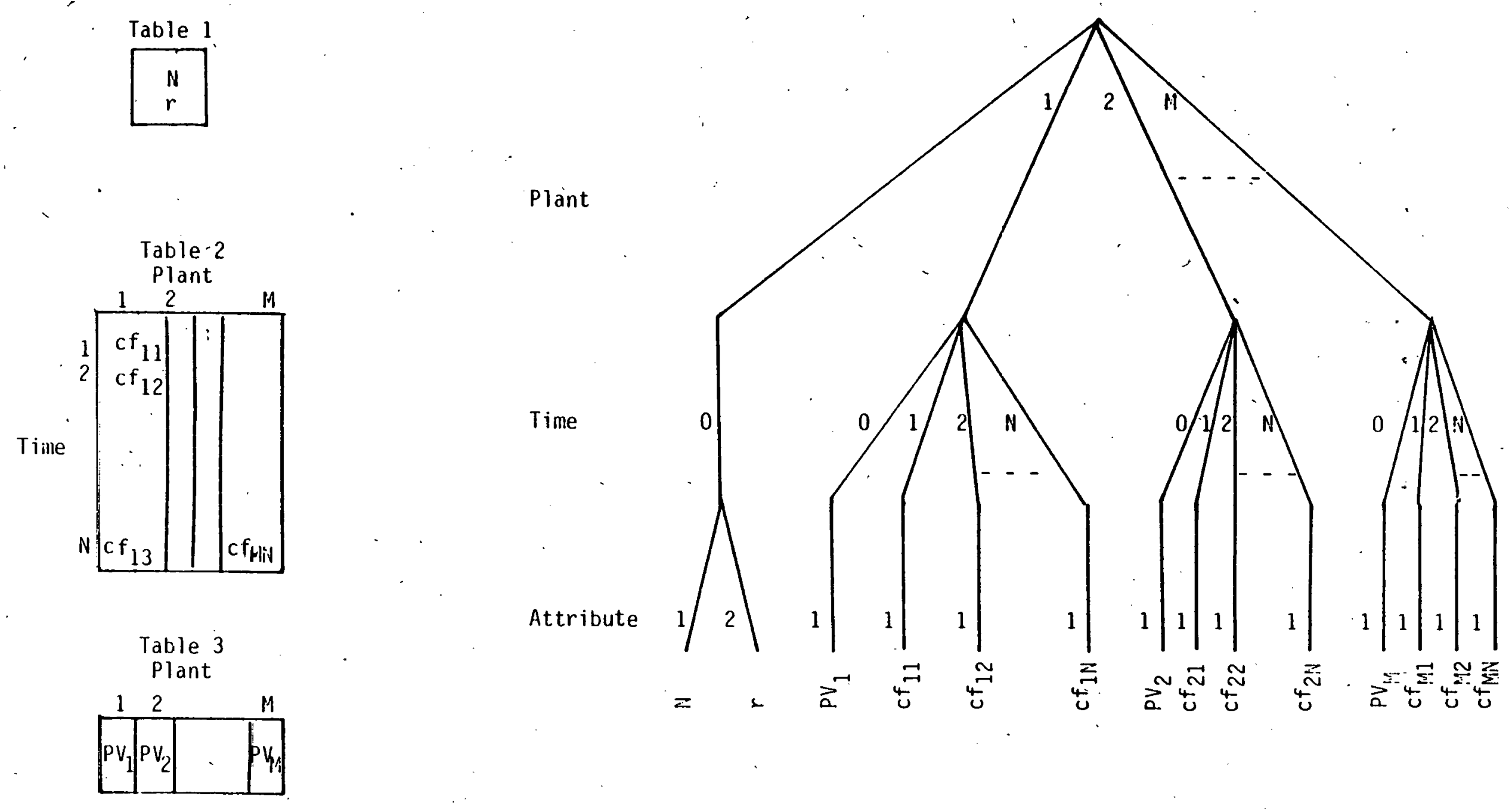

Figure 11.B.5, EXPANSIOH OF. PV EXAMPLE 
A description of the operation of the Executive is given in Chapter V. Its use is described in Chapter IV.A. 


\section{THE DATA TRANSFER SYSTEM}

Given the existence of a hierarchical data base inferred from the dimensional structure of the model, the function of the data transfer system is to control the flow of data between the model logic and the hierarchical tree. As currently implemented, this requires four distinct steps. We will describe these four steps in some detail as insight into the operation of the data transfer system is crucial in understanding how the data base management system interfaces with the model logic. The four steps executed by the data transfer system are illustrated graphically in Figure II. C.I.

Step 1. The first step begins with the dimensional representation (i.e., hierarchical tree) described in Sections $A$ and $B$ above. The data transfer system logic contains an algorithm that in effect generates a digital representation of this tree structure. The algorithm works by visiting each branch of the tree in a particular order. In so doing, it keeps track of the structure' of the tree so that when it encounters a value (i.e., a terminal branch), it can place that value in the proper position in a single vector known as the $\underline{0-V e c t o r}$. When the tree has been completely traversed, all the data necessary to run the model and report its results have been placed into the D-vector. In addition, the data transfer routine will have created a matrix of pointers that gives the location of each piece of data within the D-vector. 

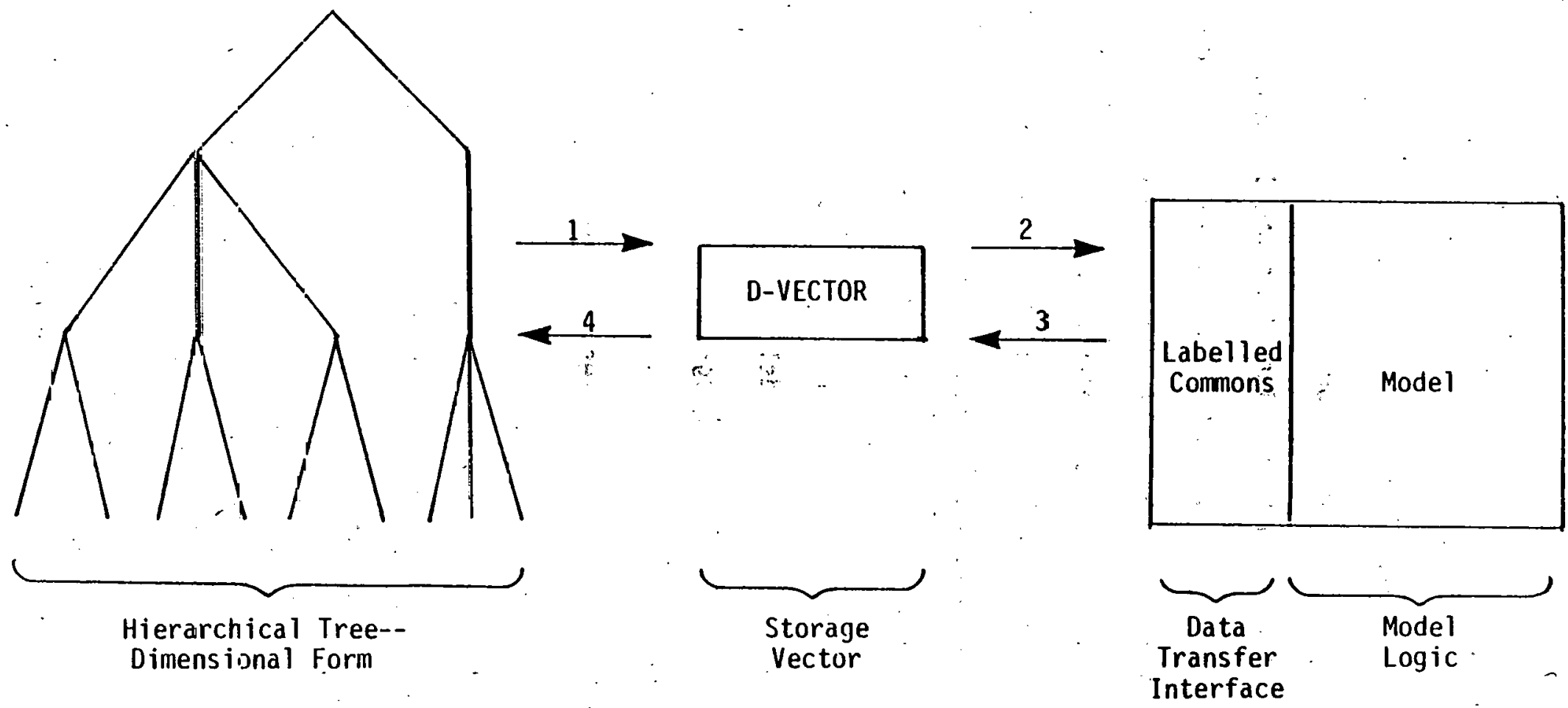

Steps: 1. Data (input) collapsed from tree into D-vector

2. Data (input) moved from D-vector into model

3. Data (results) moved 'from model into D-vector

4. Data (results) moved from.D-vector into tree

Figure II. C.I DATA TRANSFER OPERATIJN 
Step 2. Once the D-vector has been constructed and the indexing scheme implicit in the hierarchical tree structure set up, we can readily retrieve subsets of data from the 0 -vector and transfer them to the model logic in the form the model logic requires. This is accomplished by placing the appropriate values from the D-vector into "labelled common" areas. After this operation, each labelled common area contains the data values needed by the model in a'configuration known to the model. The model accesses these values by simply "attaching" its own internal variable names to these values, thus avoiding the need for cumbersome and inefficient FORTRAN READ and FORMAT statements to be placed into the model logic itself.

This transfer of data from the D-vector to the model is actually performed as the model runs. As the model processes.a particular subroutine, the data transfer program accesses the data necessary for that subroutine. When the model processes a different subroutine, the data transfer program accesses the data necessary for the new subroutine. This featire is crucial in building itarative network models.

The structure of the labelled commons has the appealing property that the model builder need not rename or re-index his internal variables to be consistent with the D-vector or any other set of conventions. The labelled common interface allows the modeler to retain his code intact and to pass in data under any naming or indexing conventions he wishes. The only user requirement is that he make these conventions explicit and categorize his internal variables along dimensional lines. 
In this procedure steps 1,2 , and 4 require access to data residing in the D-vector. Each access requires a single subroutine call in the computer implementation. Note that since $N, r$, and $c f_{j}$ are not changed within the model (they are input data), they need not be replaced in the D-vector base after the model is run.

Although the present value example is quite simple, it contains many of the important components of data transfer to be found in larger models. It is important to notice that the labelled common definitions used represent only one of several equally valid possibilities. The data transfer system allows the modeler great flexibility in specifying which data to transfer into which labelled common areas and hence into which variable names in the user's program.

Step 3. This is the reverse of Step 2 -- intermediate and final results from the model are transferred back to the D-vector. The algorithm used here is the exact inverse of the one used in Step 2 and was alluded to in the simple procedure outlined above. Just as with the transfer of data into a particular model subroutine, the transfer of results back out to the $D$-vector is performed as the model runs.

Step 4. When the model solution is complete, it is convenient to reconstruct the hierarchical tree and to place the values from the D-vector on the appropriate terminal branches. Once the model has run, many of these values will be model results, and, of course, many will still be input data. When this reconstruction process is complete, we will have a full dimensional representation of both the model input data and results, all residing in a single hierarchical data base. Furthermore, because of its dimensional structure, this data is easy to display in a meaningful form using the Executive System. 
The transfer program is designed to map either an entire data set (in the above defined sense), or a subset of a data set into a single labelled common area. This approach is based on the observation that the model will usually need to access most of the values in the same data set (i.e., most of the dimensionally similar values) at the same time. Of course, the model can access several data sets simultaneously by simply using several labelled comnon areas simultaneousiy.

To see how this principle is used in data transfer, consider again the one-equation present value model discussed previously. Suppose the user defined the following labelled common areas:

Labelled Common \#

1.

2.

3.
Variables in Labelled Common

i. $N, r$

PV

cf $_{i} \quad i=1, \ldots N$

Note that these labelled common definitions follow the above principle: subsets of set 0 in Figure II.A.6 (independent of time) are mapped into labelled commons one and two while all of set 1 in Figure II.A.6 (dependent on time) is mapped into labelled common three.

With these labelled common definitions, an outline of a routine to compute PV using the data transfer system is given below:

1. Fill labelled common 1 with values of, $N$ and $r$ by accessing the $D$-vector.

2. Fill labelled common 3 with values of $\mathrm{cf}_{i}, i=1, \ldots \mathrm{N}$, by accessing the D-vector.

3. Compute PV in the model.

4. Store labelled common 2, thus storing PV in the D-vector. 
A guide to using the data transfer system is given in Chapter IV.B. Chapter VI describes the algorithms that are used in the data transfer system. 


\section{SYSTEM DESIGN}

The computer modeling software can be regarded as the combination of the Executive System and the Data Transfer System as shown in Figure II.0.1.

Figure II.0.2 shows the conceptual relationship of the various physical components of the DFI Computer Modeling Software. The Data Base Storage System (DBSS) contains the data for several model runs. The Data Transfer System (DTS) takes the data for a specific run and places it in the labelled common area of the model. While the model runs, model output is placed into the Data Base Storage System. The Executive allows the user to draw this output data from the DBSS in table format. He can then edit input tables and replace them or display results tables.

This system design allows the user considerable flexibility in structuring both his logic and his data. The Executive allows him to both enter and display his data in a clear and convenient fashion. The Data Transfer System allows him to input and output data in the fashion most convenient for a specific model. The user's interface with the model logic is simplified since data input and output is no longer a difficult problem.

A flow chart for the complete system is shown in Figure II.D.3 Arrows represent directions of data flows, cylinders represent storage, and rectangles represent programs. Dotted arrows denote user option.

As can be seen in Figure II.D.3 the Executive allows the user the options of displaying his tables on a CRT, printing them offline, or printing them on a teleterm. The offline option is convenient when large numbers of tables need to be printed. 


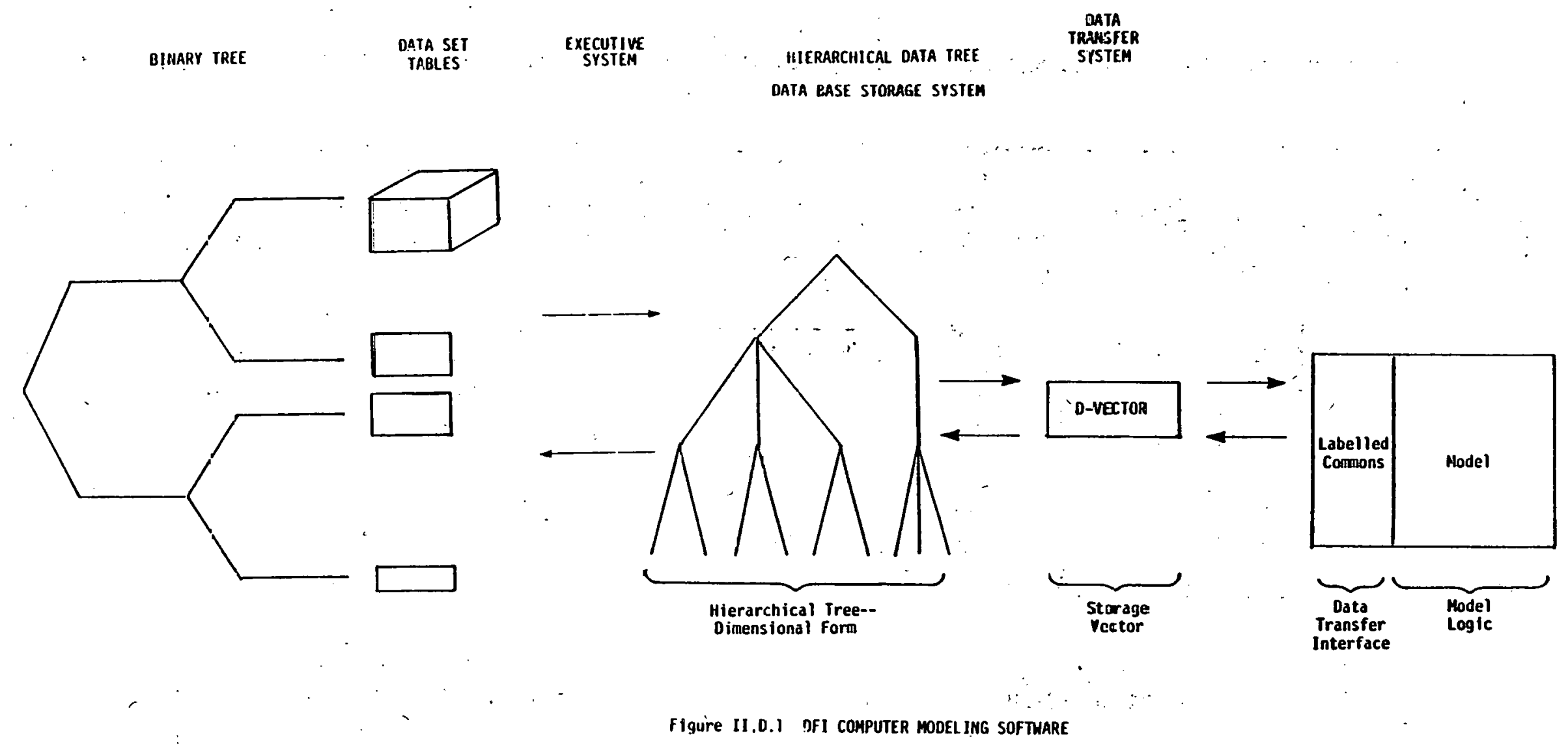

Figure [I.D.] DFI COMPUTER MODEL ING SOFTMARE 


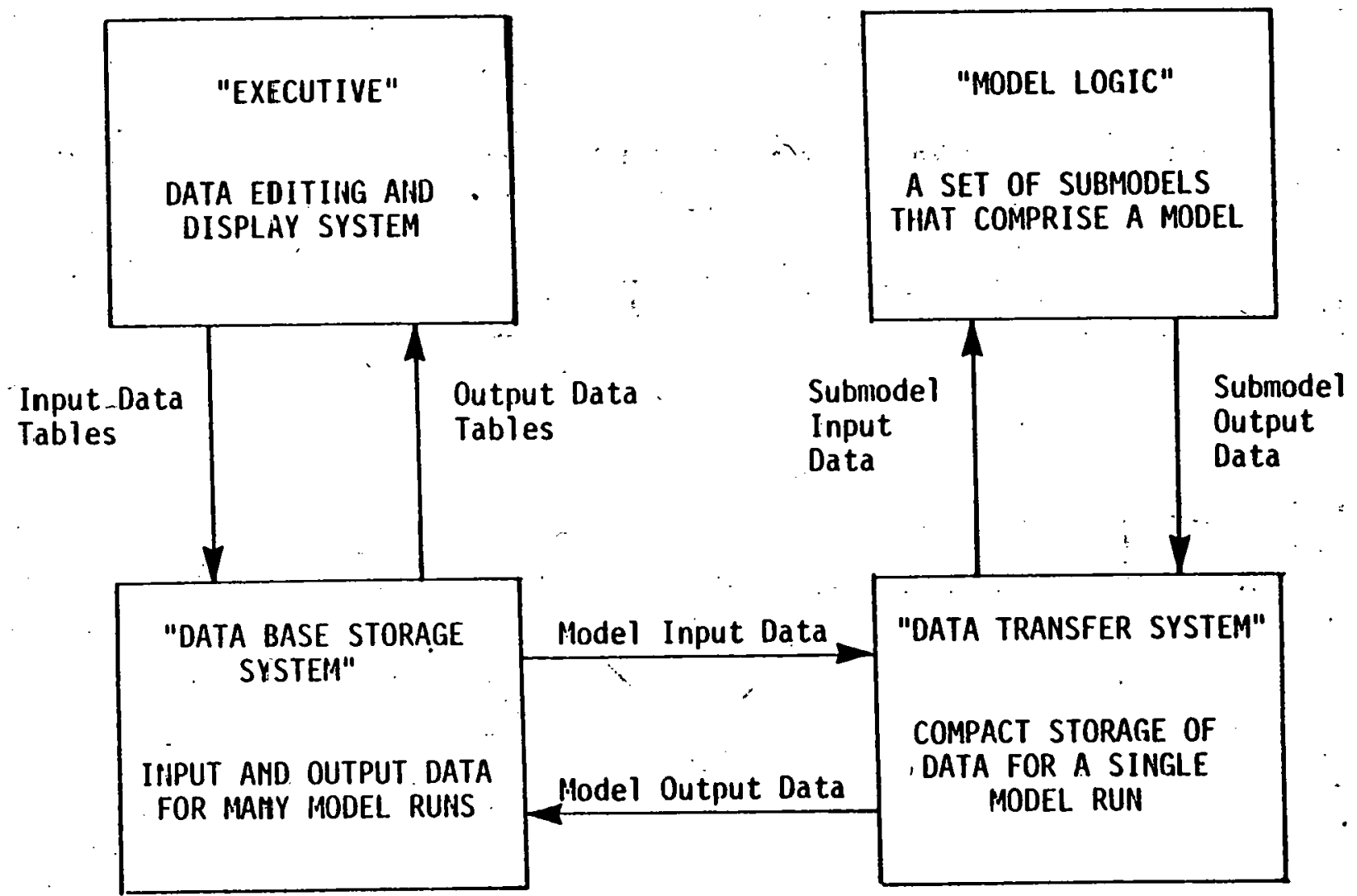

Figure II. D.2 COMPONENTS OF THE DFI-COMPUTER MODELING SOFTWARE, 


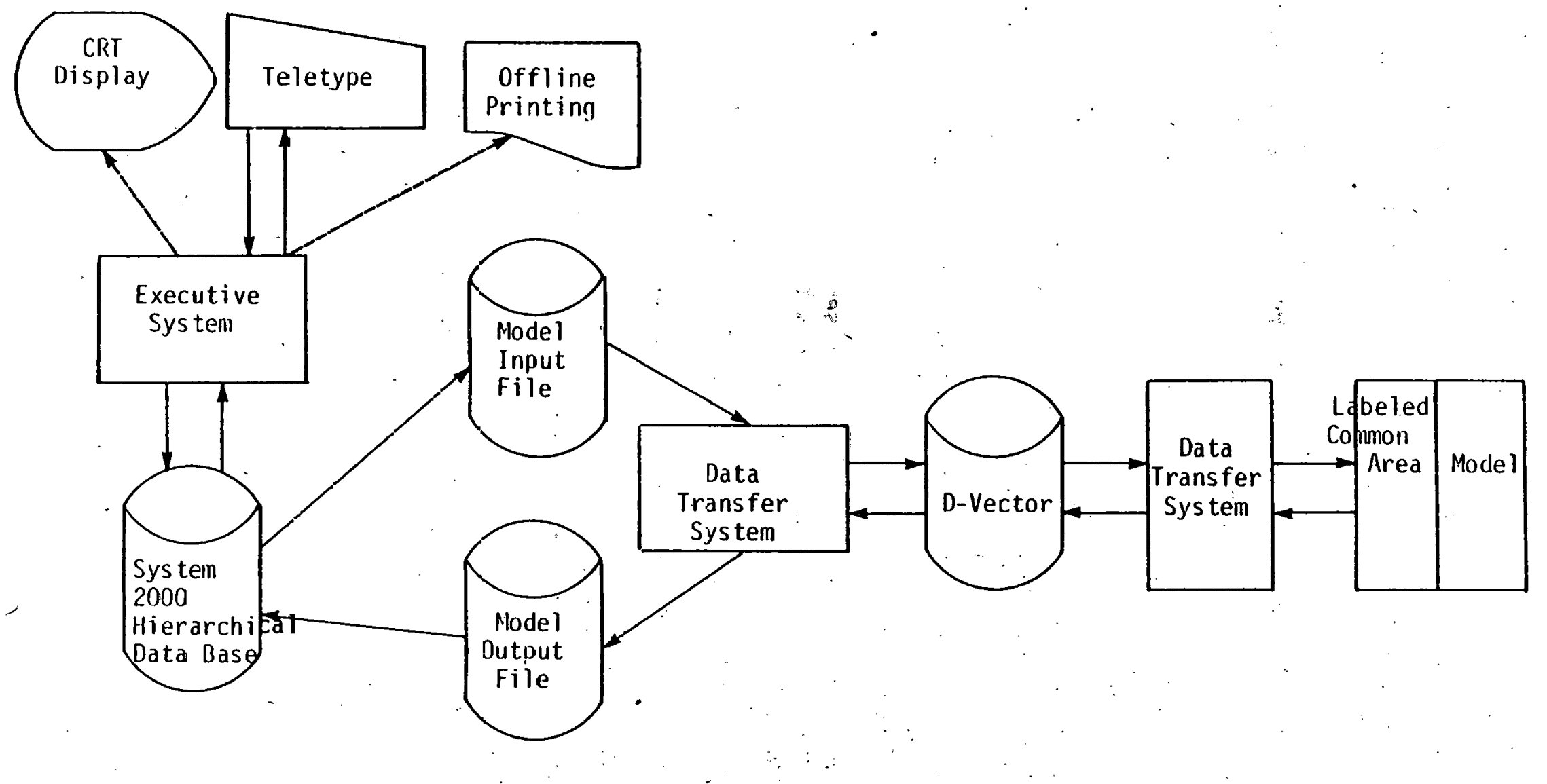

Figure II.D. 3 DF I COHPUTER MODELING SYSTEM FLOWCHART 
The only permanent storage in the whole system is the System 2000 Hierarchical Data Base. The input file, output file, and D-Vector are all temporary and may be purged after the model has been run.

The user actually has three options in running the Data Transfer System, these three arising from the observation that the D-vector itself can be stored directly rather than having to be read back out to the hierarchical tree:

1. Read in data from a hierarchically structured input file. Read out data to a hierarchically structured output file.

2. Read in data from a hierarchically structured input file. Read out data to a binary representation of the D-vector.

3. Read in data from a binary representation of the D-vector. Read out data to a binary representation of the D-vector

In general, the user will run the model according to option 1, since it is the only one which allows the model results to be displayed using the Executive. The other two options have been provided to allow an interactive debugging capability which is more fully explained in Section IV.B. 
CHAPTER III

METHODOLOGY OF THE COMPUTER MODELING SOFTWARE

\section{†. GENERAL PROPERTIES OF TREES}

In order to motivate the use of tree structures to represent mode] data bases, we will discuss some of the general properties of trees that make them particularly suitable for our application. We will discuss these general properties in the context of the simple example in Figure III. A. I.

We begin by noting that the tree is a multi-level, hierarchical structure consisting of branches and nodes. Each branch in the tree has a node at both ends. If we think of the nodes as distinct positions in space, the tree represents an orderly structure for specifying where each of those positions lies relative to the starting position or root (and hence relative to each other). In fact, the position of each node in the tree is uniquely specified by the branches above it.

Suppose now we have a number of distinct sets of information and we wish to "catalog" them so that they can be uniquely accessed. Viewing the tree structure as a mechanism for distinguishing among nodes, suppose we associate each of the information sets with a different node in the tree. By so doing, we can use the "node distinction" property of the tree to distinguish among our information sets. Furthermore because the tree contains a "road map" where each of these nodes is located, the tree itself can be viewed as no more than an indexing mechanism for the information sets. Hence in our application, each node in our hierarchical tree will serve as a repository of information 


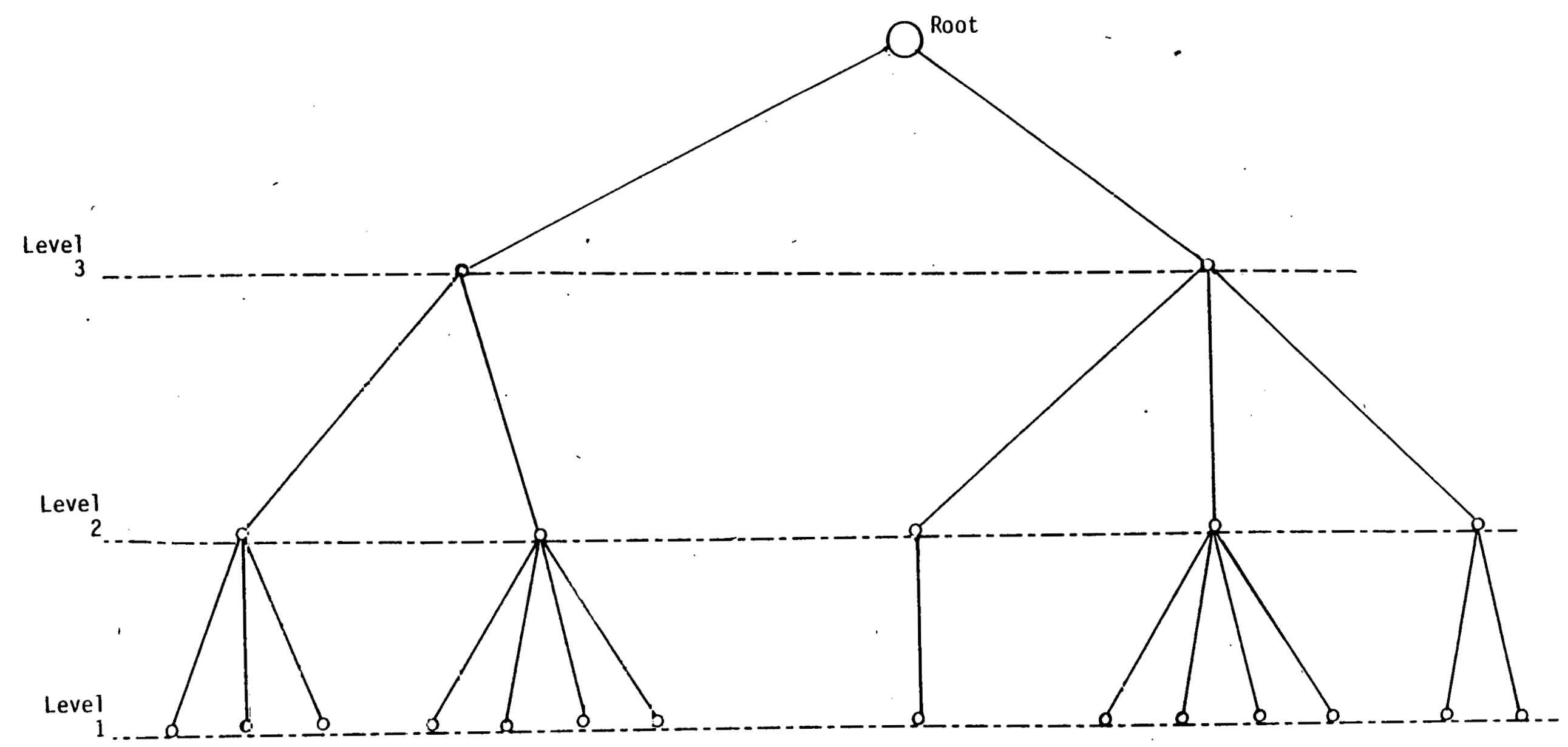

Figure III.A.1: EXAMPLE TREE 
which is uniqueily indexed by the branches above that node. To illustrate this interpretation of nodes in the tree, we have constructed Figure III A.2.

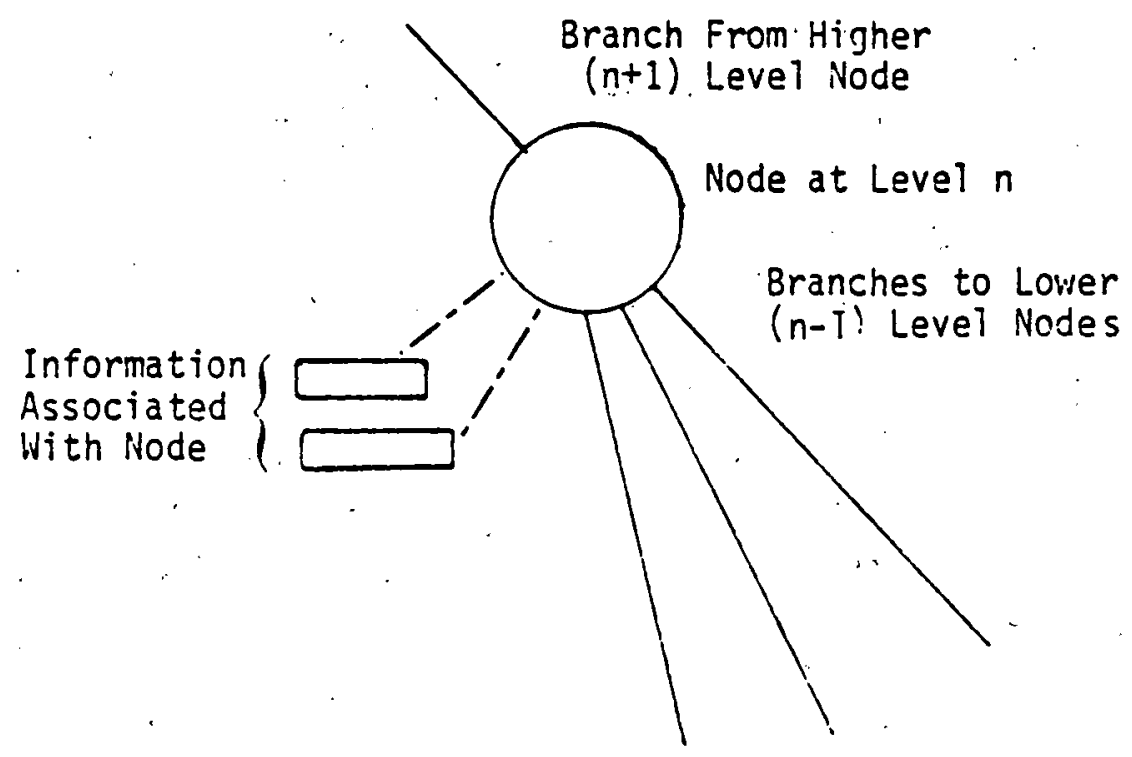

Figure III.A.2: NODE IN A HIERARCHICAL TREE

\section{B. STANDARD TREE STRUCTURE}

Both the Executive and Data Transfer System make several assumptions concerning the structure of the hịerarchical data tree. Taken together, these assumptions constitute what we will call Standard Free Structure. Any firerachịcal data tree that is to be interfaced with the DFI computer modeling software must necessarilysatisfy the assumptions of standard tree structure. Since both the Executive and the Data Transfer system preserve standard tree structure in their operation, the average user 
does not need to be concerned with these principles. The topic is crucial, however, to anyone seeking to understand the details of operation of the computer modeling software.

In order to introduce this topic, we have redrawn the hierarchical tree that appeared in Figure III.A.I in standard tree form: it appears. in Figure III.B.1 We will discuss the conventions underlying the transition between the two figures and will motivate the assumptions underlying the standard tree structure.

1. Branch and Node Naming/Numbering Conventions

As illustrated in the tree in Figure III.B.I the levels in the tree correspond to the dimensions of the data base. We have adopted the convention that the dimensions will be indexed from the bottom (i.e. the lowest level) to the top (i.e. the highest level) in the tree. 1 Furthermore, dimension 1 will always be termed the attribute dimension and will be used to count the number of nodes at level 1 given a particular setting of dimensions $2, \ldots, n_{D I M}$. This distinction at level 1 arises from the convention that all data values are explicitiy dependent on the attribute dimension, i.e. on dimension 1 in the tree. The CMS further assumes that a node at the attribute level never contains both an integer and a real value. An attribute-level node can contain a real value, an integer value, or neither one, but not both. Thus, at most. nne data value is assuciatcd with each attribute node in the tree. (When we refer to a data value, we are referring to numerical data.)

1. The highest level dimension will be denoted noIM in this chapter. 


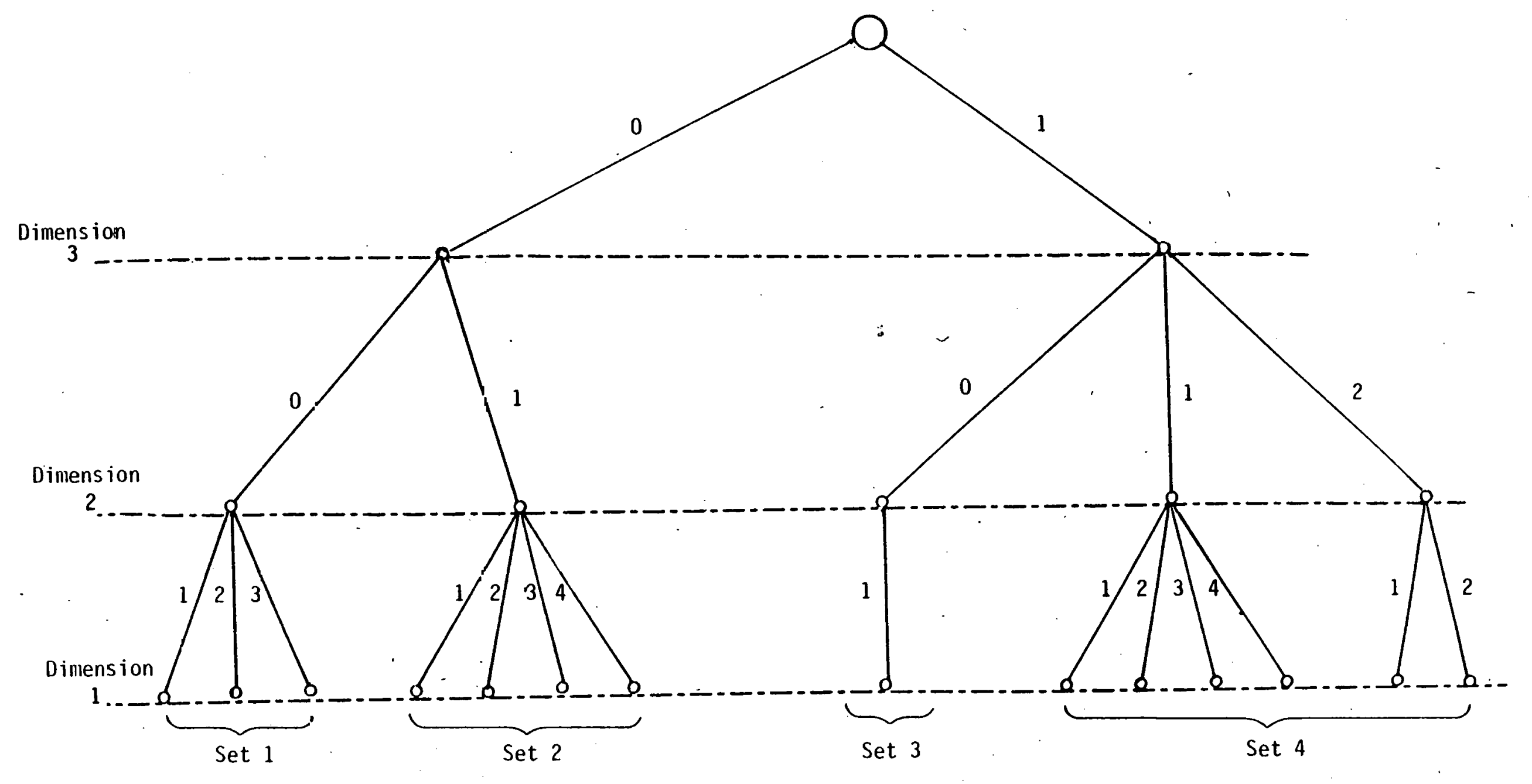

Figure III.B.1: "EXAMPLE TREE IN STANDARD TREE FORM 
Figure III. B.1 also illustrates the numbering convention for descendent branches and nodes. At level 2 (i.e. dimension 2) the descendent branches are always indexed beginning with the index 1 . Expressed alternatively, the number of nodes at level 1 are indexed beginning with 1. Figure III. B. 2 illustrates. At levels $j=3,4, \ldots, n_{\text {DIM }}$, the indexing scheme distinguishes between the "independent of dimension

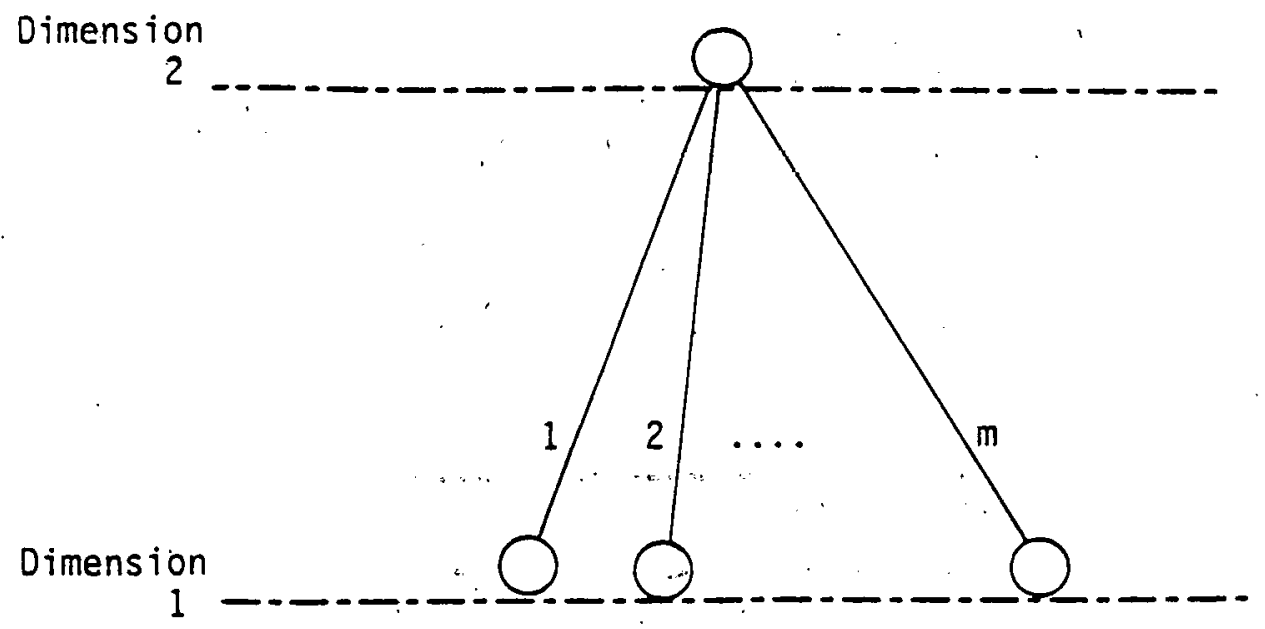

li.

Figure III.B.2: NUMBERING CONVENTION FOR DESCENDENT BRANCHES AT LEVEL 2

$j-1 "$ and the "dependent on dimension $j-1 "$ cases. Since there is only one branch corresponding to the "independent of dimension $j-1$ ". case, it is indexed with a zero. The "dependent on dimension $j-1$ " branches are indexed sequentially beginning with 1. Figure III, B.3 illustrates. The reader will note that these indexing assumptions have been used in the hierarchical tree in Figure III.B.1. Finally there are no descendent branches at dimension 1 in the tree -- they are the terminal nodes 


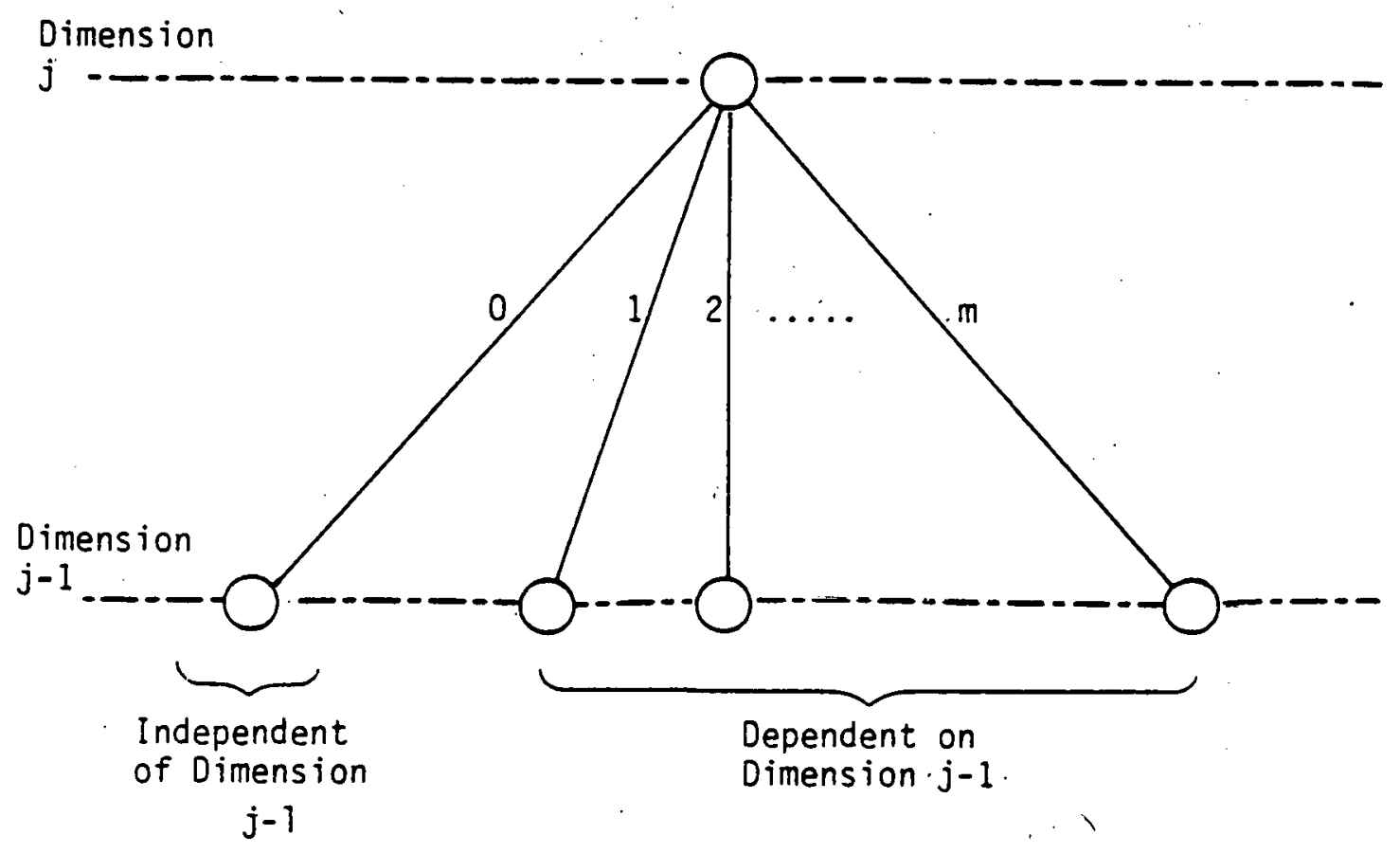

Figure III.B. 3 NUMBERING CONVENTIONS FOR DESCENDENT BRANCHES OF LEVEL $j=3$ OR ABOVE 
in the tree and contain only data. Because these branch indexing conventions are central to understanding the transfer system, we will summarize them below:

1. Nodes at dimension 1 have no descendents.

2. Nodes at dimension 2 have their descendent branches numbered from left to right starting with 1 .

3. Nodes at dimension 3 or higher have their. descendent branches numbered from left to right starting with 0 .

Nodes in a hierarchical tree are customarily referred to using "familial". terminology as illustrated in Figure III.B.4. For node $x$ at dimension $n$ in the hierarchy, the node at level $n+1$ which shares a branch with $x$ is referred to as the parent of $x$. Nodes which share the same parent are siblings. A sibling of node $x$ which is on a lessernumbered branch than (i.e. to the left of) node $x$ is referred to as an elder sibling while one on a higher-numbered branch (i.e. to the right of) node $x$ is a younger sibling. For node $x$ at dimension $n$, each node at level $n-1$ sharing a branch with $x$ is referred to as a descendent of $x$.

Perhaps the most important requirement of Standard Tree Structure is that each node contain useful information. A node is defined to contain useful information if:

1.). It is at the lowest level (dimension 1) in the tree and contains one value.

2.) It is at the lowest level (dimension 1) in the tree and has at least one younger sibling that satisfies 1.)

3.) It is at level 2 or higher and one of its descendents ultimately leads to a node at the lowest level satisfying 1.)

4.) It is at level 2 or higher and has at least one younger sibling that satisfies 3. ). 


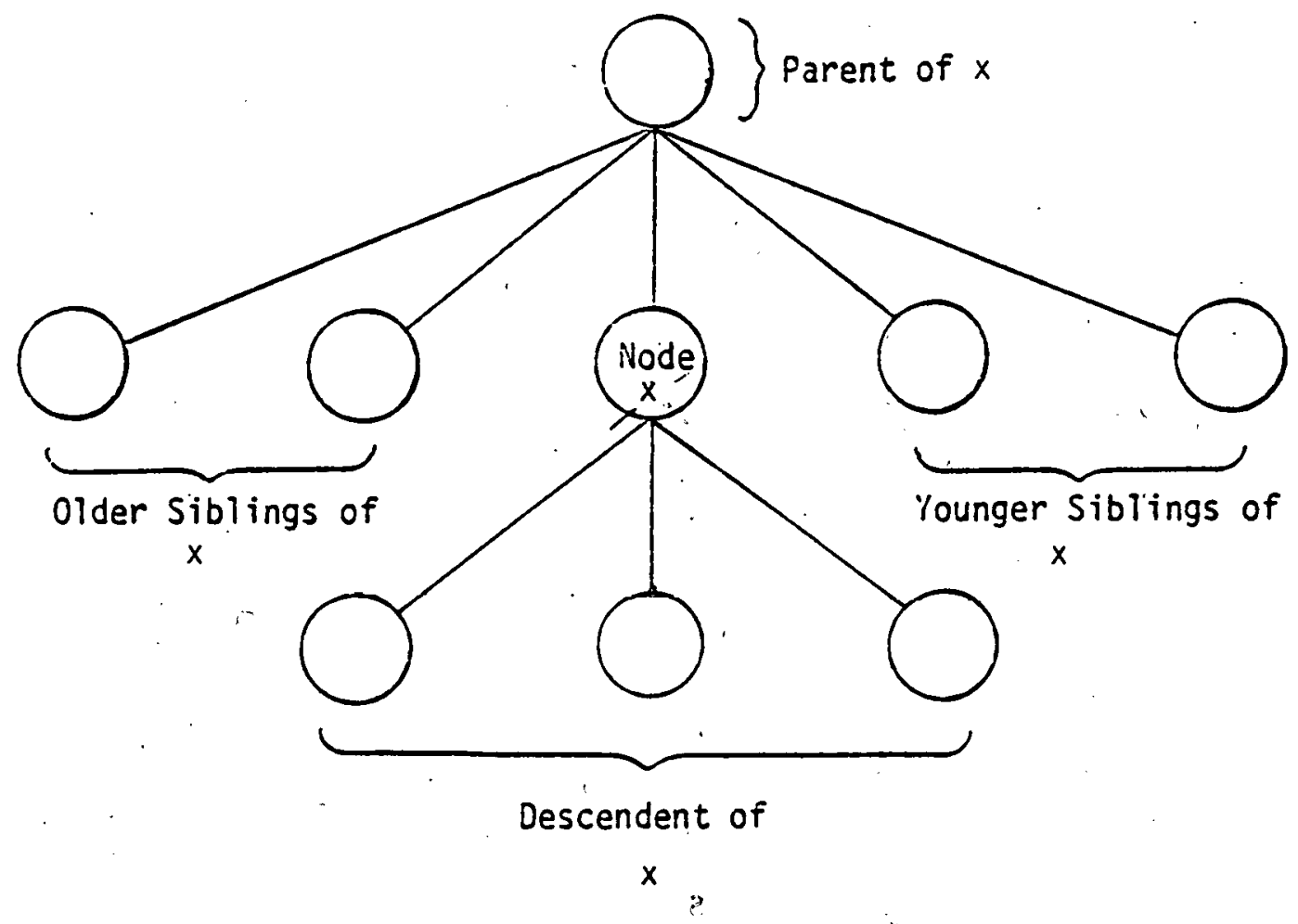

Figure III. B.4 HIERARCHICAL TREE TERMINOLOGY 
A node which has none of these properties represents no information and is called a null node. A null node can be defined recursively, starting at the first level. A node at the lowest level is a null node if it contains no values and has no younger siblings which contain values. A node at a higher level is a null node if it has no descendents which are not null nodes and has no younger siblings which are not null.

Figure III.B.5 illustrates the concept of null nodes. Nodes $M, N$, and $P$ are null because they reside on the lowest level, contain no values, and have no non-null younger siblings. Even though node $k$ contains no value, it has a non-null younger sibling $L$, and is thus not null. At the level above, node $J$ is null since it has no descendents and no younger siblings. Node $F$ is null, since its only descendents, $M$ and $N$ are both null, and node $I$ is likewise null.

The equivalent tree with the null nodes removed is shown in figure III. B.6 Note that even though node $K$ contains no values, it has a younger sibling, $L$, which is not null and is thus not null itself. In essence, it serves as a "place holder" for node $L$. Similarily, though nodes $C$ and $G$ have no descendents, they have non-null younger siblings and are thus not null themselves.

The other type of information which a node can contain is a name. Standard Tree Structure allows at most one name to be associated with any node. Any node which contains a name is not considered null. Thus, the only type of node which is forbidden by Standard Tree Structure is one that :

1. Contains no value (at the first level).

2. Has no non-null descendents.

3. Has no non-null younger siblings.

4. Contains no name. 


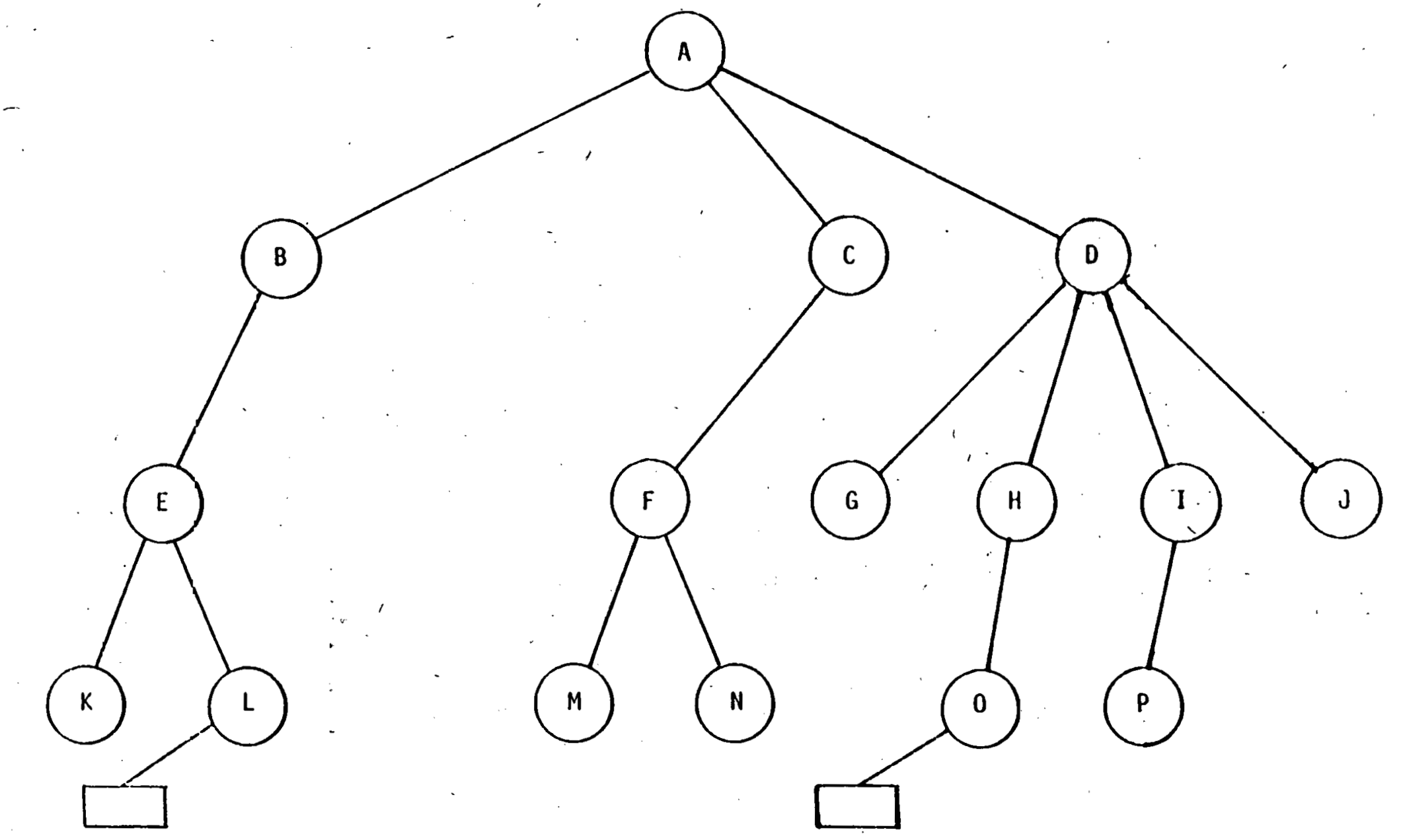

Figure IIJ.B.5: TREE HITH NULL NODES 


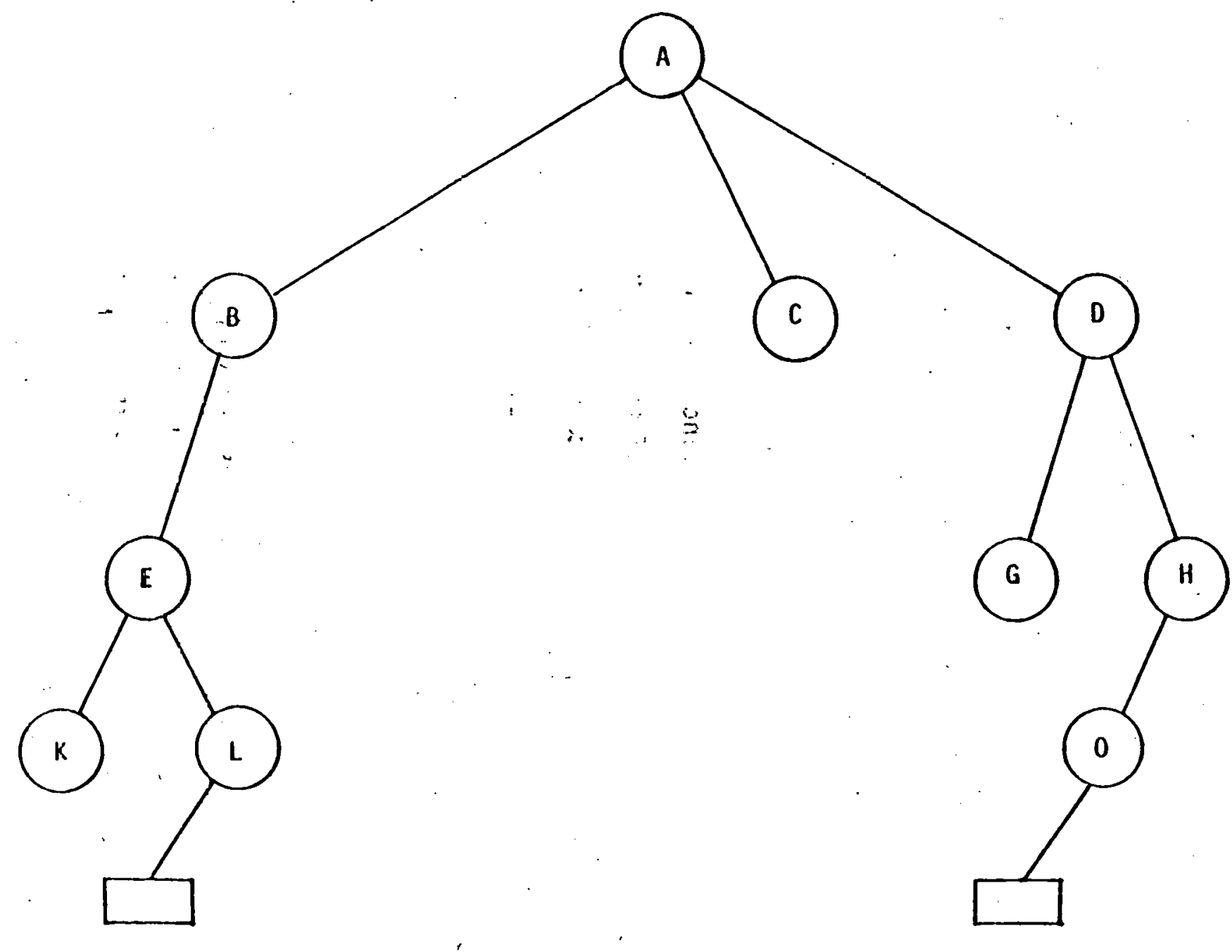

Figure III.B.G: SAME TREE IN STANDARD TREE STRUCTURE 
Any node that meets all four conditions is disallowed by Standard Tree Structure.

\section{Traversing the Tree}

Referring to the tree in Figure III. B. 3 we will now proceed to explain the algorithm by which we traverse the tree. In the data transfer system, the tree is actually traversed twice, both times according to the algorithm discussed below.

Recalling the discussion above where we characterized a node as occupying a unique position in the tree, we now wish to specify mathematically how we record that position. We will define the so-called IDV vector. as follows:

$$
\begin{aligned}
& \operatorname{IDV}\left(n_{\text {DIM }}\right)=\text { index of the descendent branch taken at the root of } \\
& \text { the tree (i.e. the index of the node visited at the } \\
& n_{\text {OIM }}{ }^{\text {th }} \text { level). According to our indexing convention } \\
& \operatorname{IOV}\left(n_{D I M}\right)=0 \text { if we have taken the "independent of } \\
& \text { dimension "OIM" branch. } \\
& \operatorname{IDV}\left(n_{\text {DIM }}-1\right)=i n d e x \text { of the descendent branch taken at the } n_{\text {DIM }} \\
& \text { dimension in the tree given IDV }\left(n_{D I M}\right) \\
& \operatorname{IDV}\left(n_{D I M}-2\right)=\text { index of the descendent branch taken at the } n_{D I M}{ }^{-1} \text {. } \\
& \begin{aligned}
\vdots & \text { dimension in the tree given } \operatorname{IDV}\left(n_{D I M}\right) \text { and } \operatorname{IDV}\left(n_{D I M}-1\right) \text {. } \\
\operatorname{IOV}(j)= & \text { index of the descendent branch taken at the } j+1 \text { st }
\end{aligned} \\
& \text { dimension in the tree given } \operatorname{IOV}\left(n_{\text {OIM }}\right), \ldots, \operatorname{IDV}(j+1) \text {. }
\end{aligned}
$$


Hence the position of the particular node at the $j^{\text {th }}$ dimension in the tree is uniquely specified by settings of the IOV vector for levels equal to and above $j$ :

$$
\begin{aligned}
& \operatorname{IDV}\left(n_{D I M}\right) \\
& \operatorname{IDV}\left(n_{D I M}-1\right) \\
& \vdots \\
& \operatorname{IDV}(j)
\end{aligned}
$$

The reader will note that settings of IDV at levels $j-1, \ldots, 2,1$ are unnecessary when we wish to point to a node at the $j^{\text {th }}$ level.

Given this position vector IDV, which allows us to uniquely specify any position in the tree, we now discuss the particular algorithm used to traverse the hierarchical tree. By traversing the tree, we mean visiting every node in the tree in a structured way.

The tree traversal algorithm used in the data transfer system to traverse the tree in FigureIII. B.I is illustrated in Figure III. B.7 The algorithm is "lexicographic" and is surmarized as follows:

1.) Visit the oldest descendent of the present node.

2.) If 1.) is impossible, visit the next younger sibling.

3.) If 1.) and 2.) are impossible, visit the parent node and try 2.).

4.) If 1.), 2.), and 3.) are impossible, visit the parent of the parent node and try 2.)

5.) If $1.1,2.), 3 . j$, and 4.) are impossible, vistt the parent of the parent of the parent node and try 2.)

This algorithm will visit every node in the tree and will in effect number the nodes successively beginning. with 1 as shown in the figure. 


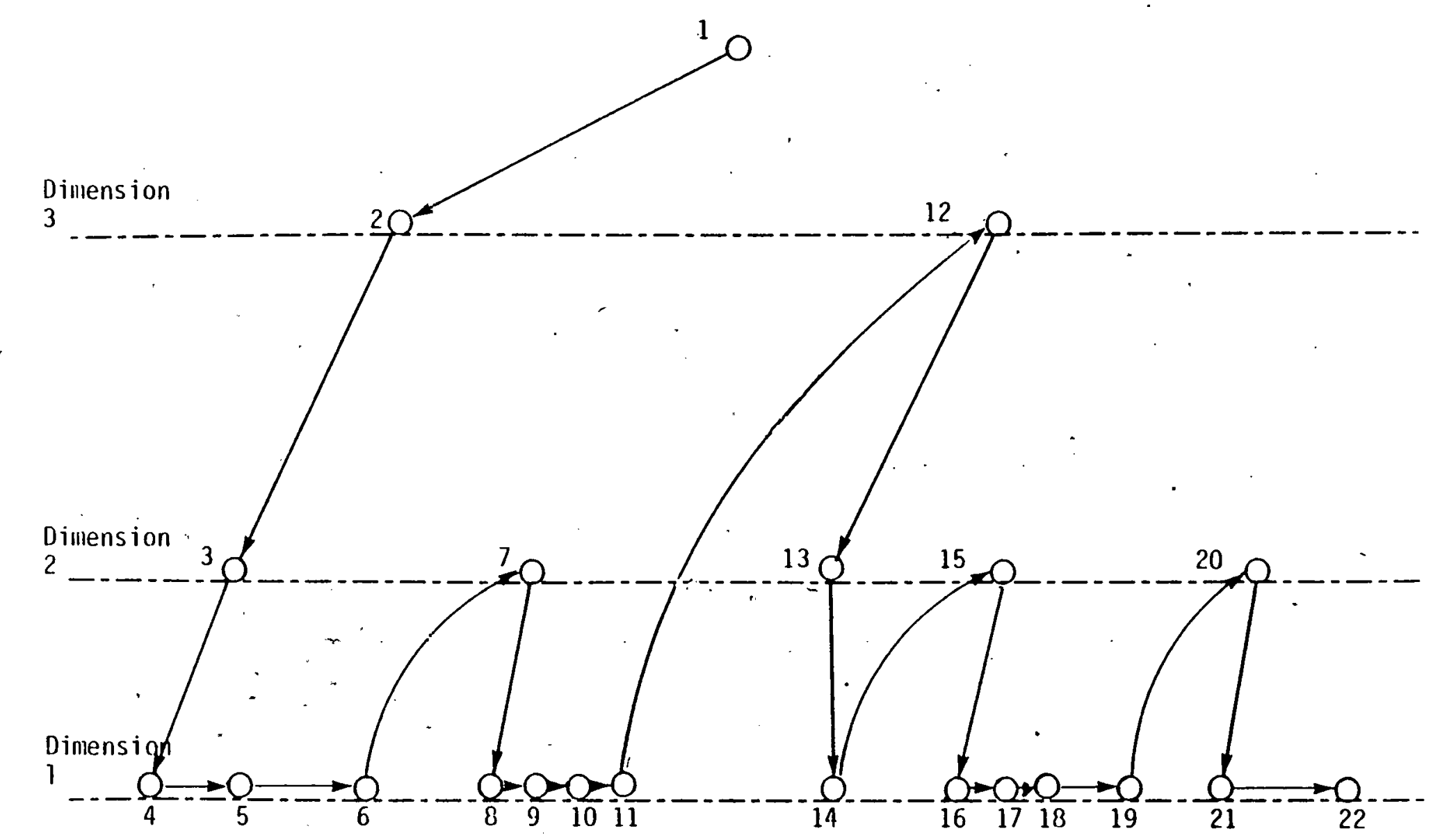

Figure III.B.7: TंRAVERSAL ALGORITHII FOR HIERARCHICAL TREE 


\section{Storage Versus Access Tradeoff}

For purposes of the present discussion, we will ignore all data stored at levels $2,3, \ldots, n_{\text {OIM }}$ and focus on the data stored at level 1 . The present version of the data transfer system makes this assumption -all numerical data is stored at the attribute level. We will begin this section by describing a highly storage efficient method of accessing the data on the terminal nodes and note its access inefficiency. We will then use this as a basis to motivate the design of the DFI data transfer system in which we have given up some storage efficiency in exchange for efficiency of data access.

A highly storage-efficient method of storing the data base would be to construct a vector $\underline{x}$ with a single position for every teminal node and to access the appropriate element in the vector $\underline{x}$ using the position vector IDV. We will briefly describe how this can be accomplished. We will discuss this in the context of Figures III.B.3 and III.B.7.

Suppose during our traversal of the tree according to the algorithm in Figure III.B.7, we had filled a vector $\underline{x}$ with the data values on the terminal branches in the order they are encountered. Upon completion of the tree traversal, the vector $\underline{x}$ would contain the following:

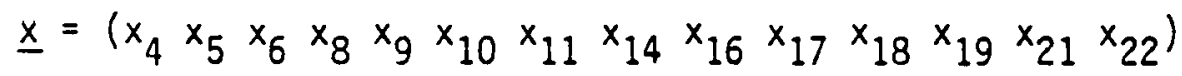

where $x_{i}$ is the value on the terminal node numbered $i$. Suppose further that the information in Table III.B.] had been stored during the traversal 
Table III.B.]

Node

Number

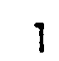

2

3

4

5

6

7

8

9

10

11.

12

13

14

15

16

17.

18

19

20

21

22

\section{1 dest \\ Descendent}

2

3

4

1

2

3

8

4

5

6

7

13

14

8

16

9

10

11

21

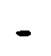

First Younger

Sibling

12

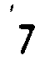

5

6
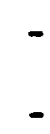

9

10

11

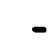

15

20

17

18

19

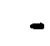

22 
as well. The table contains two lists. The first list specifies for each node in the tree the node number of the oldest descendent of that node. Because nodes at level 1 have no descendents, no entry will appear for level 1 nodes in this list. The second list specifies for each node in the tree the node number of the first younger sibling of that node. Nodes which are the youngest sibling will have no entry in this list. We will now show that the two lists have enough information to perform the following two functions:

1.) Given a position vector IDV that points to a particular terminal node, access the value attached to that node in the vector $\underline{x}$.

2.) Given an entry in the vector $x$, find the position vector IDV that points to that data value.

We will briefiy illustrate the necessary calculations because we wish to illustrate the number of calculations required for each of the two procedures.

First, suppose we are given the IDV vector

$$
\begin{aligned}
& \operatorname{IDV}(3)=1 \\
& \operatorname{IDV}(2)=1 \\
& \operatorname{IDV}(1)=3
\end{aligned}
$$

which, as we can see, points to terminal node 18. (This IDV vector is consistent with our node numbering conventions).

Beginning at leve] $3[\operatorname{IDV}(3)=1]$, we know that we must move over to the first "dependent on dimension 3 " branch at level 3. We can do this using the lists in Table III.B.I as follows. Beginning at the root 
(node 1) we move down to node 2 , which we know corresponds to $\operatorname{IDV}(3)=0$. This is the first branch at level 3. We know that we must move over 1 sibling so we look up

$$
\text { first younger sibling }(2)=12
$$

Hence node 12 lies along our path specified by IDV at level 3.

Having finished with level 3 , we then drop down to-level 2 , which is accomplished by finding the oldest descendent of node 12:

$$
\text { oldest descendent }(12)=13 \text {. }
$$

This corresponds to $\operatorname{IDV}(2)=0$. Since we want $\operatorname{IDV}(2)=1$, we mus.t move over 1 sibling at the 2 level as follows:

$$
\text { first younger sibling }(13)=15 \therefore \vdots \text { : }
$$

Hence node 15 lies along the path specificed by IDV at level 2.

Having finished with level 2, we then drop down to level 1 by finding the oldest descendent of node 15:

$$
\text { oldest descendent }(15)=16 \text {. }
$$

This corresponds to $\operatorname{IDV}(1)=1$. Remember by convention the numbering at level 1 starts at 1 . Since we want IOV $(1)=3$, we must move over 2 siblings as follows

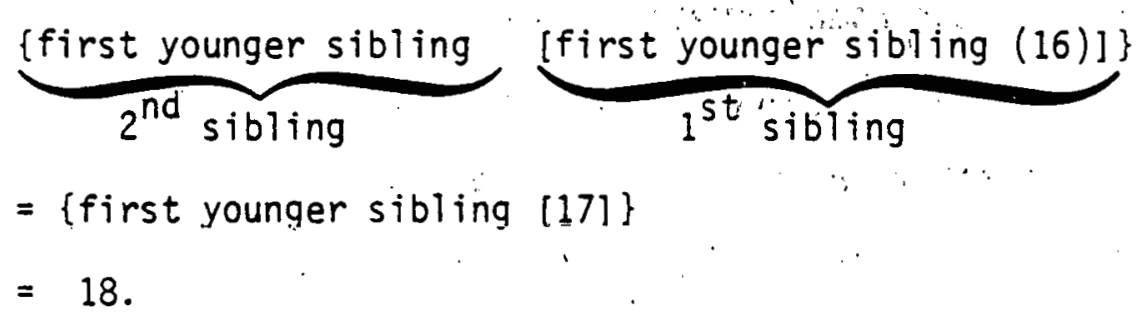


The value $x_{18}$ lies in the $11^{\text {th }}$ position in the $x$-vector, which can be found by counting down to the $11^{\text {th }}$ dash in the oldest descendent list. The reader will note that this simple calculation required

Four first younger sibling calculations

Two oldest descendent calculations.

The inverse calculation, that of calculating the path through the tree given the position in the vector $\underline{x}$, is of similar complexity. Suppose we have specified the $11^{\text {th }}$ position in the $\underline{x}$ vector. The first calculation we make is to step down the oldest descendent list until we get to the $11^{\text {th }}$ position, which occurs at node 18 . We wish to move first to the oldest sibling at our present level. Hence we ask "18 is the first younger sibling of what node?" That is,

$$
18 \text { = First younger sibling }(n) \text {. }
$$

The answer is $n=17$. We then ask a similar question of 17:

$$
17 \text { = First younger sibling }(n) \text {. }
$$

The answer is $n=16$. We then ask the same question of 16 :

$$
16 \text { = First younger sibling }(n) \text {. }
$$

Node 16 does not appear in the first younger sibling list, so we know that 16 is the oldest sibling at our present leve1. Furthermore 17 and 16 are older siblings of 18 and hence

$$
\operatorname{IDV}(\ell)=\left\{\begin{array}{l}
3 \text { if } \ell \text { turns out to be level } 1 \\
2 \text { if } \ell \text { turns out to be level } 2, \ldots
\end{array}\right.
$$


Knowing that 16 is the oldest sibling at our present level, we then ask "of what node -is 16 the oldest descendent?" The answer is found by locating the position of node 16 in the oldest descendent list:

$$
16=01 \text { dest descendent }(n) \text {. }
$$

The answer is $n=15$. We now ask whether node 15 has an older sibling:;

$$
15 \text { = First younger sibling }(n) \text {. }
$$

The answer is found to be $n=13$. We ask whether node 13 has an older sibling:

$$
13=\text { First younger sibling }(n)
$$

and find that node 13 does not appear in the first younger sibling table, implying that node 13 is an oldest sibling. Hence node 15 has one older sibling and we have

$$
\operatorname{IDV}(\ell+1)=1
$$

according to our branch numbering conventions.

Finally 13 must be the oldest descendent of some node $n$ :

$$
13=01 \text { dest descendent }(n) \text {. }
$$

The answer is $n=12$. Since

$$
12 \text { = First younger sibling }(n=2)
$$

and

$$
2=01 \text { dest descendent }(n=1) \text {, }
$$

we know that

$$
\operatorname{IDV}(\ell+2)=1 \text {. }
$$


Since we know we have hit the root, $\ell$ must equal 1 and

$$
\begin{aligned}
& \operatorname{IDV}(3)=1 \\
& \operatorname{IDV}(2)=1 \\
& \operatorname{IDV}(1)=3
\end{aligned}
$$

The reader will note that we required

Six first younger sibling calculations

Two oldest descendent calculations

to construct IDV.

The access method we have just discussed is highly general and capable of dealing with hierarchical trees with any branch structure. The computational efficiency of the method depends on the number of siblings at the various levels -- extremely "bushy" trees being less efficient.

This method will be quite powerful in the event we have a high degree of conditionality in the hierarchical tree, that is when the number of descendent branches at each level is strongly dependent on all or most of the higher level branches we have come down. A good deal of simplification is possible, however, when the number of. descendent branches at each level is strongly dependent on only a few. of the higher level branches and weakly dependent on the res.t. . In most practical applications, model data bases tend to display the latter characteristic -- the number of nodes at a given level depends strongly on just a few higher level dimensions and weakly or not at all on most of the higher level dimensions. In this case, we should be 
able to give up a small amount of storage efficiency in return for a large amount of computational efficiency. The DFI data transfer system is based upon this design criterion.

\section{Sets}

The first simplification that can be made in a general hierarchical tree is to exploit the concept of sets defined above: 1 A set can be viewed as a sub-tree of the total hierarchical tree that contains nothing but "dependent on dimension $j$ " branches. Because these set sub-trees by definition contain fewer than $n_{D I M}$ dimensions, the number of calculations in any tree traversal scheme can be reduced by simply knowing which set (which sub-tree) is to be traversed and traversing only that sub-tree.

The tree traversal algorithm used in the DFI data transfer system decomposes the general hierarchical tree into sub-trees corresponding to the sets. By so doing, the data is rearranged so that it can easily be accessed by set, thus eliminating the necessity to consider most of the data base when we are attempting to access a particular data value. The method by which this can be accomplished is simple. Given the position vector IDV, we can determine in which set the path described by IDV lies -- the non-zero dimensions of IDV uniquely specify that set. Hence we can immediately suppress all zero elements of IDV by simply knowing in which set the path specified by IDV lies. Although the computational saving of using sets as our fundamental data structure is not large, the conceptual advantages are significant as we shall see shortly.

1. In Chapter III, the concept of a data set was introduced. We have shortened the term to set for use in this section. 


\section{Square Trees}

The second simplification that can be made in a general hierarchical tree occurs when the tree 'is square -- if the number of descendents at each level is unconditional on all higher dimensions and hence there are an equal number of descendents from all nodes at the same level. Figure III.B.8 illustrates a three-level square tree. The nodes have been numbered as if they had been visited by the tree traversal algorithm illustrated in Figure III.B.7 Note that there are
4 descendents from the root
2 descendents from each node at level 3
3 descendents from each node at level 2 .

In this square tree, we can quickly point to any data element at a terminal branch given the position vector IDV. For example, given

$$
\begin{aligned}
& \operatorname{IDV}(3)=3 \\
& \operatorname{IOV}(2)=1 \\
& \operatorname{IDV}(1)=2,
\end{aligned}
$$

the index of the corresponding terminal node is!

$$
\begin{array}{ll} 
& 3 \times[2 \times 3] \\
+ & 1 \times[3] \\
+ & 2 \\
=\quad & \operatorname{IDV}(3) \times[2 \times 3] \\
+ & \operatorname{IDV}(2) \times[3] \\
+ & \operatorname{IDV}(1) \\
= & 23
\end{array}
$$

$T$ In the remainder of section $B$, we will confine our discussions to set sub-trees. All branches in set sub-trees are indexed $1,2, \ldots, n$, rather than $0,1,2, \ldots, n$ (for dimension 3 and higher) as in the complete hierarchical tree. This is consistent with the coding of the TRANSFER SYSTEM, which uses the set-decomposed tree. 


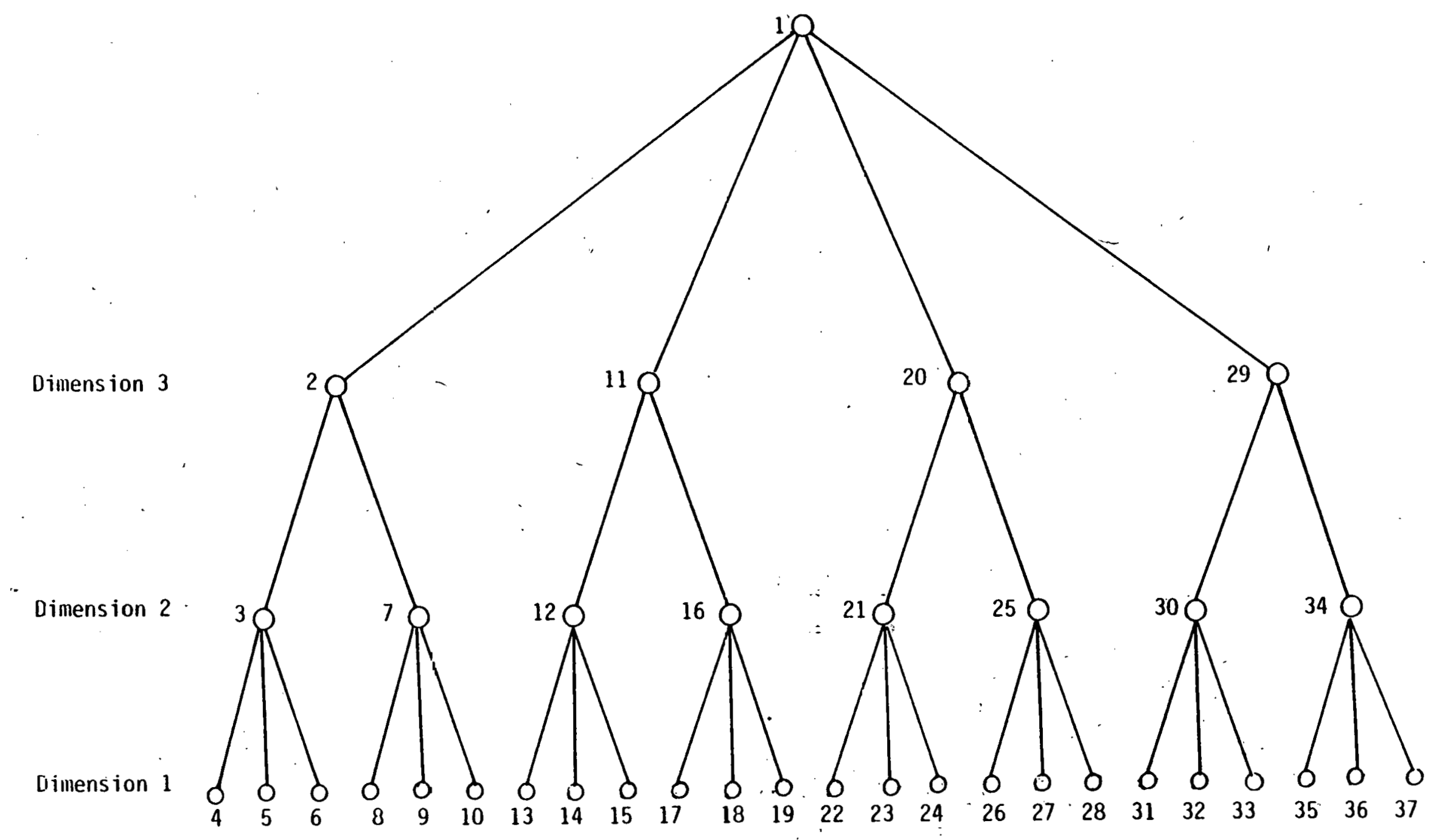

Figure III.B.8: SQUARE TREE 
The fact that indexing the terminal branches in a square tree is highly efficient is not surprising when we note that a square tree corresponds to a cube of dimensionality equal to the number of levels of the tree. The square tree in Figure III.B.8 corresponds to a $3 \times 2 \times 4$ cube as shown in Figure III.B.9. Hence, by decomposing the general hierarchical tree into square sub-trees, we can very quickly access any terminal value by computing its square set tree index as illustrated above.

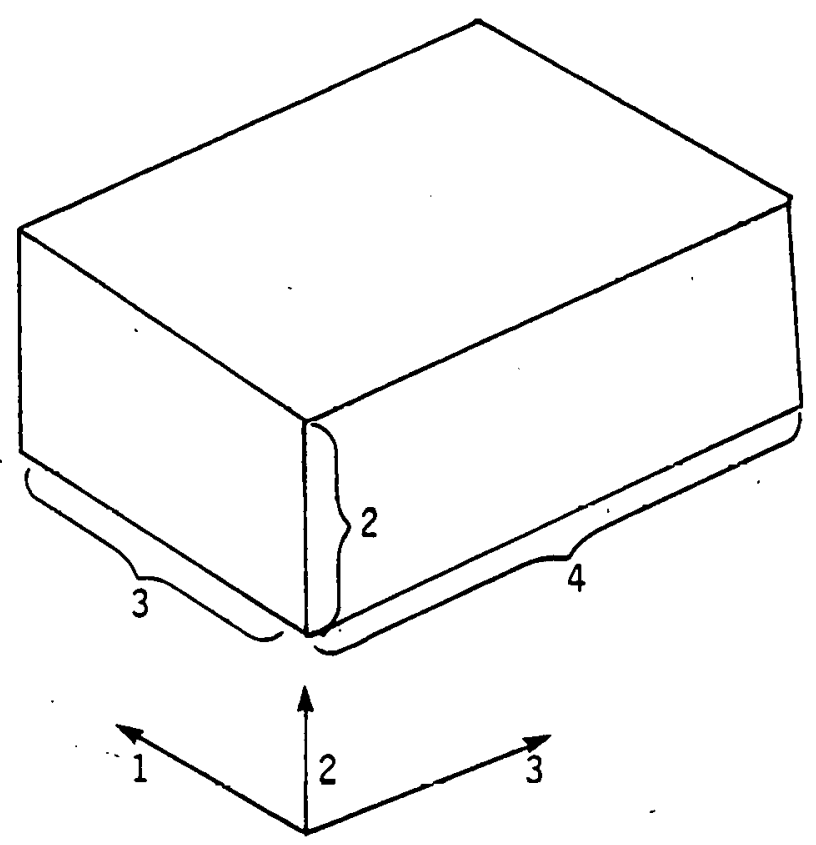

Figure III.B.9: CUBE REPRESENTATION OF SQUARE TREE 


\section{Single-Level Conditionality}

Summarizing our earlier discussions, the general node access system developed in conjunction with Table III.B.I represents one extreme -- the extreme of high storage efficiency at the expense of access efficiency. The square tree represents the other extreme -that of high data access efficiency. The former extreme is justified when the data base contains a high degree of conditionality, but the latter is justified when the data base has the minimum conditionality -the number of successor branches at a given level depends only on the current level.

Suppose now we "split the difference" between the two extremes by assuming that the number of successor branches from the $n$-th node at the $j$-th level is conditional on the setting of one branch at the $j$-th or higher level. That is, suppose the number of successor branches from the $n$-th node at the $j$-th level is conditional on which node we have visited at the $k_{. j}$-th level $\left(k_{j} \geq j\right)$. Using this assumption, we can still preserve some conditionality. in the tree but can eliminate a good deal of the conditionality, saving on access time. We will discuss this concept of single-level conditionality in some detail as it is central to the DFI data transfer system.

The discussion of single-level conditioning, which we refer to as storage conditionality, will begin using the example in Figure III.B.10. Suppose we simply "square-off" the tree in the figure by assuming that the number of successor branches from each node at level 3 is equal and is large enough to accommodate any of the fans at level 3. In order to accomplish this, we would have to add two successor branches to node $h$ 


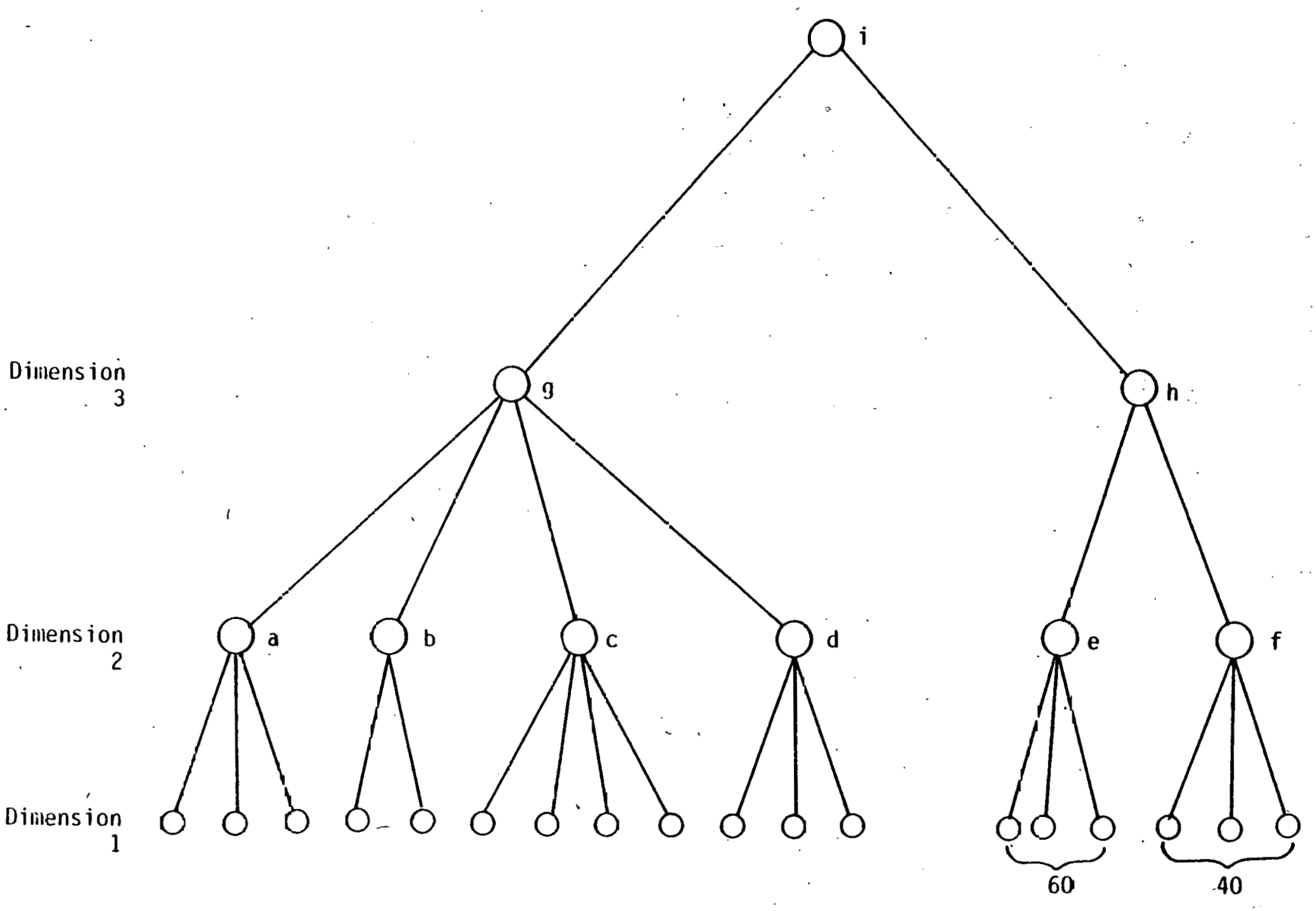

Figure [II. B.]0: EXAMPLE TREE -- STORAGE CONDITIONALITY 
so that it would have four successors like node $g$. Similarly for nodes at level 2, we would have to increase the size of all fans to 60 . The result would be the square tree shown in Figure III.B. 11 which is a $2 \times 4 \times 60$ tree.

The square tree in Figure III.B..11 is very efficient in terms of access time. For example, if we wish to access the data lying at the terminal branch

$$
\begin{aligned}
& \operatorname{IDV}(3)=1 \\
& \operatorname{IDV}(2)=3 \\
& \operatorname{IDV}(1)=3
\end{aligned}
$$

we would use the simple square tree multiplication algorithm discussed above. However, the square tree is not storage efficient. It requires $2 \times 4 \times 60=480$ storage locations to store the 112 terminal values at the branch tips. in Figure IV.A.13. Because it will become important later, we will define

$$
\begin{aligned}
& n_{I}(j)=\text { number of immediate successor branches from nodes at } \\
& \text { level } j, j=2,3, \ldots, n_{D_{I M}}+1 \\
& n_{U}(j)=\text { number of ultimate successor branches from nodes at } \\
& \text { level } j, j=2,3, \ldots, n_{\mathrm{OIM}^{+}}+1 \text {. }
\end{aligned}
$$

In the example in Figure III.B. 11

$$
\begin{array}{ll}
n_{I}(2)=60 & n_{U}(2)=60 \\
n_{I}(3)=4 & n_{U}(3)=240 \\
n_{I}(4)=2 & n_{U}(4)=480
\end{array}
$$




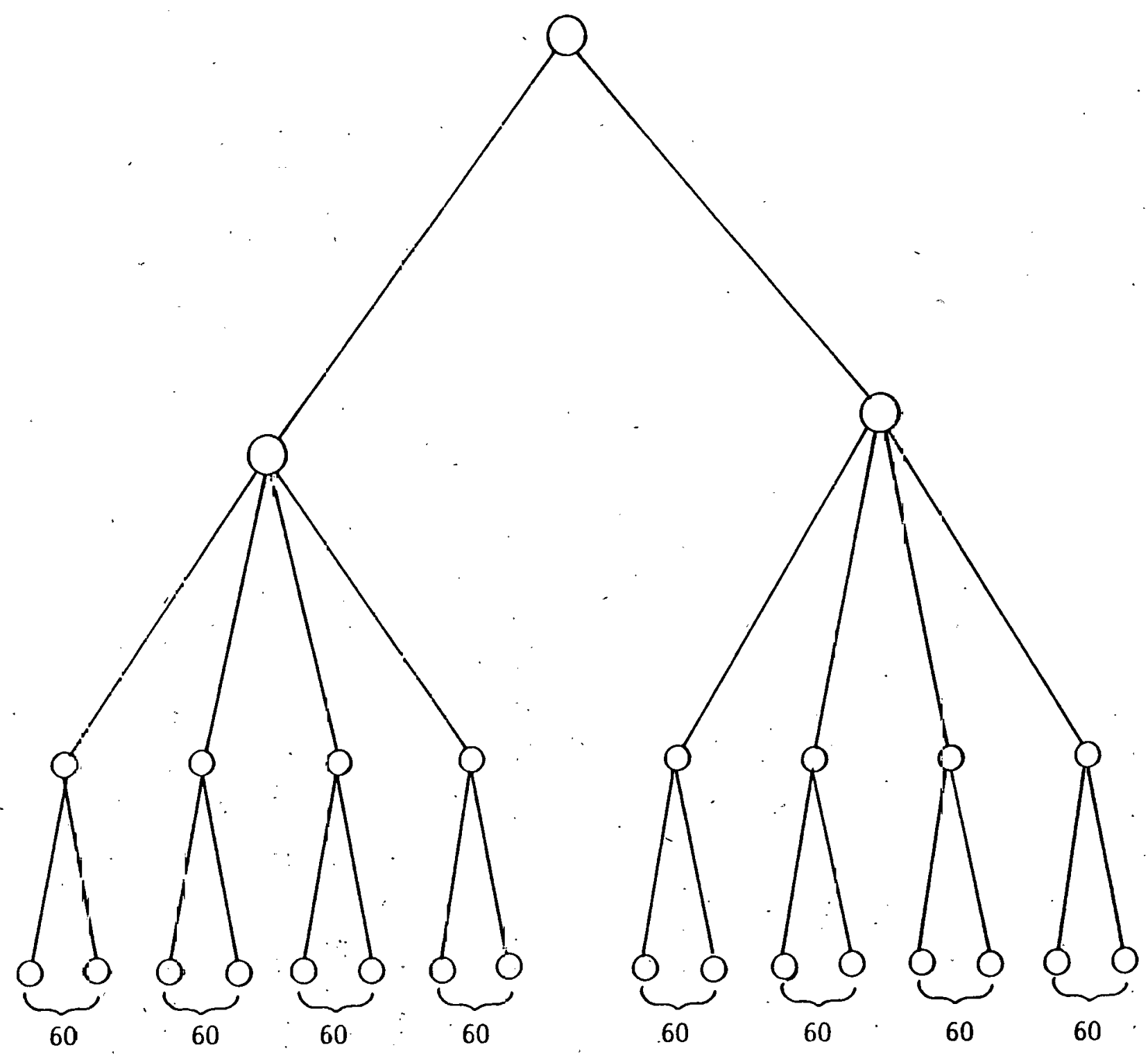

Figure III.B.11: EXAYPLE TREE AFTER SQUARING OFF AT ALL LEVELS 
Hence if we define a vector $\underline{d}$ of length 480 in which we place the terminal values in $2 \times 4=8$ groups of 60 , we can develop a simple method for indexing the terminal values in this $\underline{d}$ vector.

If we wish to access in the d-vector the number corresponding to

$$
\begin{aligned}
& \operatorname{IDV}(3)=1 \\
& \operatorname{IDV}(2)=3 \\
& \operatorname{IDV}(1)=3
\end{aligned}
$$

we know we must count over

3 positions for $\operatorname{IDV}(1)$

$(3-1) \times 60=(3-1) \times n_{U}(2)$ positions for IDV(2)

$(1-1) \times 240=(1-1) \times n_{U}(3)$ positions for $\operatorname{IOV}(3)$

$=123$ positions

We will extend this reasoning below to the case where we allow one level (rather than no levels) of conditionality on higher level dimensions.

Rather than the naive approach of "squaring off" the tree by adding the same number of descendent branches at each level unconditional on higher level values, suppose we instead let the number of descendent branches at the $j$-th level be dependent on the branch we came down at the $k_{j}$-th level $\left(k_{j} \geq j\right)$. Let $k_{j}^{*}$ denote the value (i.e. the branch setting) at the $k_{j}$-th level in the tree. In order to incorporate this additional generality, we will let: 


$$
\begin{aligned}
n_{I}\left(j, k_{j}^{*}\right)= & \text { number of immediate successor branches from nodes } \\
& \text { at the } j^{\text {th }} \text { level given that we came down the } k_{j}^{*} \text { th } \\
& \text { branch at the } k_{j}^{\text {th }} \text { level. } \\
n_{U}\left(j, k_{j}^{*}\right)= & \text { number of ultimate successor branches from nodes at } \\
& \text { the } j^{\text {th }} \text { level given that we came down the } k_{j}^{*} \text { th branch } \\
& \text { at the } k_{j}^{\text {th }} \text { level. }
\end{aligned}
$$

It should be emphasized that in order to use storage conditionality, the user must specify for each dimension $j$ the dimension $k_{j}$ upon whose value the number of successor branches at level $j$ will be assumed to be dependent.

We will illustrate the concept of storage conditionality using the sample tree in Figure III.B.10. We will make two different storage conditionality assumptions and note their implications for storage. To begin our discussion, we will assume that the number of successor branches:

at level 2 is conditional on the node visited at level 2 (i.e. $k_{2}=2$ )

at level 3 is conditional on the node visited at level 3 (i.e. $k_{3}=3$ ).

We begin our discussion by noting that there are two nodes at level 2 corresponding to the first successor branch from level 3 . They are a and $e$. In order to reserve enough space to store the larger of the two fans at a or $\dot{e}$, we must reserve

$$
\operatorname{MAX}\{3,60\}=60 \text { positions }
$$

Hence $\quad n_{I}\left(j=2, k_{2}^{\star}=1\right)=60$. 
Applying similar logic to nodes $b$ and $f$, which correspond to the second conditional branch at node 2 , we must reserve

$$
\operatorname{Max}\{2,40\}=40=n_{I}\left(j=2, k \frac{k}{2} 2\right)
$$

storage locations. For conditional nodes $c$ and $d$, we must reserve

$$
\text { and } \begin{aligned}
\operatorname{Max}\{4,0\} & =n_{I}\left(j=2, k_{2}^{*}=3\right)=4 \\
\text { Max }\{3,0\} & =n_{I}\left(j=2, k_{2}^{\star}=4\right)=3
\end{aligned}
$$

locations respectively. After this conditional maximization, we will have the tree shown in Figure III.B.12.

Moving up to level 3, suppose the number of immediate successor branches at level 3 is conditional on which node at level 3 we have visited. We then know that

$$
\begin{aligned}
& n_{I}\left(j=3, k_{3}^{*}=1\right)=4 \text { branches } \\
& n_{I}\left(j=3, k_{3}^{\star}=2\right)=2 \text { branches }
\end{aligned}
$$

Thus for purposes of storage, the expanded tree appears as in Figure III.B.12.1

Given the values of $n_{I}(\cdot, \cdot)$, we can calculate the ultimate number of successor branches $n_{U}\left(j, k_{j}^{*}\right)$ for each node. They are

$$
\begin{aligned}
& n_{U}\left(j=2, k_{2}^{+}=1\right)=60 \\
& n_{U}\left(j=2, k_{2}^{*}=2\right)=40 \\
& n_{U}\left(j=2, k_{2}^{*}=3\right)=4 \\
& n_{U}\left(j=2, k_{2}^{*}=4\right)=3 \\
& n_{U}\left(j=3, k_{3}^{*}=1\right)=107 \\
& n_{U}\left(j=3, k_{3}^{*}=2\right)=100
\end{aligned}
$$

and hence the total number of successor branches from the root is

$$
n_{U}\left(j=4, k_{4}^{*}=1\right)=207 \text {. }
$$

1: The dotted branches are not included. 


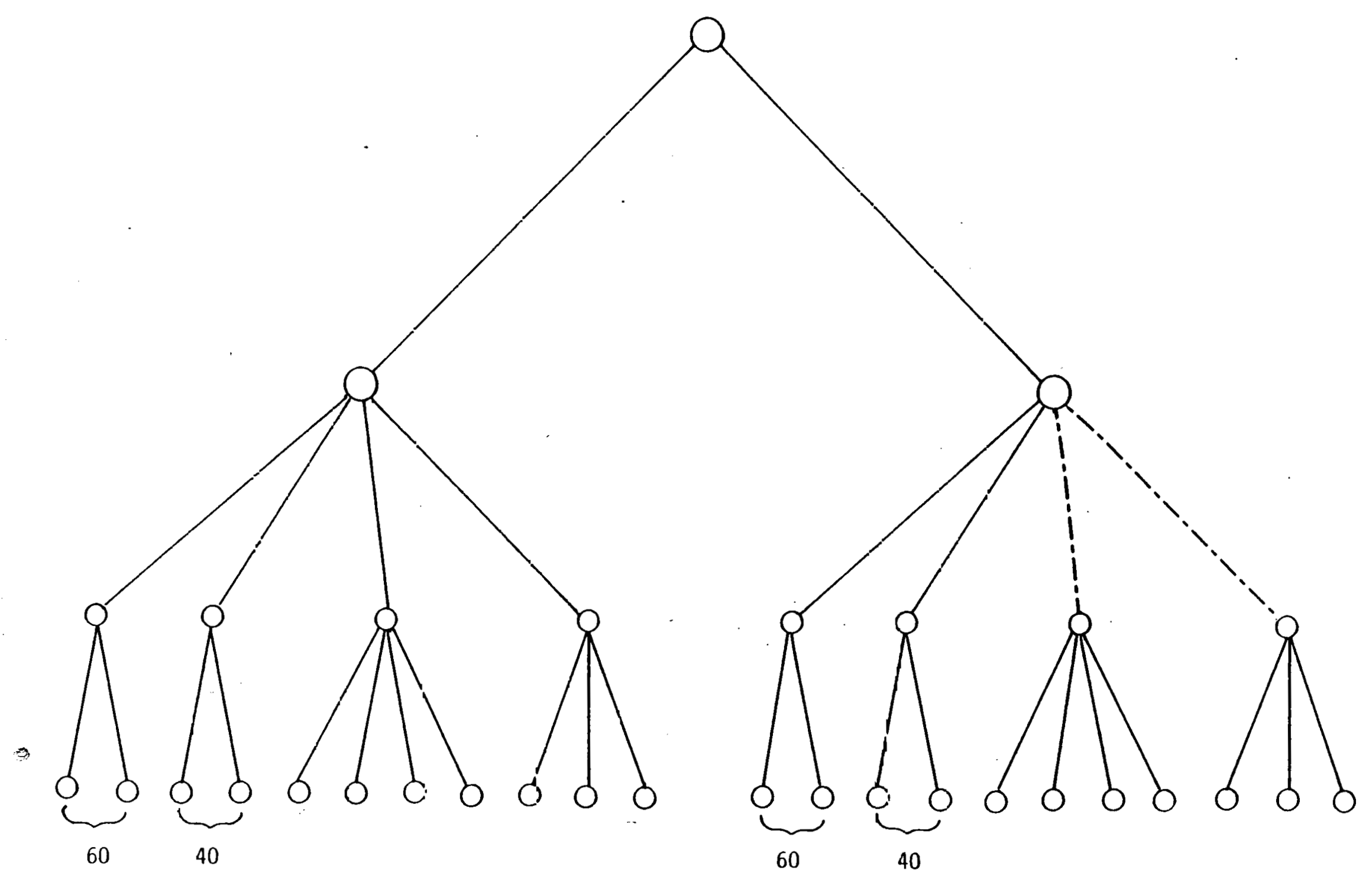

Figure III.B.12: EXAMPLE TREE -- STORAGE CONDITIONAL ON SAME LEVEL 
Using the tree in Figure III.B.12 we would require 207 storage positions. to access the 112 terminal values.

A natural way to allocate the necessary storage space is to define a vector of length 207 . We then partition the vector first into partitions of length 107 and 100 . We then partition the sub-vector of length 107 into sub-vectors of length $60,40,4$, and 3 and partition the subvector of iength 100 into sub-vectors of length 60 and 40 . This partitioning is shown in Figure III.B.13. If we store the values $n_{U}\left(j, k_{j}^{*}\right)$ we can easily locate the position in the vector $\underline{d}$ where a particular terminal value lies given the IDV vector that points to it. For example, let

$$
\begin{aligned}
& \operatorname{IDV}(3)=1 \\
& \operatorname{IDV}(2)=3 \\
& \operatorname{IDV}(1)=3
\end{aligned}
$$

We know that we must move over,

\section{0}

positions because we are accessing the first value IDV(1)! Moving down to level 2, we must move over another

$$
\begin{aligned}
n_{U}\left(j=2, k_{2}^{\star}=1\right) & =60 . \\
+ & n_{u}\left(j=2, k_{2}^{\star}=2\right)=40
\end{aligned}
$$

positions because we skipped $\operatorname{IDV}(2)=1$ and $\operatorname{IDV}(2)=2$. Moving down to level 1 , we must move over another

$$
\operatorname{IDV}(1)=3
$$

1. This example assumes the tree in Figure III.B.10 is a set tree whose branches are indexed $1, \ldots n$. The reasoning applies equally well, however, to multi-set hierarchical trees, i.e., torees where the branches are indexed $0,1, \ldots, n$. 


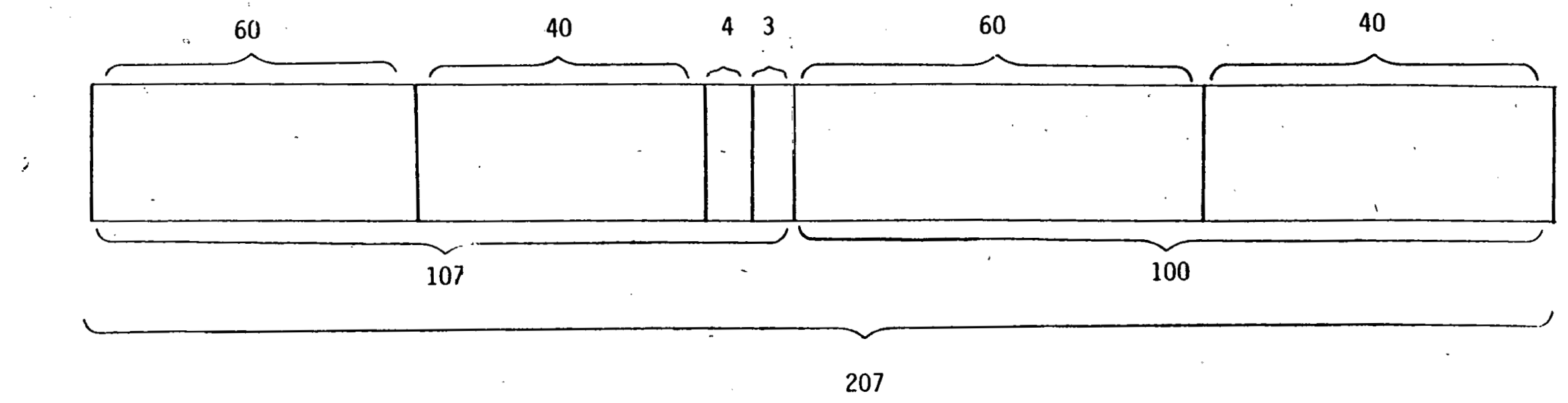

Figure III.B.13: Example d-vector -- Storage Conditional on Same Level 
positions because we skipped over positions 1 and 2 . Hence we know the corresponding value will be found in position $0+60+40+3=103$ in the vector $\underline{\mathrm{d}}$.

Returning to the tree in Figure III.B.10 suppose we now assume that the number of immediate successor branches at level. 2 is conditional not on our location at level 2 but instead our location at level 3 . Under this assumption, we know that we would require

$$
\operatorname{MAX}\{3,2,4,3\}=4=n_{I}\left(j=2, k_{2}^{*}=1\right)=n_{U}\left(j=2, k_{2}^{*}=1\right)
$$

storage positions under each node at level 2 under the first branch at level 3. Using similar logic for each node at level 2 under the second branch at level 3, we would requir

$$
\operatorname{MAX}\{60,40\}=60=n_{I}\left(j=2, k_{2}^{*}=2\right)=n_{U} \cdot\left(j=2, k_{2}^{*}=2\right)
$$

storage positions. Hence the conditional tree for this setting of storage conditionality appears as in Figure III.B.14. Assuming the number of ultimate successor branches for nodes at level 3 is conditional on the branch we have taken at the root, we know that

$$
\begin{aligned}
& n_{U}\left(j=3, k_{3}^{*}=1\right)=4 \times 4=16 \\
& n_{U}\left(j=3, k_{3}^{\star}=2\right)=2 \times 60=120
\end{aligned}
$$

and hence the total number of branches indicated at the root is

$$
n \cdot\left(j=4, k_{4}^{\star}=1\right)=136
$$

to store the 112 terminal values. The partitioning of the d-vector needed to store this conditional tree is shown in Figure III.B.15. 


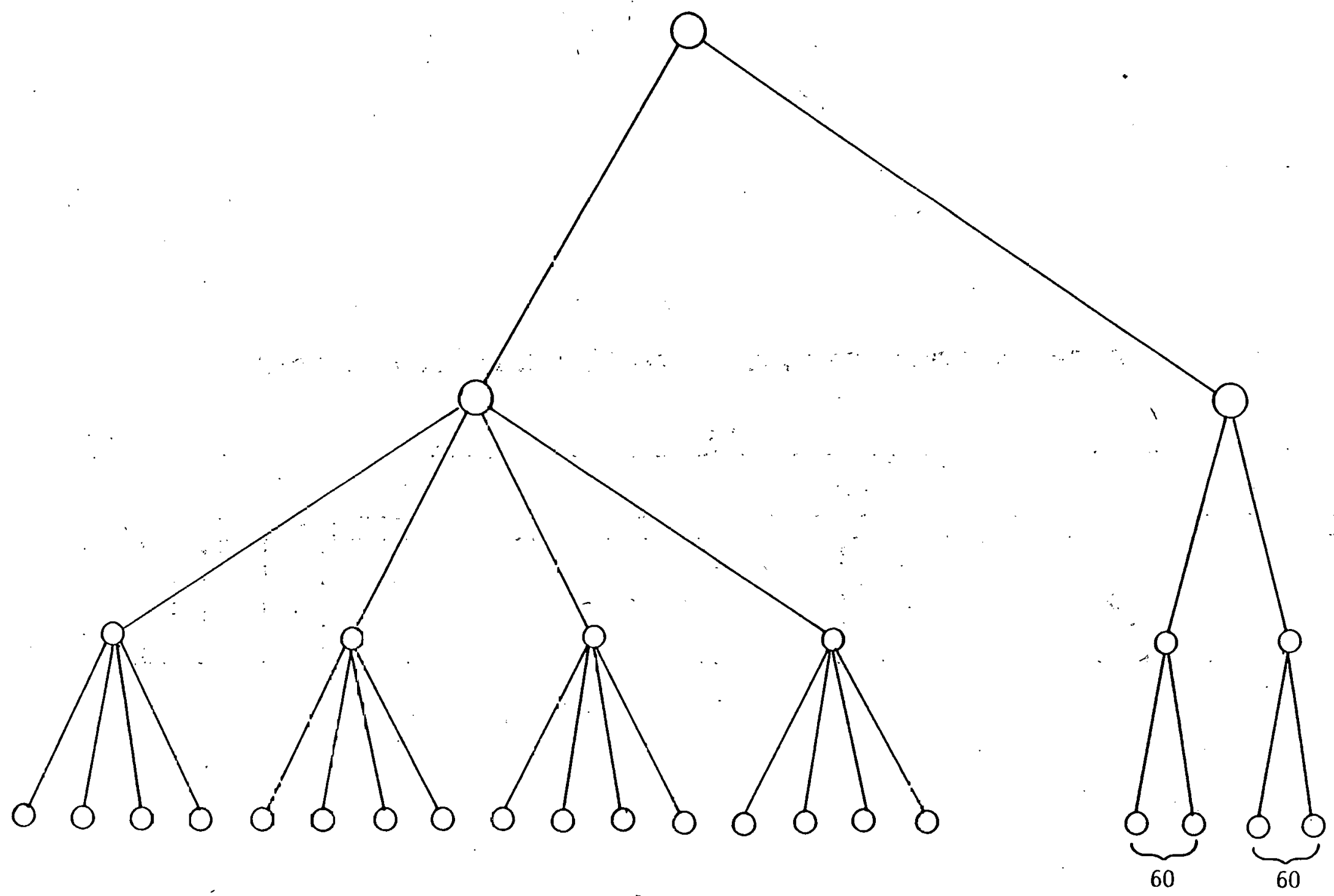

Figure III.B. 14: EXAMPLE TREE -- STORAGE CONDITIONAL ON HIGHER LEVEL 


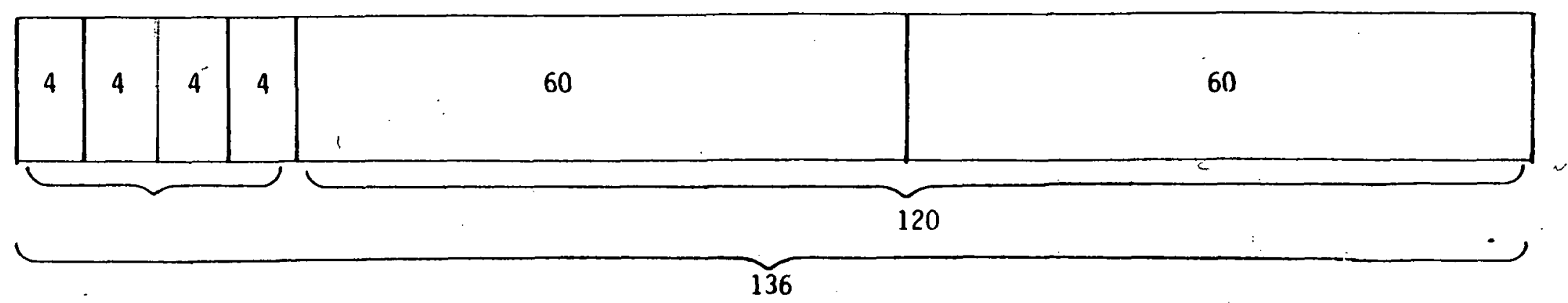

FIGURE III.B. 15: EXAMPLE d-vector -- STORAGE CONDITIONAL ON HIGHER LEVEL 
In this example, we can look up the value corresponding to

$$
\begin{aligned}
& \operatorname{IDV}(3)=1 \\
& \operatorname{IDV}(2)=3 \\
& \operatorname{IDV}(1)=3
\end{aligned}
$$

as follows. We move over

$$
0
$$

positions at level 3 because we are accessing the first value IDV(1). Moving down to level 2, we must move over

$$
2 \times n_{U}\left(j=2, k_{2}^{*}=1\right)=2 \times 4=8
$$

positions because we came down branch $1=k_{2}^{*}$ at level 3 and because we skipped over positions 1 and 2 at level 2. Finally we must move over another $\operatorname{IDV}(1)=3$ positions at level 1 , for a total of

$$
0+8+3=11
$$

positions to access the desired value.

Note that if we have saved the values of $n_{U}(\cdot, \cdot)$, we can save the potentially lengthy series of table lookups described in Section III.B.3 and can thus increase the computational efficiency of accessing the data. 
7. Algorithm for Retrieving/Storing Values

In this section, we will develop a method for traversing a tree whose storage is described by "offsets" (i.e. single level conditionality) and storing the values at $i$ ts terminal branch nodes in a single vector. The discussion will be conducted in the context of the tree in Figure III.B.10. Recalling the development leading to Figure III.B.13 in which we assumed "self-conditionality", the following conditional offsets were generated

$$
\begin{aligned}
& n_{U}\left(j=2, k_{\frac{\pi}{2}=1}^{*}=60\right. \\
& n_{U}\left(j=2, k_{2}^{\star}=2\right)=40 \\
& n_{U}\left(j=2, k_{2}^{\star}=3\right)=4 \\
& n_{U}\left(j=2, k_{2}^{\star}=4\right)=3 \\
& n_{U}\left(j=3, k_{3}^{\star}=1\right)=107 \\
& n_{U}\left(j=3, k_{\frac{\pi}{3}}^{*}=2\right)=100 \\
& n_{U}\left(j=4, k_{\frac{\pi}{4}}^{\star}=1\right)=207
\end{aligned}
$$

Assuming we wish to access the value corresponding to the path

$$
\begin{aligned}
& \operatorname{IDV}(3)=l_{3} \\
& \operatorname{IDV}(2)=l_{2} \\
& \operatorname{IDV}(1)=l_{1},
\end{aligned}
$$

we can develop the following algorithm. Beginning with level 3 in the tree, if $\ell_{3}=1$, we know we are in the first of the $3^{\text {rd }}$ level offsets and if $\ell_{3}=2$ we are in the second. Hence we must move over to the right the distance, 


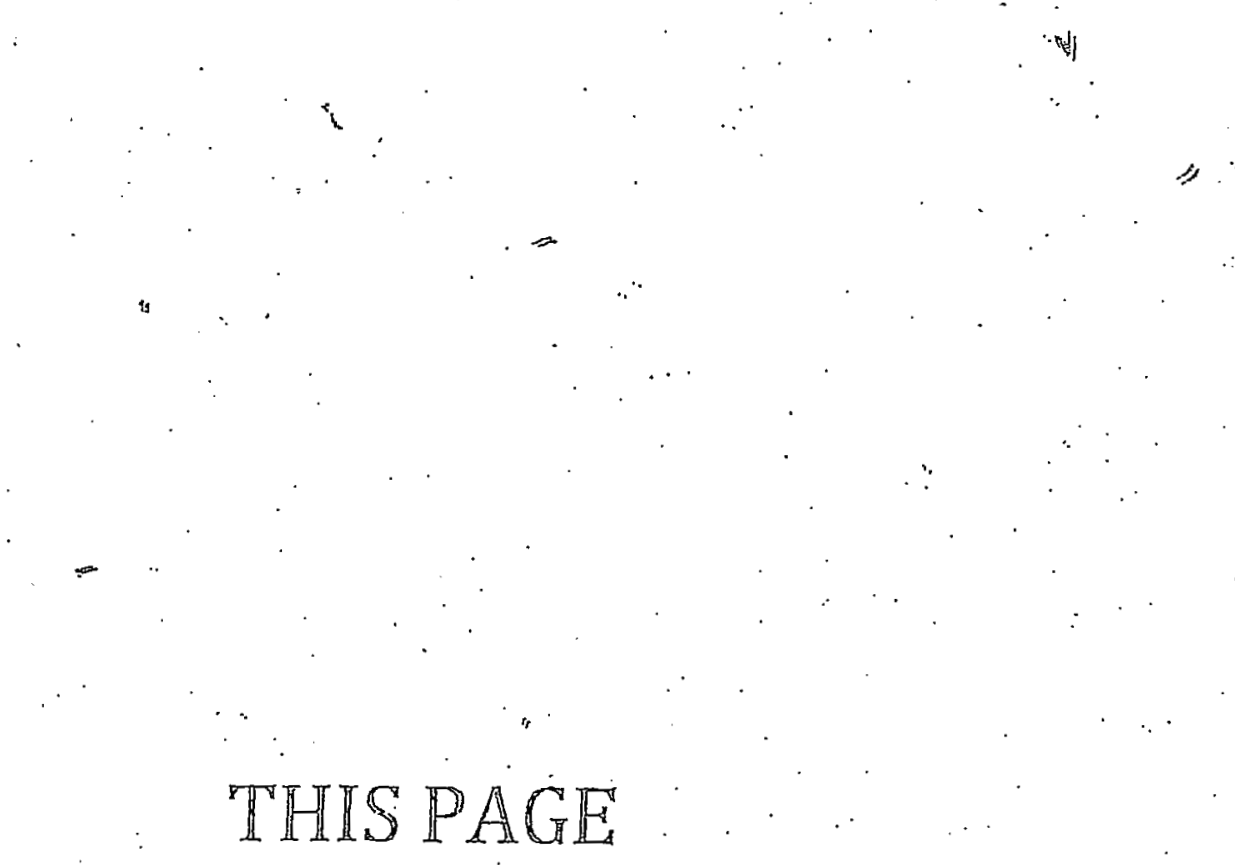

WAS INTENTIONALLY

LEFT BLANK 


$$
\text { .. } n_{3}=n-n_{U}\left(j=3, k_{3}^{\star}=1\right)-n_{U}\left(j=3, k_{3}^{\star}=2\right)
$$

and test whether the result is strictly positive. If so, we know that $\operatorname{IDV}(3) \geq 3$. If not, $\operatorname{IDV}(3)=2$. We continue subtracting successive values of $n_{U}($.$) from n$ until we get a non-positive number. This determines $\operatorname{IDV}(3)$. We then add the last value $n_{U}\left(j=3, k_{3}^{*}=k\right)$ back into the difference to give a positive number $n_{2}$.

We then begin at level 2 by computing

$$
n_{2}-n_{U}\left(j=2, k_{2}^{\star}=1\right)
$$

and checking whether it is strictly positive. If so, we know that $\operatorname{IDV}(2) \geq 2$. If not, $\operatorname{IDV}(2)=1$. If positive, we subtract $n_{U}\left(j=2, k_{2}^{*}=2\right)$ to obtain

$$
n_{2}-n_{U}\left(j=2, k_{2}^{\star}=1\right)-n_{U}\left(j=2, k_{2}^{*}=2\right)
$$

and check whether it is strictly positive. If so, we know that $\operatorname{IDV}(2) \geq 3$, if not $\operatorname{IDV}(2)=2$. We continue until we have determined $\operatorname{IDV}(2)$. We then add back in the final offset $n_{U}\left(j=2, k_{2}^{*}=k\right)$ and compute $n_{1}$.

When we know that we have reached level 1 , we know that $\operatorname{IDV}(1)=n_{1}$. In the case where we assume that the offsets at level 2 are conditional on level 3 and the offsets at level 3 are self-conditional, the same algorithm applies. However the term $s_{2}$ becomes

$$
s_{2}=\left\{\begin{array}{l}
0 \quad \ell_{2}=1 \\
\left(l_{2}-1\right) n_{U}\left(l=2, k_{2}^{\star}=1\right) \quad l_{2} \geq 2
\end{array}\right.
$$

because the offsets at level 2 are conditional on the value at level 3 . 


\section{DATA TRANSFER: FILLING A LABELLED COMMON}

The purpose of this section is to build on the previous sections to develop a method for moving data from the data base to the model and from the model back to the data base. We begin with a simple example using the data tree in Figure III.B.I and then discuss some of the more general needs and philosophy of data transfer.

Suppose we wished to read into our model the values on the leftmost three terminal branches of the tree in Figure III.B.I and put the first value into a variable named $X$, the second into a variable named $Y$, and the third into a variable named $z$.

Our first step to perform the above task of course is to access the three values in order. Using the above procedure, we could first generate the position vectors IDV pointing to the three values we wish to access:

\begin{tabular}{l|c|c|c} 
& value & value & value \\
\hline $\operatorname{IDV}(3)$ & -0 & 0 & 0 \\
$\operatorname{IDV}(2)$ & 0 & 0 & 0 \\
$\operatorname{IDV}(1)$ & 1 & 2 & -3
\end{tabular}

For each of these three IDV vectors, we could then use the $n_{U}\left(j, k_{j}^{*}\right)$ functions (offsets) to access the corresponding value in the d-vector. Doing so would give us the values $d_{1}, d_{2}$, and $d_{3}$ we wish to place into $X, Y$, and $Z$ respectively. 
We now ask what is to be done with these values. The most naive approach would be to simply write these down and have our model read them. The data transfer system described here does not take this naive approach -- it transfers these values directly into the variables $X, Y$, and $Z$ using the idea of a "labelled common". Since "labelled common" is a FORTRAN-term, we will describe the way we accomplish this data transfer as if we had a FORTRAN program. However, the technique is quite general and could be applied using any computer language.

Returning to our problem, suppose we have accessed the three valuas $d_{1}, d_{2}$, and $d_{3}$ we need and placed them into an array $A$ of length 3. Suppose in the computer program where we performed this access, we had placed the FORTRAN statement.

\section{COMMON/XYZ/A(3).}

If in our model we merely place the FORTRAN statement

$$
\text { COMMON/XYZ/X,Y,Z }
$$

we will find that the value $d_{1}=A(1)$ appears inside the variable $X$, $d_{2}=A(2)$ appears inside the variable $Y$, and $d_{3}=A(3)$ appears inside the variable $Z$-- all without the necessity for any read or write instructions.

These simple ideas might motivate us to write a simple program we cal1 FILL which performs the following functions:

1. Loop through the IDV vectors that point to the needed data values. 
2. Access the terminal value corresponding to each IOV vector.

3. Place the terminal values as they are accessed into a single vector $A$.

Then by simply placing the statement

COMMON/XYZ/A (3)

into the FILL program and the statement

COMMON/XYZ/X,Y,Z

into our model, the data transfer will be fully automated, complete, and quite general.

The three stage procedure outlined above is conceptually quite simple but the first step, that of looping through all the required IDV vectors in the tree requires further explanation. In our simple model above, we must develop a method to loop through the three IDV vectors in which we are interested and to ignore the rest. Further, we must be certain that the three IDV vectors and the three variables $X, Y$, and $Z$ are properly paired -- otherwise we might place the value we wished to place in $X$ into $Y$ instead.

In order to facilitate this consistency, we have established a number of conventions for filling a labelled common. The first convention is to assume that all values that can be read into a particular labelled common must come from a single set. That is, two values from two distinct sets must be transferred into the model in two distinct labelled commons: This convention allows us to perform our FILL procedure at the set tree level rather than at the level of the full hierarchical data tree itself. The result of this assumption is to simplify our 
algorithm for looping over IDV settings to fill a labeled common. For the remainder of this discussion, when we say "tree" it will be understood that we are referring to a "set tree", not a full hierarchical tree.

Assuming that ourlabelled common is restricted to lie within a particular set, we will define two control variables for each labelled common. The first is a labelled common size vector and the second is a labelled common order vector. The positions in each of these two vectors correspond to dimensions in the set (i.e. levels in the set tree). The labelled common size vector allows the user to specify for each dimension how many values he would like that dimension to take. In our example above, he would have specified that the size of dimension 1 in labelled common /XYZ/ is 3 , indicating that he requires 3 terminal values for each setting of higher level branches. In that example, however, the user would wish to specify that the values at dimensions 3 and 2 are fixed. Thus he would place a 0 into the labelled common size vector in positions 3 and 2 . The labelled common size vector, which is user specified, would be

$$
\operatorname{LCS}=(3,0,0)
$$

This LCS specification tells our FILL procedure to generate the IDV vectors:

$\operatorname{IDV}(3)$

$\operatorname{IDV}(2)$
$\operatorname{IDV}(3)$

$\operatorname{IDV}(2)$

2
$\operatorname{IDV}(3)$

$\operatorname{IDV}(2)$ 
The IDV(-3) and IDV(2) settings are to be fixed and will be specified in a way to be made clear shortly.

The user must now specify in what order he wishes these IDV vectors to be generated so that he can place his variables $X, Y$, and $Z$ in the proper order. He does so by specifying a labelled common order vector. In that vector, the dimensions with positive sizes are ordered beginning with 1 -- the dimension indexed by a 1 will vary fastest, 2 second fastest, 3 third fastest,... when we generate the IDV vectors. In the simple example above, we require only a 1 in position 1.

The labelled common order vector is also used to tell us which dimensions (i.e. which settings of IDV) are fixed (i.e. are not allowed to change as we generate all the IDV vectors). This is accomplished by placing a negative number into each of the fixed set dimension positions in ascending order of dimension. To illustrate, the labelled common order vector for the simple example above would be

$$
L C O=(1,-2,-3),
$$

telling us to increment dimension 1 most rapidly and to hold dimensions 2 and 3 fixed.

To summarize our development thus far, the following procedure will transfer the necessary data into our model.

0 . Bring in IOV values for the dimensions that are fixed.

1. Loop through the IOV vectors that point to the needed data values. 
a. Establish upper limits on the free dimensions by reading the corresponding entry from the LCS vector.

b. Establish the order in which dimensions are to be incremented by reading the positive entries from the $L C O$ vector.

c. Establish which dimensions are fixed by reading the negative entries from the $\mathrm{LCO}$ vector.

d. Loop first over size by dimension and then over order by dimension, holding fixed dimensions constant, generating all IDV vectors.

2. Access the terminal value corresponding to each IDV vector.

3. Place the terminal values as they are accessed into a single vector $A$.

This is the essence of our FILL algorithm. The STOR algorithm is the inverse of this algorithm in the sense that steps 2 and 3 are replaced as follows.

1. Find the terminal branch corresponding to each IDV vector.

2. Place the next value from the vector $A$ into the storage postion corresponding to the terminal branch determined in 2 . 


\section{CHAPTER IV \\ USING THE COMPUTER MODELING SOFTNARE}

\section{A. USING THE EXECUTIVE SYSTEM \\ 1. Introduction}

The Executive serves as the interface between the user and the hierarchical data base. As discussed in Section II.B, the Executive contains a set of routines that allow the user to interactively insert, delete, change, and display values in the data base.

The Executive interacts directly with the tree through the creation of tables of data values. We call these tables containing model data hierarchical tables to distinguish them from system tables, which are. discussed in Section IV.A.2. The user specifies which hierarchical table he wishes to use to input data and output results by defining the system table called the Input/Output (IO) Table.

The dimensions of a hierarchical table must consist of a subset of the dimensions of the model. While hierarchical tables may contain any number of dimensions up to the number of dimensions in the model, the Executive is designed around the philosophy that the user can only conveniently work with two-dimensional tables. Thus the Executive, at any given moment, works with only a two-dimensional "slice" of an n-dimensional table. The user can modify n-dimensional tables by cycling through two-dimensional-slices until the table has been 
spanned. Certain Executive commands are designed to facilitate this cycling. The user specifies which two-dimensional slices of a hierarchical table he wishes to work with by specifying a single row in the 10 Table. Each row of the 10 Table defines a hierarchical table and sets the order in which the Executive will traverse that table using twodimensional slices.

Section IV.A.4 describes how to access the Executive. Section IV.A.2 describes briefly the five system tables which must be specified using the Executive. The two system tables which are needed to store data and names using the Executive are discussed in detail. action IV.A.3 contains a catalog of the commands that make up the Executive. They are described in the order in which they would normally be used. For each command the syntax, a description of the function and use of the command, and one or more examples are given.

2. System Tables

Using the Executive, five system tables must be specified:

LABELLED COMMON SIZE

LABELLED COMMON ORDER

STORAGE CONDITIONALITY

INPUT/OUTPUT

NAME CONDITIONALITY

The first three are used by the Data Transfer System when moviriy data between the hierarchical data tree and the labelled commons in the model logic. The LCS and LCO Tables are described in Section IV.B 
while storage conditionality is described in Chapters III and VI. As outlined above in Section II.B., the IO Table creates a mapping between hierarchical tables and the hierarchical data tree. It is used only in the Executive. It must be specified before the user may place any data or names in the tree. The NC Table specifies the storage of names in the tree. It is also used only in the Executive and must be specified before the user can place names in the tree.

Each system table has as many columns as there are model dimensions. The LCS and LCO Tables have as many rows as there are labelled commons, the SC Table has as many rows as there are data sets, the IO Table has as many rows as there are hierarchical tables defined by the user, and the NC Table has as many rows as there are model dimensions. All elements of all system tables must be integers, but the meaning of these elements varies from table to table.

\section{a. Input/Output Table}

The information required to define the mapping between the hierarchical data tree and the hierarchical tables is specified by the user and stored in the system table called the Input/Output (IO) Table. Each row in the in table defines a hierarchical table.

Since, the IO table contains as many columns as there are model dimensions, the definition of a hierarchical table requires one integer entry for each model dimension. A zero in the $i$ th position of a row in the 10 table indicates that the information in the hierarchical table defined by this row is independent of the ith dimension. A positive entry in the $i$ th position indicates that the data in this hierarchical 
table depend on the $i$ th dimension, but the $i$ th dimension is fixed at the value indicated. A negative entry in the $i$ th position indicates that the data in this hierarchical table vary over the $i$ th dimension. The dimension or "size" of a hierarchical table is given by the number. of negative entries in the row of the 10 table defining the hierarchical table.

For example, suppose rows 1 and 2 of the 10 table for a model with five dimensions are:

$$
\operatorname{Dim} 1 \quad \operatorname{Dim} 2 \cdot \operatorname{Dim} 3 . \operatorname{Dim} 4 \quad \operatorname{Dim} 5
$$

$\begin{array}{llllll}\text { 1. } & -2 & -1 & 0 & -3 & 1 \\ 2 . & -2 & -1 & 0 & -3 & 2\end{array}$

The data in Table 1 are independent of dimension 3. Those data do, however, depend on dimension 5, but for Table 1 the value of dimension 5 is fixed at 1. The data depend on and vary over dimensions 1,2 , and 4 , implying that Table 1 is a three-dimensional table.

Table 2 has the same characteristics as Table 1 except that the value of dimension 5 is fixed at 2 . Thus the data in both tables depend on the same dimensions $(1,2,4$, and 5$) ;$ i.e., all the data in these two tables are in the same data set. In this case the user could specify the I0 Table so that all the data are contained in a single hierarchical table. The I0 Table would be:

$\begin{array}{cccccc}\operatorname{Dim} 1 & \operatorname{Dim} 2 \cdots & \operatorname{Dim} 3 & \operatorname{Dim} 4 & \operatorname{Dim} 5 \\ 1.2 & -1 & 0 & -3 & -4 .\end{array}$

These examples are intended in part to illustrate how the user can work 
with either a few higher dimensional tables or many lower dimensional tables. No negative number may appear more than once in each row of the 10 Table. In general, negative numbers must be used in succession. The only exceptions to this are -1 and -2 , which are discussed below. When displaying a two-dimensional slice of a table, the dimension in which -1 appears varies over the rows; the dimension in which -2 appears varies over the columns. The name of the dimension in which -1 appears is the row heading; the name of the dimension in which -2 appears is the column heading. The transpose of a two-dimensional slice is obtained by interchanging the -1 and -2 .

For a particular two-dimensional slice, the dimensions in which higher negative numbers $(-3,-4, \ldots)$ appear are fixed; different values for these dimensions define different two-dimensional slices. The abbreviations of all the dimensions which are fixed for a particular two-dimensional slice, as well as the names of the values at which those dimensions are fixed, appear in the legend when a two-dimensional slice is displayed. If there is no -l entry in a row of the I0 Table, then the two-dimensional slices of the corresponding hierarchical table are row vectors. If there is no -2 entry, the slices are column vectors. If neither -1 nor -2 appears, the slices are individual data elements.

The user is faced with another trade-off here, this time between the number of two-dimensional slices and the number of elements in each slice. Some attention should be given to this issue as the IO Table is being defined.

A few. other rules for using the IO Table follow:

1.) As discussed in Chapter II, the attribute dimension is the 
lowest dimension, and all data depend on the attribute dimension. Thus the first entry in each row of the IO Table must be non-zero.

2) The rows of the IO Table may be ordered in any way the user chooses.

3) Negative entries other than -1 and -2 may not be skipped. For example, if a row of the 10 Table contains a -5 , then it must a) so contain $a-3$ and $a-4$.

4) Data from different data sets, i.e., data which depend on different dimensions, must be stored in different hierarchical tables since they require zeros in different positions in the rows of the 10 Table. This implies that to access all of the data for a model, there must be at least as many tables as there are data sets.

5) An individual data eiement may appear in more than one table, depending on how the user defines the tables. However, an individual data element will appear in only one set.

b. Name Conditionality

One of the five system tables is the Name Conditionality (NC)

Table. It must be specified by the user before any names may be placed in the data base. Its function is to reduce the amount of storage required for names and to reduce the time necessary for the user to specify names. It does this by making names conditional or unconditional on higher model dimensions. For example, suppose that one of the model dimensions is time, 
and that the model covers 10 time periods. If the same 10 periods are used in a.1 parts of the model, then. the names of the time periods are not conditional on the values of any other dimensions. Thus we need only store the names of the time periods once. Then whenever a table is specified with time as one of the dimensions of a two-dimensional slice, the names of the time periods will automatically be brought in from storage. This is much more storage efficient than to store the names individually with each two-dimensional slice. On the other hand, the names associated with some dimensions may be conditional on the values of other dimensions. For example, if the model dimensions were (1) attribute, (2) time, (3) activity, and (4) region, then the activity names might be conditional on which region we are referring to. This would occur, for example, if activity 1 in region 1 has a different name than activity 1 in region 2 . In this case the activity names would have to be specified once for each region. Suppose there are two tables in which activities vary over the rows, say activity by attribute and activity by time (for a. particular attribute). Then these activity names would have to be specified and stored once for each region, rather than twice, which would be required if there were no name conditionality.

Using name conditionality, names can be made conditional only on higher level dimensions. The user should consider this restriction when specifying his model dimensions. For the current example, we could make attribute (dimension 1). dependent on activity (dimension 2) andiregion (dimension 4), but we could not make region dependent on activity or attribute. If the 
user performs the mental exercise of analyzing the dimensional hierarchy from the standpoint of the names he wishes to use, he can often simplify both his dimensional assumptions and his naming conventions.

If the model data are arranged along NDIM dimensions, then the Name Conditionality Matrix is square with NDIM rows and NDIM columns. The rules for the matrix elements are as follows:

1) $a_{i j}=0 \quad$ if $i>j$

2) $a_{i j}=1 \quad$ if $i=j$

3) $a_{i j}=0 \quad$ if $i<j$ and names associated with particular values of dimension $i$ are independent of the value of dimension $j$.

4) $a_{i j}=1 \quad$ if $i<j$ and names associated with particular values of dimension $i$ are dependent on the value of dimension $j$.

For the current example, the user should specify the following Namie Conditionality Matrix:

\begin{tabular}{l|c|c|c|c|}
\multicolumn{1}{c}{ Attribute } & \multicolumn{1}{c}{$A T$} & \multicolumn{1}{c}{$T M$} & $A C$ & $R G$ \\
\cline { 2 - 5 } Time & 1 & $a_{12}$ & $a_{13}$ & $a_{14}$ \\
\hline \multirow{2}{*}{ Activity } & 0 & 1 & $a_{23}$ & $a_{24}$ \\
\cline { 2 - 5 } Region & 0 & 0 & 1 & $a_{34}$ \\
\hline & 0 & 0 & 0 & 1 \\
\hline
\end{tabular}

where $a_{i j}=0$ or 1 . 
To clarify our name conditionality conventions, we will consider the matrix one row, at a time. We assumed above that time is independent of the other dimensions. Thus $0^{\prime}$ 's should be placed in positions a 23 and a $24 \cdot$ We also assumed that the names associated with different values, of the activity dimension depend on the value of the region dimension. Thus a II should be placed in position a 34 . Finally, for the attribute row, we assume that. attribute names depend upon which activity and which region we are referring to, but not upon time. Thus we have $a_{12}=0$ and $a_{13}=a_{14}=1$. The final Name Conditionality Matrix is:

\begin{tabular}{l} 
Attribute \\
$\begin{array}{l}\text { Time } \\
\text { Activity } \\
\text { Region }\end{array}$ \\
\cline { 2 - 5 }
\end{tabular}$\quad$\begin{tabular}{ccc|c|c|}
\hline & AT & TM & AC & RG \\
\hline 0 & 0 & 1 & 1 \\
\hline 0 & 0 & 0 & 0 \\
\hline
\end{tabular}

The user specifies this table by "getting" the NC-system table, specifying the appropriate I's and O's, and "replacing" the NC system table., He, need not specify row and column names for the NC table, as these are automatically set to the dimension names and abbreviations which the user specified when he created the model. (See the following rataing of commands). 
3. Catalog of Executive Commands

Because of the detail in this section we have included an outline of the remainder of the section here. The Executive commands will be discussed in detajl in the followirig order:

a. Model Retrieval/Creation Command

i. GMOD

b. Workspace Manipulation Commands

i. $\quad$ SIZE

ii. INIT

iii. CHANGE

iv. $A D D$

V. DEL

vi. EXCH

c. Workspace Display Commands

i. SET

ii. DISP

d. System Table Commands

i. GSTB

ii. PSTB

iii. RSTB

e. Hierarchical Table Commands

i. GTAB

ii. NEXT

iii. $R T A B$

iv. PTAB

f. End of Session Command

i. END 
Explanation of Symbols

-User supplied value.

[] -optional.

\{\}

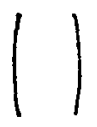

11

$\therefore$

- Only one of the enclosed options may be specified. Options are separated by semicolons. These semicolons are not part of the command line.

-Any number of the enclosed options may be specified. Options are separated by semicolons. These semicolons are not part of the command line.

-Denotes the message following is machine generated.

$4-11$ 
a. Model Retrieval/Creation Command 
The GMOD Command

GMOD, $<N>$

$\mathrm{N}>0$ and integer

Function: GMOD is an acronym for "Get Model". This command must be the first command issued in any session on the Executive. The GMOD command is used to gain access to the data of the model whose index is $N$. If such a model does not exist, then the user will be prompted for information necessary for the creation of a new model data base. The first prompt will request the model's name. If a carriage return is entered without giving a name then the new model data base will not be created and control returned to the user. The information necessary to create a model is the model name, the number of dimensions, and the names and abbreviations of the dimensions.

Example 1 - get model 5

GMOD, 5

- THE CURRENT MODEL IS DFI/DOE ENERGY MODEL

$\rightarrow \quad$ EXEC>

Example 2 - create model 6

GMOD, 6

$\rightarrow \quad$ A NEN MODEL WILL BE CREATED IF A NON-NULL STRING REPRESENTINC THE NEW MODEL'S NAME IS ENTERED.

NEW MODEL

$\rightarrow \quad$ ENTER THE NUMBER OF MODEL DIMENSIONS

1

$\rightarrow$ eNTER DIMENSION 1 NAME (MAXIMUM 20 CHARACTERS)

- ATTRIBUTE

$\rightarrow$ ENTER DIMENSION 1 ABBREVIATION. (MAXIMUM 4 CHARACTERS) AT

$\rightarrow \quad$ CURRENT MODEL IS NEW MODEL

$\rightarrow$ EXEC> 
b. Workspace Maniputation Comands 
The SIZE command

$S I Z E,\langle N 1\rangle,\langle N 2>$

$\mathrm{N} 1, \mathrm{~N} 2>0$ and integers.

$73+6(N 2+N 1)+(N 1 \times N 2) \leq 2000$

\section{Function:}

After a model has been retrieved or created by GMOD, the SIZE command is used to prepare an empty workspace called the Primary Area or Primary Table for one of two purposes. ("Workspace," "Primary Table," and "Primary Area" all refer to the same thing.) A new table or portion of a table may be created for placement in the model or an old table or portion of a table may be retrieved from storage for editing and replacement or simply to observe the values which reside there. The SIZE command sets the size of the workspace in terms of the number of rows and columns and places blanks in place of all names and tabie elements. The layout of the workspace is shown in Figure 1.

Example - set the size of the workspace to 35 rows by 11 columns SIZE, 35, 11

$+\quad$ EXEC> 


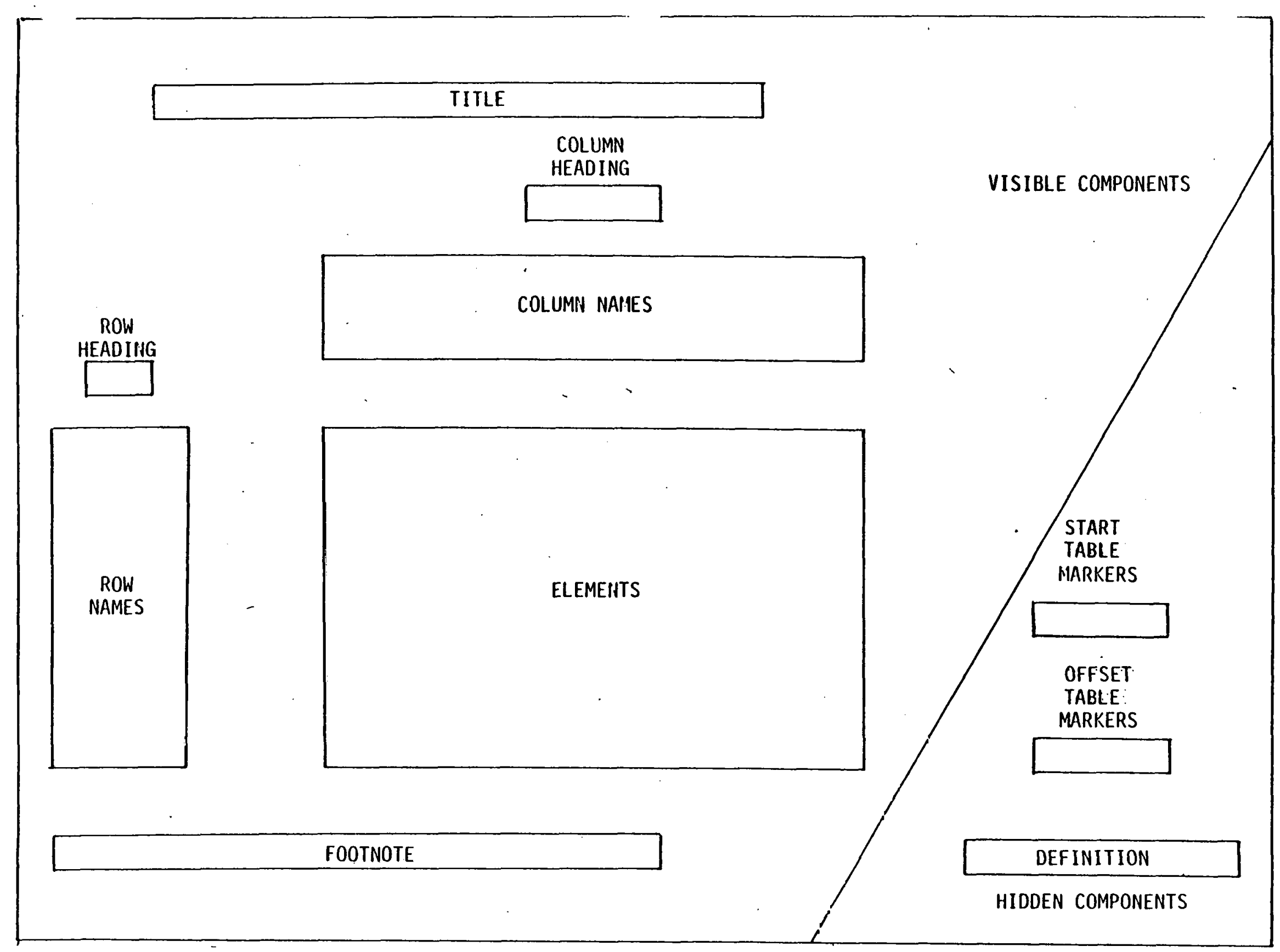

Figure IV.A, 1 THE PRIMARY AREA 
The INIT command

INIT $[$, <VALUE> $]$

$-1,000,000<$ VALUE $<1,000,000$

VALUE may be integer or real. If VALUE is real than all numbers between $-.0001<$ VALUE< .0001 are treated as zero.

Function: The INIT command is used to eliminate any information in the workspace or to initialize all elements in the workspace to an initial value. If VALUE is specified then all names, headings, the title and the footnote are set to blanks and the table elements are set to VALUE. If VALUE is not specified then the table elements are also blanked. It should be noted that blanks are not equivalent to zero:

Example 1 - blank out everything

\section{INIT}

$\rightarrow \quad$ EXEC>

Example 2 - initialize all elements to 5.301

INIT , 5.301

$\rightarrow$ EXEC> 
The CHANGE Command

There are four basic ways to use the change command which will be discussed separately. The parameters of the command and their usage are similar in each case. The CHANGE command is used to modify existing tables and also to create new ones by replacing blanks in the workspace with elements and names, and then storing a copy of the workspace. The CHANGE command is used for modifying and creating both system tables and hierarchical tables.

\section{A. Element}

$$
C, E,\langle I\rangle,\langle J\rangle,\langle\text { VALUE }\rangle
$$

$1 \leq I \leq$ number of rows in the workspace

$1 \leq \mathrm{J} \leq$ number of columns in the workspace

VALUE has the same restrictions as for the INIT command.

Function: With an $E$ as the second parameter the change command will change a particular element of the workspace defined by the values I and 了.

Example - change element $(1,3)$ to 1.00

$C, E, 1,3,1.00$ 
B. Row or Column

$$
c,\langle\text { PART }\rangle,\left\langle I=[,\langle J\rangle] / v_{1}\left[\ldots, v_{n}\right]\right.
$$

PART $={ }^{-} \varepsilon\{R, C\}$

$1 \leq \mathrm{I} \leq$ number of rows in the primary table.

$1 \leq J \leq$ number of columns in the primary table.

$J \leq J+n-1 \leq$ number of columns in the primary table

(if J specified)

$1 \leq n \leq$ number of columins in the primary table

(if $\mathrm{J}$ specified)

All $V_{i}$ 's have the same restriction as VALUE in the INIT command

Function: The change command will change an entire row or column or a portion of a row or column depending on whether the second parameter is an $R$ or a $C$ and on how many $V_{i}$ 's are specified. The change command will change the values of the elements in row (column) I starting with the element in column (row) $J$ to the values $v_{1}, \ldots, v_{n}$ where any of the $v_{i}, i=, \ldots, n$ can be of type real or integer. If $J$ is not specified, the change starts with the first element in the row (columin). The change continues until the $V_{i}$ 's are exhausted. If more $V_{j}$ 's are specified than will fit into the primary area, the command fits in as many as possible and generates a diagnostic.

Example - change elements 5 through 8 of row 2 $C, R, 2,5 / 2.0,1.0,7,999$.

$\rightarrow \quad$ EXEC> 
C. Row Name or Col umn Name

$$
C,\left\langle\text { PART }>,\langle I\rangle^{\prime},\left\langle T_{E X T} \text { STRING }_{1}>\left[, \ldots,<\text { TEXT STRING }_{n}>\right]\right.\right.
$$

PART $\varepsilon\{R N, C N\}$

$I \leq I \leq$ number of rows in the primary table

$I \leq n+I-I \leq$ number of rows in the primary table.

Text string 1 is of the form:

$$
\text { <DELIMITER> <STRIMG> <DELIMITER> }
$$

where STRING is a string of less than 25 valid characters and <DELIMITER> is any valid character not appearing in STRING. (A1l characters other than control characters and asterisk (*) are valid.)

Function: With $\mathrm{RN}$ or $\mathrm{CN}$ as the second parameter of the change command the user can change row names or column names, respectively. The change command will change the row (column) names in the workspace, starting with row (column) I, to the values TEXT STRING,$\ldots$, TEXT STRING ${ }_{n}$. Continue until either the text strings are exhausted or the last row (column) name is changed. If more text strings exist than will fit in the workspace, change as many as possible and generate a diagnostic. A column name is printed as three groups of eight characters each, so that appropriate blanks must be left in TEXT STRING when entering column names.

Example - change the name of row 4 to PRICE

C, RN, 4, \$PRICE\$

$\rightarrow$ EXEC>. 
D. Title or Footnote

$$
C,\langle P A R T\rangle,\langle T E X T \text { STRING }
$$

PARTE $\{T L, F N\}$

TEXT STRING is as in part $C$, except that string may have up to 60 characters.

Function: With $T L$ or FN as the second parameter the change command will change the title (TL) or the footnote (FN) of the workspace table to TEXT STRING. (When a title or footnote is placed in the data base as part of a particular two-dimensional slice of a table, it becomes part of each two-dimensional slice of that table. Different slices of the same table may not have different names and footnotes.) 
The ADD Command

$$
\begin{aligned}
& \text { ADD, }\langle P A R T\rangle,\langle N 1\rangle,\langle\langle N 2>] \\
& \text { PART } \varepsilon^{\prime}\{R, C\} \\
& N 2 \geq N 1>0 \\
& N 1, N 2 \text {, are integers. }
\end{aligned}
$$

Function: The ADD command is used to add one or more columns or rows to the workspace table. PART may be either $R$ or $C$ as in the EXCH command. $\mathrm{N} 1$ is the row or column number just after the place where the insertion is to be made. If $\mathrm{N} 2$ is used, it represents the last row or column inserted where $(N 2-N 1+I)$ is equal to the number of rows or columns inserted. The ADO command will add the rows or columns in the appropriate place and renumber the remaining rows or columns. The new rows/columns will be blank.

Example - add 3 rows just before row, 5. What was row 5 will become row 8 . $A D D, R, 5,7$

$\rightarrow \quad$ EXEC> 


\section{The OEL Command}

$D E L,\langle P A R T\rangle,\langle N 1\rangle,[\langle N 2\rangle]$

PARTE $\{R, C\}$

$\mathrm{N} 2 \geq \mathrm{N} 1>0$

N1,N2 are integers

Function: DEL is an acronym for "Delete". The DEL command is used to delete rows or columns. PART may be $\mathrm{R}$ or $\mathrm{C}$ as in the EXCH command. If $\mathrm{NI}$ alone is used then that row or column is deleted. If $N_{2}$ is specified then all rows or columns from $\mathrm{N} 1$ through $\mathrm{N} 2$ are deleted. Remaining rows or columns will be renumbered. (See the note under the RTAB command for further information on deleting rows or columns from already-existing tables.)

Example - delete rows 1 through 3. What was row 4 will become row 1 . $D E L, R, 1,3$

$\rightarrow \quad$ EXEC> 
The EXCH Command

EXCH, <PART>, <N1>, <N2>

PARTE $\{R, C\}$

$\mathrm{N} 2 \geq N 1$

N1, N2 are integers

Function: EXCH is an acronym for "Exchange". The EXCH command is used to exchange any two rows or any two columns. PART may be either $R$ or $C$ for rows or columns. Names are exchanged as well as values.

Example - exchange rows 3 and 6

EXCH, R, 3,6

$\rightarrow \quad$ EXEC> 
1

c. Workspace Display Commands. 
The SET command

SET, $\angle$ PARAMETER $>\left[, \ldots, \quad<\right.$ PARAMETER $\left._{n}>\right]$

PARAMETER $_{i}=\{$ COLUNINS $=\langle N\}\rangle$

$$
\begin{aligned}
& \text { LINES }=\langle N 2\rangle, \\
& \text { FORMAT }=\langle M\rangle, \\
& \text { SUPPRESS }=\langle\text { SWITCH }\rangle\}
\end{aligned}
$$

$N 1, N 2>0$ and integers

$45<\mathrm{N} 1 \quad 16<\mathrm{N} 2$

:? $\varepsilon\{F, E, G\}$

SWITCH $E\{O N, O F F\}$

Function The SET command is used to set parameters for outputting tables. All of these parameters have been assigned default values at logon time, so it is not necessary to give a "SET" command before outputting tables. N1 specifies the width of the output page and N2 its length. Their default values are 72 and 50 , respectively. $M$ specifies the type of format to use when outputting real valves. The resulting format will be $\langle M\rangle 9.2$, and the default is $F$. If SWITCH is ON the blank values will not be considered a part of the tabie. In this case, if a row (column) exists such that it and all rows (columns) greater than it contain no non-blank values, then the row (column) will not be printed. If the default switch is OFF, then the entire workspace will be printed, regardless of blanks. The default for the switch is $O N$.

Example - set page width to 80 columns

SET, WIDTH $=80$

$\rightarrow$ EXEC> 
The DISP Command

$\operatorname{DISP}[,<N 1>[,<N 2>[,<N 3>[,<N 4>]]]\}$

$N 2 \geq N 1>0$

$N 4 \geq N 3>0$

$\mathrm{N} 1, \mathrm{~N} 2, \mathrm{~N} 3, \mathrm{~N} 4$ are integers

Function: DISP is an acronym for "Display". The DISP command is used to display the current contents of the workspace. The parameters refer to the rows and columns of the workspace, not the rows and columns of the hierarchical table from which the data in the workspace were taken. (If only part of a hierarchical table was placed in the workspace, the numbering of rows and columns may differ between the table and the workspace.) Rows N1 through N2 and columns N3 through N4 are displayed. If only $N 1$ is specified then all rows starting with that row are retrieved. In order to specify $N i, N 1$ through $N(i-1)$ must be specified.

Example 1 - get Table 3 and display it

- GTAB, $3^{-}$(this command is explained later.)

$\rightarrow \quad E X E C>\quad-$

DISP

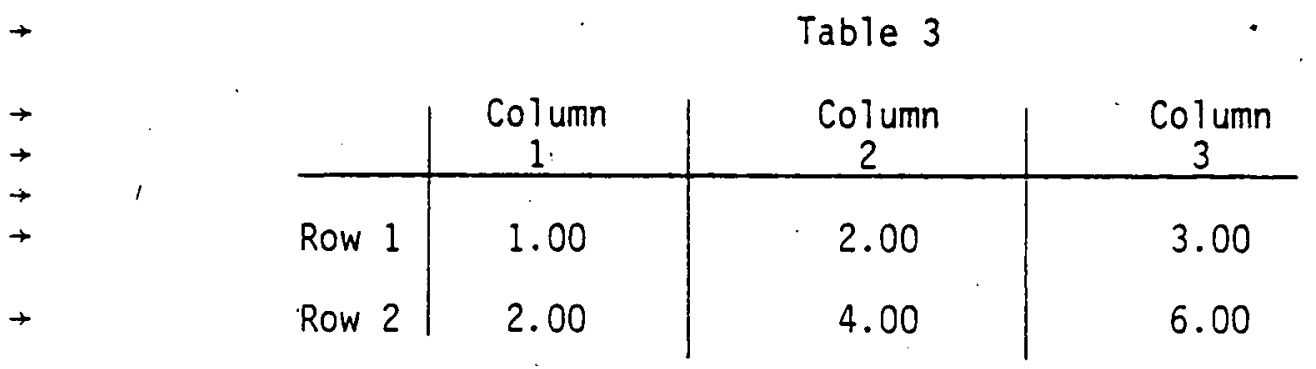

$\rightarrow \quad$ EXEC 
Example 2 - get table 4 and display rows 2 through 5, columns 3 and 4

$$
\begin{aligned}
& \text { GRAB, } 4 \\
&+\quad \text { EXEC> } \\
& \text { ISP, } 2,5,3,4
\end{aligned}
$$

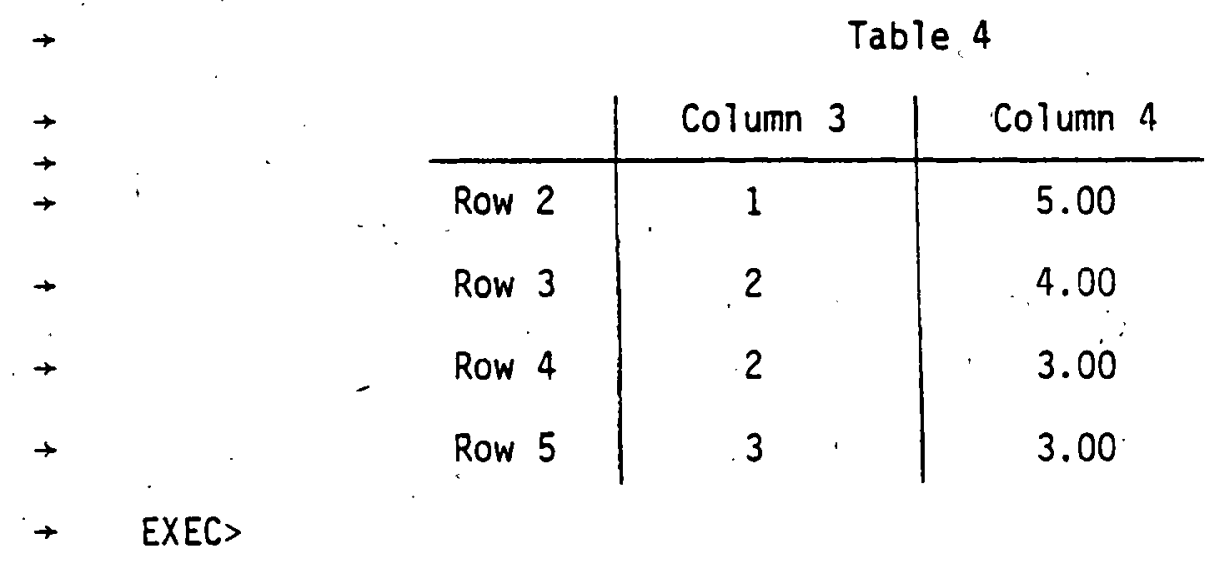

$4-28$ 
d. System Table Commands

$\checkmark$ 
The GSTB command

GSTB, <TYPE $>[,\langle N i>[,\langle N 2\rangle]]\{[, E][, N]\}$

TYPE $=\quad$ IO for Input/Output Table

LCS for Labeled Common Size Table

LCO for Labeled Cormon Order Table

NC for Name Conditionality Table

SC for Storage Conditionality Table

$\mathrm{N} 2 \geq \mathrm{N} 1>0$ and integers.

Function: GSTB is an àcronym for "Get System Table." System Tables are the Input/Output Table, Labeled Common Size Table; Labeled Common Order Table, Name Conditionality Table and Storage Conditionality Table. The use and format of the system tables is described in Section IV.A.3. The GSTB command will get a system table or a portion of a system table and place it in the workspace. The type of system table is determined by the TYPE parameter. N1 specifies the first row to be returned and N2 specifies the last row. If only $N 1$ is specified then only that row will be retrieved. If neither $N 1$ or $N 2$ is specified, then retrieval will start with the first row and continue until either all rows of the desired TYPE have been retrieved or the workspace is filled. In all cases, the first row retrieved will be placed in the first row of the primary table. If $E$ is specified, then only entries will be retrieved. If $N$ is specified, then only names will be retrieved. If neither $E$ nor $N$ is specified then both names and entries will be retrieved. 
Example 1 - retrieve rows 1-10 of the Input/Output Table

GSTB $, 10,1,10$

$\rightarrow$ EXEC>

Example 2 - retrieve the entire Labeled Common Order Table GSTB, LCO

$\rightarrow \quad$ EXEC $>$

Example 3 - retrieve the names for the Input/Output Table GSTB, $10, \%$

$\rightarrow \quad$ EXEC> 
The RSTB command

RSTB, <TYPE $[\langle N 1\rangle[,\langle N 2\rangle]]^{\prime}\{[, E][, N]\}$

TYPE = I0 for Input/Output Table

LCS for Labeled Common Size Table

LCO for Labeled Common Order Table

NC for Name Conditionality Table

SC for Storage Conditionality Table

\section{$\mathrm{N} 2 \geq \mathrm{N} 1>0$ and integers}

Function: RSTB is an acronym for "Replace System Table". "The RSTB command is used to replace data currently residing in the workspace and corresponding to a system table in the data base. The type of the system table is determined by the TYPE parameter. N1 specifies the row in the system table where the first row of the workspace is to be placed and N2 specifies the last row of the workspace to be placed in the system table. The first row to be placed in the system table corresponds to the first. row of the workspace. If only $\mathrm{Nl}$ is specified, the first row of the workspace will be placed in the row of the system table specified by N1. If neither. $\mathrm{N} 1$ nor $\mathrm{N} 2$ is specified and the data currently in the workspace was retrieved via a GSTB command then the first row of the workspace will be replaced in the same position from which it was retrieved, the second row in the same position from which it was retrieved and so on until the last row to contain a non-BLANK value is replaced. The $E$ and $N$ options are used to replace only the elements or names in the table as desired. ilames associated with rows or columns beyond the last blank row or column are ignored. 
Example 1 - Change the third row of the IO Table. Assume there are 5 model dimensions

SIZE, 1,5

$$
\begin{array}{ll}
\rightarrow & \text { EXEC }> \\
& C, R, 3 /-1,0,0,-2,1 \\
\rightarrow \quad & \text { EXEC> } \\
& \text { RSTB }, 10,3 \\
+\quad & \text { EXEC> }
\end{array}
$$

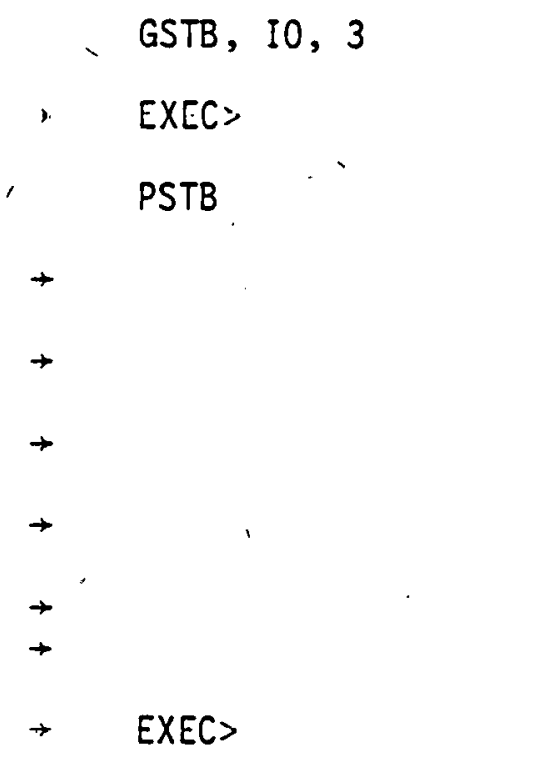

INPUT/OUTPUT

AT TM KN SC LR

i $2 \begin{array}{llll}2 & 3 & 4 & 5\end{array}$

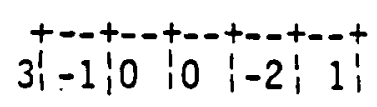

4-34 
Jr row 3 in the previous example

\section{IMETERS \$}

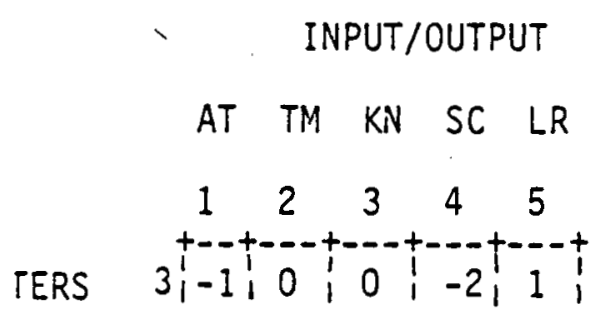


e. Hierarchical Table Commands 
The GTAB Command

$$
\begin{aligned}
\text { GTAB, } & <N>[/<D 3>[, \ldots,<D L>]][/<N]>[,\langle N 2>[,<N 3>[,<N 4>]]]] \\
& \{[, E] ;[, R N] ;[, C N]\}
\end{aligned}
$$

$-i t>0$

$N 2 \geq i l 1>0$

$\mathrm{N} 4 \geq \mathrm{N} 3>0$

$D 3, \ldots, O L>0$

$L<M$

$\mathrm{N}, \mathrm{N1}, \mathrm{N2}, \mathrm{N} 3, \mathrm{~N} 4, \mathrm{D3}, \ldots \mathrm{DL}$ are integers

$M=$ absolute value of most negative entry in row $N$ of the Input/ Output Table.

Function: GTAB is an acronym for "Get Table". The GTAB command is used to place a copy of a particular two-dimensional slice of Table is into the workspace. IN is the index of the row, of the Input/Output Table which defines the table to be retrieved. D3 through DL may be used to specify which two-dimensional slice of a multi-dimensional table the user wishes to place in the workspace. They specify the values of the dimensions in which -3 through $-L$ appear in row $N$ of the Input/Output Table. Varying dimensions not specified (those from $-(L+1)$ through $-M$ ) are automatically set to 1. Rows N1 through N2 and columns N3 through N4 are retrieved and placed in the workspace. If none of the $\mathrm{Ni}$ 's are specified then the entire two-dimensional slice is retrieved (subject to size of the primary area). If only $N I$ is specified then all rows starting with that row are retrieved. In order to specify $\mathrm{Ni}$, NI through 
' $N(i-1)$ must be specified. $E, R N$, and $C N$ can be used to bring in any combination of elements, row names, and column names. All three are brought in if none is specified.

Example 1 - initialize a table to contain all ones and have the same row names as Table 5 and the same column names as Table 7. (If Table 5 or Table 7 has more than two dimensions, this gets the names from the first two-dimensional slice.)

SIZE, 10,10

$\rightarrow \quad$ EXEC>

INIT, 1.00

$\rightarrow$ EXEC>

GTAB, 5: 1,10 , RN

$\rightarrow \quad$ EXEC>

GTAB, $7: 1,1,1,10, \mathrm{CN}$

$\rightarrow \quad$ EXEC>

Example 2 - place rows 1-4 and columns 1-6 of Table 2 (or the first twodimensional slice of Table 2 if it has more than two dimensions) in to the workspace.

SIZE, 10,10

$\rightarrow \quad$ EXEC>

GTAB, 2: 1, 4, 1, 6

$\rightarrow$ EXEC? 
Example 3 - place the row names and elements of the two-dimensional

slice of Table 4 for which the value of the third fastest

varying dimension is 2 and the value of the fourth fastest varying dimension is 3 into the workspace

GTAB, 4/2, 3/ RN, E

$\rightarrow \quad$ EXEC> 
The NEXT Command

NEXT $[, U P][,<N]>[,<N 2>[,<N 3>[<H 4>]]]]([, E] ;[, R N] ;[, C N])$

$\mathrm{N} 2 \geq \mathrm{N} 1>0$

$N 4 \geq N 3>0$

$N 1, N 2, N 3, N 4$ are integers

Function: The NEXT command is used in conjunction with the GTAB command to provide an automated way of calling successive two-dimensional slices of a multi-dimensional table. If a table is defined as two dimenstonal in the IO Table then GTAB is all that is required to retrieve it. If . a table is defined as three or more dimensions then the GTAB command gets only the two-dimensional slice specified by the user (see GTAB command). By using a series of NEXT and NEXT,UP commands all succeeding two-dimensional slices of thic table may be retrieved.

Giving a NEXT command increments the value of the dimension in which -3 appears in the IO Table by 1 and places the corresponding twodimensional slice in the workspace. Giving a NEXT,UP command after NEXT command or GTAB command increments the value of the dimension in which -4 appears in the 10 Table by 1 , resets the value of the -3 dimension to 1 , and places the corresponding two-dimensional slice in the workspace. If M successive NEXT, UP commands are given after a NEXT command or GTAB command, the value of the dimension in which $-(M+2)$ appears in the IO Table is incremented by 1 , the values of the -3 through $-(M+1)$ dimensions are reset to 1 , and the corresponding two-dimensional slice is placed in the workspace. 'If there is no dimension in which $-(M+2)$ appears, then the Executive assumes the table is complete. The other parameters are used exactly as in GTAB. 
Example

Table 2 is defined in the $1 / 0$ system Table as

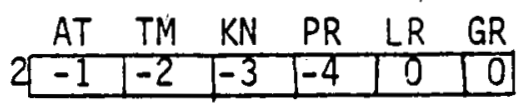

Retrieve Table 2.

GTAB , 2

$\rightarrow \quad$ EXEC>

NEXT

$\rightarrow \quad$ EXEC>

NEXT

$\rightarrow \quad$ EXEC>

NEXT, UP

$\rightarrow \quad$ EXEC>

NEXT

$\rightarrow \quad$ EXEC>

NEXT

$\rightarrow \quad$ EXEC>
Definition of Two-Dimensional

Slice Currently in workspace

\begin{tabular}{|l|l|l|l|l|l|}
\hline 1 & -2 & 1 & 1 & 0 & 0 \\
\hline
\end{tabular}

\begin{tabular}{|l|l|l|l|l|l|}
\hline-1 & -2 & 2 & 1 & 0 & 0 \\
\hline
\end{tabular}

\begin{tabular}{|l|l|l|l|l|l|}
\hline-1 & -2 & 3 & 1 & 0 & 0 \\
\hline
\end{tabular}

\begin{tabular}{|l|l|l|l|l|l|}
\hline-1 & -2 & 1 & 2 & 0 & 0 \\
\hline
\end{tabular}

\begin{tabular}{|l|l|l|l|l|l|}
\hline-1 & -2 & 2 & 2 & 0 & 0 \\
\hline
\end{tabular} 
NEXT, UP

$\rightarrow \quad$ EXEC>

NEXT

$\rightarrow \quad$ EXEC>

NEXT

$\rightarrow$ EXEC>

NEXT,UP

$\rightarrow$ EXEC>

NEXT,UP

- 'THe table has been completed

$\rightarrow \quad$ EXEC> \begin{tabular}{|l|l|l|l|l|l|}
\hline-1 & -2 & 1 & 3 & 0 & 0 \\
\hline
\end{tabular}

\begin{tabular}{|l|l|l|l|l|l|}
\hline-1 & -2 & 2 & 3 & 0 & 0 \\
\hline
\end{tabular}

\begin{tabular}{l|l|l|l|l|l|}
\hline-1 & -2 & 3 & 3 & 0 & 0 \\
\hline
\end{tabular} 
The RTAB Command

$\operatorname{RTAB}[,\langle\mathrm{N} 1\rangle[,\langle\mathrm{N} 2\rangle[,\langle\mathrm{N} 3\rangle[,\langle\mathrm{N} 4\rangle]]]]([, \mathrm{E}] ;[, \mathrm{RN}] ;[, \mathrm{CN}])$

$\mathrm{N} 2 \geq \mathrm{N} 1>0$

$\mathrm{N} 4 \geq \mathrm{N} 3>0$

$\mathrm{N1}, \mathrm{N2}, \mathrm{N3}, \mathrm{N4}$ are integers

Function: RTAB is an acronym for "Replace Table". The RTAB command is used to place a copy of the workspace into the table which was defined by the most recent call to GTAB. Thus if a table was previously retrieved by GTAB, $5,1,4,1,6$, then the command RTAB with no options would replace it in the same place in the OBSS. If it is desired to place the workspace in a different section of the same table, then the parameters $N i$ through N4 are used in the same way as in GTAB. In addition, E, RN and CiN can be used to replace any combination of row names, column names or elements. If none of the three is specified, all three are replaced.

Example 1 - get table 4 and replace it without change GTAB , 4

$\rightarrow \quad$ EXEC>

RTAB

$\rightarrow \quad$ EXEC> 
Example 2 - get table 4 rows $1-2$ and columns 1-5 and replace the elements only into Table 4 rows $5-6$ and columns 1-5.

GTAB $, 4,1,4,1,5$

$\rightarrow \quad$ EXEC>

$\operatorname{RTAB}, 5,6,1,5, E$

$\rightarrow \quad$ EXEC>

Note: If none of the $\mathrm{Ni}$ 's are specified then only the non-blank parts of the workspace are placed in the data base. This information is particularly useful when the user wishes to delete values or names from the data base. He must then use the $\mathrm{Ni}$ 's to ensure that unwanted parts of the data base are replaced with blanks.

Example 3 - get table 3 , which contains 4 rows and 3 columns, delete the last column, and replace the table:

GTAB, 3

$\rightarrow \quad$ EXEC>

OEL , $C, 3$

$\rightarrow \quad$ EXEC> .

$\mathrm{RTAB}, 1,4,1,3$

$\rightarrow \quad$ EXEC>

If the parameters after the RTAB had not been specified, only the non-blank parts of the workspace (i.e., the first two columns) would have been replaced, and the third column would remain in the data base. 


\section{The PTAB Command}

$P T A B,\langle N>[, O F F]$

$N$ is integer

Function: PTAB is an acronym for "Print Table", the PTAB command is used to print the contents of the table defined by the $N^{\text {th }}$ row of the Input/Output table. Unlike DISP it can be used to print tables of more than two dimensions. For example, if Table 3 were four dimensional then the PTAB command would successively get and print as many two dimensional slices of that table as necessary to represent it. Thus this command is equivalent to a series of NEXT; NEXT,UP; and DISP commands repeated for each two dimensional slice. The OFF option is used to print the tables offline.

Example - print table 6 offline

PTAB, 6, OFF

+ EXEC> 



\section{The END command}

END

Function The END command is used to end a session on the CMS Executive. New data entered or edited has al ready been placed in the Data Base Storage System by the RSTB and RTAB commands so there is no SAVE command per se. After the END command is given control is returned to the operating system where the user may run the model if desired. 


\section{Accessing the Executive}

To run the EXECUTIVE set up the following CLIST and execute it.

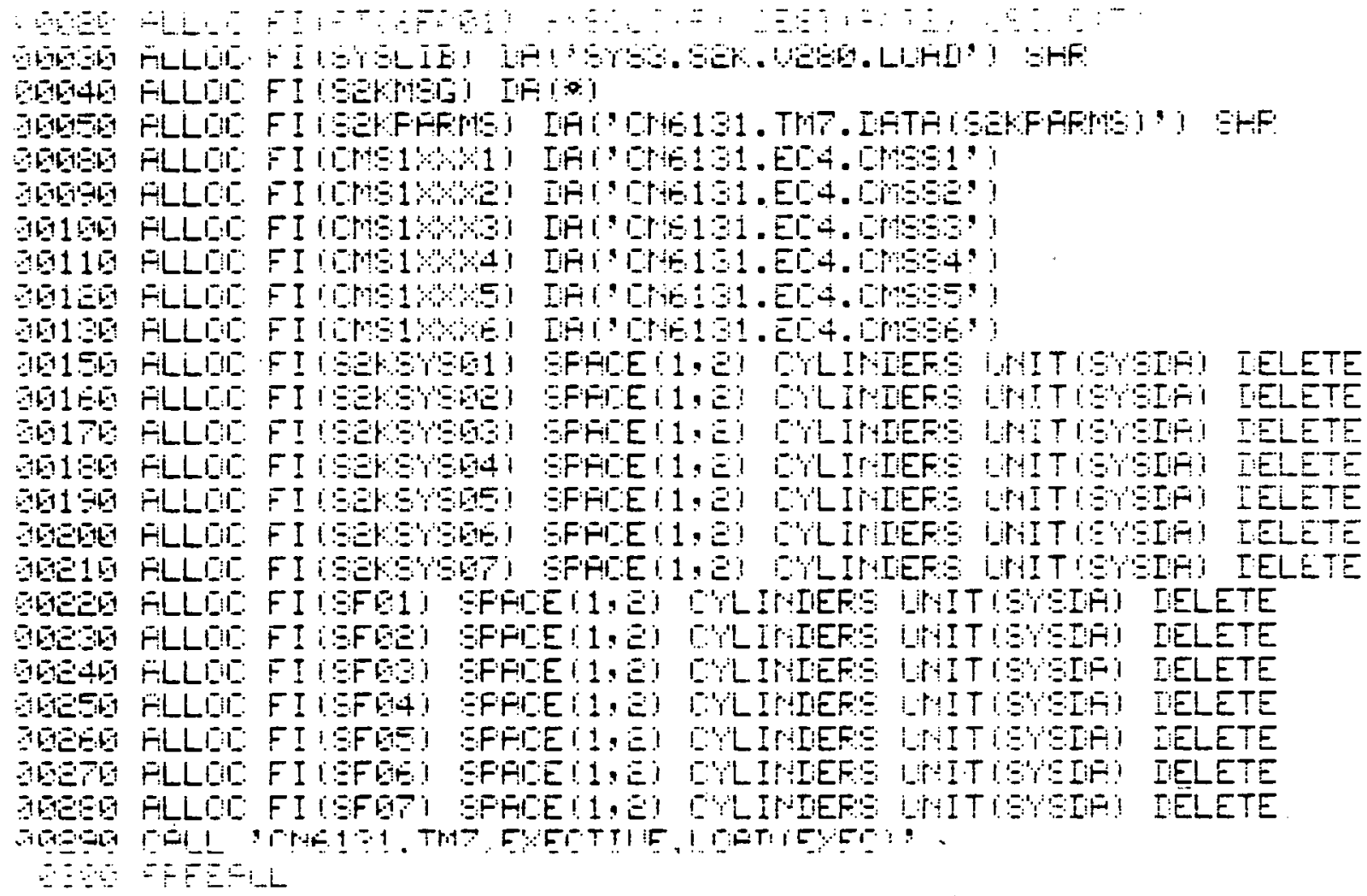

If a data base other than CN6131.TM1. CMSS is to be accessed, substitute the appropriate name into lines 80 through 130 above before executing. 
B. DATA TRANSFER SYSTEPY USE

This section provides a user's guide to the CMS Data Transfer System. It is addressed to the reader who has either designed or built a FORTRAN model and is now asking the question: How do I interface my model with the Data Transfer System? As such, it assumes the reader is familiar with the concepts developed in Sections II.A and. II.C. Further, it does not deal with the algorithms or inner workings of the system, which are covered in Chapter VI.

. On the highest level, the data transfer system consists of six FORTRAN subroutines: SOFTIN, SOFTOU, FILL; STOR, SFILL and SSTOR. By appropriate calls to these routines, the modeler controls the transfer of data to and from the labelled common areas in his model. To interface a model with the data transfer system, the user. must perform 4 tasks:

1. Define labelled conmons organized along dimensional lines.

2. Store information about the structure of each labelled common in the Labelled Common Size and Labelled Common Order Tables.

3. Design appropriate calls to routines FILL, SFILL, STOR, and SSTOR.

4. Construct a main program to call routines SOFTIN and SOFTOU. The following five subsections are arranged in this order with each subsection addressing a specific task. Going through them in order will provide a guide to preparing a data transfer system interface for any program.

1. Labelled Common Design. 
In FORTRAN, the COMMON statement is used to cause the sharing of storage by two or more program units and to specify the names of the variables and arrays that are to occupy this area. In other words, labelled common areas supply a means of communication between subroutines. The data transfer sy'stem also uses the labelled commons as a means of organizing the data in a model along dimensional lines.

The IBM FORTRAN labelled common statement is of the form:

$$
\text { COMMON } / r / a_{1}\left(k_{1}\right), a_{2}\left(k_{2}\right), \ldots
$$

where:

Each a is a variable name or array name.

Each $k$ (optional) represents the size of the array

Each $\underline{r}$ represents a name consisting of one through six alphanumeric characters, the first of which is alphabetic.

Labelled commons with the same name address the same area in memory. Thus, assume the main program of a model had the following common statement:

COMMON/DATA/ $X, Y, Z(100)$

with $X, Y$, and $Z$ of type $R E A L * 4$. This statement defines a common area named "DATA" with 408 storage locations ( 4 for each variable). Assume that subroutine' $G$ called from the main program had the statement:

COMMON/DATA/ $B(2), C(50), D(50)$

with $B, C$, and $D$ of type $R E A L * 4$. These variables now address the same storage locations as $X, Y$, and $Z$ in the main program. Assume that Subroutine $H$ is called from subroutine $G$ and contains the statement:

COMMON/DATA/ $K(3), L$ 
where $K$ is type REAL*4. K now addresses the same storage locations as the above variables with the correspondences shown in Table IV.B.1.

The data transfer system requires that the user define his labelled commons along clear dimensional lines. In particular, each labelled common must be defined to access a given data set or a well dfined subset of a given data set. In the second case, the modeler will generally define his labelled commons in such a fashion so that as he executes various subprograms he can sequentially access different subsets of the same Jata set as needed.

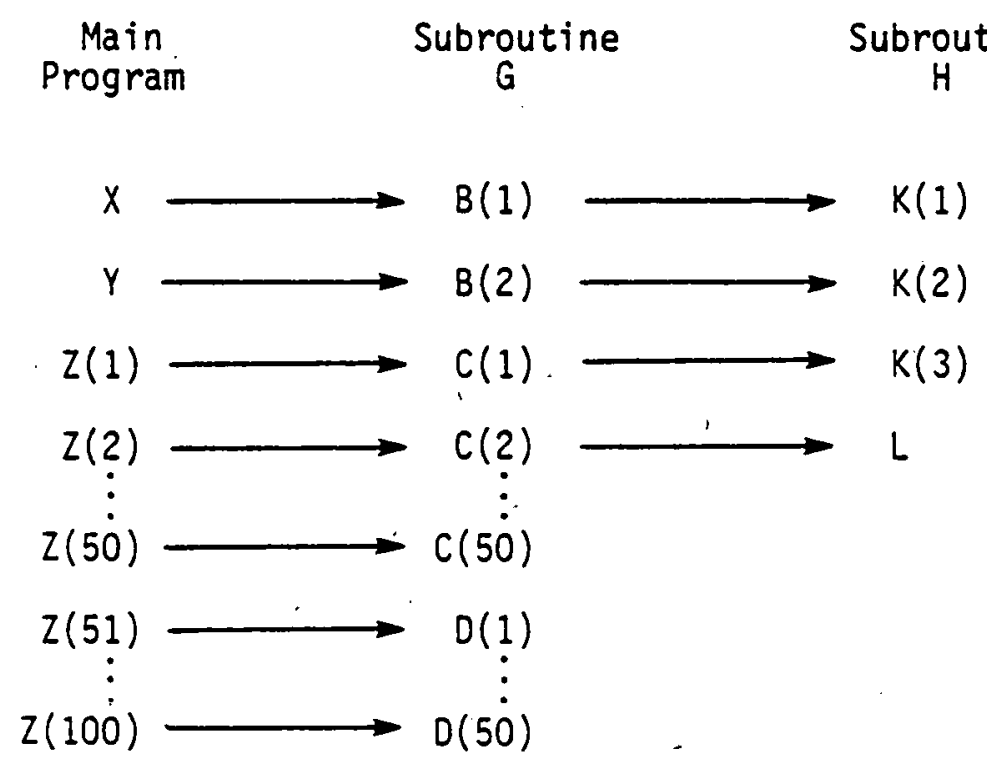

Table IV.B.1: LABELLED COMMON CORRESPONDENCES 
To illustrate this principle, consider the present value example of Chapter II disaggregated over two different cash flow streams representing two plants. Now, the model we wish to solve is given by the equations:

$$
P V_{i}=\sum_{t=1}^{N} c f_{i t}\left(\frac{1}{1+r}\right)^{t}
$$

where:

$P V_{i}=$ present value of the cash flow for the $i^{\text {th }}$ plant. $\quad(i=1,2)$

$N=$ number of time periods

$c f_{i t}=$ cash flow forplant $i$ in period $t(i=1,2 ; t=1, N)$

$r=$ discount rate

The logical way of extending. this example to this level of disaggregation would be to add a third dimension: plant. This new dimension would result in another level of the hierarchical tree as shown in Figure IV.B.1 This tree is a direct extension of the one in Figure II.A.2.

Suppose the user defined the following labelled common statements: COMMON/GLOB1/N, R.

COMMON/PL2/PV

COMMON/PLTI3/CF (50)

Notice that each labelled common contains data from only one set. Common GLOBI contains all values in the model which are independent of

This tree assumes that the new dimension (plant) is added as the highest level. It could have just as easily become the second lever. 
Plant

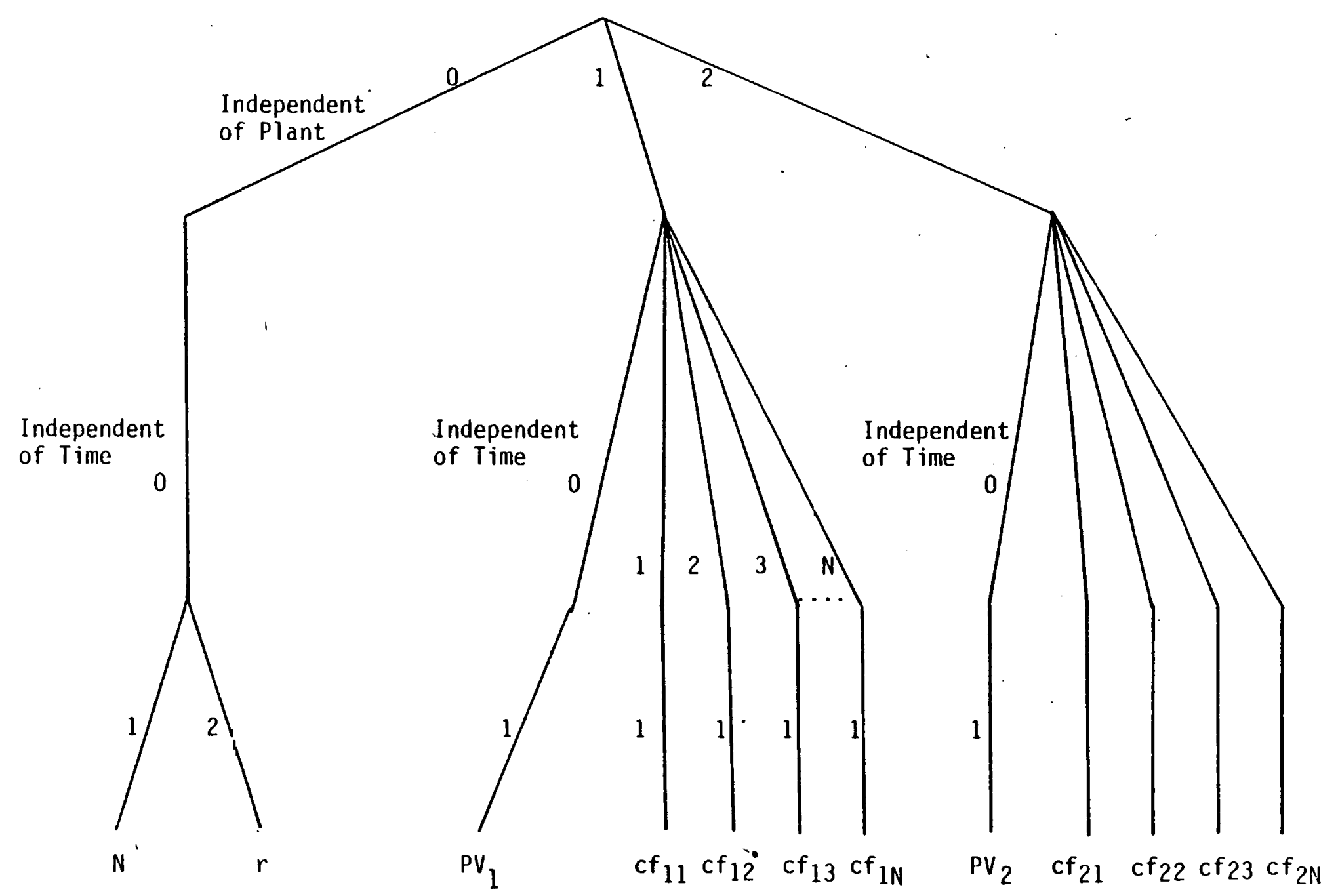

Figure IV.B.1 HIERARCHICAL TREE FOR EXAMPLE 
both time and plant. Common PL2 contains a value in the model which is independent of time for a given plant. An outline for a FORTRAN routine to run this model is given below:

1. Fill common GLOB1 with vaiues of $N$ and $R$

2. Loop over plants; $I=1,2$

3. Fill common PLTI3 with values of $C F(t), t=1, \ldots N$ for current I

4. Compute PV for current I.

5. Store PV in labelled common PL2 for current I.

6. increment I and go to 2 .

Thus, each time the calculation of PV is made, the user is only accessing the data needed for that calculation.

To fill and store data, it is clear that the data transfer program needs to know four things:

1. Which labelled common is being filled or stored.

2. Which set in the data base the labelled common accesses.

3. Which subset of that set is being accessed.

4. Which order the values in the data base must be accessed.

Items 1 and 3 are passed to the routines FILL and STOR as arguments while Items 2 and 4 are derived from information stored in the Labelled Common Size and Labelled Common Order Tables in the Data Base. The next section will explain the construction and function of these tables.

2. LCS and LCO Tables.

Together, the Labelled Common Size (LCS) and Labelled Common Order ( $L C O)$, tables contain enough information to define the labelled commonto-set mapping desired by the user. 
Before we can explain the construction of the LCO and LCS tables in detail, we must develop some terminology. Each dimension has a particular status within each labelled common. If all the data in a given labelled common is independent of dimension $i$, then $i$ is an independent dimension for that labelled common. Otherwise $i$ is a dependent dimension in that labelled cormon. If $i$ is a dependent dimension in labelled common $n$ but $n$ contains data for only one value of $i$, then $i$ is a fixed dimension in labelled common $n$. If $n$ contains variables for more than one value of dimension $i$, then $i$ is a variable dimension in labelled common $n$.

These definitions can be made more formal. Let $x_{n}$ be a piece of data in labelled common $n$. Let $\bar{k}\left(x_{n}\right)=\left(k_{1}\left(x_{n}\right), k_{2}\left(x_{n}\right) \ldots k_{n}\left(x_{n}\right)\right)$ be the set of dimensional values which uniquely indexes element $x_{n}$ in the hierarchical tree. Let $L C_{n}$ be labelled common $n$. Then,

1. $L C_{n}$ is independent of dimension $i$ if: $k_{j}\left(x_{n}\right)=0$ for all $x_{n} \leq L C_{n}$

2. $L C_{n}$ is dependent on dimension $i$ if:

$$
k_{j}\left(x_{n}\right)>0 \text { for all } x_{n} \varepsilon L C_{n}
$$

3. dimension $i$ is fixed in $L C_{n}$ if:

$$
k_{i}\left(x_{n}\right)=k^{*}>0 \text { for all } x_{n} \varepsilon L C_{n}
$$

4. dimension $i$ is variable in $L C_{n}$ if:

a. $k_{i}\left(x_{n}\right)>0$ for all $x_{n} \varepsilon L C_{n}$

b. $k_{i}\left(x_{n}\right) \varepsilon\left(k_{1}^{\star}, k_{2}^{\star} \ldots\right)$ for $x_{n} \varepsilon L C_{n}$ where $k_{i}^{*}, k_{2}^{\star} \ldots$ are integer values.

Note that no labelled common can be independent of attribute since there is no zero branch at the lowest level of the hierarchy. The status of each dimension in each labelled common defined in the previous section is shown in Table IV.B.2. 


\begin{tabular}{|l|c|c|c|}
\hline \multirow{2}{*}{$\begin{array}{l}\text { Labelled } \\
\text { Common }\end{array}$} & Attribute & Dimension \\
\cline { 2 - 4 } GLOB1 & variable & independent & independent \\
PL2 & fixed & independent & fixed \\
PLTI3 & fixed & variable & fixed \\
\hline
\end{tabular}

Table IV.B.2: LABELLED COMMON STRUCTURE

The labelled common size table contains the basic information about how many individual components can occur along each dimension in each labelled common. It also tells which dimensions are dependent and which are independent for each labelled cormon. Let $\operatorname{LCS}(I, J)$ be the $I, J^{\text {th }}$ el ement in the labelled common size matrix where $J$ runs over the labelled commons in the model, $J=1,2, \ldots$ NLC and I runs over the dimensions, $J=1,2, \ldots$ NDIM. If $\operatorname{LCS}(I, J)>0$, then we know that labelled common $\mathrm{J}$ will contain, at most, $\operatorname{LCS}(I, J)$ elements along the $I^{\text {th }}$ dimension. We will call $\operatorname{LCS}(I, J)$ the size of dimension I in labelled common $\mathrm{J}$.

Returning for a moment to the terminology of Chapter II, a labelled cormon simply accesses a subcube of a data set in the data base. The 
number of dimensions in the subcube accessed by labelled common $J$ is the number of variable dimensions in $J$. But, $\operatorname{LCS}(I, J)>0$ if and only if I is variable in $J$. Thus;

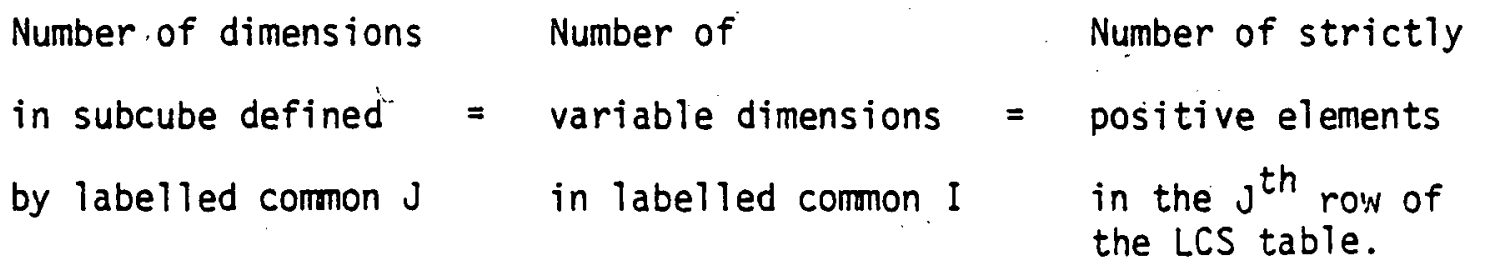

$\operatorname{LCS}(I, J)$ has the natural interpretation of being the length of dimension I on the subcube defined by labelled common J. This size can be defined to be either larger, smaller, or the same as the corresponding length of the same dimension in the data set which is being accessed. If $\operatorname{LCS}(I, J)$ is smaller than the corresponding length in the data set, then the transfer program will move only the values under the first $\operatorname{LCS}(I, J)$ branches at that level of the tree and ignore the rest. For instance, assume a data base has ten time periods and dimension 3 is time. Then, if $\operatorname{LCS}(3,5)=8$, labelled common five will only contain data for the first eight periods. If $\operatorname{LCS}(3,5) \geq 10$, labelled common five would contain values for all ten time periods.

Usually (but. not always) the modeler will want a given labelled cormon to access data for all values of a variable dimension. If that dimension takes on the same number of values everywhere in the model (if, for instance, there are ten time periods at each location in a dynamic regional mode $]$ ), then, setting $\operatorname{LCS}(I, J)$ is easy: simply set it to the number of values dimension I takes on. Setting $\operatorname{LCS}(I, J)$ 
less would ignore some data while setting it higher would result in storage inefficiencies.

The situation is not always so simple, however. In many cases, the number of descendent branches from a node varies across the same level. For example, consider a two-level regional model which has dimension 5 as state and dimension 4 as county. Assume also that labelled common 3 has the variable COWPOP which is a one-dimensional array (vector) of populations by county for a given state. What should $\operatorname{LCS}(4,3)$ be? clearly the number of counties is not constant across states, in fact, it varies widely (from 3 in Delaware to 254 in Texas). To include data for all the counties in each state, $\operatorname{LCS}(4,3)$ must be equal to or greater than the maximum number of counties in any state (i.e., 254). Thus to access all the data along a given dimension $\operatorname{LCS}(I, J)$ must equal the maximum number of values dimension I can take on.

The Labelled Common Size Table for the example in Figure IV.B.1 is shown in table IV.B.3.

\begin{tabular}{|lc|c|c|c|}
\hline \multicolumn{2}{|c|}{$\begin{array}{l}\text { Labelled } \\
\text { Common J }\end{array}$} & \multicolumn{3}{|c|}{ Dimension I } \\
\cline { 2 - 5 } & Attribute 1 & Time 2 & Plant \\
\hline GLOB1 & 1 & 2 & 0 & 0 \\
PL2 & 2 & 1 & 0 & 0 \\
PLTI3 & 3 & 1 & 50 & 0 \\
\hline
\end{tabular}

Table IV.B.3: SAMPLE LCS TABLE 
The remaining information needed to set up the labelled commonto-set mapping desired by the user is stored in the labelled common order table ( $L C O)$. The labelled common order table defines the order in which elements are mapped from the data base into each labelled common. The I, Jth element of the labelled common order table is denoted $L C O(I, J)$ and refers to the order of the $I^{\text {th }}$ dimension in the $J^{\text {th }}$ labelled common. For example, consider a model which has two attributes varying by five time periods. The labelled common which contains this data could be set up in two different ways.

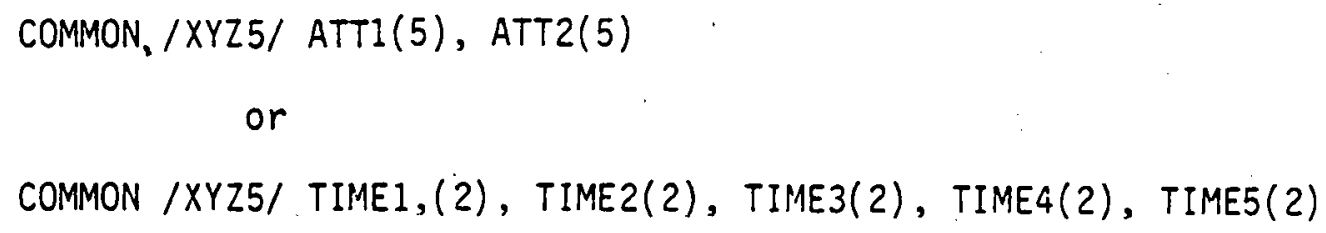

In the first case, the data is placed into two vectors, each representing values of one attribute for all five time periods. In the second case, each vector contains the values of both attributes for a given time period. In the first case, the order of the time dimension would be 1 and the order of the attribute dimension 2 in common XYZ5. In the second case the order of the time dimension would be 2 and the order of the attribute dimension would be 1 .

At the lowest level, not only does each labelled common define a subcube of data, but each array and variable within a labelled common addresses a subcube whose dimensionality is the same as the dimensionality of that array. For indexing purposes, the dimensions in each array must be ordered. For example, a matrix of the populations of five regions 
for three time points can either be set up as $\operatorname{POP}(5,3)$ or as $\operatorname{POP}(3,5)$. In FORTRAN arrays, dimensions are ordered from left to right. Thus, in the first case, the order of the region dimension would be 1 and the order of the time dimension 2. Now, consider the labelled common that contains two attributes: GNP and Population (POP) for five regions for three time periods. This data could be organized in several ways. Some of these, with the corresponding LCO table rows, are shown in Table IV.B.4.

\begin{tabular}{|c|c|c|c|}
\hline & Attribute & Time $\quad 2$ & Region 3 \\
\hline $\operatorname{DATA}(5,3,2)$ & 3 & 2 & 1 \\
\hline $\operatorname{DATA}(3,5,2)$ & 3 & 1 & 2 \\
\hline $\operatorname{POP}(5,3), \operatorname{GNP}(5,3)$ & 3 & 2 & 1 \\
\hline $\operatorname{POP}(3,5), \operatorname{GNP}(3,5)$ & 3 & - & 2 \\
\hline
\end{tabular}

Table IV.B.4 LCO TABLE OPTIONS

One more piece of information is contained in the LCO table: which dimensions are fixed in each labelled common. If dimension I is fixed in labelled common $J$, then $L C O(I, J)$ should be set by the user to be equal to the arithmetic inverse of the actual order. To illuistrate this requirement, we have set up the LCO table for the two-plant cash. flow example in Table IV.B.5. Notice that the value in the plant dimension is negative for labelled commons. two and three since that dimension is fixed in those labelled.commons. 


\begin{tabular}{l|c|c|c|}
\cline { 2 - 4 } $\begin{array}{l}\text { Labelled } \\
\text { Common }\end{array}$ & \multicolumn{3}{|c|}{ Dimension } \\
\cline { 2 - 4 } GLOB1 & Attribute & Time & Plant \\
\cline { 2 - 4 } PL2 & 1 & 0 & 0 \\
PLTI3 & 2 & 0 & -2 \\
& 1 & 1 & -3 \\
\hline
\end{tabular}

Table IV.B.5 LABELLED COMMON ORDER TABLE

The rules for constructing the LCS and LCO tables are shown in Table IV.B.6.

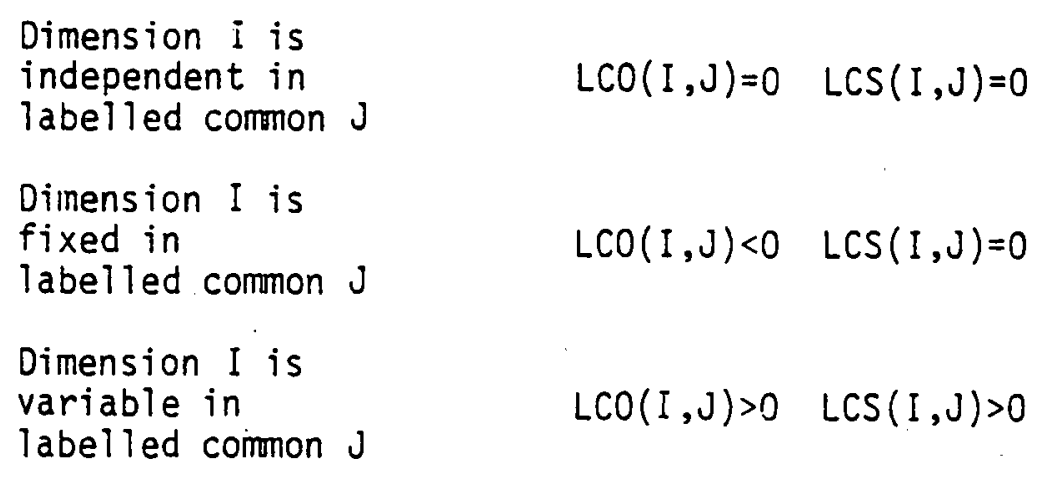

Table IV.B.6 LCO AND LCS CONVENTIONS

A fourth case in which $L C O(I, J)$ is less than 0 occurs when selective filling or selective storing is used. This case will be discussed in Chapter VI. Selective filling and storing have been designed to Jecrease the number of computations for FILL in certain situations but are not required to run a model. 


\section{Main Program}

The main program of a model sits at the highest level of the program hierarchy and passes control from data transfer routine SOFTIN to the FORTRAN logic of the model and then to data transfer routine SOFTOU. A sample main program for the cash flow example is shown in Figure IV.B.2. The main routine consists of four parts:

a. Labelled Common Block

The labelled common block contains all the labelled commons of the model in order. The first labelled common; COMMON/FIRST/SS(2), is a durmy, followed by the rest in the mumerical order. They appear as rows in the LCS and LCO tables. The size specified for each one here must equal the size implied by the LCS table; i.e., the product of the absolute values of the nonzero entries in the corresponding row in the LCS table.

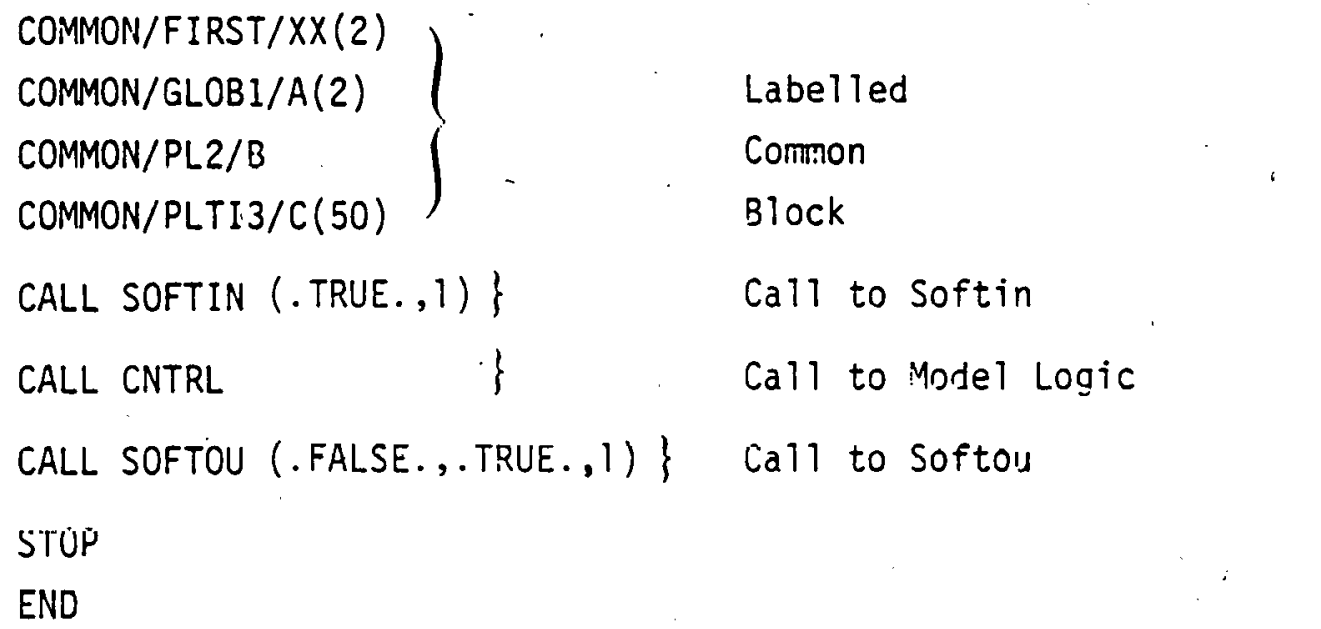

Figure IV.B.2 MAIN PROGRAM 
b. Call to SOFTIN

This statement calls subroutine SOFTIN which reads in model

data. The statement has the following syntax:

CALL SOFTIN(STRIN,MODELIN)

STRIN is a logical variable with the following usage:

STRIN=. TRUE. Data is read in from file FTO02

which is the result of a System 2000 unload.

STRIN=.FALSE. Data is read from the binary file

FT003.

MODELN is the integer model number of the current model. If MOOELN is set negative, the transfer program will prompt the user for the settings of its internal write flags.

c. Call to Model Logic

d. Call to SOFTOU

This statement calls subroutine SOFTOU which writes out model data. The statement has the following syntax:

CALL SOFTOU (RDOUT, STROUT,MODELN)

RDOUT and STROUT are both logical variables with the following. usages:

RDOUT $=$. TRUE. saves data in the binary file FTO03

STROUT=.TRUE. saves data in the file FTOO4 in a

format that can be loaded into a System 2000 data base. If both STROUT and RDOUT are set to .FALSE., the results of the model are not saved. MODELN is the model number as above. 


\section{Calling FILL and STOR}

The final step remaining to the programmer is to insert the calls to the routines FILL and STOR into his FORTRAN program. As mentioned above, FILL is called whenever the program requires the data in a new labelled common. STOR is called whenever data is to be replaced in the data base.

Subroutine FILL has the following arguments:

\section{CALL FILL(LCNUM, IDV)}

where LCNUM is the number of the labelled common to be filled and IDV is a vector that gives the values of the fixed dimensions which are to be filled over. The FILL routine determines which variables are to be fixed or free accord:ng to the "truth table" in Figure IV.B.3.

Consider the present value example developed in this section. At some point in his program, the modeler will wish to access the cash flow vector CF for a given plant. To do this, he will need to call FILL on labelled common PLTI3 which has dimension 3 (Plant) as fixed. Exactly which value of dimension 3 he desires to obtain data for is communicated to subroutine FILL by means of a vector, called the IDV vector, below:

$$
\operatorname{IDV}(3)=2
$$

$$
\text { CALL FILL }(3, I D V)
$$

This call will bring the cash flow stream for plant number 2 in to labelled common PLTI3. It does so by fixing the value of dimension 3 at 2 . 
Dimension $J$ in Set I

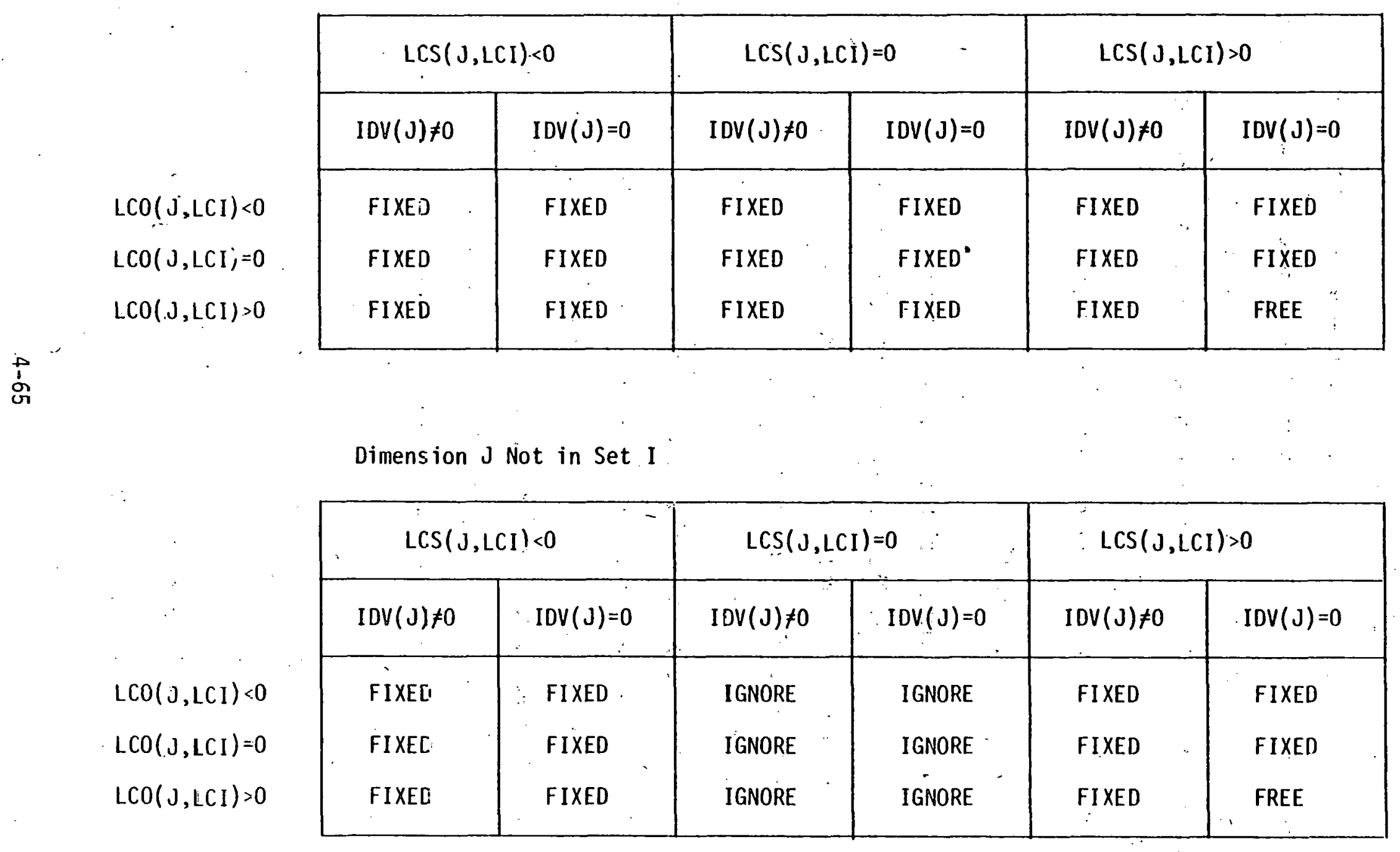

Figure IV.B.3: FILL TRUTH TABLE 
Subroutine STOR is used to place model results from the model into the data base. The arguments for STOR are the same as those for FILL:

\section{CALL STOR(LCNUM, IDV)}

A call to STOR replaces the values in labelled common LCNUM into a position in the data base specified by the vector IDV.

The principles of filling and storing labelled commons are illustrated in Figure IV.B.4. This program was written to compute the net present values of cash flows for two plants according to the example developed in this section.

Notice that IDV is 'empty' (has no assigned values) when labelled common GLOBI is filled. This is because GLOBI has no fixed dimensions. In a given call to FILL, those dimensions for which IDV is non-zero will be assumed to be fixed in that labelled common. It must contain a zero value in each position corresponding to a free dimension. Since, in the example, both PL2 and PLTI3 have dimension 3 fixed, IDV( 3$)$ is assigned a positive value for the corresponding calls to FILL and STOR. 


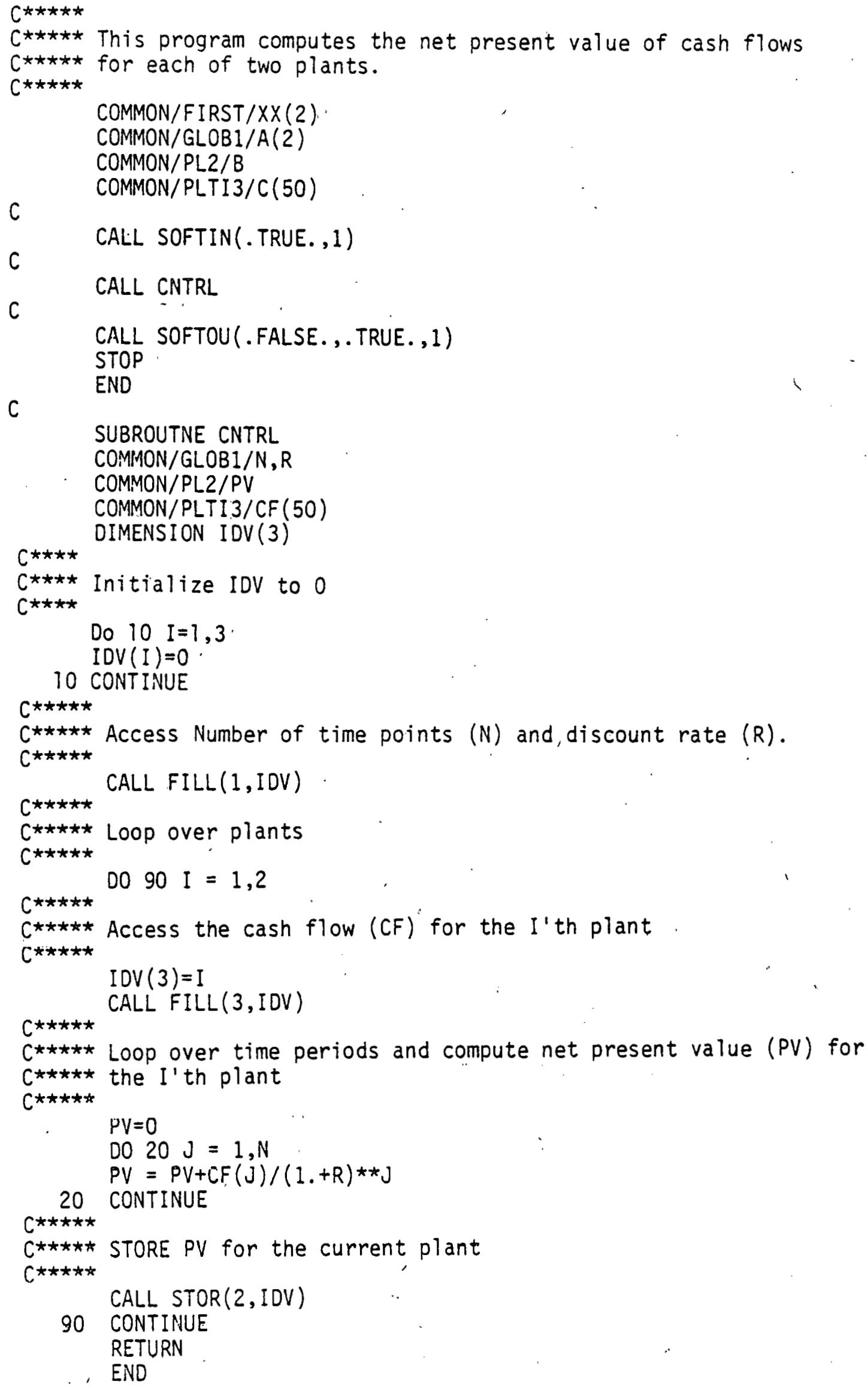




\section{CHAPTER $V$}

\section{EXECUTIVE PROGRAMMER'S GUIDE}

The following information is presented to facilitate the understanding of the main algorithms of the EXECUTIVE code. Because the code utilizes the System 2000 Procedural Language Interface (PLI), the reader is advised to familiarize himself with both System 2000 and the PLI. Sections 1.0 - 1.3, $2.0-2.3,3.0,3.3$ and 3.4 of System 2000 Extended Procedural Lanquage Interface Feature (MRI publication UM - 1) should be carefully read and understood. The subsections on linked storage areas, automatic return code processing, locate files and the RELEASE, SAVE, RESTORE, LOCATE, ORDER, and GET commands may be skipped as none of these features has been utilized in the Executive System. We further suggest that the reader become familiar with Chapter IV above concerning use of the system, as a good intuitive understanding of the aims of the system facilitates the more difficult discussions that appear in this chapter.

\section{A. PROGRAMMING CONVENTIONS}

Embodied in the Executive computer code are a number of programming conventions which, if understood and adhered to, can lead to an orderly understanding and evolution of the code as new capabilities are added. First, the order of FORTRAN statement labe'ls is important. If statement label $i$ is less than statement label $j$, then the statement corresponding to label $i$ must by convention appear before the statement corresponding to label $j$. Rightmost zeroes are ignored in determining the order so that 10 (equivalent to 1) comes, before 2, and 9100 (equivalent to 91 ) precedes 92. 
In debugging code it is often necessary to insert WRITE statements to check intermediate results. Rather than deleting these WRITE instructions when the output is no longer desired, an array of logical flags has been incorporated. These flags allow the user to select which, if any, IRITE statements will operate during execution of the program. This has the advantage that, if a particular WRITE statement is regarded as no longer necessary, then the corresponding logical flag need only be turned off, thereby avoiding the necessity of changing code and recompiling. Also, the intermediate results are easily accessible to anyone learning or developing the code. The array that holds these flags is named WRT and is passed to subroutines via the WRTBK labeled common. The label of the corresponding FORMAT statement is determined by adding 900 to the index of WRT which controls the output. For example, if the values of array MATRIX are to be output for inspection, the code would be similar to the following:

$$
\operatorname{IF}(\operatorname{WRT}(11)) \text { WRITE }(6,911) \text { NiATRIX 、 }
$$

911. FORMAT ( ', $8(18,2 X))$

Table V.A.1 lists the active indices of the write flags in WRT and describes the output each controls.

In addition to flagged WRITE statements controlling intermediate results, the program also contains several unflagged WRITE statements. For example, the statement in the main routine which outputs the 'EXEC>' prompt falls into this category. The labels of the FORMAT statements which correspond to these unconditional WRITE's are restricted to the 800 range. 
In designing and implementing this interactive program, errors on the user's part must be detected during the parsing of imput lines. Each error requires the generation of a diagnostic to inform the user of the nature of his error. All such massages are generated in the code by issuing a call to the routine PMSAG (print a message). This subroutine uses the index IMSAGE, passed as a parameter, to determine which message to print. In the code, when an error has been encountered, a GO TO is executed. The destination of this GO TO is the appropriate call to PMSAGE. The state labels which are the objects of these GO TO's are restricted to the 700 range.

This, together with the restriction that the labels of all statements not falling into any of the above three classes be in the 100 to 600 range, completes the list of conventions.

\begin{tabular}{|c|c|c|}
\hline Value & Action & Discussed in Section \\
\hline 1 & Primary area pointers & V.B \\
\hline 2 & Set parameter values & IV.A.3.C.i \\
\hline 3 & Primary area offsets & V.B \\
\hline 4 & $\begin{array}{r}\text { How much of Workspace } \\
\text { Printed }\end{array}$ & IV:A.3.C.ii \\
\hline 5 & $\begin{array}{r}\text { I/0 tables of GTAB \& } \\
\text { PTAB }\end{array}$ & IV.A.2.a \\
\hline
\end{tabular}




\section{B. THE PRIMARY AREA}

The primary area, or workspace as it is called in the EXECUTIVE User's Guide, is a data structure second in importance only to the data base. It consists of the ten components shown in Figure V.B.1. The sizes of most of the components are variable (see the SIZE routine) making it necessary to design a means for allowing this data structure to change. All of the components are stored in one FORTRAN vector. This vector is named IPAREA and resides in the PRAREA labelled common. Another labelled common, PAPTRS, contains a set of pointers, one for each component. The dimensions of the components are stored in two label led commons, GCONST and PADIMS. The former contains those dimensions which remain constant throughout the running of the EXECUTIVE and the latter contains those which change via the SIZE command. Below is a brief description of each component along with its pointer and dimensions.

1) Elements

\section{Pointer: IPTBPT}

Dimensions: NRWSPA, NCLSPA

The elements matrix holds all data values which are to reside at the lowest level of the hierarchical tree. Each element may be an integer, floating point number, or special default string. This default string is recognized by the EXECUTIVE code when it is placing values in the data base and it signifies to the program that no value is to be placed in the data base for that element. 


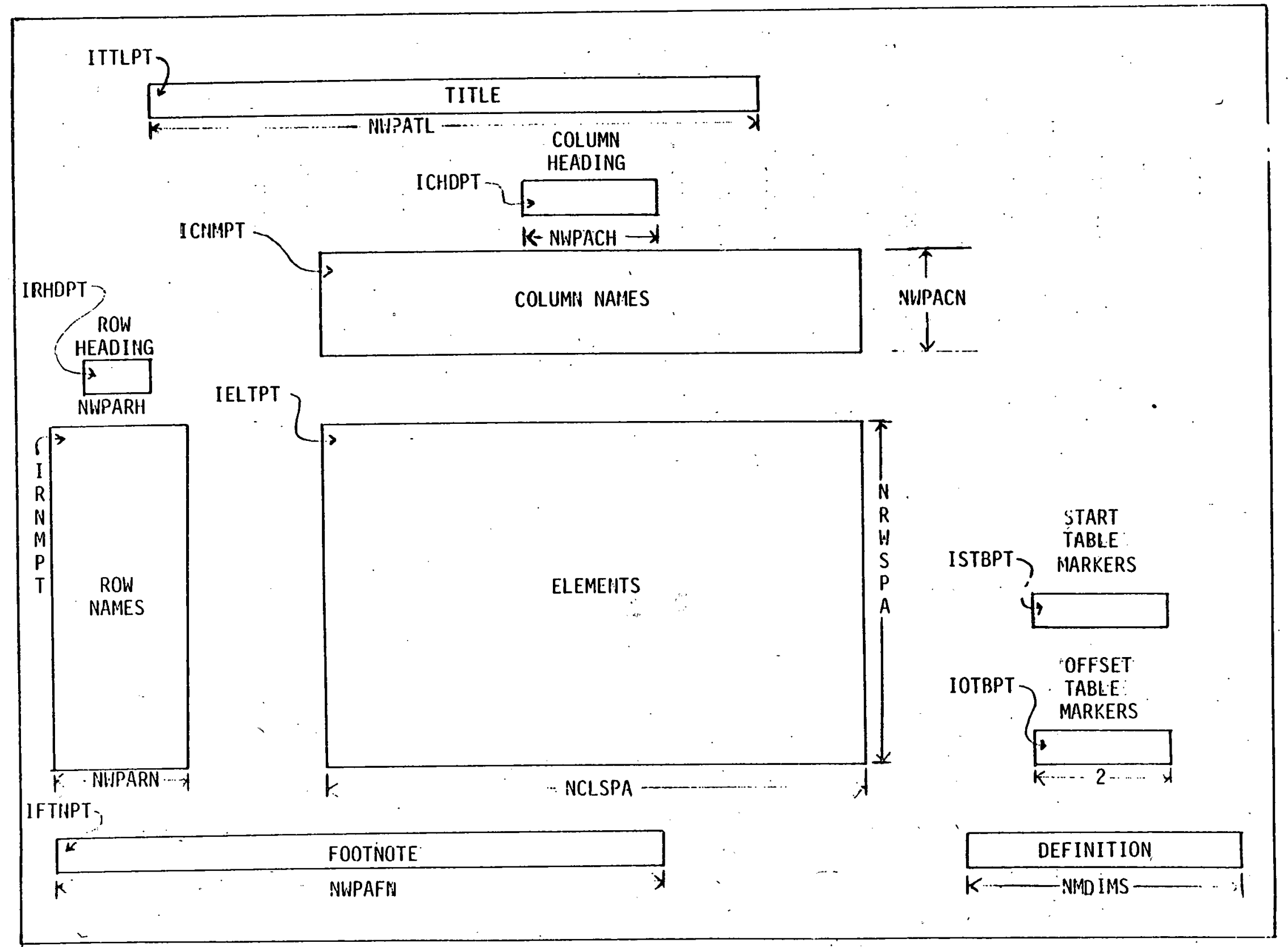

Figure V.B.I. THE PRIMARY AREA 
2) Row Names

Pointer: IRNMPT

Dimensions: NWPARN, NRWSPA (for hierarchical tables)

NWSTNM, NRWSPA (for system tables)

The row names reside in a vector of text strings. These text strings are the names which the user has chosen to give the rows of the elements matrix. 'Each element of this vector has NWPARN words. All of this space is used for hierarchical table row names but only NWSTNM words are used for system table names. NWSTNM is restricted to be less than or equal to NWPARN.

3) Column Names

Pointer: ICNMPT

Dimensions: NWPACN, NCLSPA (for hierarchical tabies) NWMDNM, NCLSPA (for system tables)

These names also reside in a vector of text strings and are the names which the user has chosen to give the columns of the elements matrix. As with row names above, NWDMNM is restricted to be less than or equal to NWPACN.

4) Row Heading

Pointer: IRHDPT

Dimensions: NWPARH

The row heading is a text string containing the name of the hierarchical dimension which varies across the rows of the elements matrix.

5) Column Heading

Pointer: ICHOPT

Dimension: INWPACH 
The column heading is also a text string containing the name of the hierarchical dimension which varies across the columns of the elements matrix. See section V.0.2.a. for a discussion of the concept of rows and columns varying across hierarchical

1 dimensions.

6) Title

Pointer: ITTLPT

Dimensions: NWPATL

The title is a text string containing the title of the table currently residing in the primary area.

7) Footnote

Pointer: IFTNPT

Dimension: NWPAFiN

The footnote is a text string containing footnote to the table currently in the primary area.

8) Definition

Pointer: IDEFPT

Dimensions: NMDIMS (resides in MDINFO labelied common). This vector contains the $1 / 0$ table vector which defines the data currently in the primary area. See Section V.D.1 for a discussion of how these $1 / 0$ vectors are used.

9) Start Table Markers

Pointer: ISTBPT

Dimension: 2

If a user retrieves a table and specifies a starting row or column other than 1 , this information is stored in the start table markers component. 


\section{0) Offset Table Markers}

Pointer: IOTBPT

Dimension: 2

If there exists a row with index $i$ such that row $i$ contains a non-default element but rows $j, i+1 \leq j \leq N R W S P A$, are all defaults then the index $i$ is saved in the offset table markers. The same is true of columns. These markers give what is referred to as the true table bounds as illustrated in the figure below.

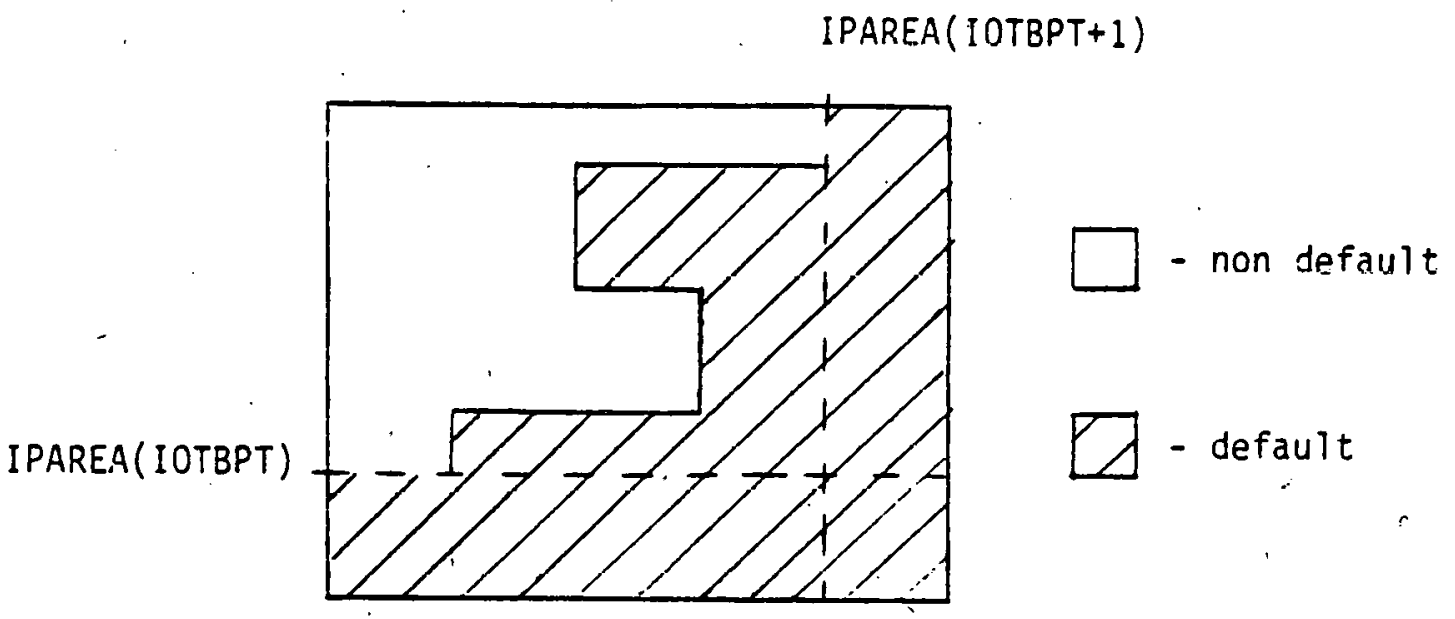

Figure V.B.2. THE TRUE BOUNDS OF A TABLE

The SIZE subroutine shows how the primary area dimensions are used to set the pointers. We will close this section with an example of how components are passed to subroutines.

Example

Subroutine $x$ requires the elements matrix, row names, and column names. The calling routine will be as follows:

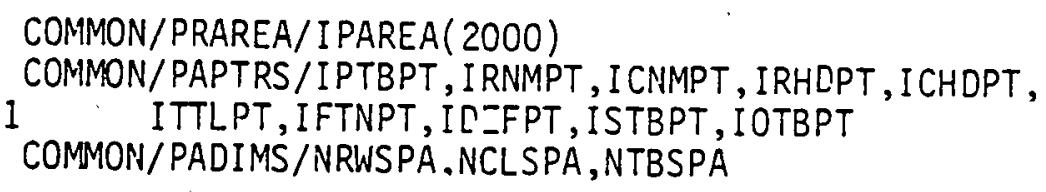


COMMON/GCONST / NWDSPA, NWPARN, NWPACN, NWSTNM,

1 NWMDNM, IWPARH, NWPACH, NWPATL, NWPAFN, NWMNAM

CALL X(IPAREA(IPTBPT), I PAREA(IRNMPT),

1 IPAREA (ICNMPT), NRWSPA, NCLSPA, NWPARN,

2 NWPACN)

The object subroutine would be as follows:

SUBROUTINE X(ELMNTS, RWNAMS, CLNAMS,

1 NRWSPA, NCLSPA, NWPARN, NWPACN)

INTEGER ELMNTS (NRWSPA, NCLSPA), RWNAMS (NWPARN, NRWSPA),

1 CLNAMS (NWPACN,NCLSPA) 
C. CONTROL AND PARSING

This section deals with the high level algorithms of the EXECUTIVE code. The discussion focuses on how the flow of control from the high level to low level routines is accomplished. Subsection V.C.I describes how the subroutine PARSE is used to determine what the EXECUTIVE user has input. Subsection V.C.2 then shows how the results of PARSE pass control to the appropriate routines.

\section{Subroutine PARSE}

The function of subroutine PARSE is to read a line input by the user and then call PARSE once for each of the successive parameters in the 1 ine. Parameters, which are parsed from left to right, are defined to be punctuation marks only or integers, reals, names or text strings followed by punctuation marks. Consider the following line:

$$
C, R, 1 / 0,0,4.58,0
$$

The parameters are parsed as follows:

Call to PARSE

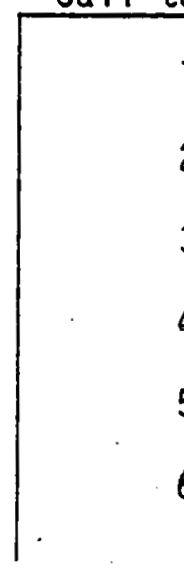

PARAMETER

c,

R,

I/

o.,

4.58,

$0 E O L^{\star}$

TYPE

Name/punctuation

Name/punctuation

Integer/punctuation

Red l/punctuation

Real/punctuation

Integer/punctuation

*End of Line 
In the EXECUTIVE code, whenever user input is required,it is read into the array INPTLN. (input line) which resides in labeled common LINBK. This array contains 1.8 words so that on an IBM 360 or 370 the maximum number of characters that can be input at any one time is 72 . In order to increase this count to say 80 or 132 characters, it would be necessary to do more than just increase the dimension of INPLIN and the indices of the implied DO-loops which control the reading into INPTLN. IBM input buffers for unit 5 on TSO default to 72 characters. Attempts to read any more than 72 characters into one line will. result in an IBM input/ output error message.

The information about the parameters of INPTLN is returned in the members of the PARSEP. labeled common. This labeled common is declared with the same variable names in all routines. The declaration is as follows:

COMMON/PARSEP/ IWRDPT, ICHRPT, INPUNC, ITYPAR, IWDERR, LINEND, ITEXTF, 1 IPRSTR(20), INTNUM, REALIIM

A11 of PARSEP's members are of type integer with the exception of REALNM (real number) which is type real. 'A brief explanation of each member of PARSEP fOllows.

1) IWRDPT (word pointer), ICHRPT (character pointer)

These two components together determine a unique character of INPTLN. IWRDPT points to a word of INPTLN and ICHRPT points to a byte, or character, of that word (i.e., of INPTLN(INRDPT)). The character pointed to is the 
first character to be examined on the next call to PARSE. After a new input line has been read, these two variables must be initialized to 1 .

2) INPUNC (index to punctuation)

This member provides an index to the type of punctuation mark parsed with the last parameter. The following table lists the possible values of INPUNC and the corresponding punctuation.

\section{INPUNC}

\begin{tabular}{|c|c|}
\hline Values & Punctuation Mark \\
\hline 1 & comma \\
2 & period \\
3 & minus sign/hyphen \\
4 & equal sign \\
5 & plus \\
6 & asterisk \\
7 & slash \\
8 & left parenthes is \\
9 & right parenthesis \\
10 & semi-colon \\
11 & colon \\
12 & hash mark
\end{tabular}


3) ITYPAR (index to type of parameter)

Indicates the type of parameter parsed from INPTLN. The following table lists the possible values of ITYPAR and the corresponding parameter type.

ITYPAR

\begin{tabular}{|c|l|} 
Value & Parameter Type \\
\hline 1 & name and punctuation \\
2 & punctuation only \\
3 & floating point and punctuation \\
4 & integer and punctuation \\
text string and punctuation
\end{tabular}

4) IWDERR - not used

5) LINEND (1 ine end marker)

A value of 1 indicates that the end of the line has been read. A value of 0 implies the opposite.

6) ITEXTF (text flag)

The difference between names and text strings is that blank characters are suppressed from names but kept in text strings. When a call to PARSE is expected to return a text string, ITEXTF must be set to 1 , and when any other parameter is expected, ITEXTF must be set to 0 . In (1) above, ITEXTF was 0 before all 6 calls to PARSE. 
7) $\operatorname{IPRSTR}(20)$ (the parsed string)

This array returns the name or text string parsed.

8) REALNM (real number), INTNUM (integer number)

If ITYPAR $=3$, then REALNM returns the corresponding floating point number; if ITYPAR $=4$, INTNUM returns the corresponding integer.

\section{An Example of Flow of Control}

The preceding section described the information available via the PARSE routine. We now discuss, through an example, how this information is used. Figure V.C.1. Shows the syntax of the CHANGE command. Because it is a flow chart, this diagram serves as a better illustration of the flow of control than its counterpart in Section IV.A.3.b.iii.

After an input line has been read, and both IWRDPT and ICHRPT set to 1, PARSE is called. The first component of every command line is of type name so this initial call would be as follows.

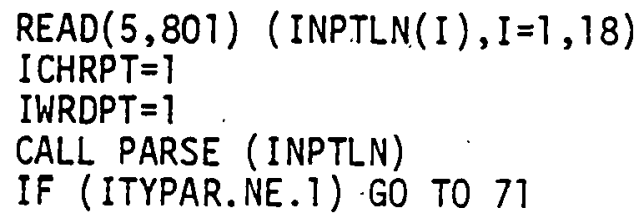

Note that the last 11 ne checks for an error condition, namely that an invalid paramenter type has been encountered, and if that condition exists a GO TO is executed. This is in accordance with the conventions discussed in Section V.B. 


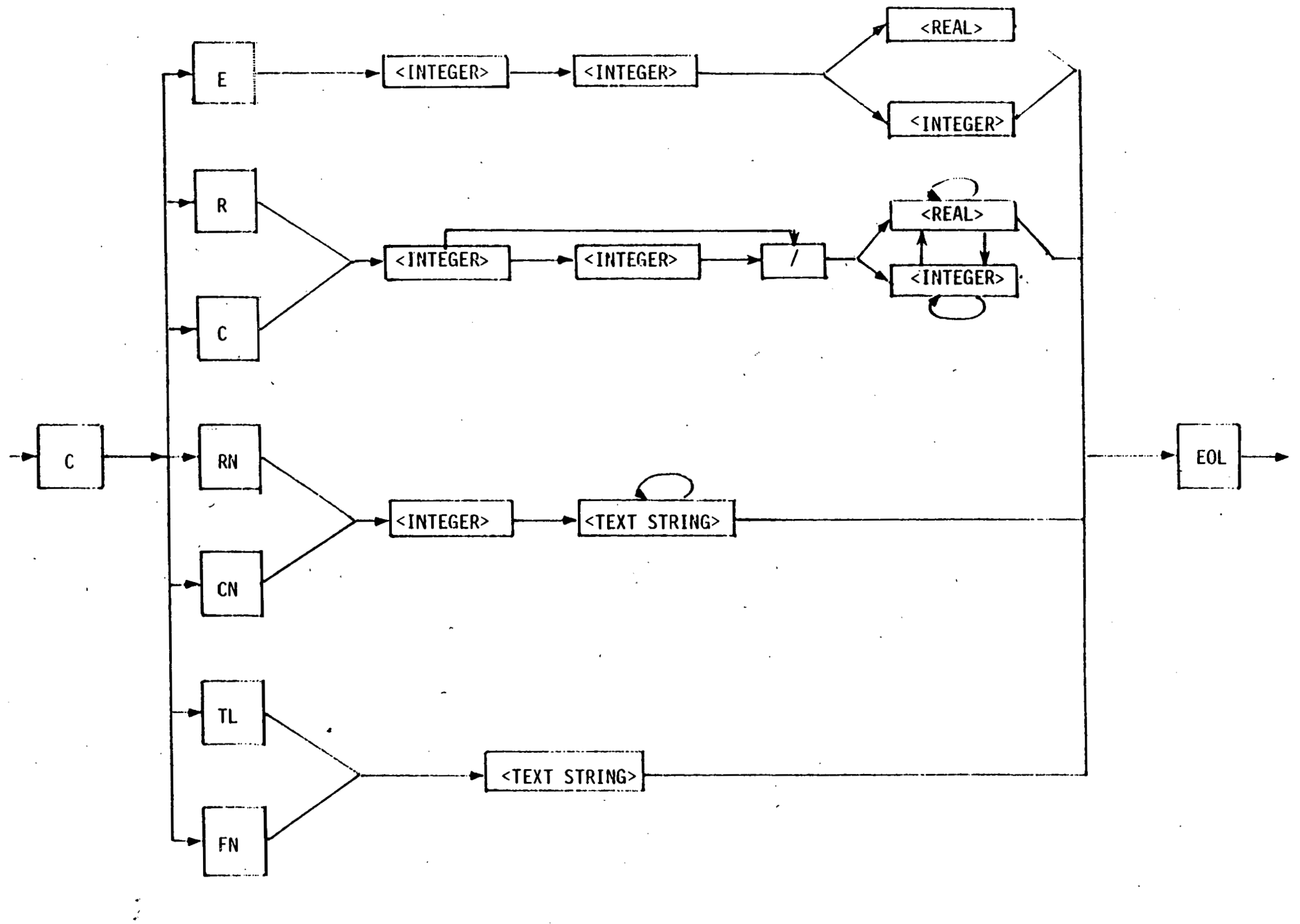

Figure V.C. I. SYNTAX OF THE CHANGE COMMAND 
Now, assuming a CHANGE command was issued, the string ' $C$ ' with trailing blanks is residing in IPRSTP. Once this has been determined by function ICMPST, control is passed to subroutine CHANGE: The first lines of this routine are as follows:

\section{IF(LINEND.EQ.1) GO TO 71 \\ CALL PARSE (INPTLN) \\ IF(ITYPAR.NE.1) GO TO 72}

Again, the parameter must be of type name.

Now assume that after this code executes the string ' $E$ ' is in IPRSTR.

Control is passed one level deeper where the indices of the element to change must be parsed.

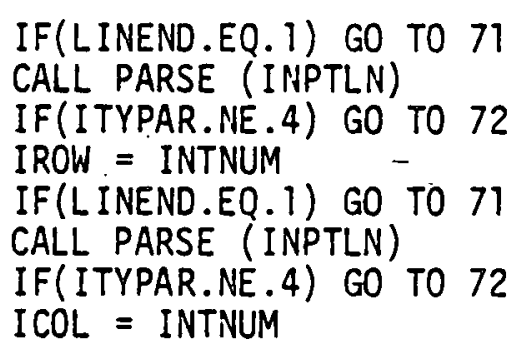

All that remains is to determine the value to assign to the element, which is done as follows.

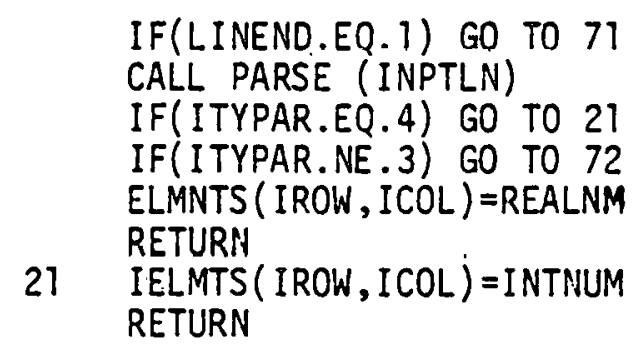

The arrays ELMNTS and IELMTS have been previousiy equivalenced so that the two assignment statements above modify the same word in memory. 
This is a standard technique used throughout the EXECUTIVE code so that integers and reals may reside in the same table.

With the execution of either of the above RETURN's, control returns to the MAIN routine and the whole process is repeated. The reader is urged to inspect the code to see how control is passed for this and other commands. 


\section{DATA BASE INTERFACE}

The CMS data base is best thought of by the programmer as consisting of five trees sharing a common root. (See the data base definition in the appendix and in Chapter VI). The root contains the model repeating group, four of the trees correspond to system tables, and the remaining tree is called the hicrarchical data tree.

Manipulation of the system table trees is quite straightforward. System tables are restricted to two dimensions and hence their tree representations are restricted to two levels. A double loop, such as the one shown below for $1 / 0$ tables, is enough to traverse an entire system table.

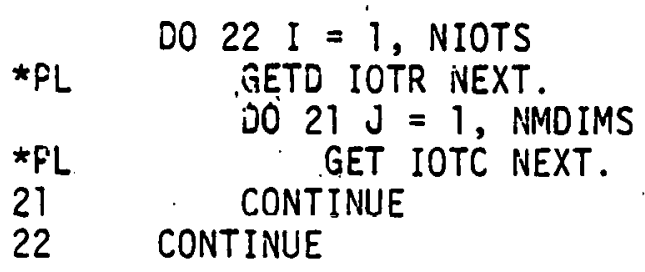

(Note that the statements beginning with '*PL' are System 2000 procedural language statements.)

If a subset of the rows of the table is desired, then it is only necessary to al ter the bounds of index I.

The discussion of the hierarchy, however, is much more complex. Before discussing the hierarchical tree in detail, a few introductory words are in order. When we think of levels in trees, we usually assign the root to level 0 , the roots of $i$ ts immediate subtrees to level 1 , and so forth. In dealing with the hierarchy, it is convenient to use the concept of dimensions instead of levels where dimension 1 corresponds to the lowest level of the 
tree and dimension NMDIMS +1 corresponds to the root. Actually according to the data base definition, every model has 29 dimensions. The logic has been set up to ignore dimensions NMDIMS +1 through 29 however. In order to accomplish this we need only extend one path down to the NMDIMS + ist level when creating the model and then, prior to any traversal of the hierarchy, place a PLI secondary stack pointer to the single node at the NMDIMS + ist leve1. The creation of the path can be found in the CRNWMD subroutine and the placement of the pointers is at the beginning of the GDATA and RDATA routines.

All traversals of the tree are in preorder. This ordering is defined as follows:

1) Visit the root.

2) Traverse, the nodes of the leftmost subtree in preorder.

3) Traverse the nodes of the second leftmost subtree in preorder and continue in this manner until all subtrees have been traversed.

In the forthcoming sections, we will present a number of tree diagrams. In all cases, we will number the nodes in the trees in preorder.

1. Traversing the Hierarchy

The way in which we set up our data structures often differs radically from the way in which the user pictures them. This is definitely the case with the hierarchy. Figure V.D.1. shows the way in which the user might view the data base. It is an ordered tree with the property that a node at any dimension other than dimension 1, may 
have an arbitrary number of immediate subtrees.

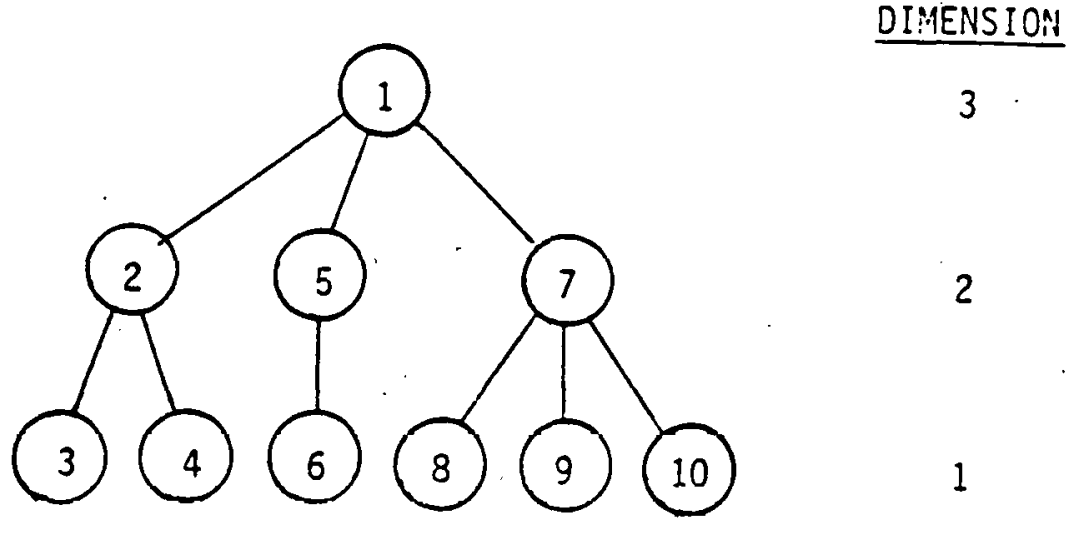

Figure V.D.I. CRDERED TREE

It is sometimes helpful to view the hierarchical tree not as a tree per se but, rather, as a binary tree. It is very convenient to use binary trees because the maximum number of links emanating from any node is always two as we shall see shortly. Constructing a transformation from a given tree to a unique binary tree is straightforward. The links 'First Descendent' and 'Next Sibling' are used. The first descendent link poin'ts to the oldest child of the current node and the next sibling link points to its next oldest brother. It is clear that these links will give the desired transformation. Figure V.D.2 shows the tree in Figure V.D.1 after this transformation has been made. 


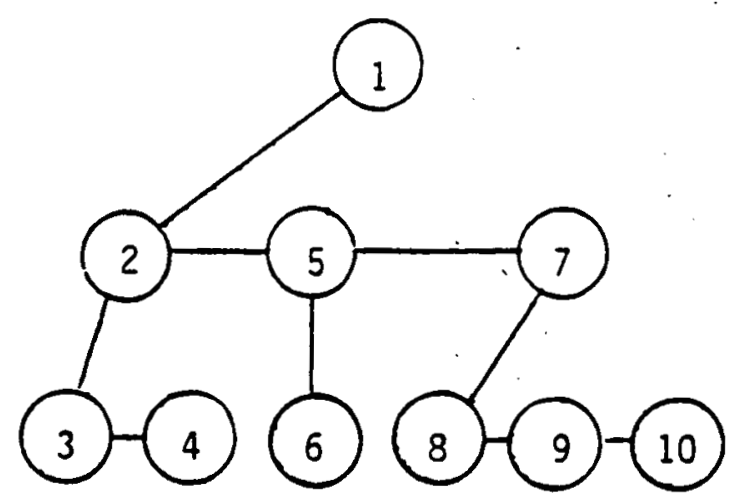

DIMENSION

3

2

1

Figure V.D.2. BINARY TREE

Note that the number of links has not changed al though the average path length from the root to any leaf has increased. The digital representation has become more straightforward, as the only numbers required at each node to define the tree structure are:

$\operatorname{FSTDEC}(X)$

$\operatorname{NXTSIB}(X)$

These two numbers contain sufficient information to traverse the tree. In (1), FSTDEC is a function which returns the location of the first descendent of the node whose location is $X$ and, in (2) NXTSIB returns the location of the next oldest sibling.

The System 2000 PLI GETD S2KCNT option provides a combination of these two operations and is all that is necessary to traverse all or part of the hierarchy in preorder. The only required parameters are dimension number, to indicate which dimension to move across, and the number of moves to make. The following, algorithm describes GETD S2KCNT in detail. 


\section{Algorithm Get Descent}

This algorithm uses the array PATH where PATH(I) contains either the location of a node at dimension I or a $\Lambda$,(nuil) and DIM, the index of the dimension to move across. It is assumed that PATH(DIM = 1) $\neq \Lambda$. If PATH(DIM) $=\Lambda$, then the location, of the Nth son of the node pointed to by PATH(DIM +1$)$ is returned to PATH(DIM), where $N$ is the number of nodes to move across. Otherwise, the location of the next Nth youngest sibling of the node pointed to by PATH(DIM) is returned to PATH(DIM). If the move cannot be made, IRTNCD is set to 4 , otherwise it returns 0 .

1. (Initialize) IRTNCD +0 .

2. (Pointer at $D I M+1$ ?) IF PATH $(D I M+1)=\Lambda$, then error.

3. (Pointer at DIM?) $X+$ PATH(DIM). If $X \neq \Lambda$, then go to 5 .

4. (Get the first son) $X+$ FSTDEC (PATH $(D I M+l)$ ). If $X=\Lambda$, then IRTNCD 4 and return. Otherwise, $N+N-1$.

5. (Done?) If $N=0$, then PATH(DIM) $+X$ and return.

6. (move to right) $X+N X \operatorname{NSIB}(X)$. If $X=\Lambda$, then, IRTNCD +4 and return. Otherwise, $N+N-1$ and go to 5 .

A slightly stronger version of the above algorithm appears in the PLI routine GTDCNT. The PLI stack replaces the array PATH. What makes the routine stronger is that the assumption that $\operatorname{PATH}(D I M+1) \neq \Lambda$ may be discarded. However, this is of no great advantage, as the only two calls to GTDCNT which violate this assumption are those at the beginning of 
the RDATA and GDATA routines which place a secondary stack pointer at the single node at dimension NMDIMS +1 as mentioned in Section V.D.

Let us turn now to the Input/Output table and see how it is used to traverse the hierarchy. Each row of the I/O table is a vector whose NMDIMS elements are integers. If all of the elements are positive, then that row of the I/O table defines a path from the root to a leaf of the hierarchy and, hence, defines a data point. An example of the correspondence between such a row of the $1 / 0$ table and a tree path is shown in Figure V.D.3. Each element of the I/O table now corresponds to a dimension of the hierarchy.
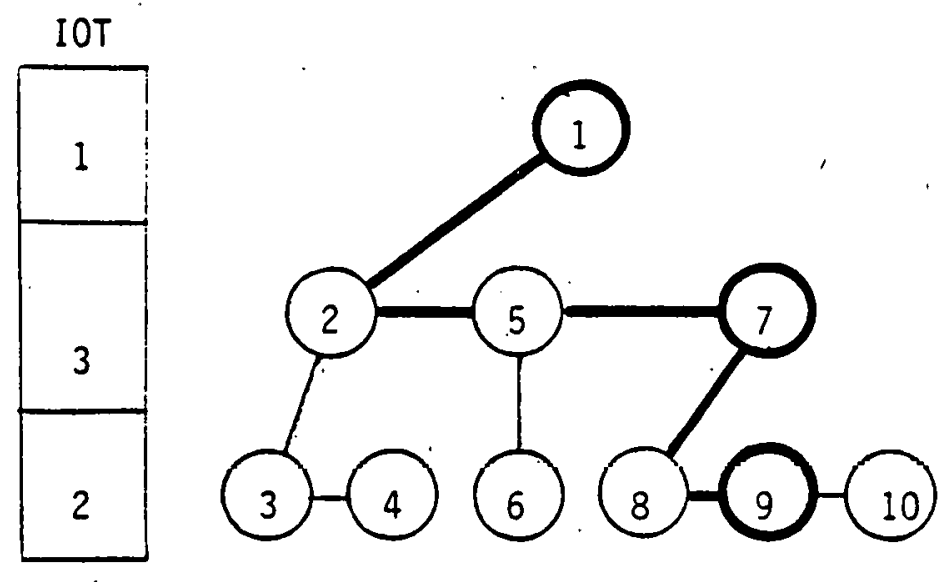

DIMENSION

Figure V.0.3. CORRESPONDENCE BETNEEN IOT: AND TREE PATH 
To determine the path defined by a row of the $1 / 0$ table with positive entries, it is best to start at the root and work downward. IOT(NMDIMS) tells how many children of the root to move across. IOT(I) tells how many. children of a previously determined node at dimension $I+1$ to move across. Algorithm Traverse Path below makes this more precise.

\section{Al gorithm Traverse Path}

Given the vector IOT with NMDIMS positive elements, the path defined by this $I / O$ table vector is traversed. ROOT is the location of the root. Algorithm Get Descendent is called as a subroutine (GETD). After Algorithm Traverse Path has run, the array PATH will contain the desired path.

1. (Initialize.) DIM + NMDIMS, PATH(NMDIMS+1) + ROOT

2. (Move across.) Call GETD(DIM, IOT(DIM))

3. (Successful?) If IRTNCD $\neq 0$, then error.

4. (Move down) DIM + DIM - 1

5. (Done?) If DIM $\neq 0$, then go to 2. Otherwise, return.

If this algorithm was used with the I/O table and tree in Figure V.0.3 the final values in path would be as follows:

$$
\begin{aligned}
& \operatorname{PATH}(4)=\text { ROOT } \\
& \operatorname{PATH}(3)=1 \\
& \operatorname{PATH}(2)=7 \\
& \operatorname{PATH}(1)=9 .
\end{aligned}
$$


This is, of course, the desired path.

Now let us expand the above discussion to include subtrees. The elements of the I/O table are not restricted to be positive. The case where an element is 0 will not be covered here except to say that the routines of the EXECUTIVE effectively add 1 to all non-negative elements of the row of the $1 / 0$ table in question and then use the algorithms discussed here. Negative elements, however, warrant a more detailed discussion.

I/O table rows define subtrees of the hierarchy. The number of negative elements in any one row is the number of varying dimensions in the corresponding subtree. Since the EXECUTIVE works only with tables of two or fewer dimensions, the $1 / 0$ table vectors which are fed as input to the EXECUTIVE Traversal Algorithms can have at most two negative entries. Figure V.0.4, shows such an I/O vector. and the subtree it defines.

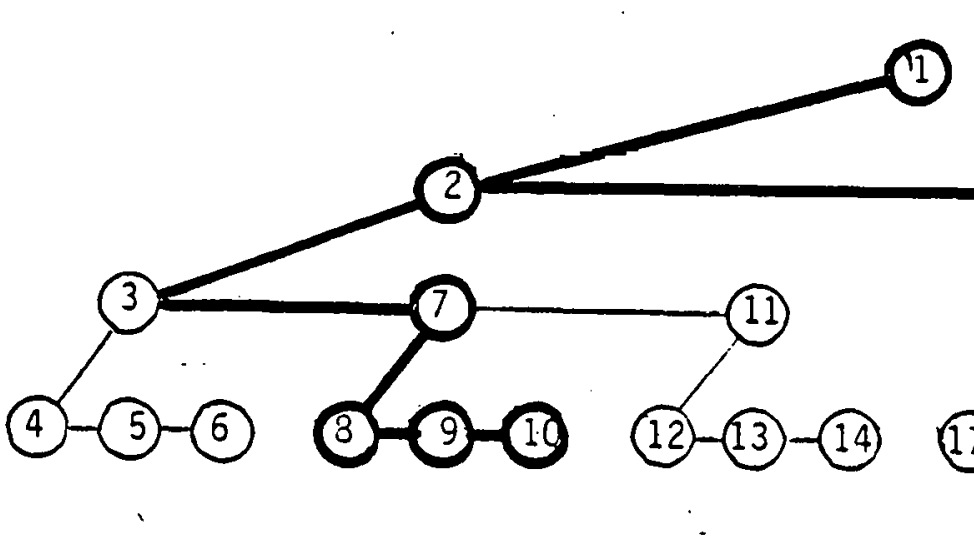

DIMENSION

Figure V.D.4. CORRESPONDENCE BETMEEN IOT AND SUB-TREE 
It is very easy to expand the path traversal algorithm into a subtree traversal algorithm, since traversing a subtree is equivalent to traversing a series of paths. The negative entries at the current $1 / 0$ table vector are initialized to one and the current path is traversed. The element corresponding to the lowest varying dimension is then incremented by one and the new path is traversed. This continues until a path does not exist in which case the element corresponding to the lowest varying dimension is reset to one and the highest varying dimension is incremented by one. Algorithm Traverse Subtree is a more detailed description of this process.

\section{Algorithm Traverse Subtree}

Given the vector IOT with NMDIMS non-zero elements, the subtree defined by this I/O table vector is traversed. ROOT is the location of the root. Algorithm Get Descendent is called as a subroutine.

1. (Initialize) $D I M+N M D I M S, P A T H(N M D I M S+1)+R O O T, T O P+0$.

2. (Varying dimension?) If IOT(DIM) $<0$, go to 9 :

3. (Move across) Call GETD(DIM, IOT(DIM))

4: (Move successful?) If IRTNCD $=4$, go to 7 .

5. (Move down) DIM + DIM - 1

6. (At bottom?) If $D I M \geq 1$, go to 2 .

7. (Done?) If TOP $=0$, return

'8. (POP.) DIM + STACK (TOP), TOP - TOP - 1, and go to 2 ,

9. (Move across 1) Call GETD(DIM, 1).

10. (Move successful?) If IRTNCD $=4$, go to 7 .

11. (Push) TOP +TOP + 1, STACK(TOP) + DIM, and go to 5 .

This algorithm is the basis of routines GDATA and RDATA. 
Thus far we have discussed the traversal of subtrees, with as many dimensions as the hierarchy itself. This capability is adequate if the user is accessing data values which all reside at dimension 1. Names, however, may reside at any of dimensions 1 through NMDIMS. Fortunately, only one line of the above algorithm needs to be changed if names rather than data are desired. If we assume that BOTTOM, an integer variable denoting the dimension from which information is to be retrieved, is passed as a parameter, then line 6 should read as follows:

6. (At bottom?) If DIM $\geq$ BOTTOM, go to 2 .

Program V.0.1. is a FORTRAN version of the above algorithm with this change.

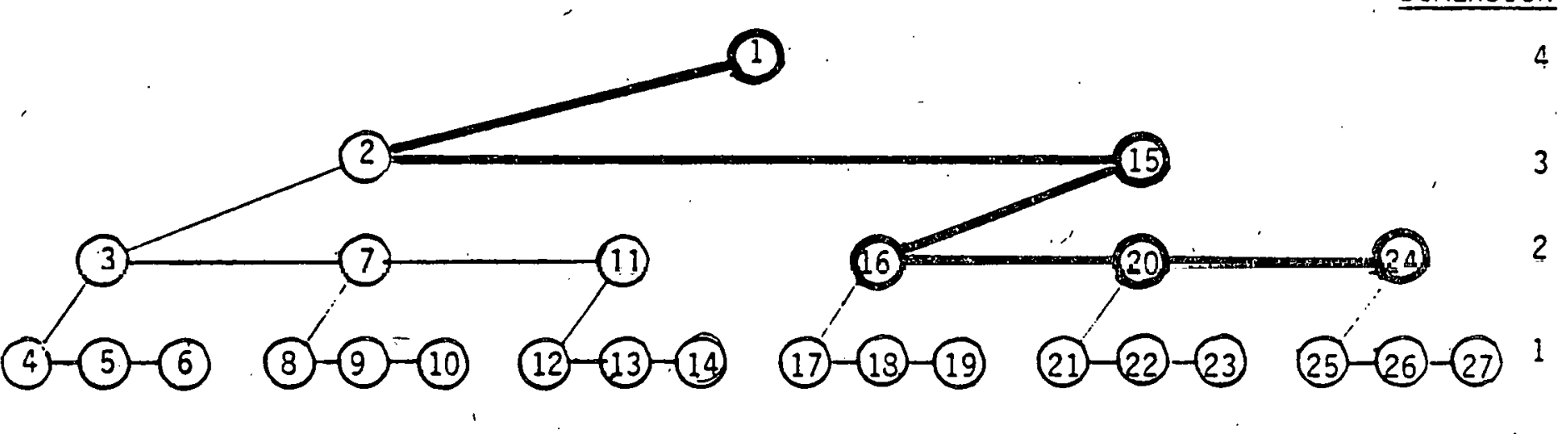

Figure V.0.5. CORRESPONDENCE BETWEEN IOT AND SUB-TREE IHHEN BOTTDM=2 
Program V.D.1. Traverse a subtree.

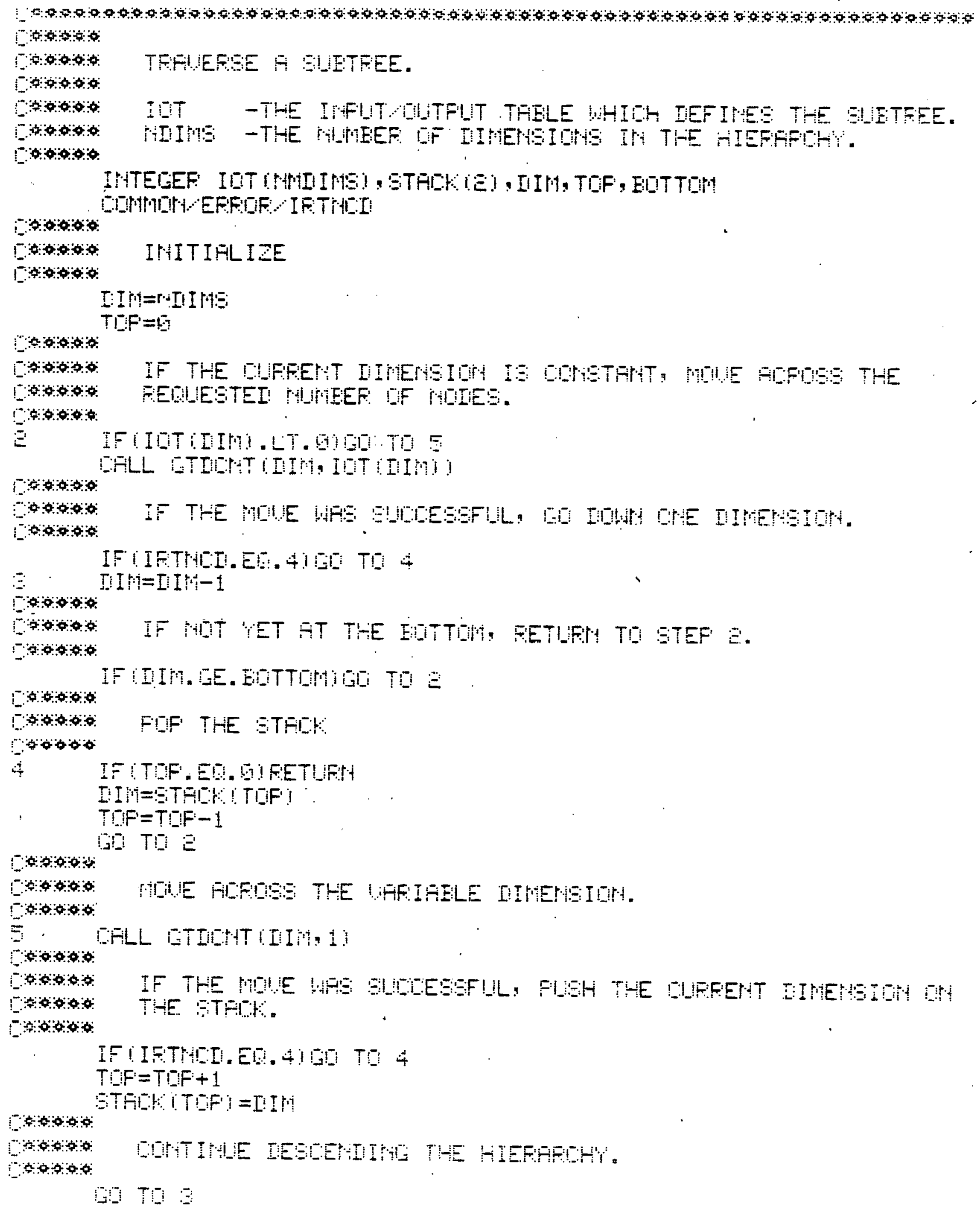


2. Updating the Hierarchy

At this point, we must explore several significant questions. First, we have discussed tree traversal in some detail but have not as yet discussed how these algorithms might be extended to perform several other needed tasks. Second, it is assumed throughout that a hierarchy already exists. How is it created in the first place? Finally, can structure be removed and if so, how? The next three subsections will address these questions.

\section{a. Accessing Data}

It was mentioned in V.0.1: that $1 / 0$ table elements could be negative, however, no significance was given to the actual values used. For two dimensional slices, a minus one means that the subtree varies along that dimension. Holding this dimension constant at one node, all data retrieved from descendents of that node of the node itself will go into one particular row of the element matrix. The rows of the elements matrix are therefore said to be varying across the associated hierarchical dimension. A minus two means that the columns of the element matrix vary across the associated hierarchical dimension. Figure V.D.6. illustrates this concept. 
DIMENSION
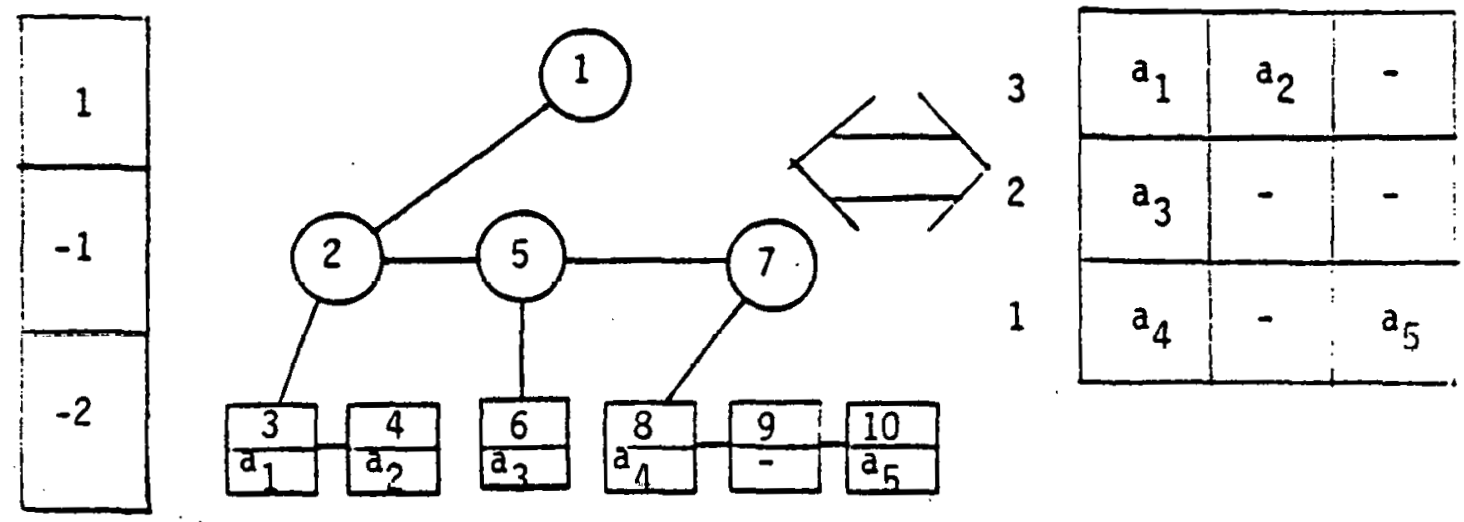

Figure V.0.6 MAPPING A TWO DIMENSIONAL

SUBTREE INTO THE ELEMENTS MATRIX

Algorithm Get Table is an extension of algorithm Traverse Subtree described in Section V.D.1. which permits data retrieval. To make this extension clear, a new utility routine, GELTDB, was written. This routine requires four parameters as input. The first is the dimension from which that data is to be retrieved and the second is the type of datum ( 1 = row name, 2 = column name, 3 = integer, 4 = floating point) to retrieve. The 
third is the variable that will receive the value. The fourth is how many words are required to hold it. GELTDB assumes that a node of the hierarchical dimension, DIM, specified by the first parameter is being pointed to be the array PATH at the time of its call.

\section{Algorithm Get Table}

This routine retrieves integers from dimension 1 of a two dimension. subtree. It uses the same parameters that algorithm Traverse Subtree uses. An additional parameter is the matrix ELMNTS with dimensions NRWSPA and NCLSPA.

1. (Initialize) DIM+NMDIMS, PATH (NMDIMS +1) +ROOT, TOP $+0, R O W+0$, COL+O.

2. (varying dimension?) If IOT(DIM)<0, go to $8 \mathrm{~A}$.

3. (move across) Call GETD(DIM,IOT(DIM))

4. (move successful?) If IRTNCD=4, go to 7 .

5. (move down) DIM-DIM-1.

6. (at bottom?) If DIM $\geq 1$, go to 2 .

6A. (get value) Call GELTDB( $1,3, \operatorname{ELMNTS}(\mathrm{ROW}, \mathrm{COL}), 1$ )

7. (done?) If TOP=0, return.

8. (pop.) DIM-STACK(TOP), TOP + TOP-1 and go to 2.

8A. (elements full?) If ( $($ IOT(DIM) $=-1)$ and (ROW=NRWSPA)) or $(($ IOT $(D I M)=-2)$ and $($ COL =NCLSPA $))$ go to $10 A$.

9. (move across 1) Call GETD(DIM,1) 
10. (move successful?) If IRTNCD $=0$, go to $10 B$.

10A. (reset elements pointer) If IOT (DIM) $=-1$ then ROW +0 . IF IOT(DIM) $=-2$ then COL +0 . Go to 7 .

10B. (increment elements pointer) If IOT (DIM) $=-1$ then ROW ROW + I. If $\operatorname{IOT}(D I M)=-2$ then $\mathrm{COL}+\mathrm{COL}+1$.

11. (push) TOP $+T O P+1, \operatorname{STACK}(T O P)+D I M$ and go to 5 .

When names are to be retrieved, the IOT input to the retrieval algorithm must first be transformed using name conditionality. This transformation can be found in function GIONT. There are two steps involved. First, if row names are desired, we must locate the -1 element of the $1 / 0$ vector, set BOTTOM to the hierarchical dimension which corresponds to this negative I/0 element, and get the name conditionality table row corresponding to dimension BOTTOM. We next do the following for al1 IOT(i), BOTTOM<i<NMDIMS.

1. If $(\operatorname{IOT}(i)>0)$ and $(\operatorname{NC}(i)=0), \operatorname{IONT}(i)+1$.

2. If $(\operatorname{IOT}(i)>0)$ and $(\operatorname{NC}(i)=1), \operatorname{IONT}(i)+\operatorname{IOT}(i)$.

3. If $\operatorname{IOT}(i)=0$ then $\operatorname{IONT}(i) \leftarrow 0$.

4. If $\operatorname{IOT}(i)<0$ then $\operatorname{IONT}(i)+1$.

Two further changes must be made in algorithm Get Table. First as. explained in Section V.D.1, line 6 must read:

6. (at bottom?) If DIM $>B O T T O M$ go to 2 . Second, line $10 \mathrm{C}$ must be addad:

10C. (get name) If (NAME) and (IOT(DIM) $=-1$ ) then GELTDB (BOTTOM, 1 , ROWNAM(I), NWPARN)

where NAME is a logical variable indicating that names are to be retrieved. The reader should study subroutine GDATA with some care to see how the concepts discussed so far have been implemented. 


\section{b. Adding Structure}

When replacing a table, the same traversal algorithm is used. One potential difficulty can arise however, in that all or part of the. subtree to be traversed might not exist.

The MVTORT ('move to the right') subroutine provides a solution to this problem. "States simply, the algorithm moves across any number of occurrences, say $N$, of nodes at a certain hierarchical dimension, say DIM, to the right. The exact number of moves and particular dimension are specified in the subroutine call. The power of this routine is that if there are not enough occurrences of the nodes to make the move the algorithm will automatically insert occurrences until the move can be made.

The following condition must be satisfied prior to any call to MVTORT.

$$
\operatorname{PATH}(D I M+1) \neq \Lambda
$$

According to the above condition a node at dimension DIM, does not have to be pointed to. If such a pointer does exist, MVTORT will move the pointer $n$ occurrences to the right. If such a pointer does' not exist, the $N^{\text {th }}$ child of the node indicated by PATH(UIM+1) will be accessed. 
Another important property of this routine is that it may be substituted directly for GTDCNT in the Get Table algorithm. The resulting algorithm will traverse the desired subtree, adding structure when necessary. If, in addition, the utility routine, PELTDB, which places elements in the data base, is substituted for GELTDB, the new algorithm will replace the entire contents of the elements matrix in the data base. This is precisely what algorithm Replace Table does.

\section{Algorithm Replace Table}

This routine replaces the values of the elements matrix in dimension 1 of the hierarchy according to the definition in IOT. It calls utility routines MVTORT and PELTDB described above.

1. (Initialize) DIMANMDIMS, PATH(NMDIMS+1) +ROOT, TOP +0, ROW +0, COL +0 .

2. (varying dimension?) If $\operatorname{IOT}(D I M)=0$, go to $8 \mathrm{~A}$.

3. (move across) Call MVTORT(DIM,IOT(DIM))

5. (move down) DIM+DIM-1

6. (at bottom?) If $D I M \geq 1$, go to 2 .

6a. (placevalue) Call $\operatorname{PELTDB}(1,3, \operatorname{ELMNTS}(R O W, C O L), 1)$

7. (done?) If TOP=0, return.

8. (pOP) DIM STACK (TOP), TOP +TOP-1 and go to 2.

8a. (current dimension done?) If $((\operatorname{IOT}(D I M)=-1)$ and (ROW =NRWSPA $)$ ) or $((\operatorname{IOT}(D I M)=-2)$ and $(C O L=N C L S P A))$ to to 9 .

8b. (reset ELMNTS pointer) If IOT(DIM) $=-1$, then ROW 0 . If IOT(DIM) $=-2$, then COL +0 . Go to 7 . 


\section{9. (move across 1) Call MVTORT(DIM,1) \\ 10. (increment elements pointer) If $\operatorname{IOT}(D I M)=-1$ then ROW + ROW +1 . If IOT(DIM) $=-2$ then $\mathrm{COL}+\mathrm{COL}+1$ \\ i1. (push) TOP $+T O P+1$. STACK(TOP) $+D I M$ and to to 5 .}

Algorithm Replace Table is essentially the algorithm used in RDATA. Some extensions have been added to allow for the replacement of names. Another extension called "Garbage Collection" is discussed below.

\section{c. Removing Structure}

In the preceding section we described how to modify the Get Table algorithm so that the entire elements matrix can be replaced in a subtree all of whose structure need not exist. As mentioned in Section V.B, some of the elements matrix values may be default values. In certain cases, these values and the tree structure necessary to hold them should not be part of the hierarchy. To make those cases clear, the following definition is made.

Definition. The tree is said to be in standard tree order if there does not exist a node such that

1) it holds no data values

2) it has no descendents

3) it has no younger siblings 
Algorithm Garbage Collection below is used to maintain standard tree order in the hierarchical tree. It uses an inverse pre-order traversal which is defined as follows:

1. Traverse rightmost subtree in inverse pre-order.

2. Traverse the second rightmost subtree in inverse pre-order and continue in this manner until all subtrees have been traversed in inverse pre-order.

3. Visit the root. $\rightarrow$

The nodes of the tree bilow are numbered in inverse tree order.

DIMENSION

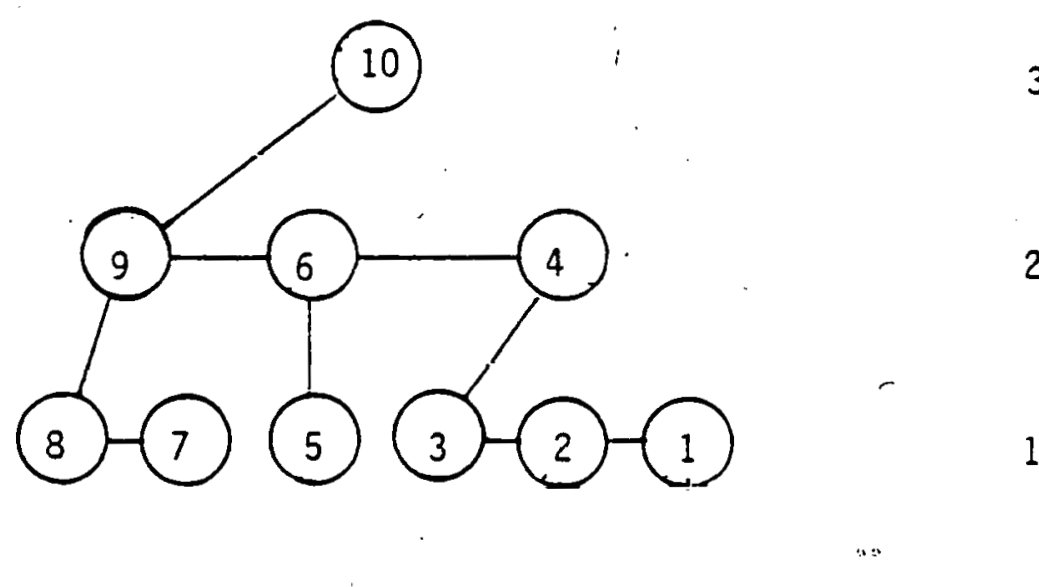

Figure V.D.7. NODES IN INVERSE PRE-ORDER

To accomplish this traversal, a new utility rnutine is necessary. Notice that an inverse pre-order Traversal steps agairst the arrows generated by the preorder. If we wished to go from say node 1 to node 2, the Get 
Descendent algorithm can no longer bè used by itself. However it can be used in conjunction with the $\operatorname{FSTDEC}(X)$ operation discussed in Section V.D.1

\section{Algorithm Garbage Collection}

Four input parameters are required for this algorithm. CURDIM is the dimension at which to start. NXTDIM is either the next highest dimension above CURDIM which is varying in the current subtree or NMDIMS+l if no such dimension exists. CURPOS is the position of the current node at dimension CURDIM relative to its father and IOT is the current I/0 vector.

Subroutine GTDFST, which performs a FSTDEC(PATH(CURDIM+1)), is called during this procedure. In the tree above, $\operatorname{PATH}(1)$ points to node 1 , then GTDFST( 1 ) would return a pointer to node 3 in PATH(CURDIM). GTDCNT is also used. REMTRE removes the branch currently pointed to at the dimension which is passed as its only argument. CHKNUL returns true if and only if the node currently pointed to at the dimension which is its argument has any data. The algorithm is:

1) (younger siblings?) Call GTDCNT( CURDIM,1). If IRTNCD=0, then return.

2) (descendents?) If CURDIM $=1$ go to 3 . Otherwisc CALL GTDFST (CURDIM-1). If IRTNCD $=0$, then return.

3). (data?) If not, CHKNUL(CURDIM) then return.

4) (remove) Call REMTRE(CURDIM)

5) (dimension done?) If CURPOS>1 then go to 9.

6) (move up 1 dimension) CURDIM+CURDIM+1

7) (done?) If CURDIM=NXTDIM then return.

8) (get position) CURPOS+IOT(CURDIM) and go to 1 .

9) (get next oldest sibling) CURPOS+CURPOS-1. CALL GTDFST(CURDIM), CALL GTDCNT (CURDIM,CURPOS-1) and go to 2. 
If this algorithm is called with CURDIM=DIM and NXTOIM=STACK(TOP). in the Replace Table routine described in the preceding section just prior to the popping of the stack, standard tree order will be preserved. 


\section{E. Appendix}

Data Base Definition - five trees sharing a common root

The root

-50. TEL HE:

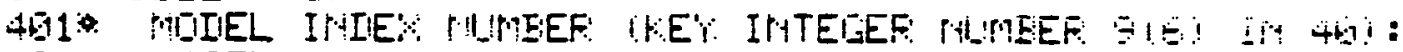

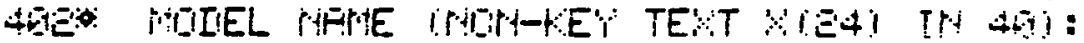

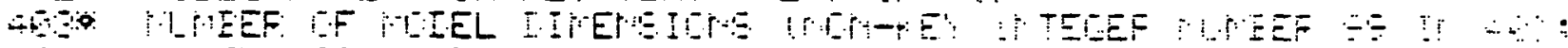

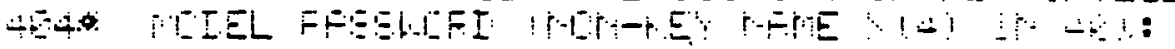

Tree \#1 - Input/output table

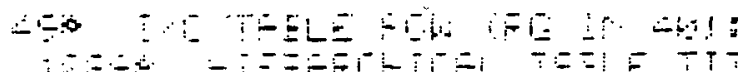

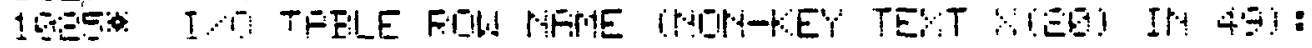

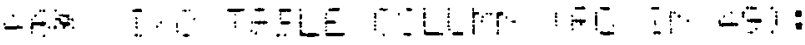

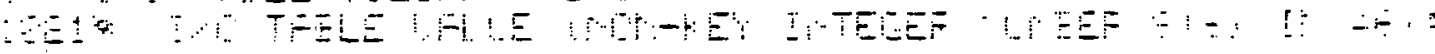

Tree \#2 - Labeled common tables

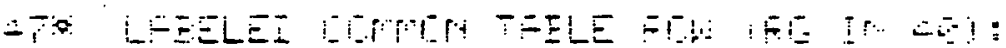

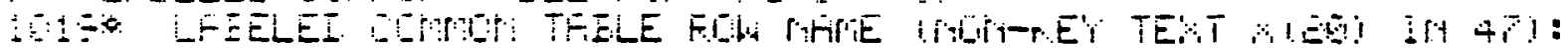

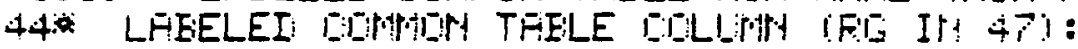

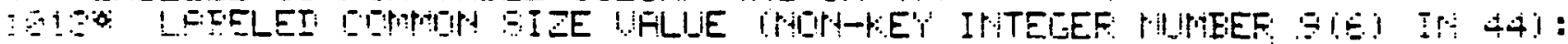

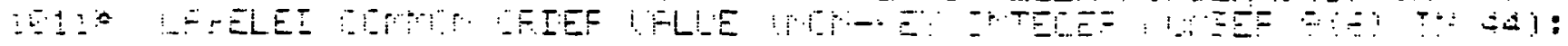

Tree \#3 - Name conditionality table

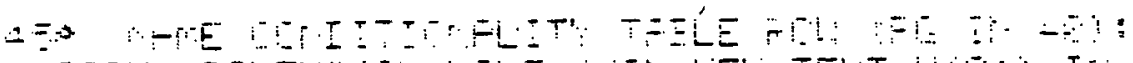

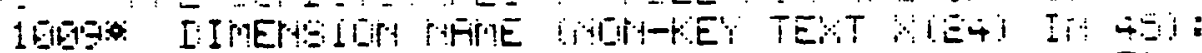

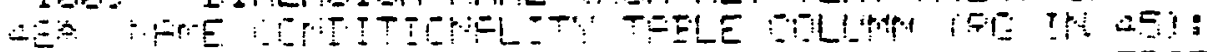

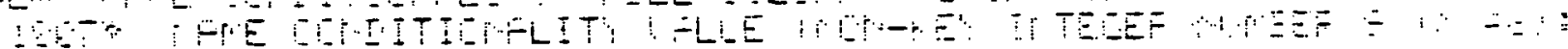

Tree \#4 - Storage conditionality table

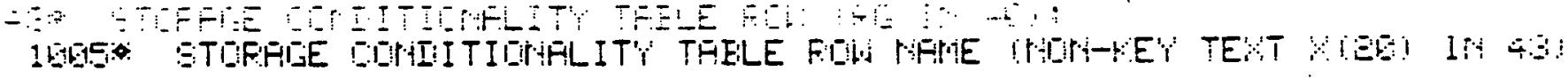
:

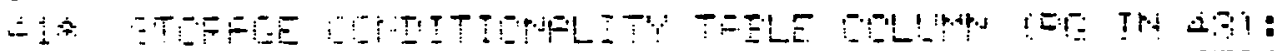

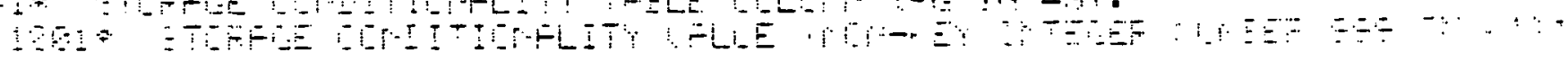

Tree \#5 - The hierarchical tree

50 -

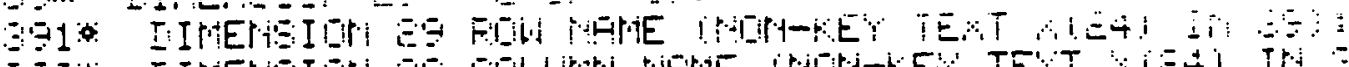

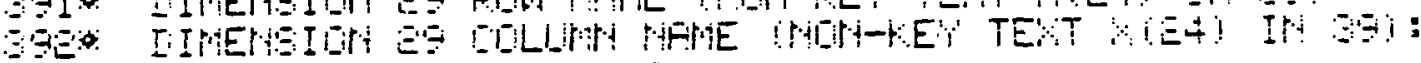

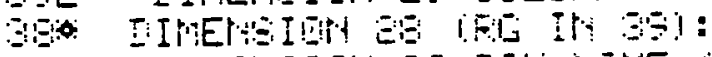

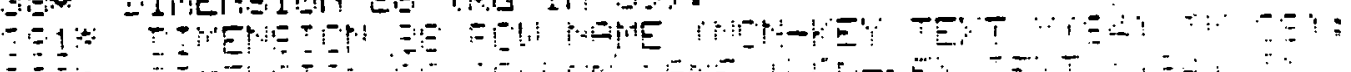

Sa 


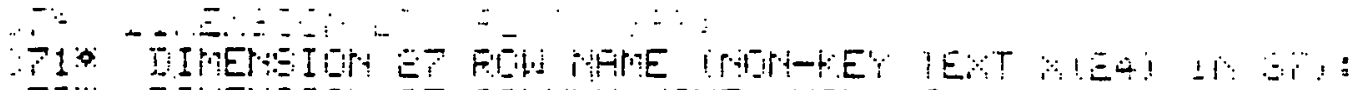

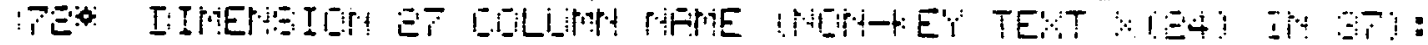

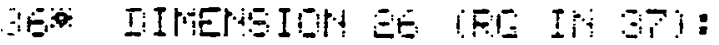

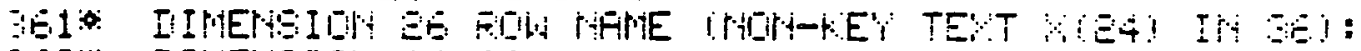

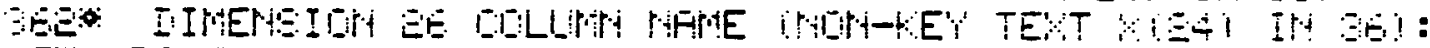

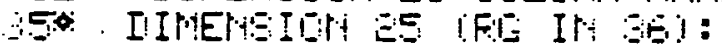

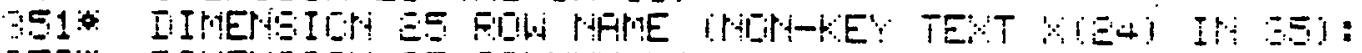

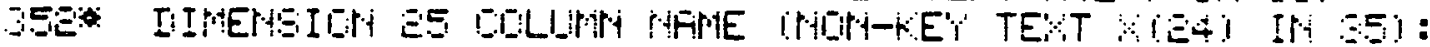

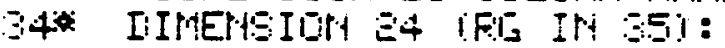

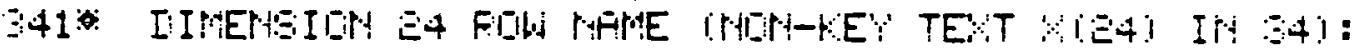

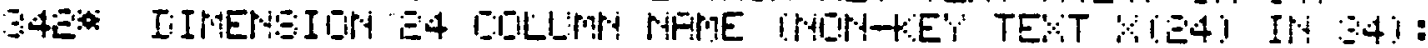
3\% IITHEISIDH E IFIE IH $341:$

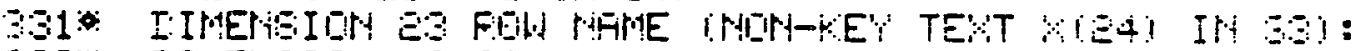

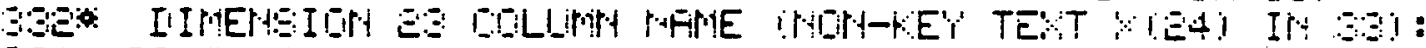

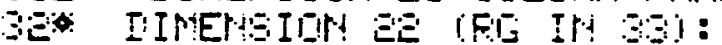

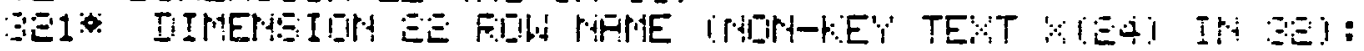

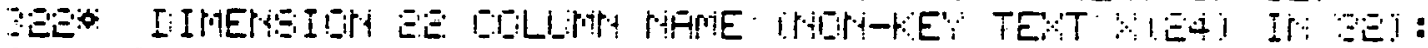

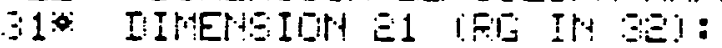

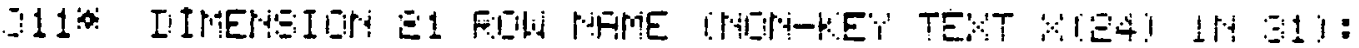

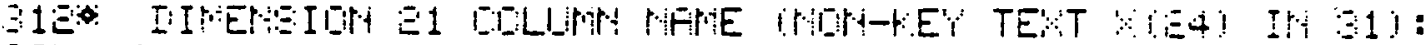

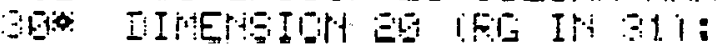

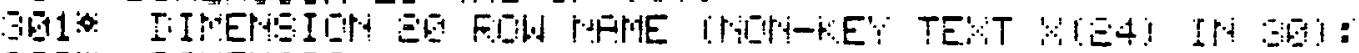

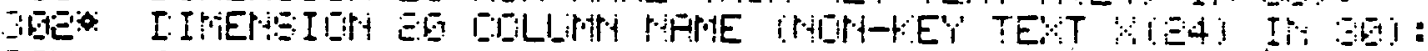

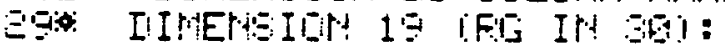

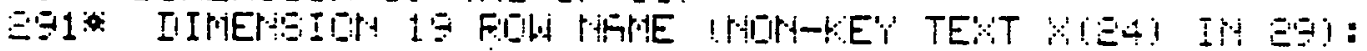

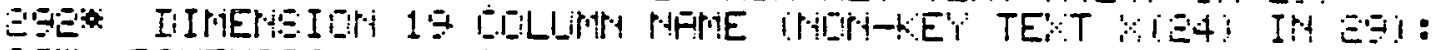

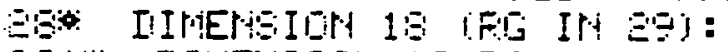

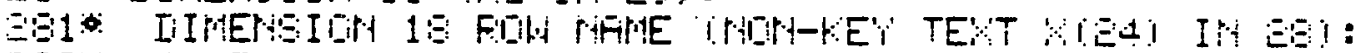

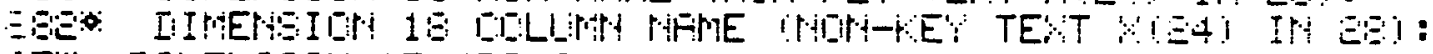

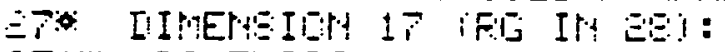

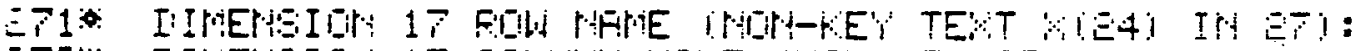

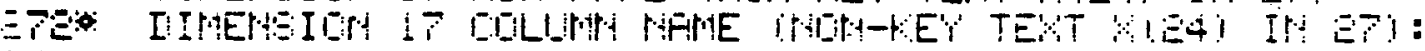

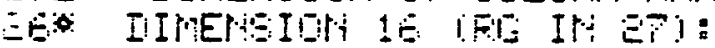

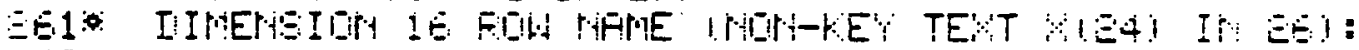

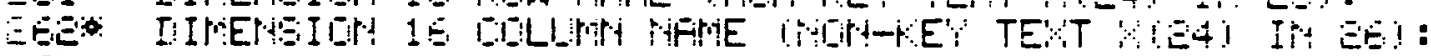

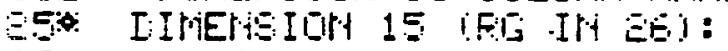

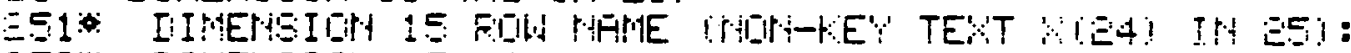

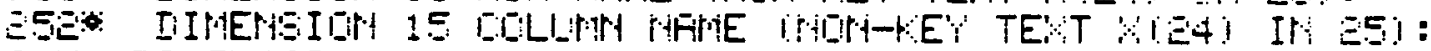

Eto IIPEHSILI! 14 IFI IH EEI:

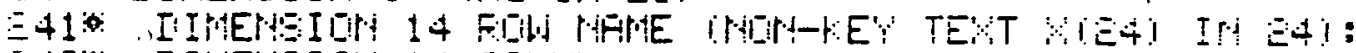

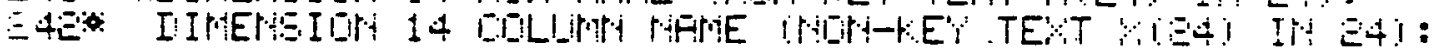

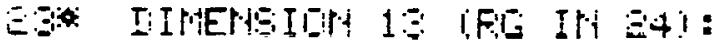

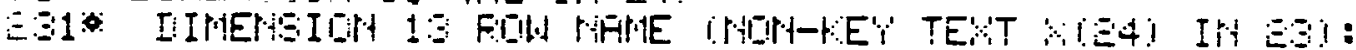

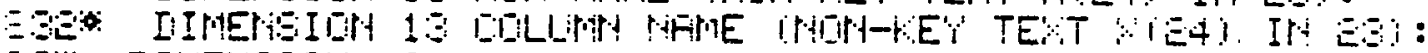

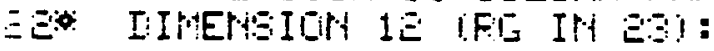

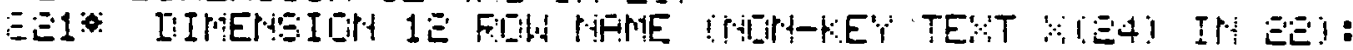

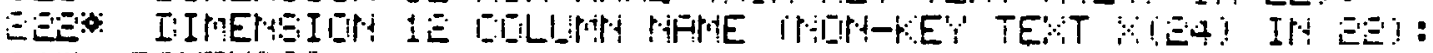

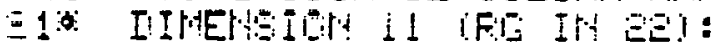

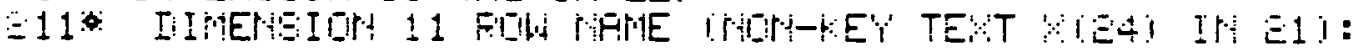

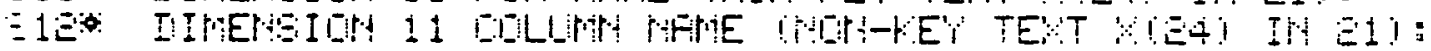

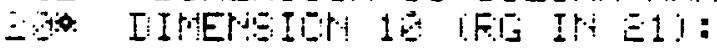

E E 


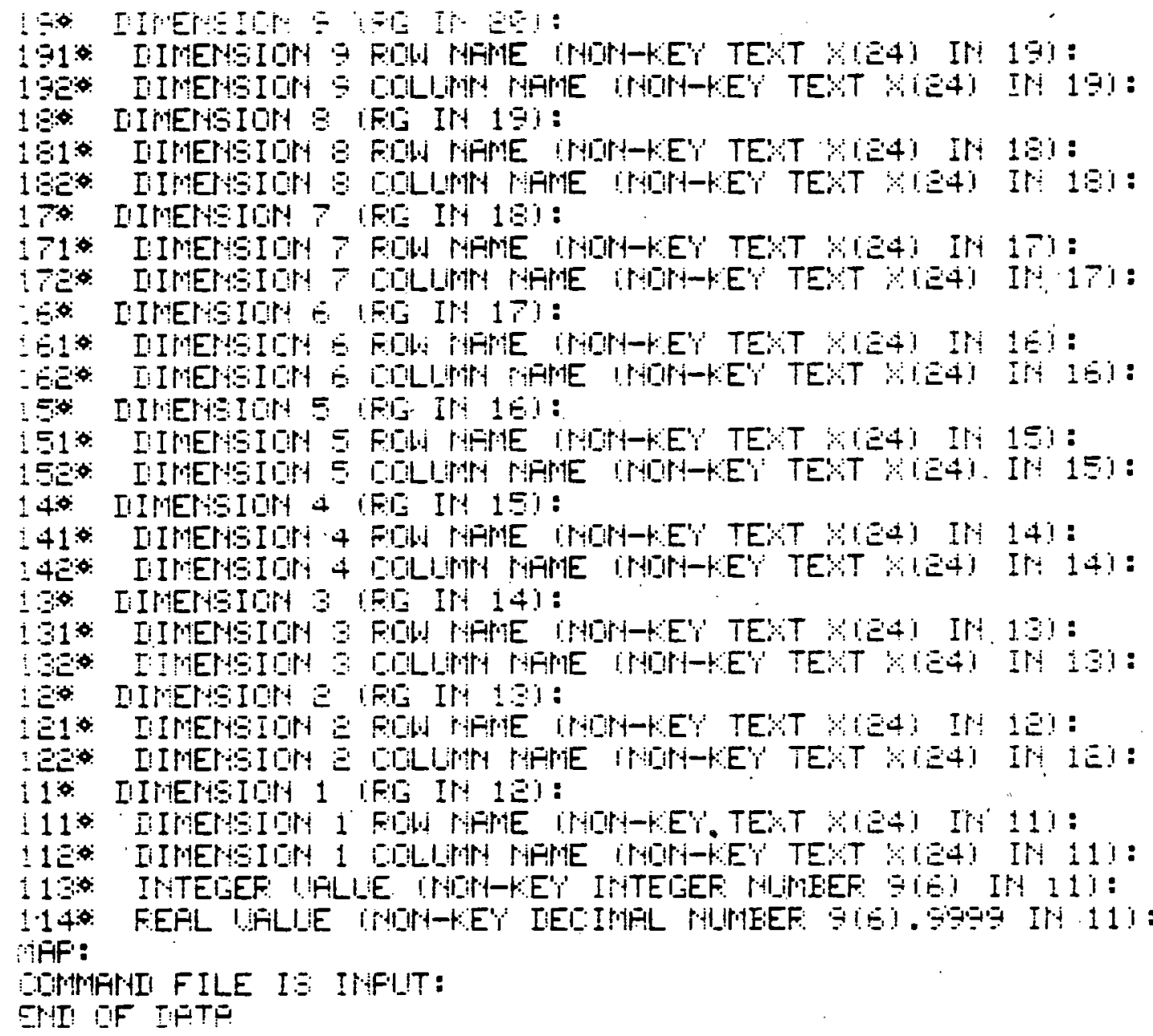


F. Bibliography

Procedural Language Interface:

System 2000 Extended Procedural Language Interface, \#UM1.

(Houston: MRI Publications, 12/76)

\section{Parsing:}

Wirth, Nicholas, Algorithms + Data Structures = Programs, (Englewood Cliffs, New Jersey: Prentice-Hall, Inc., 1976)

Tree Traversal:

Knuth, D.E., The Art of Computer Programming, Volume 1, (Menlo Park, California: Addison-Wesley Publishing Company, 2/75) 
CHAPTER VI

DATA TRANSFER SYSTEM -- PROGRAMMERS GUIDE

\section{A. INTRODUCTION}

The data transfer system is a collection of six FORTRAN subroutines: SOFTIN, SOFTOU, FILL, STOR, SFILL, and SSTOR. The first part of this chapter will outline the major algorithms in detail for each of these six subroutines. In these discussions we will define the required user input data, internal program variables, notation used to implement the algorithms and data structures, and present detailed flow charts. We will conclude with a brief identification of some utility subroutines and machine dependencies and give some heloful hints to those who will be continuing to develop the software. These tips include how to use the write flags provided for detugging during software development.

The methodology underlying the Data Transfer System was discussed in considerable detail in Chapter III. This chapter will refer liberally back to that discussion.

B. THE SOFTIN ALGORITHM

1. Overview

Referring to Figure II.C.1, the purpose of the SOFTIN algorithm is to process the hierarchical data tree and to store the values at 
the terminal branches of the tree in a single vector in a form accessible to the model logic. In the figure, this corresponds to Step 1. However, when we speak of SOFTIN, we are referring to Step 1 in the figure or to the process by which we simply inspect an "intermediate" $\underline{d}$ - vector without reconstructing the tree. Thus, SOFTIN refers to the following procedures:

1.) Reading an existing d-vector, together with offset and system table information, into active storage, or

2.) Converting from the hierarchical tree representation of the data base to the d-vector.

Because it is simpler and less often used, we will discuss option 1.) first. Under this option, we simply read labeled common LBLO from the binary file.FTOQ3 which is assumed to be of the form LElITDT, $A(2), \ldots, A(L E N T O T)$. Here LENTOT is a 4 byte. integer work and $A(I)$ is read as unformatted 4 byte words with $A(I)$ the first word in LBLO. This option is provided as a restart capability since labelled, common LBLO contains all the information required to run a model. suD-

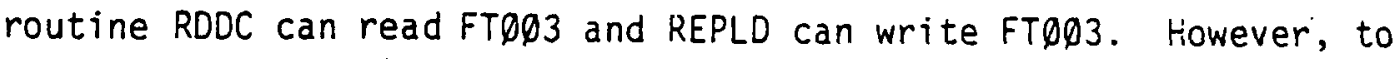
replace a model data base in the System 2000 data base, the SOFTOU algorithm requires the same file FTOO2 as the one assumed in alternative. 2.) The capability to regenerate the hierarchical tree from the d-vector and the offsets alone has not yet been implemented. Thus, at the present, option 2.) must be run to execute the model and save the results in the data base. -- option 2.) can be run only for special, limited purposes.

The second option is the algorithm which reads the model information from the System 2000 unlead file, FT002 containing one complete model data base in System 2000 value string format (see Siystem 2000 manual). To create 
the System 2000 unload file, we use the System 2000 command: UNLOAD BY C40, C40 WHERE C40 EQ $X$;. Here $X$ is symbolic for a positive integer constant denoting the model data base number.

Option 2.) consists of three separate algorithms:

(1) Build the system tables (BLDTBL): Read the input system tables from the top of file and generate the system tables.

(2) Build the offset's (BLDOFF): Generate the three dimensional array of offsets, $\operatorname{IOFF}(I, J, K J S)$. This algorithm makes, one pass through the FRM2K file, starting from the character position in FRM2K where the first algorithm left off, and continues until it detects the terminal character string "FINI".

(3) Build the data vector (BLDD): First rewind FRM2K and skip to the beginning of the hierarchy. Then make a second pass through the model data, transferring values into the RD vector in positions consistent with the offsets in IOFF.

An overview of option 2.) of the SOFTIN algorithm is, flow charted in Figures VI.B.1 - VI.B.3. We will discuss these three steps of SOFTIN in some detail below after first discussing the System 2000 unload file conventions that pertain.

\section{Data Storage}

In order to understand the operation of SOFTIN, one must understand the conventions under which the data is stored in the CNiS. Figure VI.B.4 illustrates the genernl storage layout. The tree is drawn according to the node/branch conventions introduced in Chapter II. In the figure, each circle is a shorthand representation of a large number of nodes at each level rather than a single node. Each solid rectangle represents a set of names or values associated with the nodes at each 


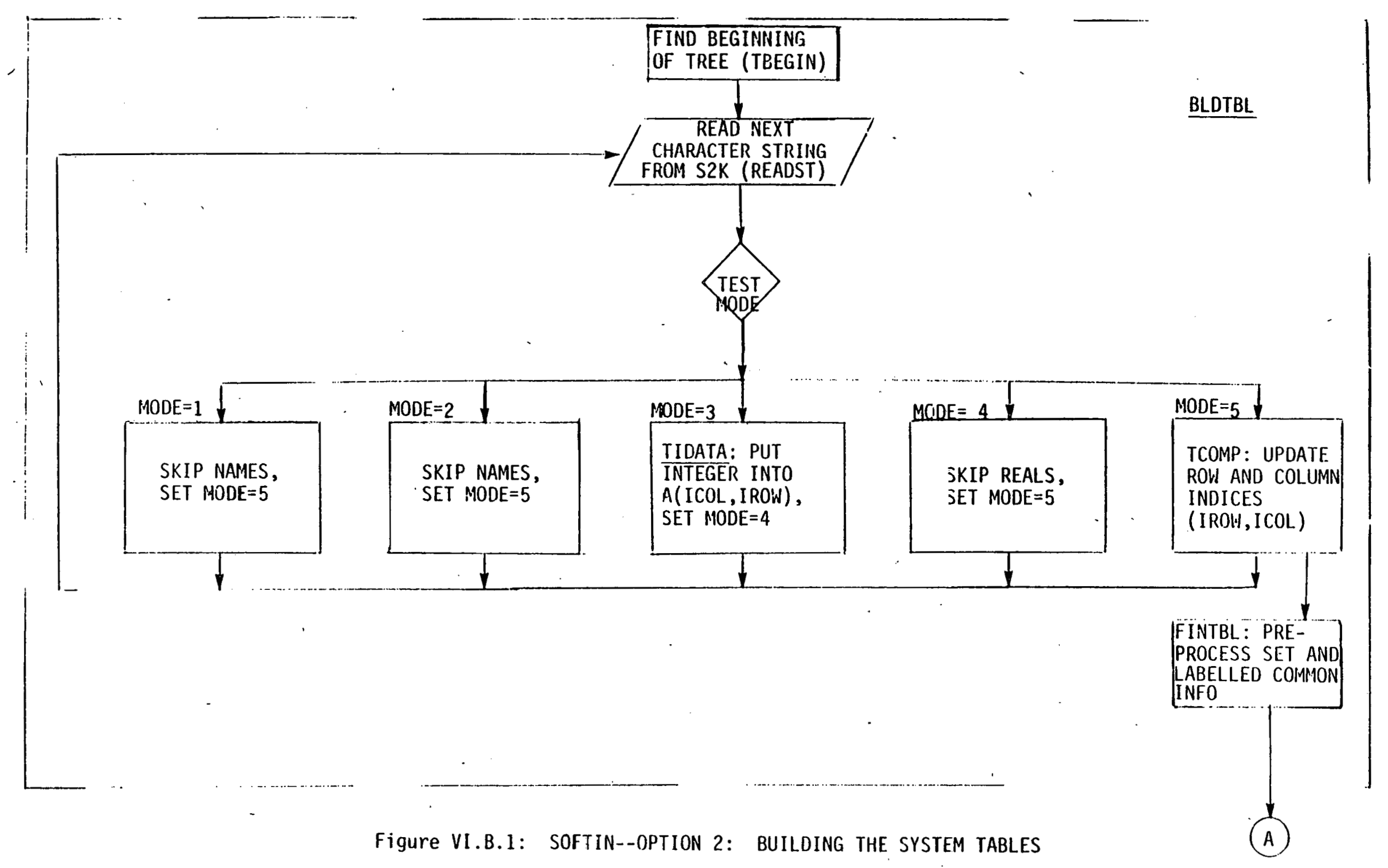




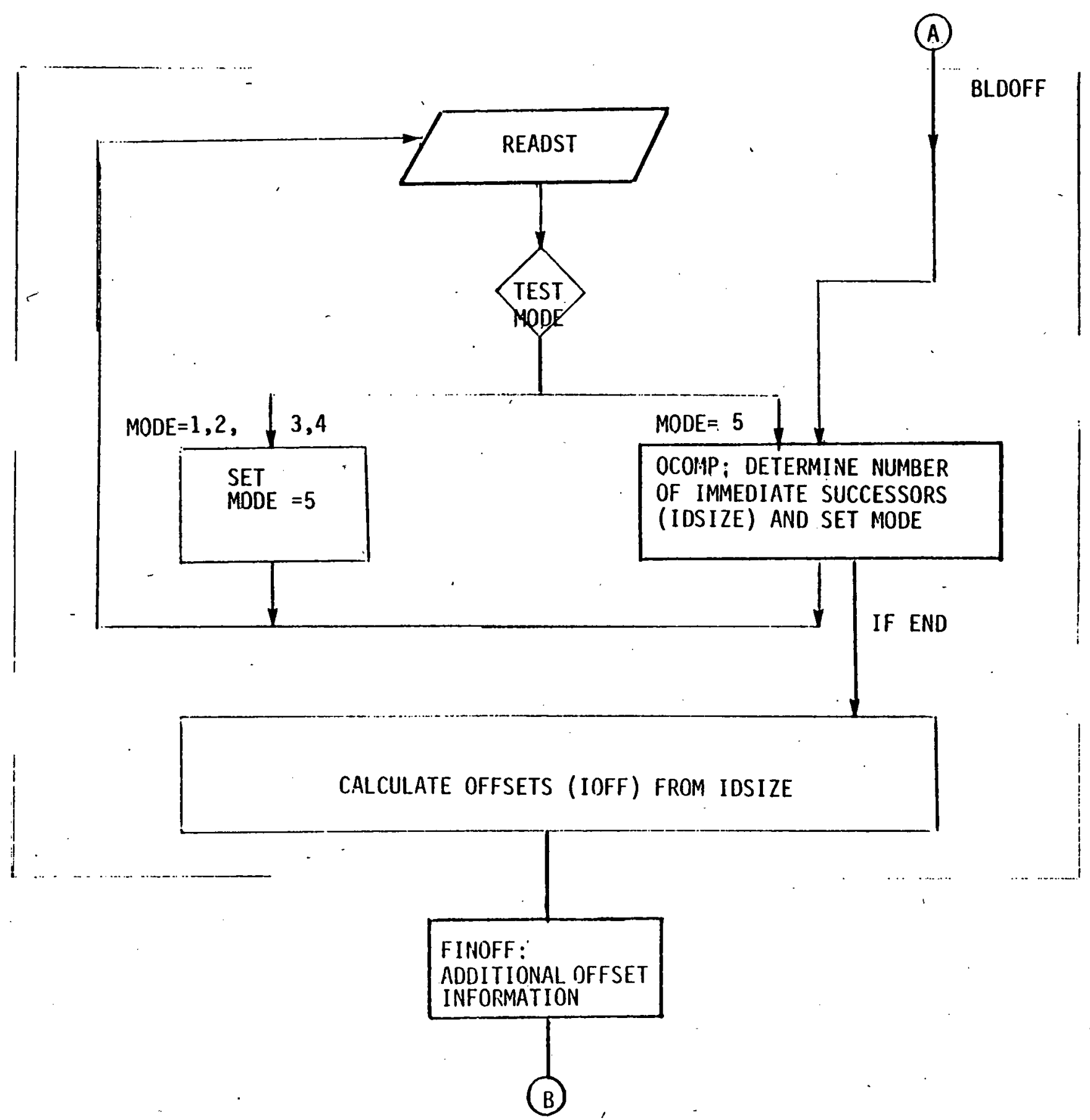

Figure VI.B.2 SOFTIN--OPTION 2: BUILDING THE OFFSETS 


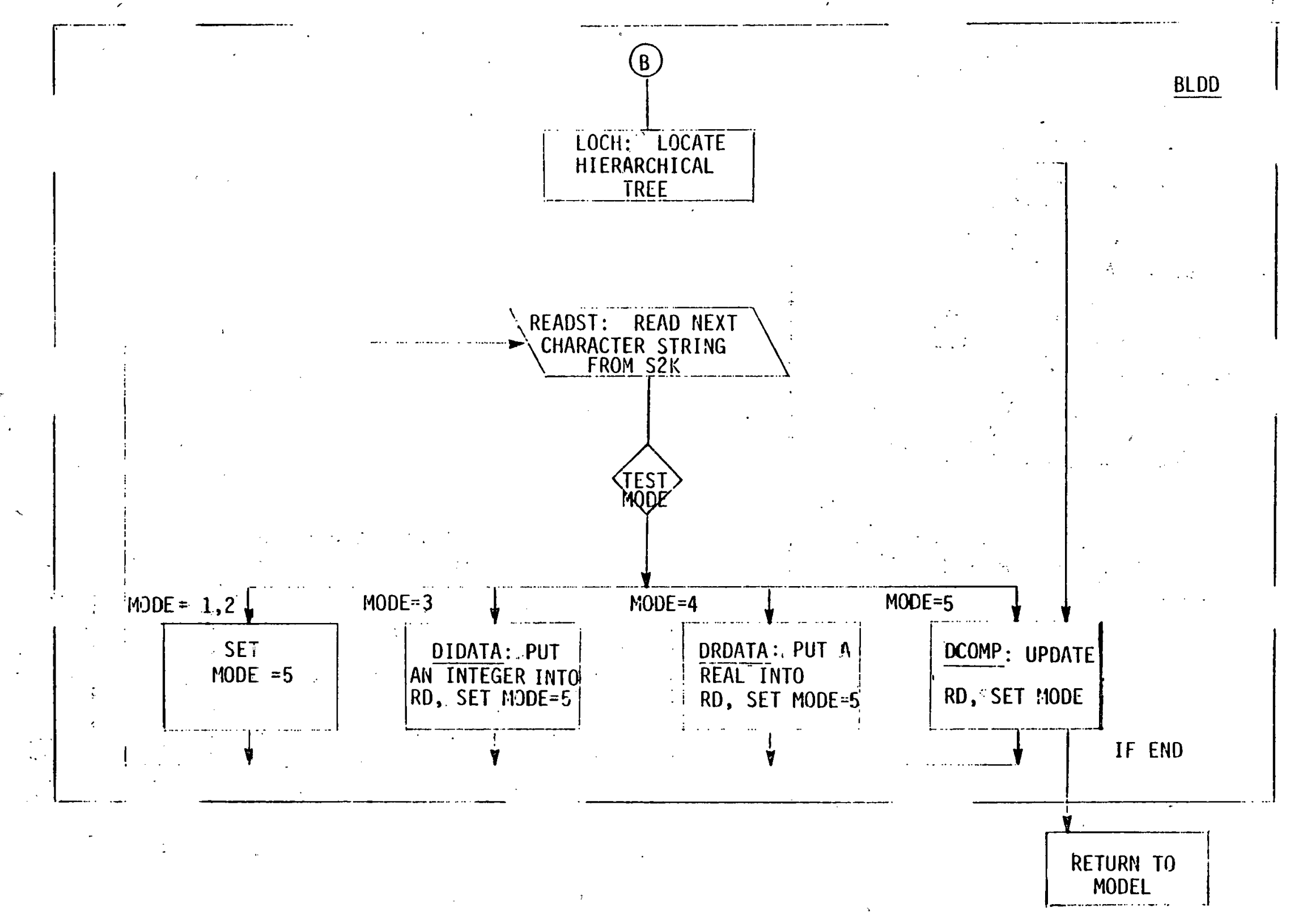

Figure VI.B.3: SOFTIN--OPTION 2: BUILDING TEIE RD YECTOR 


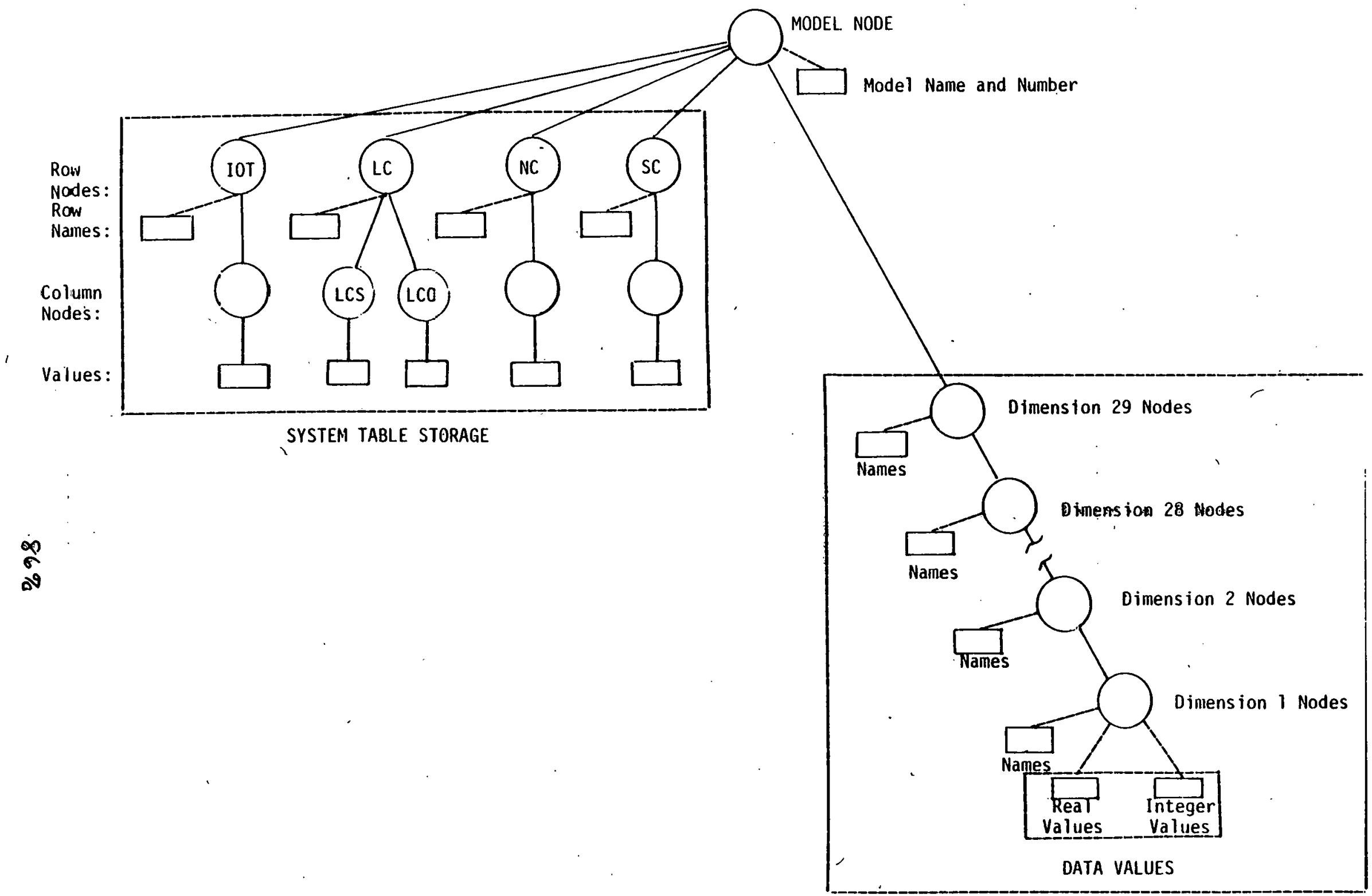

VI.B.4: CMS DATA BASE STRU::TURT

HIERARCHICAL DATA STORAGE 
particular level. In a particular model, the user must define the number of nodes at each level. The distinction made between system table storage and hierarchical data storage made in Figure VIB.4 is important since both the Data Transfer System and the Executive use different algorithms to access the system tables than to access the hierarchical data tables.

As illustrated in Figure VI.B.4, the system table values (and their associated names) are stored separately from the rest of the model information. For each system table one node is defined for each row in the table. Descendant from each of these row nodes is a node for each column in the table, associated with which is the corresponding value in the table. Row names are associated with the row-nodes. This scheme of storage is illustrated for a $2 \times 2$ system table in Figure VI.B.5. All the system tables are stored this way except the labelled common size and labelled common order tables which. share the same row nodes.

\section{Reading the System Tables (BLDTBL/FINTBL)}

Because the algorithm we use to read the system tables is similar to those we use to set the offsets in BLDOFF, we shall defer our detailed discussions to that section. We will, however, summarize the calculations that are made here and summarize the definitions of the pertinent system tables.

Labelled Common Size Table. The labelled common size table contains the basic information about how many individual components can occur along each dimension in each labelled common. Implicit in this 
TABLE

\begin{tabular}{|c|c|c|}
\hline \multicolumn{3}{|c|}{ Column } \\
\hline & 1 & 2 \\
\hline Row Name 1 & $e_{11}$ & $e_{12}$ \\
\hline Row Name 2 & $\mathrm{e}_{21}$ & $\mathrm{e}_{22}$ \\
\hline
\end{tabular}

STORAGE

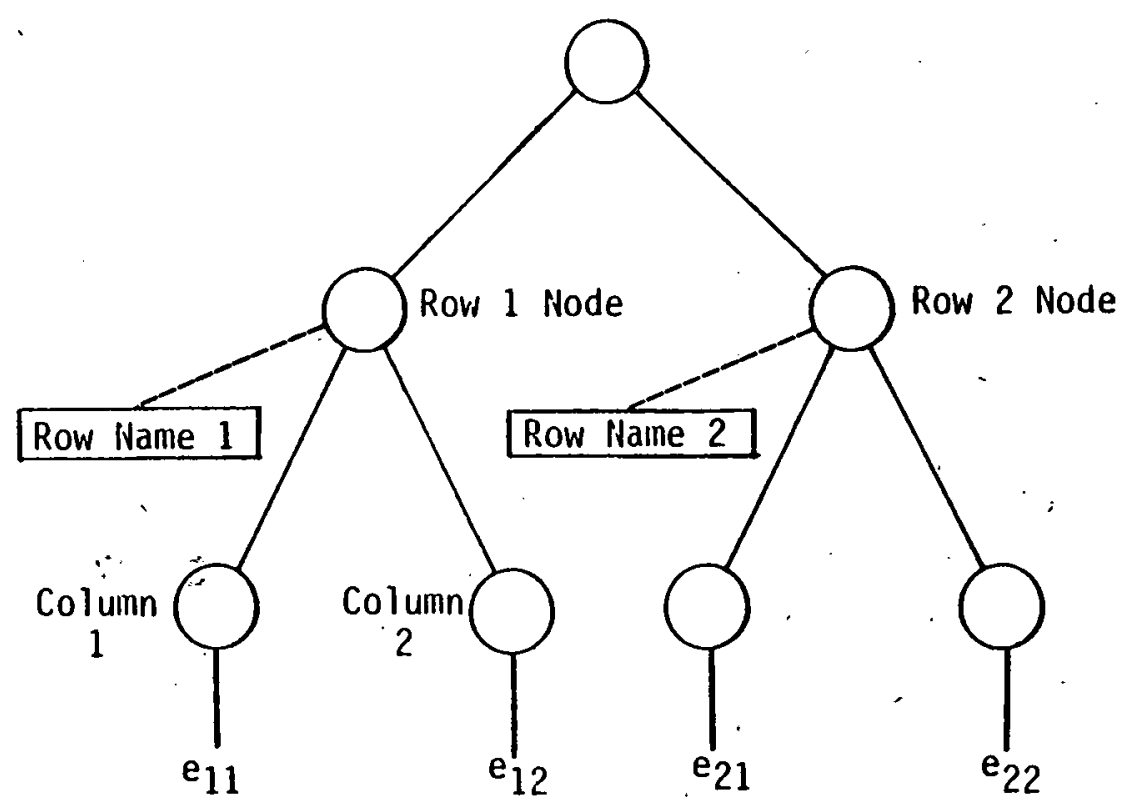

Figure VIT.B.5: SYSTEM TABLE STORAGE 
table is the information as to how many dimensions vary within each labelled common. We will store this information in the matrix $\operatorname{LCS}(I, J)$ where I runs over all the dimensions of the model $I=1, \ldots$, NDIM and $J$ runs over all the labelled commons of the model $\mathrm{J}=1, \ldots, \mathrm{NLC}$. If $\operatorname{LCS}(I, J)$ is strictly positive, then we know that there are $\operatorname{LCS}(I, J)$ elements along the I-th dimension in the J-th labelled common. We , will say that $\operatorname{LCS}(I, J)$ is the size of dimension I in labelled common $J$. If $\operatorname{LCS}(I, J)=0$, we know that dimension $I$ is not in the $j$ th labelled cormon.

Labelled Common Order Table. The labelled common order table gives the order in which the dimensions are arranged within each labelled common. The labelled common order table is denoted LCO(I,J), where $I$ runs over all the dimensions and $\mathrm{J}$ runs over all the labelled commons, Given the J-th labelled common, we will discuss the contents of the vector

$$
\begin{aligned}
& \operatorname{LCO}(i, J) \\
& \operatorname{LCO}(2, J) \\
& \vdots \\
& \operatorname{LCO}(N D I M, J) .
\end{aligned}
$$

The fastest varying dimension in the $\mathrm{J}$-th labelled common is indexed $l$ in the appropriate position. The second fastest varying dimension in the $\mathrm{J}$-th label led common is indexed 2 in the appropriate position, and so forth. Thus, the $J^{\text {th }}$ column of the labelled common order table 
contains the hierarchical order of the dimensions within the $\mathrm{J}$-th labelled common itself, which may be different than the order of the dimensions in the hierarchical data tree.

The dimensions that appear in the J-th column of the labelled common order table and have positive indices are those that must be varied to fill the J-th labelled common. However, the numerical values that are placed into the $\mathrm{J}$-th labelled common may be conditional on fixed values of other dimensions. This dependency must also be specified in the labelled common order table. If the $\mathrm{J}$-th labelled common is conditional on the value of the I-th dimension, a negative number will appear in $L C O(I, J)$. The negative numbers placed into the $\mathrm{J}$-th column must appear in a particular order -- the dimensions lower in the dimensional hierarchý must be "less negative" than those higher in the hierarchy.

LCPS(I.J.): Labelled Common Problem Structure. This table is created by BCDTBL' and used by SOFTIN. The J-th column corresponds to the $\mathrm{J}$-th labelled common, $\mathrm{J}=1, \ldots, \mathrm{NLC}$. Each of the rows corresponds to a different property of the various labelled commons.

Row 1: The J-th element in row 1 contains the binary index of the set from which the J-th labelled common is filled.

Row 2: The $\mathrm{J}$-th element in row 2 contains the number of free dimensions over which the J-th labelled common varies.

Row 3: The length of the $\mathrm{J}$-th labelled common. The $\mathrm{J}$-th clement in row 3 contains the product of the sizes of the dimensions over which the J-th labelled common varies. The sizes are taken from the $\mathrm{J}$-th column of the labelled commón size table. 
Row 4: The J-th element in Row 4 contains the number 1 plus the sum of the lengths of labelled commons $1,2, \ldots, \mathrm{J}-1, \mathrm{i} . \mathrm{e}$. , the position of the first word in the $J$-th labelled common.

Row 5: The J-th element in Row 5 contains the number of the set which labelled-common $\mathrm{J}$ belongs to after the sets are ordered by binary indices.

$\operatorname{ISPS}(1, J)=$ Set Problem Structure. This table is also created by BLDTBL. The $\mathrm{J}$-th column corresponds to the $\mathrm{J}$-th set, $\mathrm{J}=1, \ldots$,NSETS. Each of the rows corresponds to a differént property of the various sets.

Row 1: The J-th element in Row 1 contains the binary index of the J-th set. Furthermore, the elements in Row 1 are strictly increasing.

Row 2: The J-th element in Row 2 contains the number of dimensions in the J-th s'et.

Row 3: The J-th element in Row 3 contains the hierarchical index of the highest level dimension in the $\mathrm{j}$-th set (attribute is the lowest level dimension).

Row 4: The J-th element in Row 4 contains the number of "dependent" branches for the highest level dimension in the J-th set (computed in BLDOFF/DCOMP)

Row 5: The J-th element in the 5th row of ISPS is the index in the $\mathrm{RD}$-vector where the first element of the J-th set will be placed. (Computed in FINOFF.) 
Row 6: The J-th column in the 6tin row of ISPS contains the length of the J-th set within the RD-vector. That is, it is the total amount of space in the RD-vector allocated to storage of elements in set $J$. BLDOFF has computed information necessary to ensure that enough space has been allocated for each set. (Computed in FINOFF.)

LCDS $(I, J):$ Cummulative Dimension Size of Dimension I is Labelled Common $J$. This table is also created in BLDTBL. The $\mathrm{J}$-th column corresponds to the $\mathrm{J}$-th labelled common, $\mathrm{J}=1, \ldots, \mathrm{NLC}$. The I-th row corresponds to the I-th dimension, $I=1, \ldots$, NDIM. Given a labelled common index $\mathrm{J}$ and a dimension index $\mathrm{I}$, dimension $\mathrm{L}$ is contained within the same labelled common and is below dimension I in the hierarchy if

$$
0<L C O(L, J)<L C O(I, J):
$$

Define $L(I)$ to be the set of all dimensions for which (1) holds. We know that

$$
|\operatorname{LCS}(L, J)|
$$

is the size of the L-th dimension in the $\mathrm{J}$-th labelled common. Thus

$$
\prod_{\xi \in L(I)}|\operatorname{LCS}(\xi, \mathrm{J})| \stackrel{\Delta}{=} \operatorname{LCOS}(\mathrm{I}, \mathrm{J})
$$


is the product of the sizes of all the dimensions in the hierarchy strictly below the I-th dimension in the J-th labelled common. The LCDS matrix contains only those dimensions that actually vary in the $\mathrm{J}$-th rabelled ${ }^{5}$ common -- those upon which the J-th labelled common are conditional are not included.

$\operatorname{ISKJ}(I, U)$ : Storage Conditionality Matrix. The ISKJ matrix is user input and specifies all conditional storage dependencies in the mode1. In the array isKJ $(I, J), I$ varies over the number of dimensions $I=1, \ldots$, NDIM and $J$ varies over the number of sets $J=1, \ldots$, NSETS. The entry $\operatorname{ISKJ}(I, J)$ is zero if dimension $I$ is not contained in set $\mathrm{J}$ and is one of the dimensions in set $\mathrm{J}$ that is higher than or equal to dimension I if dimension $I$ is in set $J$. The $\operatorname{ISKJ}(I, J)^{\text {th }}$ dimension. is the set dimension upon whose value we wish the storage at dimension I to be conditional. A default option exists within the program to set

$$
I S K J(I, J)= \begin{cases}0 & \text { if dimension } I \text { is not in set } J \\ I & \text { if dimension } I \text { is in set } J\end{cases}
$$

4. Building che Orrsets (BLDOFF)

a. Traversing the System 2000 Unioad File

As discussed above, the System 2000 unload file contains every node in the model tree in the order in which those nodes are traversed according to the algorithm illustrated in Figure III.B.7. We will discuss in some detail the format and contents of the System 2000 unload file and then the procedure for reading it. Below the system tables in the System 2000 unload file (FTO02) 
is a value string that is a result of a "pre-order traversal" of the part of the data tree that contains the model data. This value string must be traversed twice, once by BLDOFF and once by BLDD. The routines OCOMP and DCOMP perform this tree traversal. In this section we will describe in some detail how both OCOMP and DCOMP traverse this tree.

We will illustrate the system 2000 unload file conventions using the tree in Figure VI.B.6. (We have suppressed the nodes and have included the information in the associated boxes in their places.) The unload file appears in Figure VI.B.7. There are 9 nodes and two data sets. We will now discuss the meaning of the integers 111,113 , etc. before continuing onward with our discussion.

Each node in the tree is characterized by a particular string of numbers. Under the System 2000 conventions for our applications, the following conventions pertain. The next tree node in the sequence is signalled by a 2 digit number called a repeating group number. In the present implementation we have assigned the repeating group numbers as follows:

40 - 49: System Table Repeating Groups

11 - 39: Hierarchical Tree Repeating Groups

The system table nodes will be ignored for the moment and we will discuss the hierarchical tree nodes. The level in the tree of a hierarchical node with a repeating group number $R G$ is 


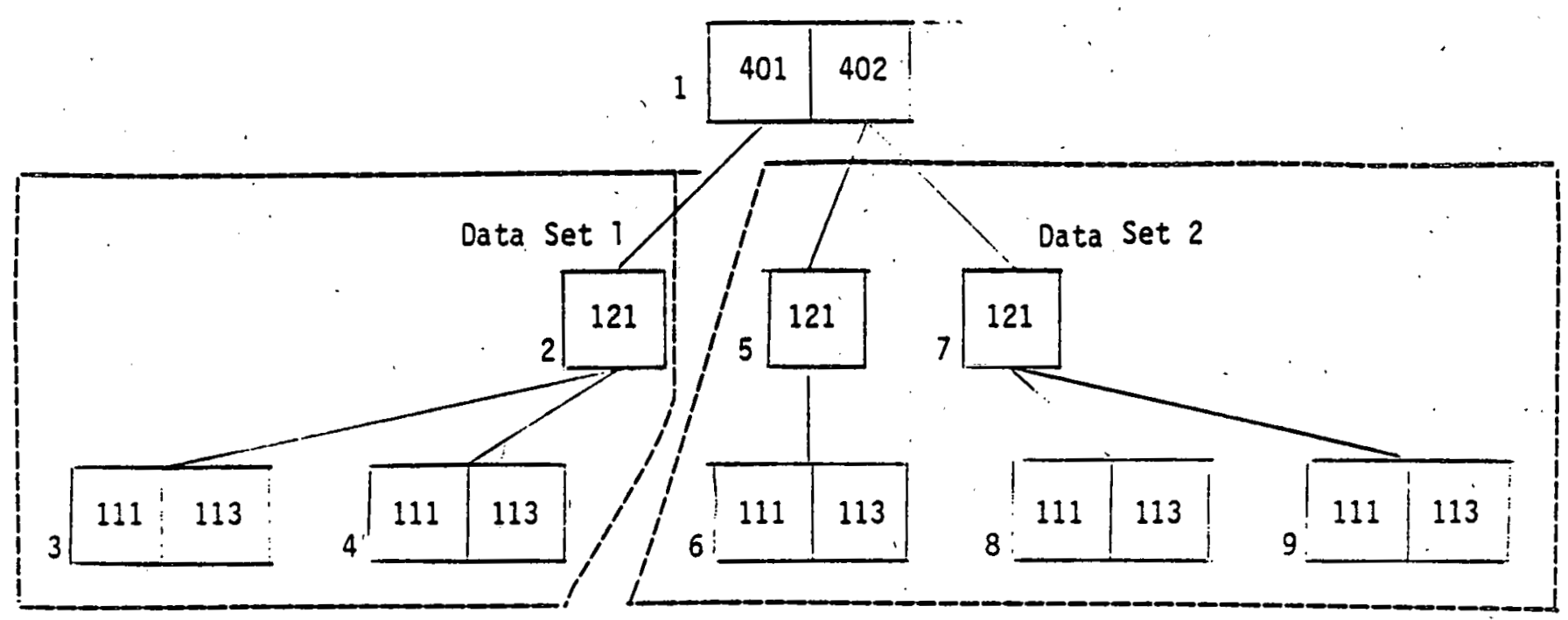

Figure VI.B.6 SAMPLE MODEL INFORMATION TREE 
$401 * 2 \star 402 *$ MODEL2*12*121*0*11*111*1*113*100*11*111*2*113*101*12*121*1*11*111*1* $113 * 200 * 12 \star 121 * 2 \star 11 * 111 * 113 * 210 * 11 * 111 * 2 * 113 * 211 * \mathrm{~F} I N I \star \star$

Figure VI.B.7 SYSTEM 2000 UNLOAD

FILE FOR SAMPLE TREE 


$$
\text { dimension number }=1 \text { evel }=R G-10
$$

Hence, when we encounter a RG number, we can quickly tell at what level in the tree the corresponding node lies.

After the repeating group number will be found a 3 digit number we call an element number. The element number signals that there will be a piece of data associated with the node and tells us what type of data it is. The first two digits must match the repeating group number and the third digit tells us what is the mode of the data attached to the node:

$$
\begin{aligned}
& \text { Mode }=1 \text { implies a name } \\
& \text { Mode }=2 \text { implies a name } \\
& \text { Mode }=3 \text { implies an integer } \\
& \text { Mode }=4 \text { implies a real }
\end{aligned}
$$

Thus, the sequence of characters $* 1+* 114 *$ in the unload file signals a node has just been encountered at level 1 and that a real number is about to be associated with that node.

After the element number comes the piece of data promised. Thus, in the above example, the characters $* 11 * 114 * 3.61 *$ defing a - node at level 1 and attach the number 3.61 to it. If we would like to attach two or more pieces of data, we simply put two or more element numbers, one for each data item as follows.

$$
* 11 * 114 * 3.67 * 112 * \text { PRICE* }
$$

This string defines the node at level 1 and attaches the name 
"PRICE" and the number 3.61 to it. Of course, if we wish to attach no data to the node, we simply put no element number and no data af.ter the repeating group number -- we simply move on to the next repeating group number.

The System 2000 dimensional organization is shown in Table VI.B.1.

b. Establishing the Offsets (BLDOFF/OCOMP)

In this section, we will describe the algorithm used to traverse the hierarchical tree, decompose it into sets, and establish offsets for each dimension ir each set. This procedure is accomplished using a relatively complex piece of logic called subroutine OCOMP which is called from BLDOFF. We will describe. it in some detail as it is central to the present implementation. We will begin our discussion bý describing a small element of logic that has been built to traverse the System 2000 unload file and identify repeating groups, element numbers, names, and data. The flow chart for this logic appears in Figure VI.B.8. The logic is quite simple. Beginning at point (A) in the figure, we begin by reading the next piece of information between the *'s in READST. Based on the mode of the previous string, we decide whether to ignore the new string or process it. If the mode of the previous string was 1 or 2 , we know it was an element number that indicated a name. We thus ignore it, reset mode to 5 , and read the next string. If the mode of the previous string was 


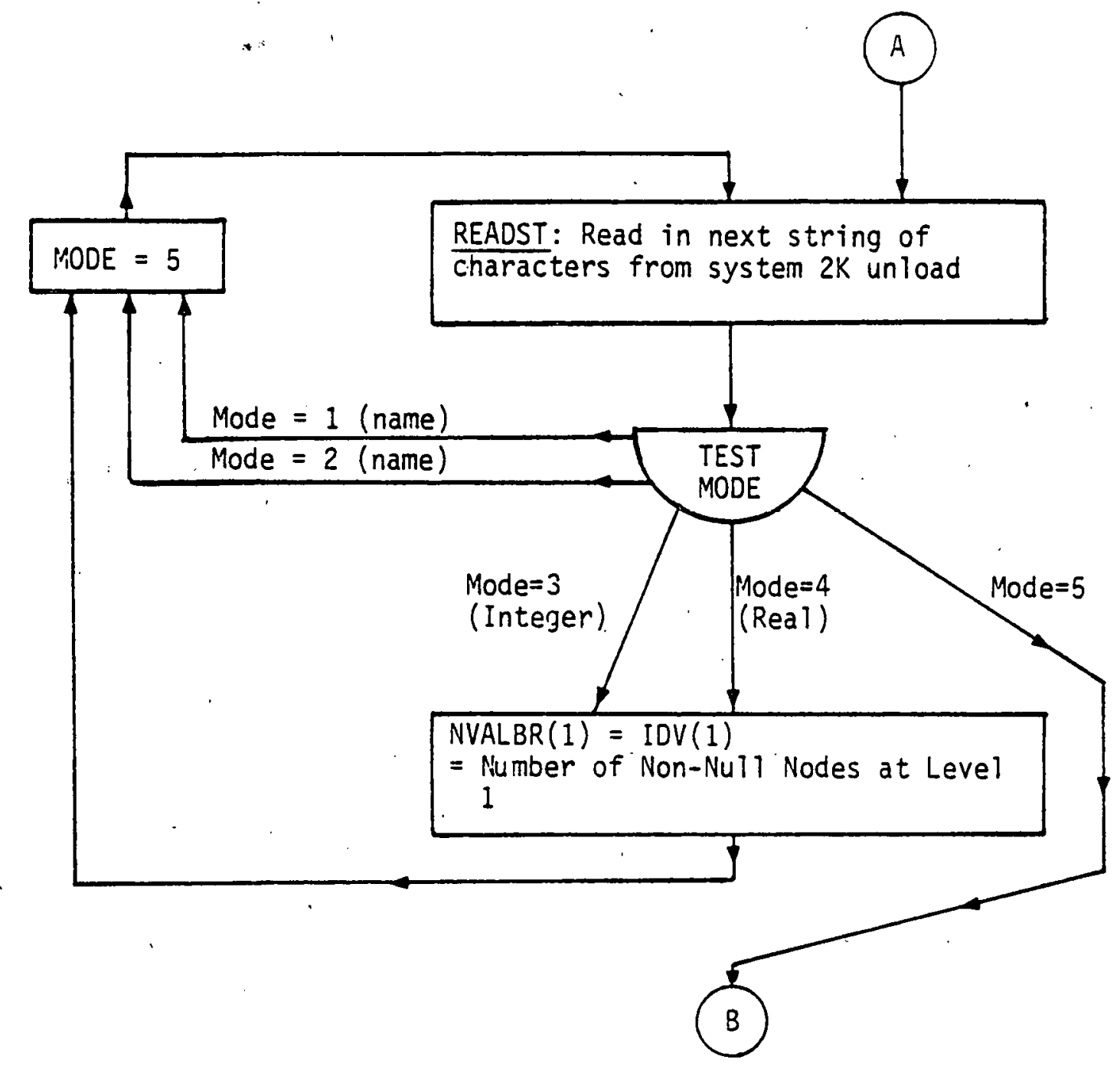

Figure VI.B.8. READING THE SYSTEM 2000 UNLOAD FILE IN BLDOFF 
TABLE VI.B.I

Definition of the data base.

4.10\%: MIIIEL IFISI:

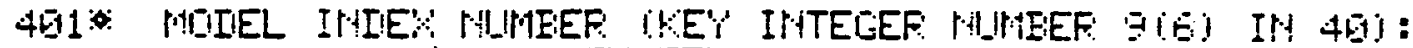

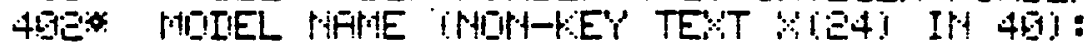

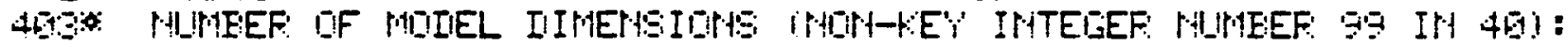

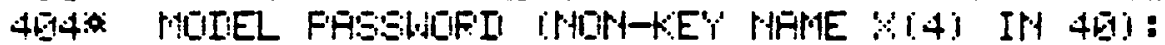

40 IO THELE FIIU IFI IN 401]:

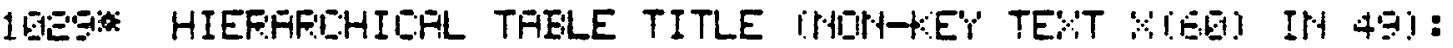

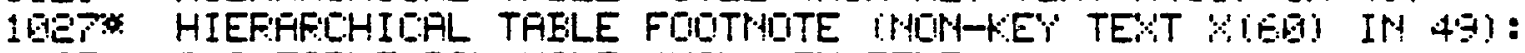

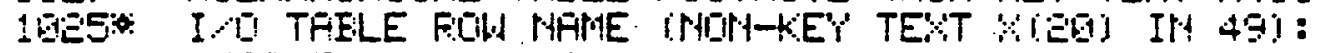

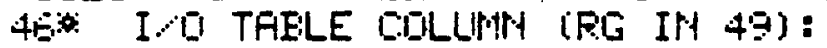

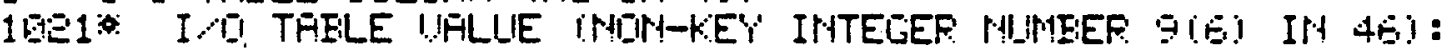

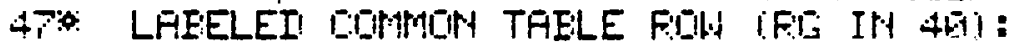

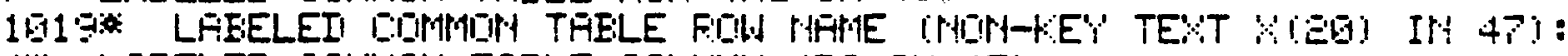

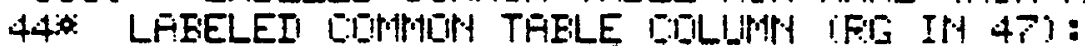

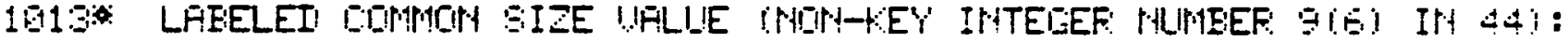

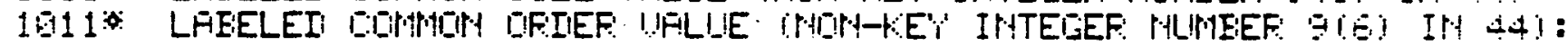

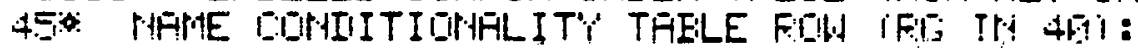

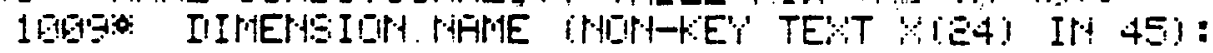

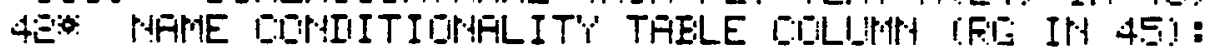

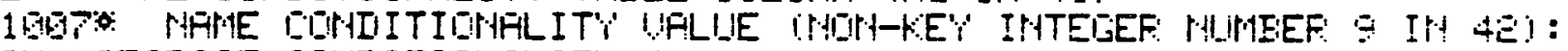

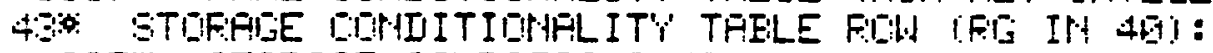

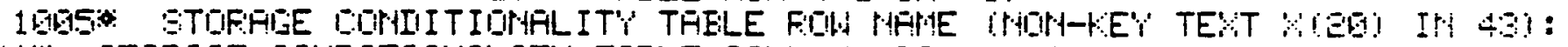

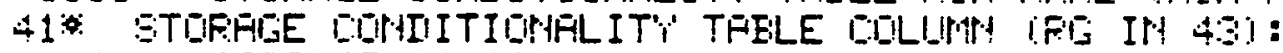

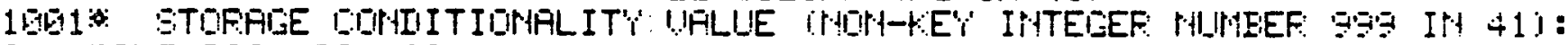

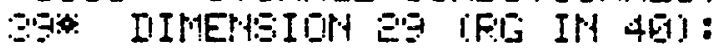

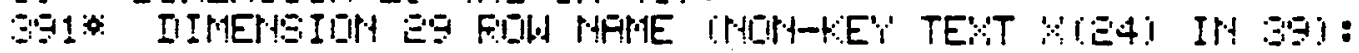

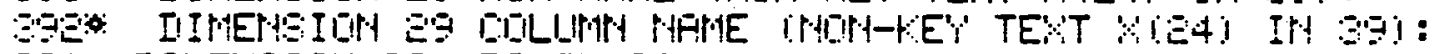

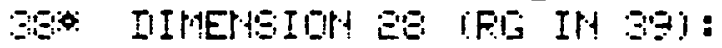

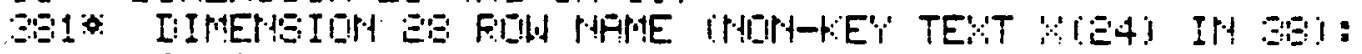

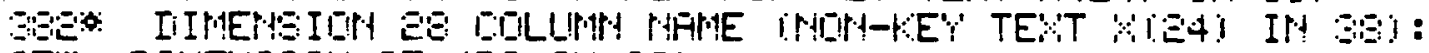

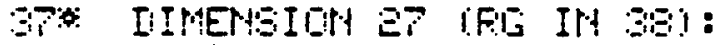

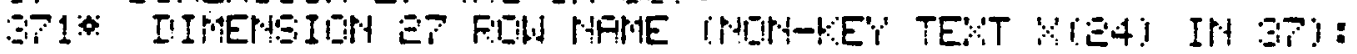

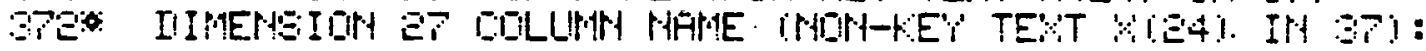

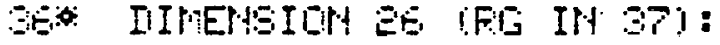

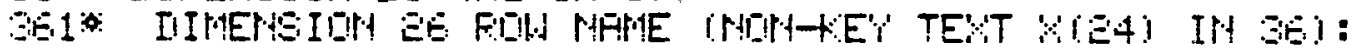

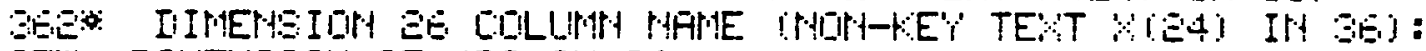

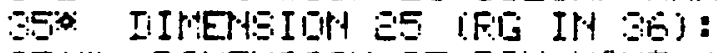

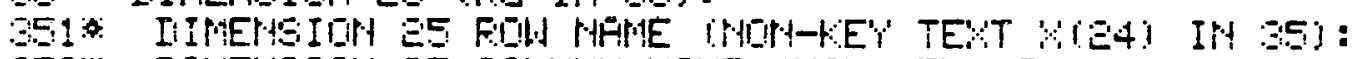

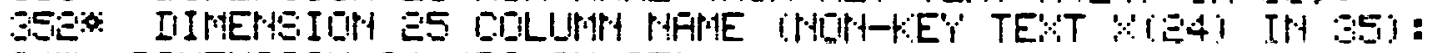

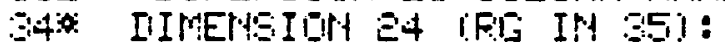

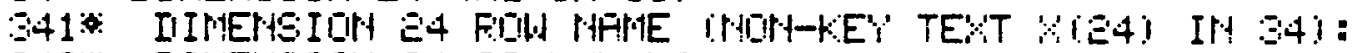

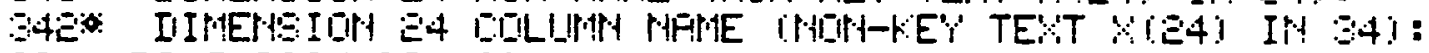

OM IIMEHSIDHA E

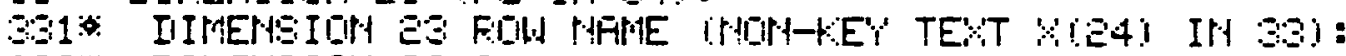

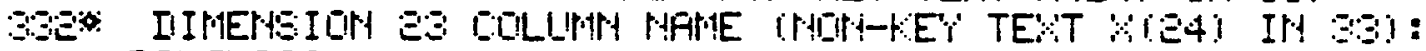

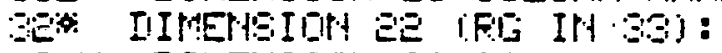

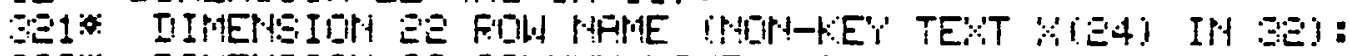

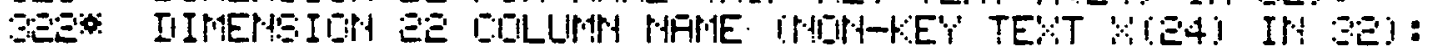

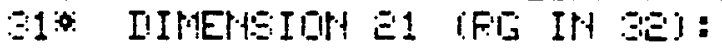

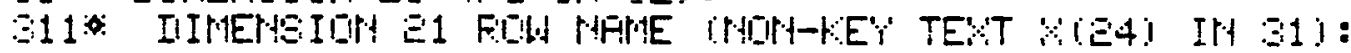

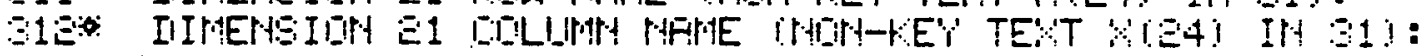

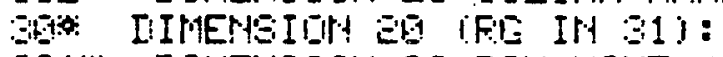

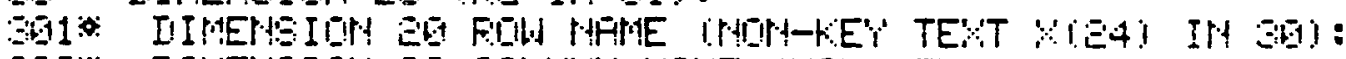

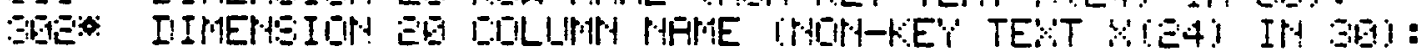


TABLE VI.B. 1 (continued)

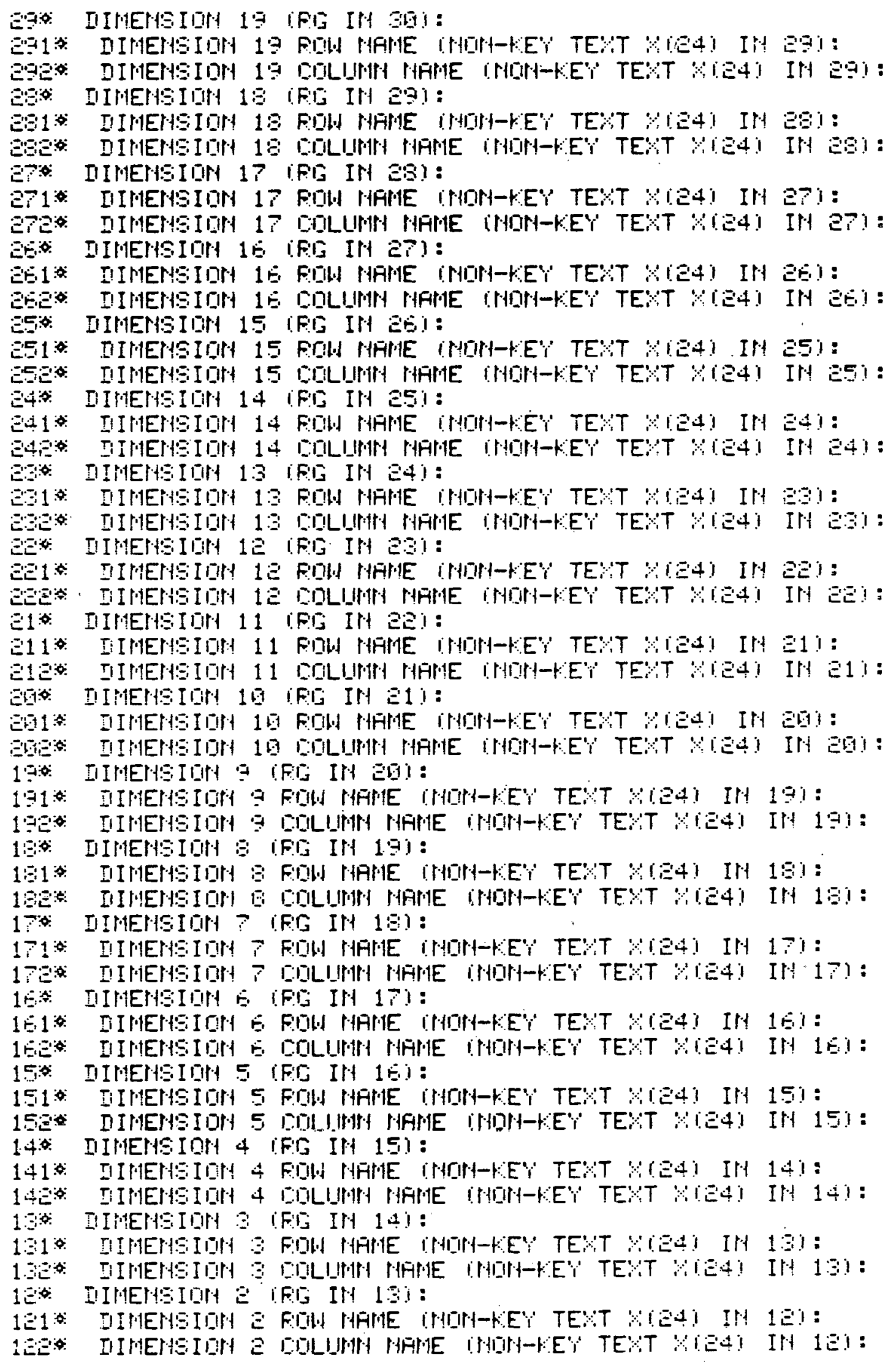


TABLE VI. B. 1 (continued)

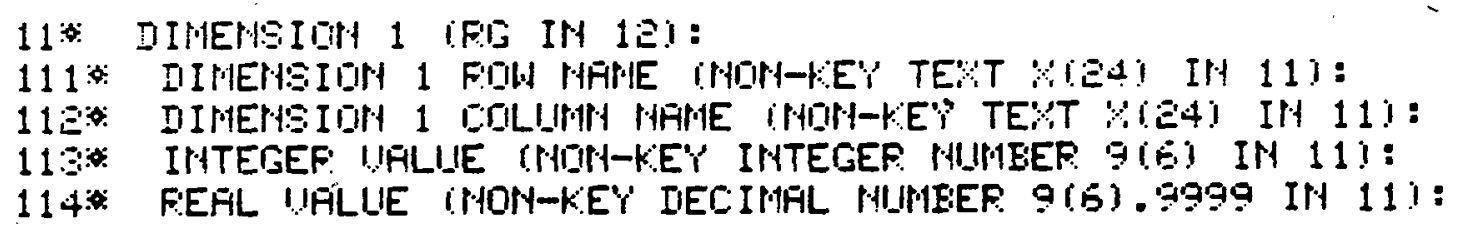


3 or 4 , we know it was an element number that pointed to an integer or real number and thus was at level 1 in the tree. We record the fact that a piece of valid data was recorded at level 11 ,

$$
\operatorname{NVALBR}(1)=\operatorname{IDV}(1)
$$

reset mode to 5 , and read the next string. Finally, if the mode is 5 , we have a repeating group number or an element number to process. We do so by going to (B) and calling the routine OCOMP.

Proceeding onward to Figure V.B.9, we begin at, B by testing whether the new character string is of length 2 (repeating group) or length 3 (element number). In the former case, we reset mode $=5$ and in the latter we set mode to the last digit of the element number. In either case, we set

$$
\begin{aligned}
J H D I M=R G-10= & \text { dimension number of the repeating } \\
& \text { group or element number just } \\
& \text { encountered. }
\end{aligned}
$$

If mode $=1,2,3$, or 4 , we do not have a repeating group number and we go to $(\mathbb{X}$ which will be described below. If mode $=5$ we have a repeating group and thus we know we have just encountered a new node in the tree. In this case, we record IWe will discuss the meaning of this "branch validation" calculation
below. 


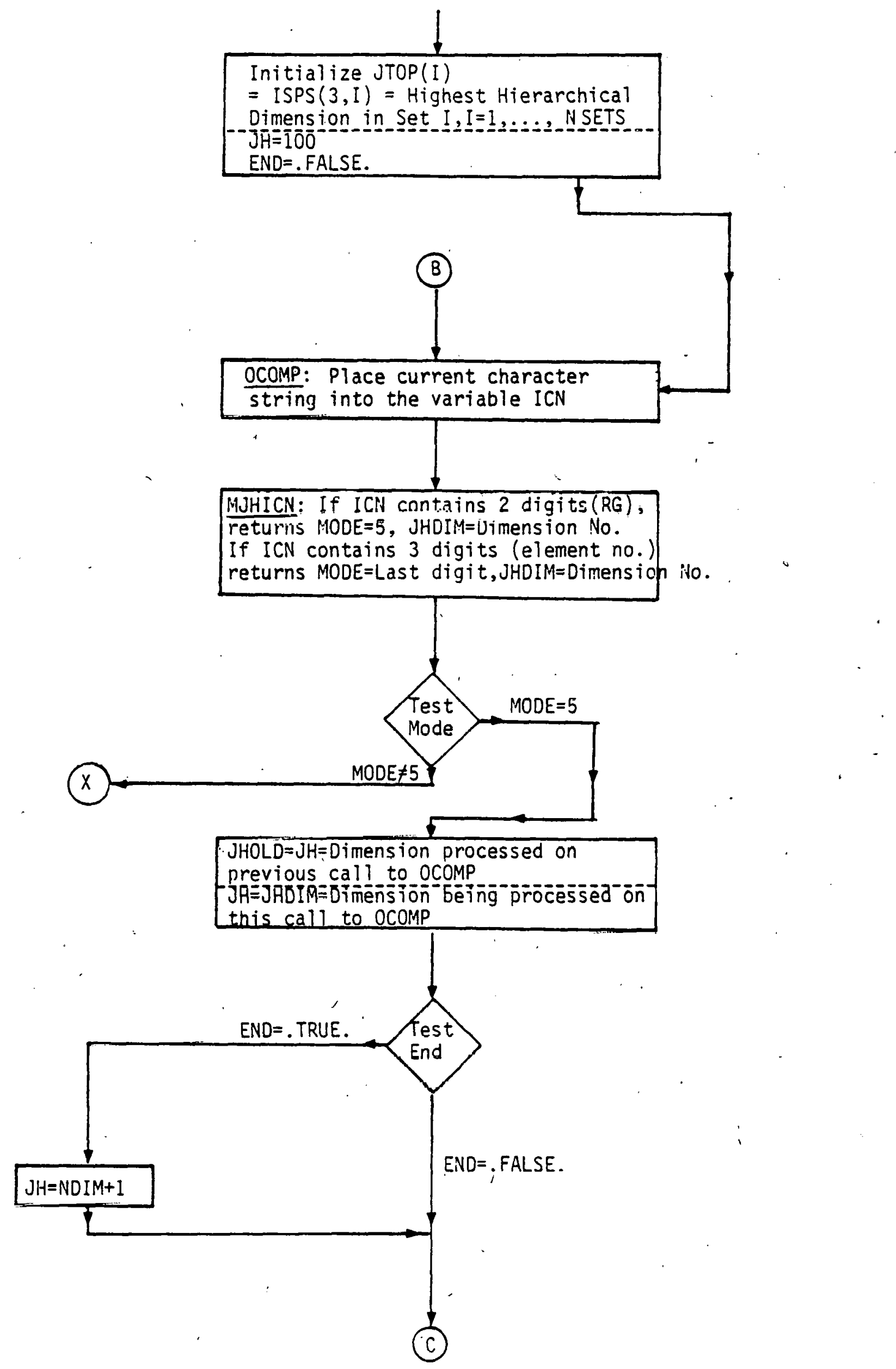

Figure VIB.9: Testing Mode in OCOMP 


$$
\begin{aligned}
& J H O L D=\text { dimension of node we just left } \\
& J H \quad=\text { dimension of node we just entered }
\end{aligned}
$$

and test whether we are finished with the entire system 2000 unload. If so, we set $\mathrm{JH}=\mathrm{NOIM+1}$ so that the entire tree is updated one final time in the manner we will describe below. In either case, we proceed to (C) to process the new node in the tree.

The logic used to process the tree structure appears in Figure VI.B.10. Beginning at (C), we first determine the relationship between the level of the node we just left and the node we just entered.

Case 1: JH < JHOLD - we have moved down the tree

Case 2: JH = JHOLD -- we have moved across the tree at the same dimension.

Case 3: JH > JHOLD -- we have moved up the tree.

These three cases will be explored in detail below.

Case 1: In this case, we have moved down the leftmost branch of the tree one dimension below JHOLD. We thus initialize

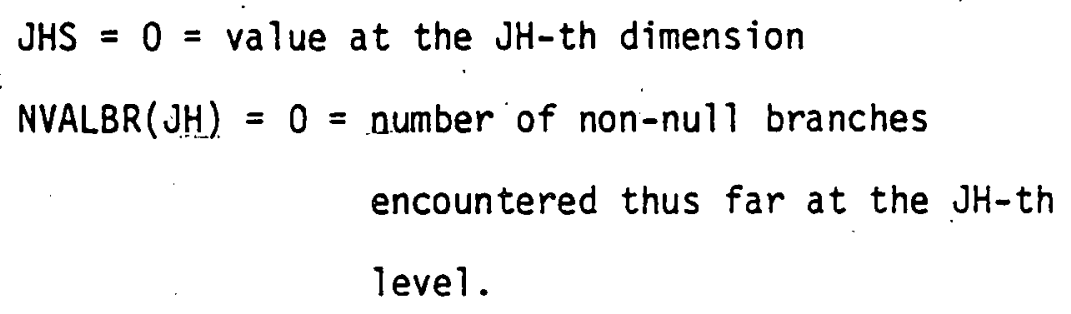




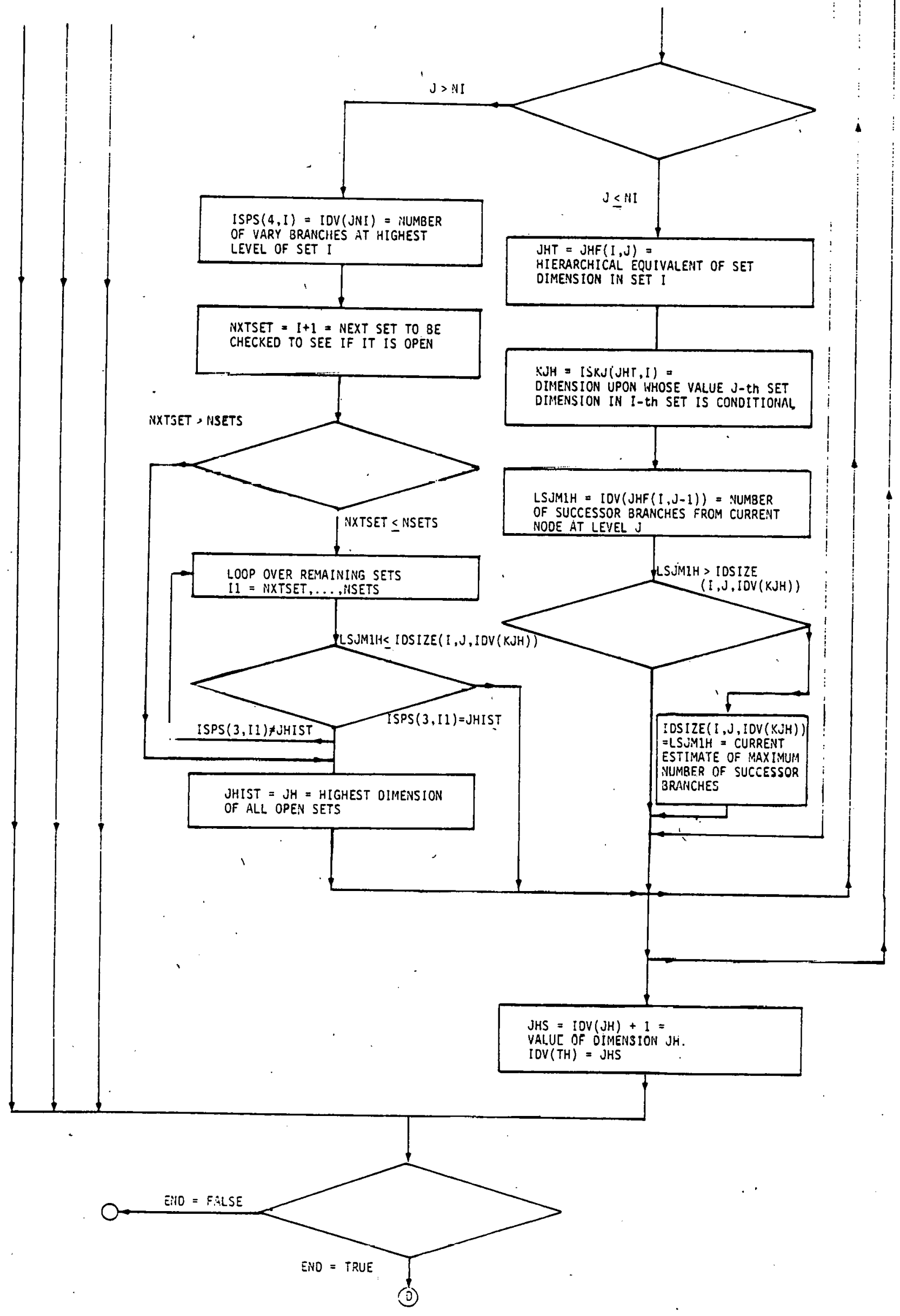

Figure VI3.10: TRAVERSING THE HIERARCHICAL TREE STRUCTURE IN OCOAP 
Figure V1.8.10

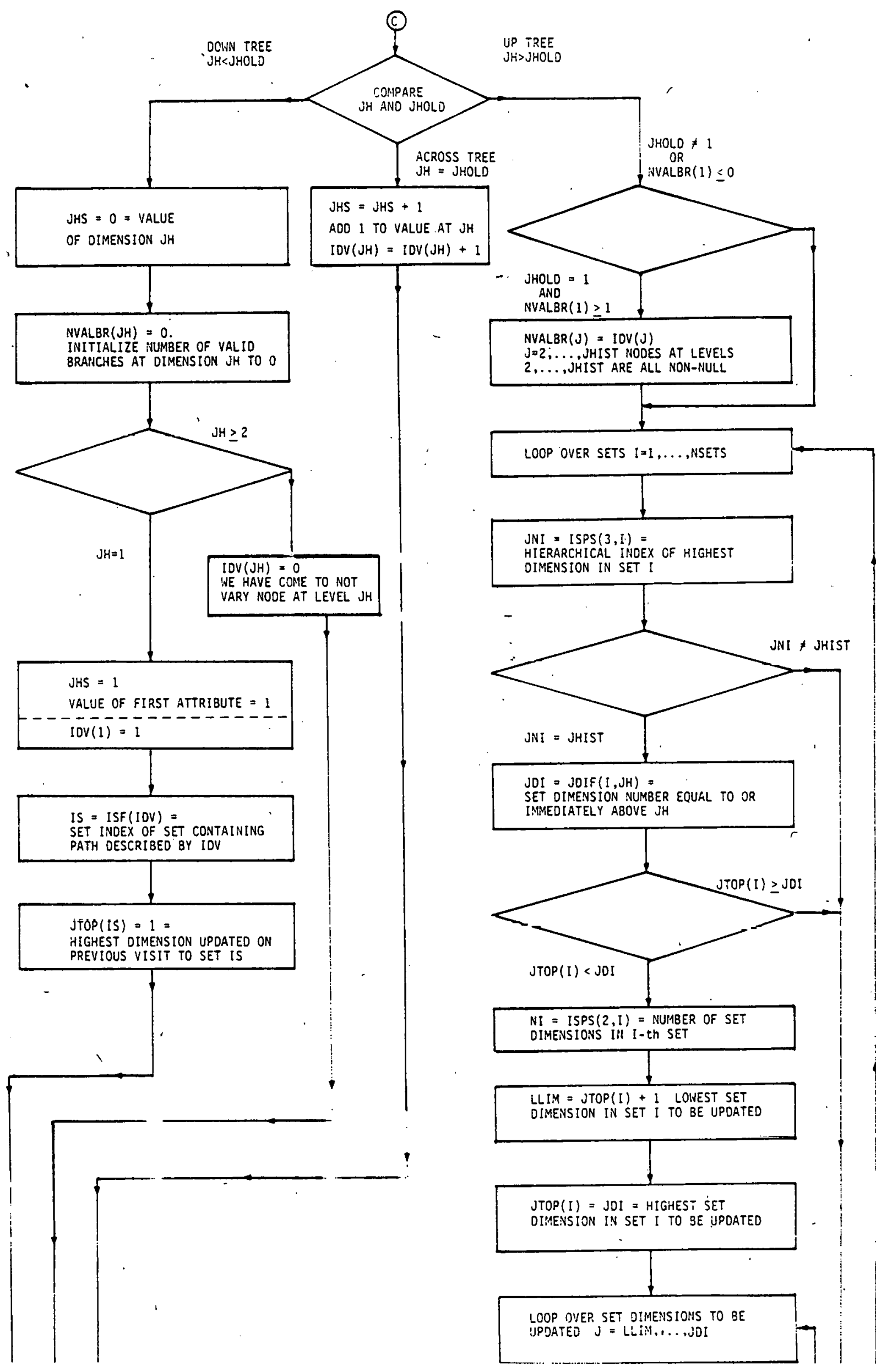


We then test whether we are at the bottom level (attribute), in which, case $\operatorname{IDV}(1)=1$ and JHS $=1$ by convention, or whether we are at level 2 or higher, in which case $\operatorname{IDV}(\mathrm{JH})=0=\mathrm{JHS}$. If we are at level 2 or higher, we have finished with this new node and can proceed to the next node.

If we are now at level 1 , i.e., $J H=1$, we must record the set in which the IDV vector lies. This set is uniquely determined by the non-zero elements (i.e. the dependence branches) in the IDV vector. We also must initialize JTOP(IS) $=1$, recording the fact that we have just entered (or reentered) set IS. The variable JTOP(IS) will keep a running record of the highest set dimension in set IS updated on the previous visit to set IS. The reader will note in Figure VI.8.9 that JTOP(I) was initialized to the number of set dimensions in set I before OCOMP was ever entered. As we shall see shortly, this initialization ensures that the Is th set will not be updated before it is initially entered and JTOP(IS) reset to 1 . Upon completion of these tasks, we have completed processing dimension $\mathrm{JH}$ and we proceed to $\mathrm{X}$ to get a new node in the tree.

Case 2: In this case, we have moved across the tree to the next sibling at a particular level. If $\mathrm{JHOLD}=\mathrm{JH} \geq 2$, we know that the old node had no data and was either a "placeholder" or a null node. If on a subsequent node visit we jump down before jumping up, it was a placeholder. If we jump up before jumping down, it was a null node. In this "across" case, we need only add 1 to the 
value of JHS at level JH and, 1 to the current value of IDV(JH) to record our new position in the tree. When this is complete, we go to $X$ and get a new node in the tree.

Case 3: In this case, we must evaluate all the information that was gathered in cases' 1 and 2 since the last jump up. It is here where we establish the conditional widths of the set trees as discussed in Chapter III.

The first step is to determine whether any null nodes have been identified since the last upward jump. In order to do so, we instead determine how many non-nu17. nodes have been identified. We can do so by noting the following general property of the tree. If a path IDV goes completely through the tree to level 1 and there is a piece of numerical data at. level 1 , then branches

$$
\begin{aligned}
& 1,2, \ldots, \operatorname{IDV}(\text { NDIM) are non-null at level NDIM } \\
& 1,2, \ldots, \text { IDV (NDIM-1) are non-null at level NDIM-1 } \\
& i, 2 \ldots, \text { IDV(1) are non-null at level } 1 .
\end{aligned}
$$

Hence, we begin by testing whether JHOLD $=1$ and NVALBR $(1) \geq 1$. That is, have we jumped up from level $I$ and did we find at least 1 valid branch with data attäched at level 1 . If so, we know that there are no null branches at levels $2, \ldots$, JHIST, .., NDIM. That is,

$$
\operatorname{NVALBR}(J)=\operatorname{IDV}(J), J=2, \ldots, J H I S T
$$


The number of null branches at level 1 of course will be $\operatorname{IDV}(1)-\operatorname{NVALBR}(1)$.

If JHOLD $\neq 1$ or $\operatorname{NVALBR}(1) \leq 0$, that is if we have jumped up from level 2 or above, we know we have null branches at level 2 and perhaps above. The number of null branches at each level is

$$
\operatorname{IDV}(J)-\operatorname{NVALBR}(\mathrm{J}), \mathrm{J}=2, \ldots, \mathrm{JHIST}
$$

which we print out in the code. ${ }^{1}$

Having identified the null nodes, we then proceed to update our estimates of the maximum conditional sizes required at, cach level for each set. Define

$$
\begin{aligned}
\operatorname{IDSIZE}(I S, J, K J S)= & \text { maximum number of immediate } \\
& \text { successor branches from nodes } \\
& \text { at set dimension } \mathrm{J}^{2} \text { in set. IS } \\
& \text { conditional on the value KJS } \\
& \text { of the user specified } \\
& \text { conditional dimension. }
\end{aligned}
$$

We w1Il update IDSIZE to account for the nodes we have visited since the last jump up to level JH. We begin by looping over sets $I=1, \ldots$, NSETS. We first calculate

$$
\begin{aligned}
J N I=\operatorname{ISPS}(3, \mathrm{I})= & \text { hierarchical index of the } \\
& \text { highest level dimension in set } I .
\end{aligned}
$$

We do not automatically eliminate the null nodes. We merely identify then so that the user can eliminate them manually using the Executive.

${ }^{2}$ Set dimension number refers to the indexing of dimensions defined only other those dimensions which are dependent in that set. Hierarchical dimension number refers to the indexing over all model dimensions. 
We then compare this dimension with JHIST, which is the hierarchical dimension at which all se's presently under consideration begin. We will discuss how JHIST is arrived at below. If JNIfJHIST, we know that set I is not "open", i.e., either we have previously jumped above its uppermost dependence branch and "closed" it or we have never jumped as high as JHIST before and thus have not yet "opened" it.

If, however, JNI=JHIST, we know that set I is open and some of the estimates of IDSIZE must be updated to account for the nodes that have been visited since the last jump up to dimension JHIST. If JNI=JHIST, we compute the set dimension JDI that is equal to or immediately above hierarchical dimension JH, i.e.

$$
J D I=J D I F(I, J H)^{I}
$$

We next test whether $\operatorname{JTOP}(I) \geq J D I$, that is whether the last update made to this set included a dimension at or above JOI. If so, we know that we have not returned to the 1 level for this set since that update and hence have no new information with which to pérform a further update. If, however, JT.OP(I)<JOI, we know that the dimensions JTOP(I)+1,., JDI can now be updated. In order to do so, we set

$$
\begin{aligned}
& N I=I S P S(2, I)=\text { number of dimensions in the Ith set } \\
& \text { LLIM=JTOP }(I)+1=\text { first dimension to be updated }
\end{aligned}
$$

and we set

$$
\operatorname{JTOP}(I)=J D I
$$

1JOIF is a utility function defined at the end of this chapter. 
in order to tell future steps in the traversal that we have updated up to set dimension JOI since our last visit to a numerical value at level $I$ in set I.

We next set up a loop over set dimensions $J=L L I M, \ldots$, JDI for whose values we wish to update our estimates of IDSIZE. The first step is to test whether $J>N I$, in which case we know that we have just incremented $J$ one set dimension past the top. In this case, we save

$$
\begin{aligned}
\operatorname{ISPS}(4, I)=\operatorname{IDV}(J N I)= & \text { number of dependence branches } \\
& \text { at the highest level in set I. }
\end{aligned}
$$

We then must test whether there are any other sets which are open, i.e., whose highest dimension is JHIST. If so, we go on to the next set $I+I$ and repeat this procedure. If not, we must update JHIST to be equal to $\mathrm{JH}$, indicating that we have closed all sets beginning at the old level JHIST and wish to open all sets beginning at the new level JH. After incrementing JHIST in this fashion, we get the next higher value of $I$ and repeat the calculation, thus opening the sets beginning at JHIST.

In the event that $\mathrm{J} \leq N I$, we know that we are still considering a set dimension $J$ that lies in the Ith set. The series of logic we are about to describe is the basic logic for updating IDSIZE for set I. We begin by finding 


$$
\begin{aligned}
& \mathrm{JHT}=\operatorname{JHF}(\mathrm{I}, \mathrm{J})=\text { hierarchical equivalent of set } \\
& \text { dimension } \mathrm{J} I
\end{aligned}
$$

and finally

$$
\begin{aligned}
\operatorname{LSJMIH}=\operatorname{IDV}(J H F(I, J-1))= & \text { value of } J-1 \text { st set dimension } \\
& \text { conditional on all higher } \\
& \text { ievel values of IDV. }
\end{aligned}
$$

We know that LSJMIH is the number of immediate successor branches at level $J$ conditional on all higher values of IDV, i.e., $\operatorname{IDV}(\mathrm{JH}), \ldots, \operatorname{IDV}(\mathrm{NDIM})$. Under the storage conditionality assumption we will remove all but one of these higher levels of conditionality by maximizing. Our current estimate of the maximum width at set dimension $\mathrm{J}$ conditional on the value of dimension $\mathrm{KJH}$ is

$$
\operatorname{MAX}\{\operatorname{LSJMIH}, \operatorname{IDSIZE}(I, J, \operatorname{IDV}(K J H))\}=\operatorname{IDSIZE}(I, J, \operatorname{IDV}(K J H))
$$

The reader will note that wo have retained the conditional dependence of the width at level $\mathrm{J}$ on the value IDV(KJH) -it appears as an argument of IDSIZE. However, we have retained none of the other conditionality. Having completed this update in our estimate of IDSIZE, we move up to the next set dimension $\mathrm{J}$ to be updated.

$1_{\text {JHF }}$ is a utility function defined at the end of this chapter. 
When we have completed the above steps, we will have updated all estimates of IDSIZE that can be updated given the jump we have just recorded from JHOLD up to JH. We thus record our new position IDV $(J H)$ at the new level, find the new value of the JHth dimension, and proceed to $X$ to obtain a new node.

c. Updating the Offsets (BLDOFF) 1

After traversing the entire data tree, we will have determined. $\operatorname{IDSIZE}(I, J, K J S)$ for all sets $I=1, \ldots$, NSETS, all dimensions $J$ in each set $I$, and all values KJS of the dimension upon whose value the $J$ th set dimension in set $I$ is conditional. Hence, we will know the size of all conditional fans in terms of immediate successor branches in the hierarchical tree as shown in Chapter III. Using this information, we now wish to determine the number of ultimate successor branches at each node in the tree. In order to do so, we use the logic illustrated in Figure VI.B.11. We begin by looping over all sets $I=1,2, \ldots$, NSETS. The next step is to determine the number of dimensions in set I:

$$
N I=\operatorname{ISPS}(2, I)
$$

and to test whether $N I=1$ or $N I \geq 2$. If there is only one dimension in the Ith set $(N I=1)$, we know that the number of ultimate successors at level 2 is

$$
\operatorname{IOFF}(I, 2, K J S)=\operatorname{IDSITEE}(I, 2, K, J S)
$$

This procedure was illustrated in some detail in chapter III. The reader should thoroughly familiarize himself with that material before attacking this section in detail. 


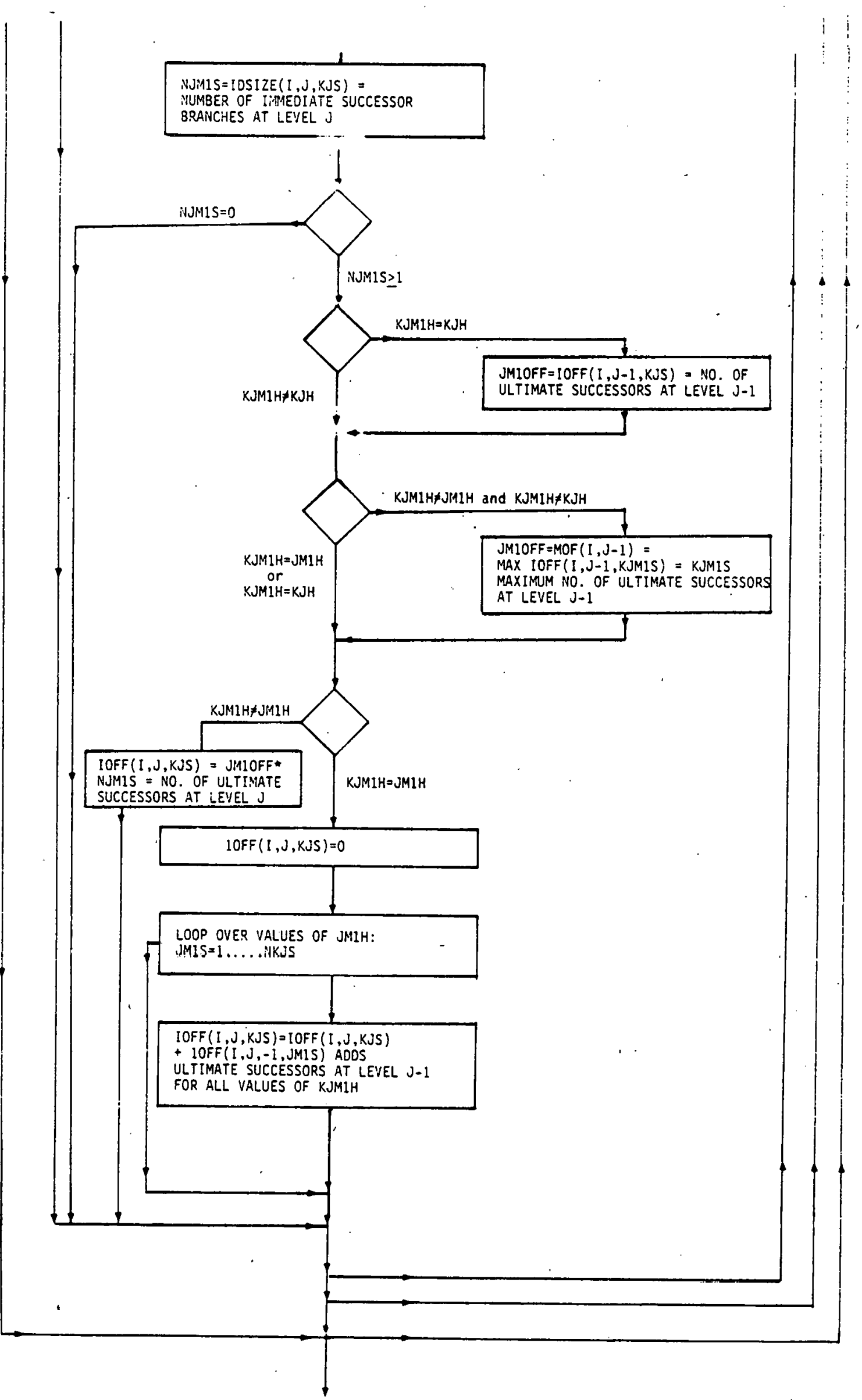

Figure VI.3.11: CALCULATION OF OFFSETS 
Figure VI.B.11

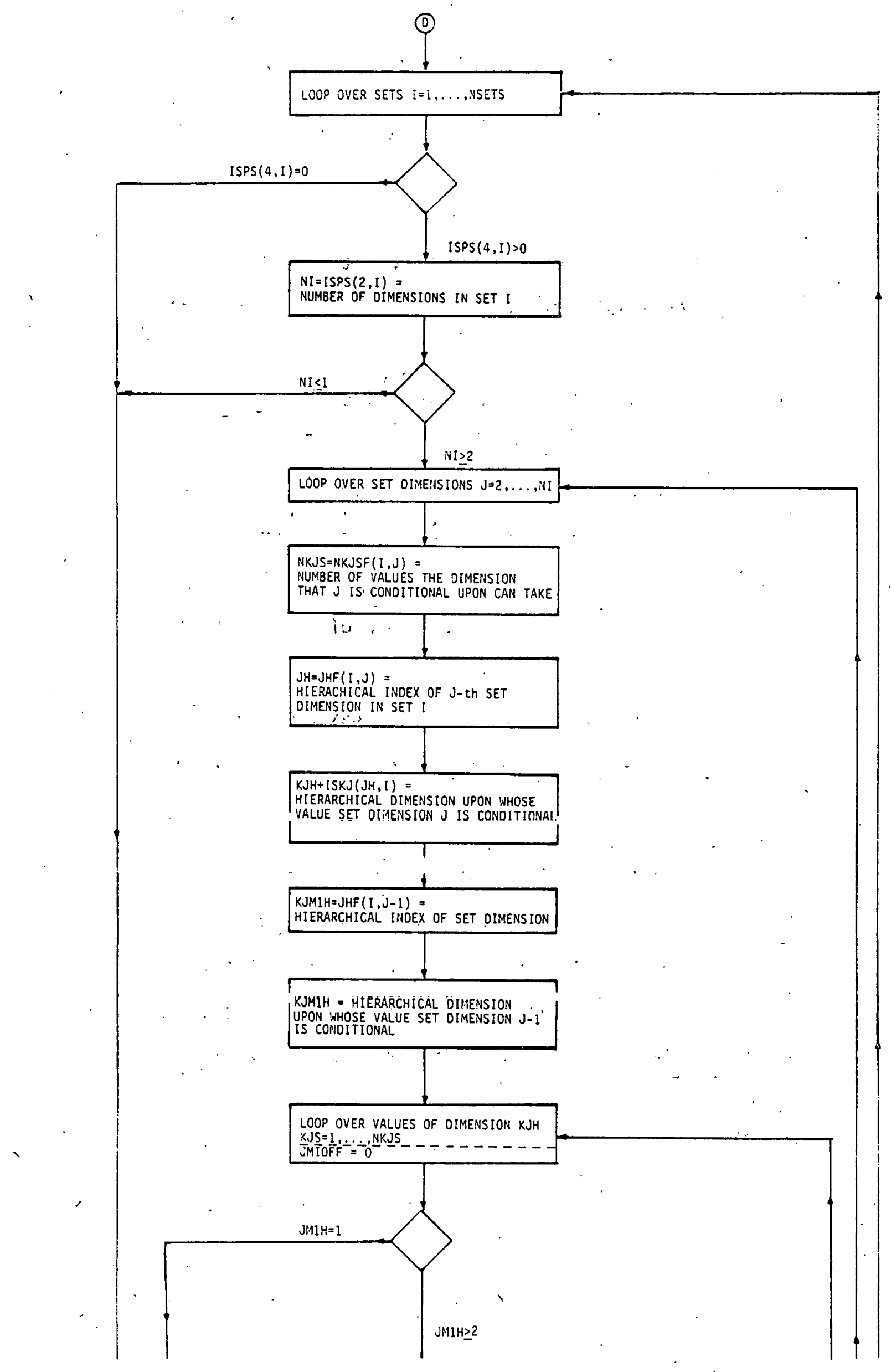


and no calculation is necessary.

In the event that $N I \geq 2$, we must loop over the 2 or more set dimensions $\mathrm{J}=2, \ldots, N \mathrm{~N}$ and calculate:

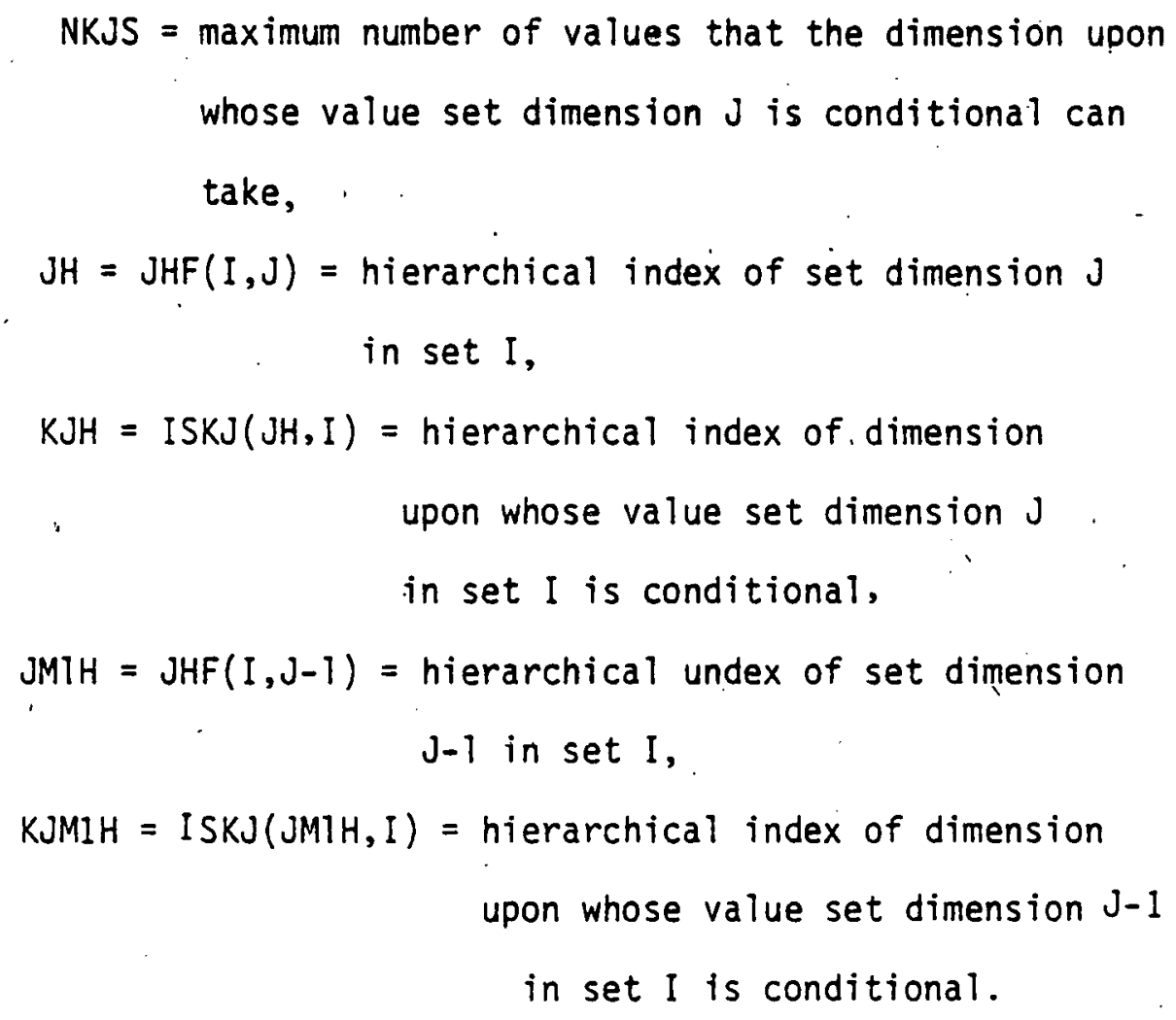

Given these parameters, we then loop over the values $K J S=1, \ldots$, NKJS of the dimension KJH upon whose value $\operatorname{IOFF}(I, J, K J S)$ will be conditional. In each case we will use IOFF at the J-ist level and IDSIZE at the Jth level to recursively determine IOFF at the Jth level. We will consider three cases and will draw an illustrative example for each case: 
Case 1: Dimensions $\mathrm{J}-1$ and $\mathrm{J}$ are both conditional on the same higher level dimension $\mathrm{KJH}=\mathrm{KJMIH}$. This of course implies that $J-1$ cannot be self conditional, i.e., KJMIHFJMiH.

Case 2: Dimension J-1 is self conditional, i.e., KJMTH=JMTH.

Case 3: The complement of 1 and $2--3-1$ is not conditional on itself nor is it conditional on the same dimension that $\mathrm{J}$ is conditional upon, i.e., KJH $\neq K J M T H$ and $K J M T H \neq J M T H$.

Case 1 can be illustrated graphically as shown in Figure VI.B.12. We know that for each value KJS of dimension $\mathrm{KJH}$, there are

$$
\begin{aligned}
& \operatorname{IDSIZE}(I, J, K J S) \text { immediate successors at level JH } \\
& \operatorname{IOFF}(I, J-1, K J S) \text { ultimate successors at level JMIH. }
\end{aligned}
$$

Hence, we know that the number of ultimate successors at level $\mathrm{J}$ is equal at all $\mathrm{J}$-level nodes and is the product of the two:

$$
\operatorname{IOFF}(I, J, K J S)=\operatorname{IDSIZE}(I, J, K J S) * I \operatorname{OFF}(I, J-1, K J S)
$$

In Case 2, the situation is equally simple. Figure VI.B. 13 illustrates. It is easy to see that the ultimate number of successors at the Jth level given the value KJS is

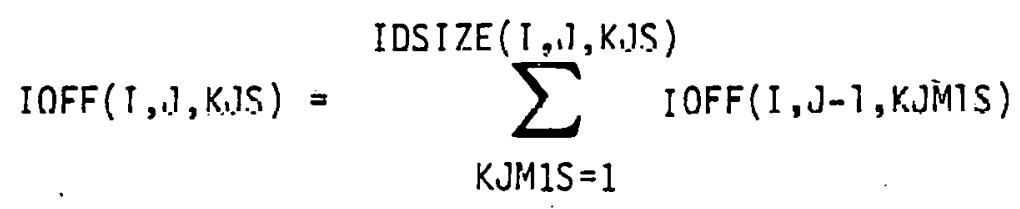




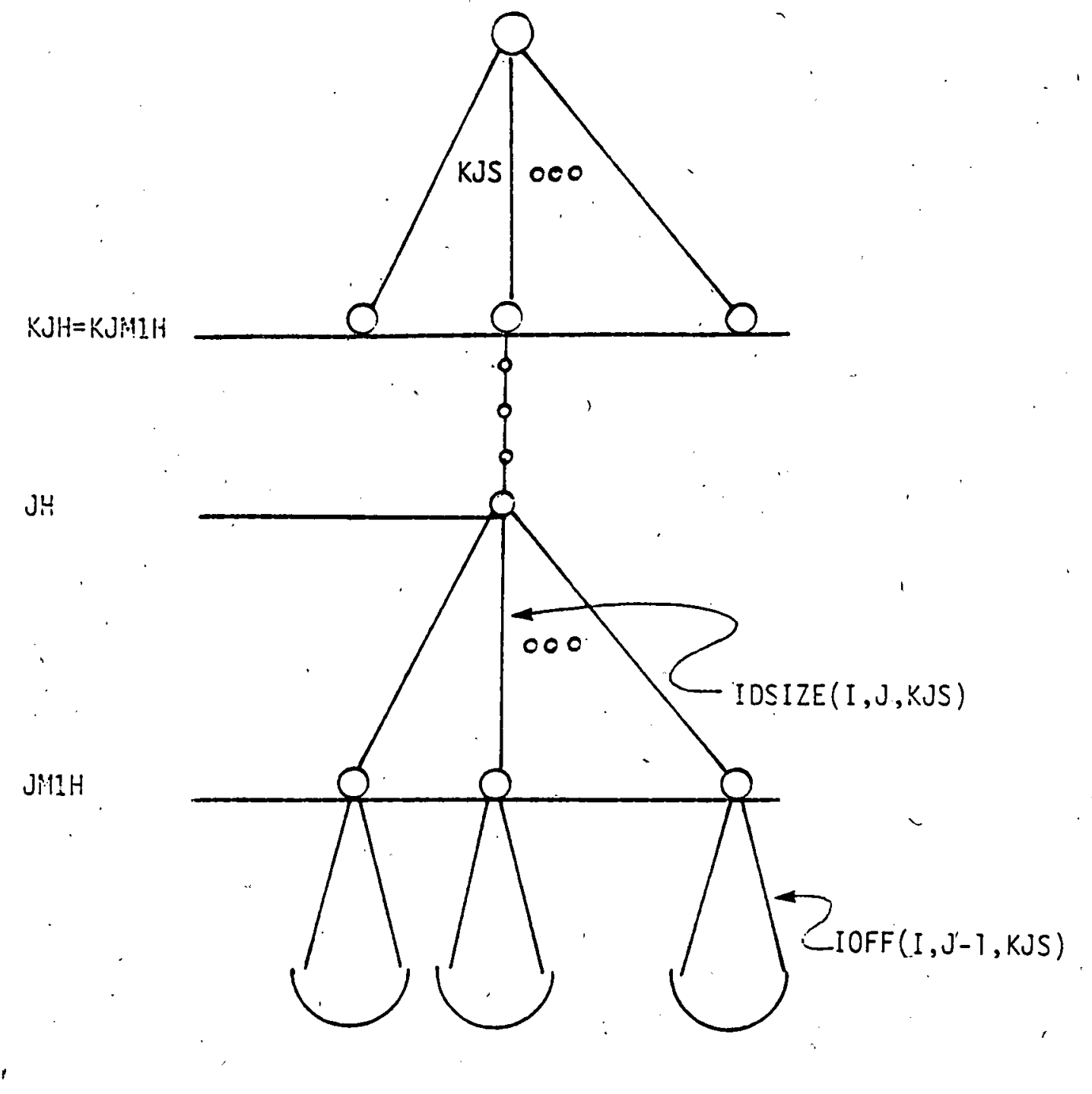

Figure VI.B.12 J-I AND J CONDITIONAL ON SAME HIGHER LEVEL DIMENSION 


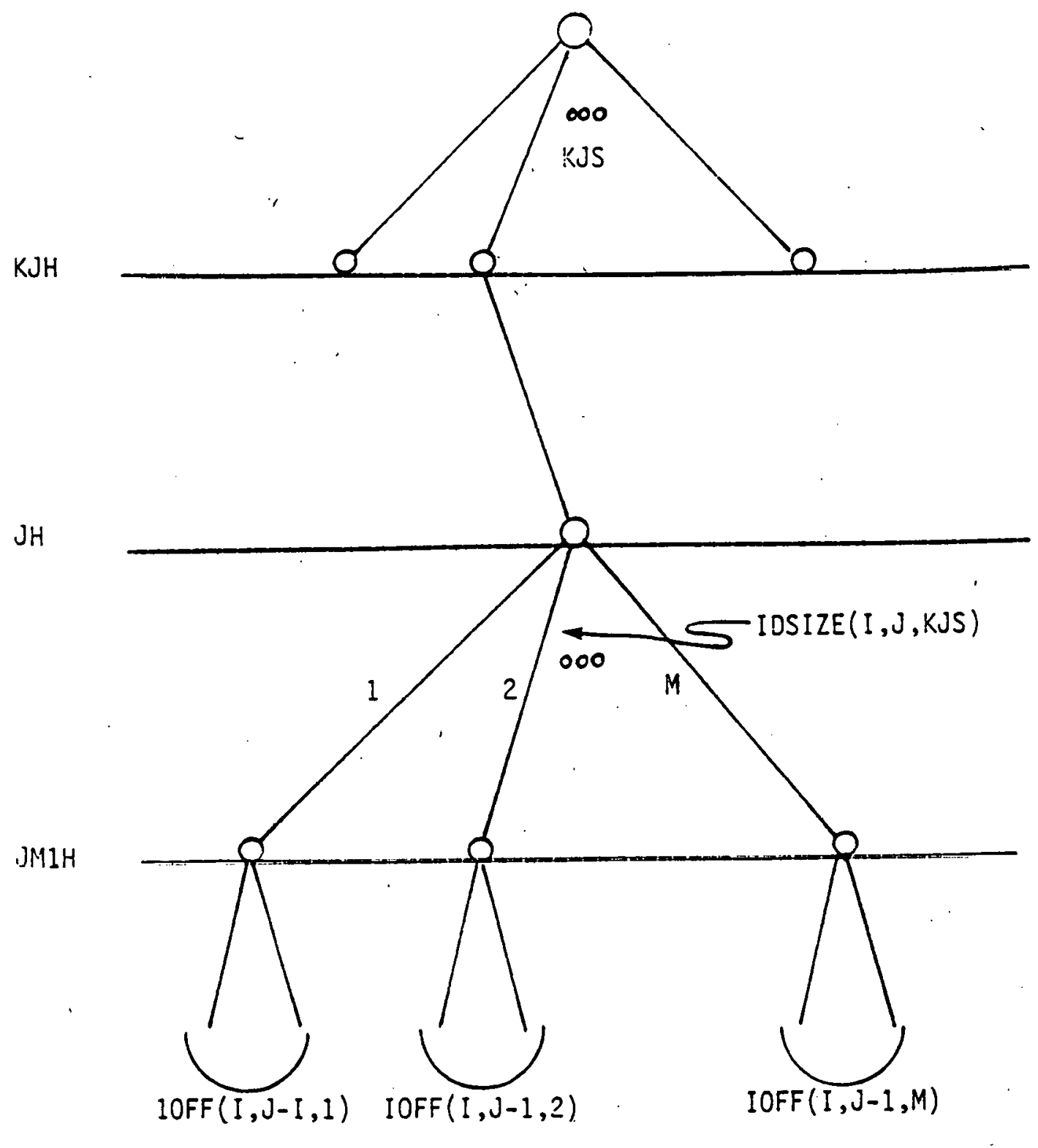

Figure VI.B.13 J-1 IS SELF-CONDITIONAL . 
which is simply the sum of al1 uitimate successors from $J-1$ for each value of $\mathrm{J}-1$.

In Case 3, the situation becomes more difficult. The reason is that level $\mathrm{J}-\mathrm{l}$ is conditional on a dimension at "level $\mathrm{J}$ or higher and level $\mathrm{J}$ is conditional on a different dimension at level $\mathrm{J}$ or higher. This implies two levels of conditionality at level $\mathrm{J}$ rather than the one level we are allowed to have in our offset matrix IOFF. Figure VI.B.14 illustrates this difficulty graphically. In order to resolve this difficulty, suppose we find the largest offset at level J-l over all settings of KJMIS. That is, suppose we find

$$
\begin{aligned}
& \text { MAX IOFF(I., J-1, KJMIS) } \\
& \text { KJMIS }
\end{aligned}
$$

and assume that the offset at level $\mathrm{J}$. is the product of IDSIZE times this maximum:

$$
\operatorname{IOFF}(I, J, K J S)=\operatorname{IDSIZE}(I, J, K J S) *{ }_{K J M I S}^{\operatorname{MAX}} \operatorname{IOFF}(K, J-1, K J M T S)
$$

This will allow sufficient space at the Jth level to accomodate the largest number of successor branches that could possibly occur at the J-1st. level without altering storage at the J-lst level itself.

After completing these calculations for every set I, every dimension J, and: every conditional dimension value KJS, we have the necessary offsets. 


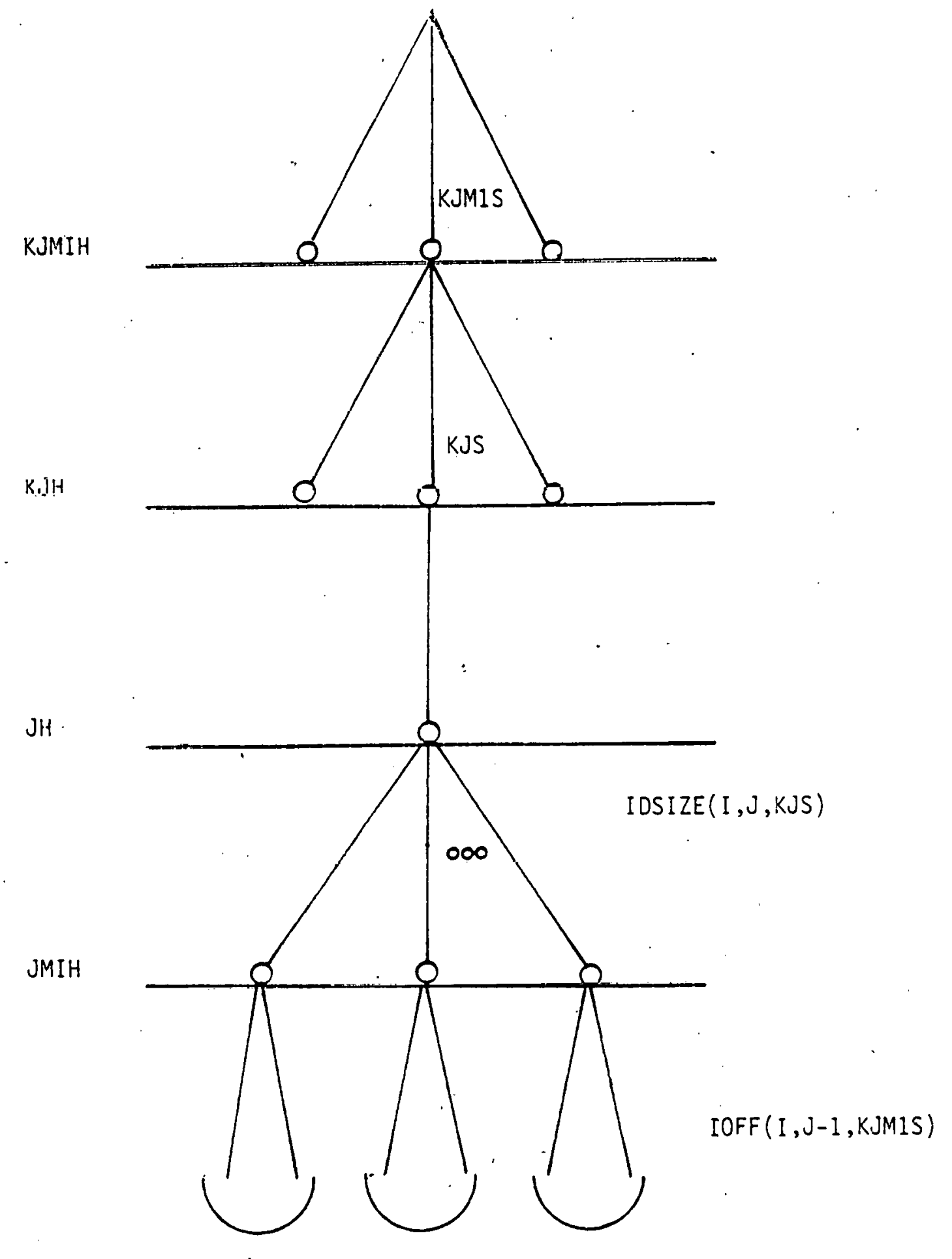

Figure VI.B.14 OUAL CONDITIONALITY 


\section{d. Additional Offset Information (FINOFF)}

Upon completion of the above calculations, we will precompute some of the key parameters that pertain to the RD vector. A flow chart of this calculation appears in Figure VI.B.15. Since the calculations are straightforward, we will not explain the algorithm but, instead, simply summarize the final results:

$$
\begin{aligned}
& \operatorname{ISPS}(5, I)=\text { pointer to the position in the } R D \text { vector } \\
& \text { where the first value of set I will be } \\
& \text { stored, } \\
& \operatorname{ISPS}(6, I)=\text { size of set } I \text { in the RD vector, } \\
& I R D(I)=\text { sum of the lengths of sets } 1,2 \ldots, I-1 \\
& \text { in the RD vector. (This is an initial- } \\
& \text { ization calculation -- these quantities } \\
& \text { will be changed later.) } \\
& \text { LENRD = length of the RD vector. }
\end{aligned}
$$

At this point, it is useful to visualize the structure of the RD vector. Chapter III illustrated the vector in some detail but we shall repeat it here with the above parameters indicated.

Figure VI.B.16 illustrates.

5. Placing Values in the RD Vector (BLDD/DCOMP)

In order to place values into the RD vector in the proper positions, we can traverse the tree in precisely the same order that that BLDOFF/OCOMP did. In the BLDD algorithm we re-traverse the System 2000 unload file, this time picking off the integer and real 


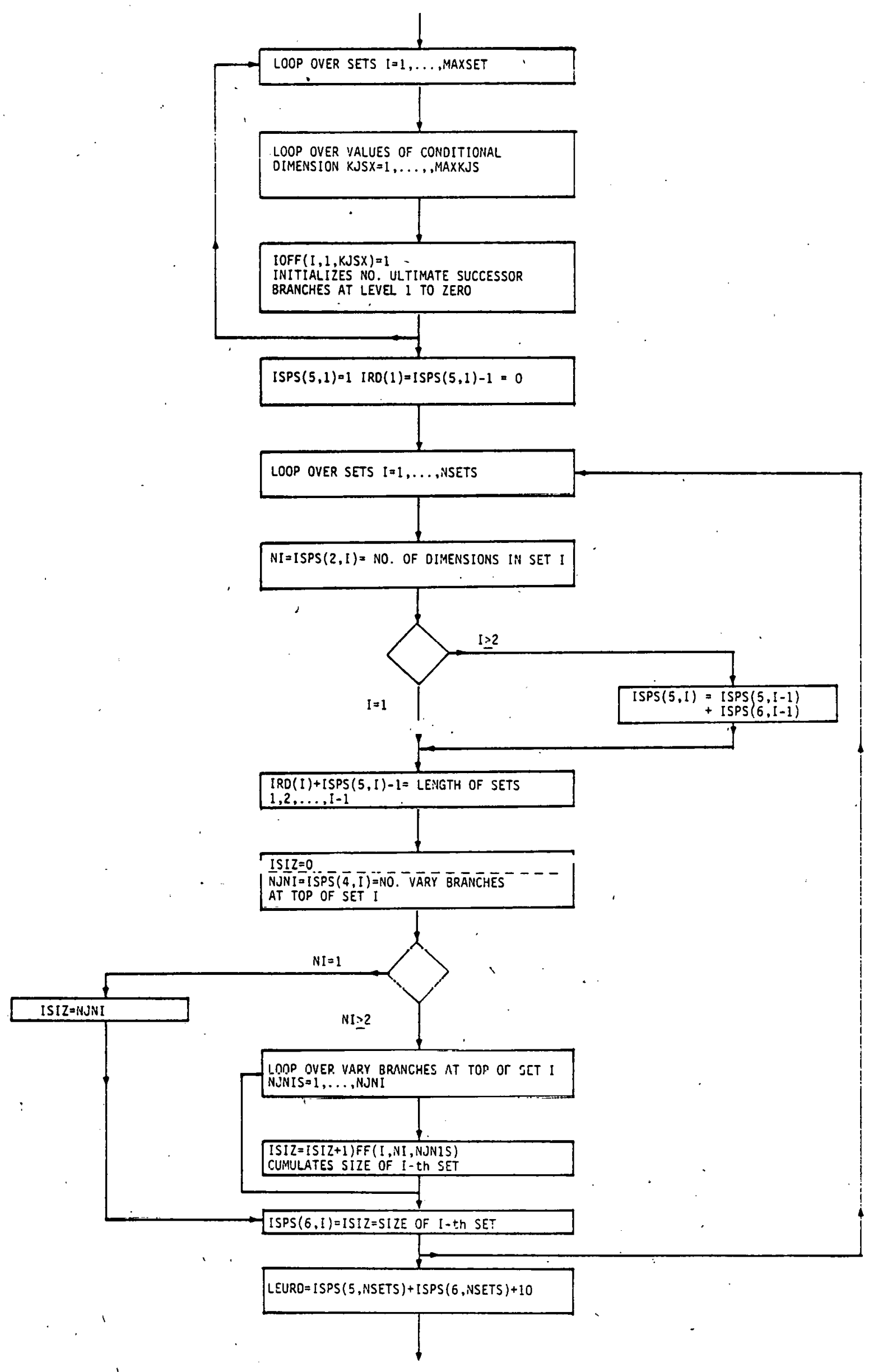

Figure VI.8.15: FINOFF CALCULATIONS 


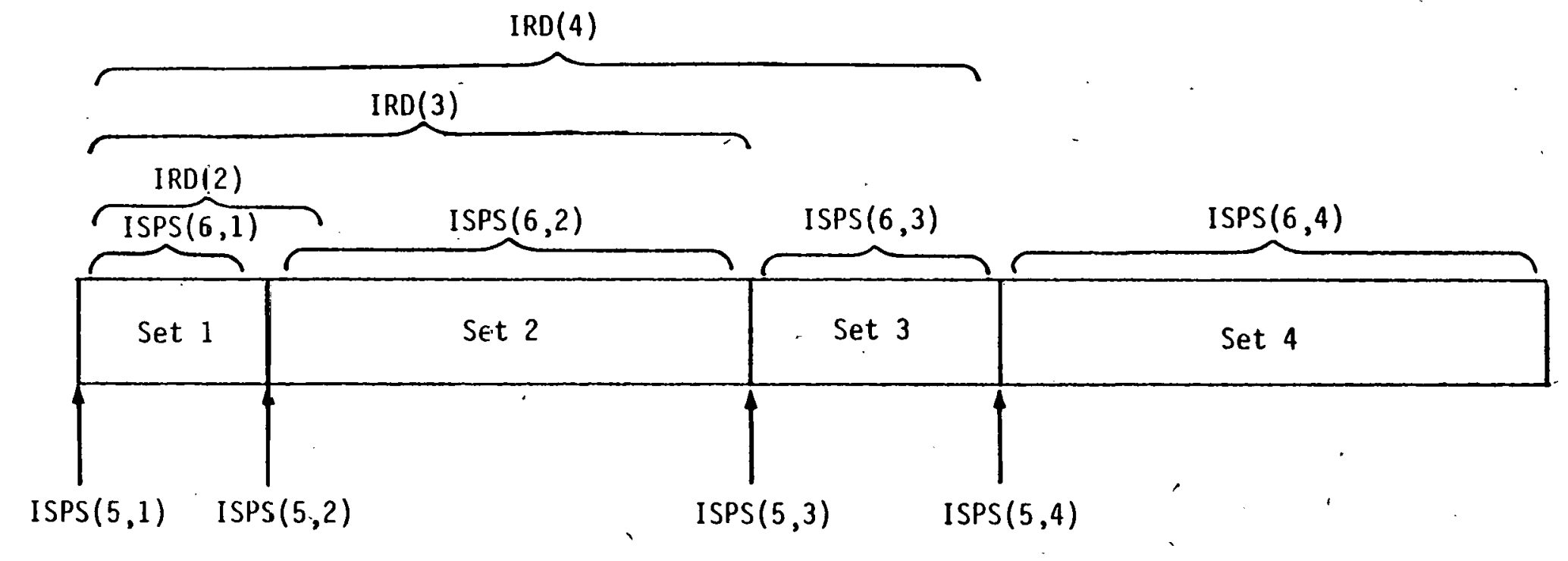

Figure VI.B.16: STRUCTURE OF THE RD VECTOR 
values associated with nodes at level 1 and placing them into the $R D$ vector.

Because we wish to traverse the same System 2000 unload file, we will use the same character reading algorithm that we described in the BLDOFF/OCOMP section with the minor modification that we will not ignore numerical data values associated with element numbers at level 1.

Figure VI.B.17 illustrates the algorithm used to read the system 2000 unload file. The reader will note the similarity with the algorithm in Figure VI.B. 8 except that now we have added two new functions:

$$
\begin{aligned}
& \text { DIDATA: reads the integer value, places it into } \\
& \text { the } R D \text { vector in the IRD(IS) }{ }^{\text {th }} \text { position, } \\
& \text { sets mode }=5 \text {, and returns. } \\
& \text { DRDATA: reads the real value, places it into the } \\
& \text { RD vector in the IRD(IS) })^{\text {th }} \text { position, sets } \\
& \text { mode }=5 \text {, and returns. }
\end{aligned}
$$

The pointer IRD(IS) will be described below. It will contain the pointer to the next available position in the RD vector for the IS th set.

When a mode $=5$ character string has been read in, we move to (B) in the figure and call DCOMP.

Subroutine. DCOMP is quite similar to OCOMP in terms of logic flow and function. We shall describe the front end section quickly, 


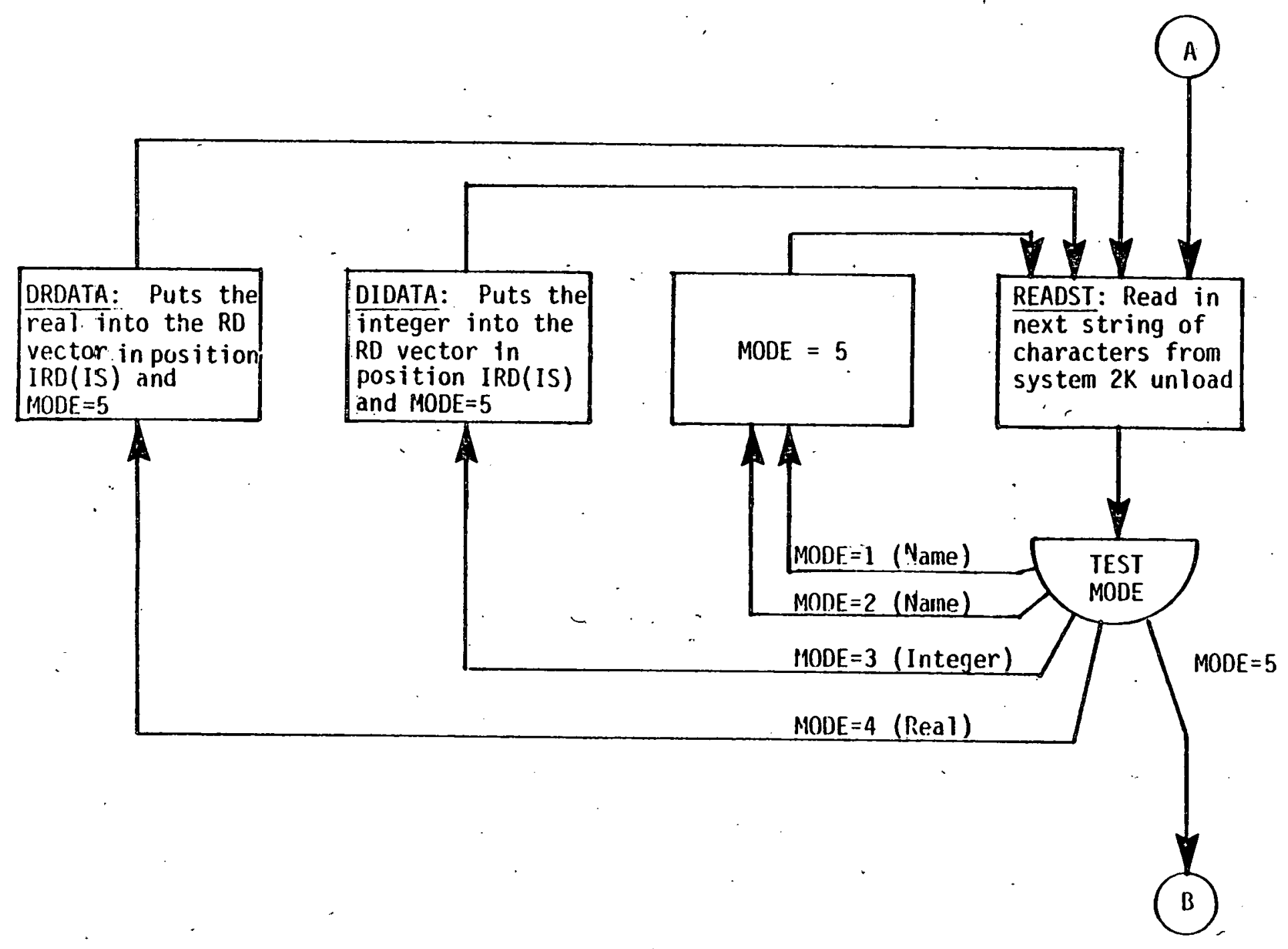

Figure VI.B. 17 READING CHARACTER STRINGS IN BLDD 
as it is identical to that in OCOMP, and proceed onward to the tree traversal algorithm. The logic in the front end of DCOMP is identical with that of OCOMP; the reader is referred to the previous section for a discussion.

When we have reached point (C) in the flow chart, we will begin the tree traversal portion of the BLDD algorithm. Figure VI.B.19 contains the flow chart for the tree traversal agorithm. Because we are "retracing our steps" through OCOMP, we have the same three cases regarding the node we just left and the node we just entered:

Case 1: JH $<$ JHOLD - - we have moved down the tree

Case 2: JH=JHOLD -- we have moved across the tree

Case 3: JH > JHOLD -- we have moved up the tree

These three cases will be explained in varying degrees of detail depending on their similarity to the OCOMP aigorithm.

Case 1: In this case we set $\operatorname{IDV}(J H)$ to 1 if $J H=1$ and 0 if $J H \geq 2$. Next we determine whether $J H=1$, in which case we have just come down the leftmost attribute branch. If $\mathrm{JH}=1$, we determine the set IS containing the current path IDV down to level 1. Given this set index, we add $I$ to the pointer IRD(IS) to reserve a place for the value we are about to read in:

$$
\operatorname{IRD}(I S)=\operatorname{IRD}(I S)+1
$$

Recall that IRD(IS) is the pointer to the next position in the RD vector to be filled in the portion of $R D$ corresponding to the Is ${ }^{\text {th }}$ 


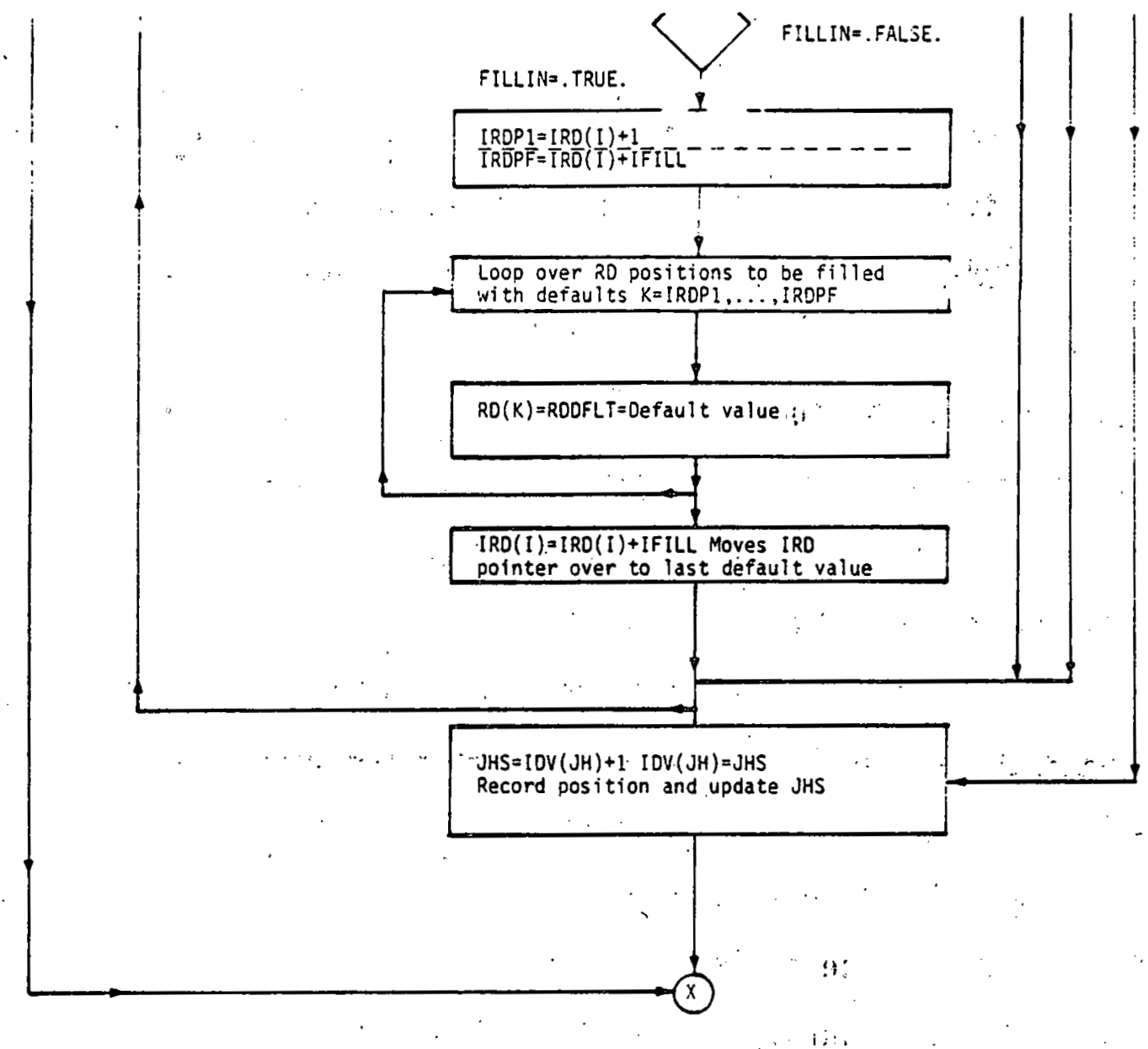

Figure VI.8.18: TRAVERSING THE HIERARCHICAL TREE IN OCOMP 
Figure VI.B. 18 (Top Left)

(c)

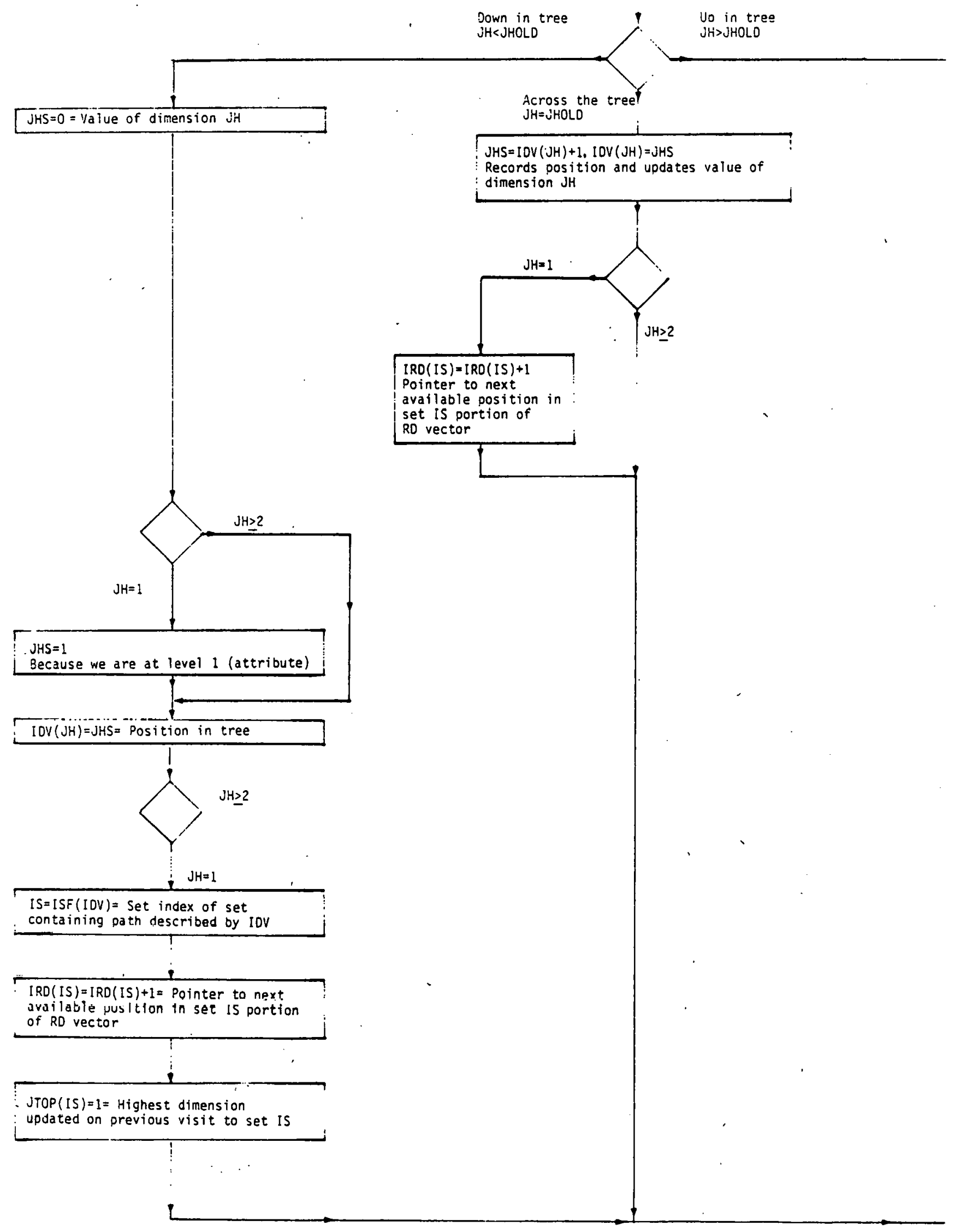




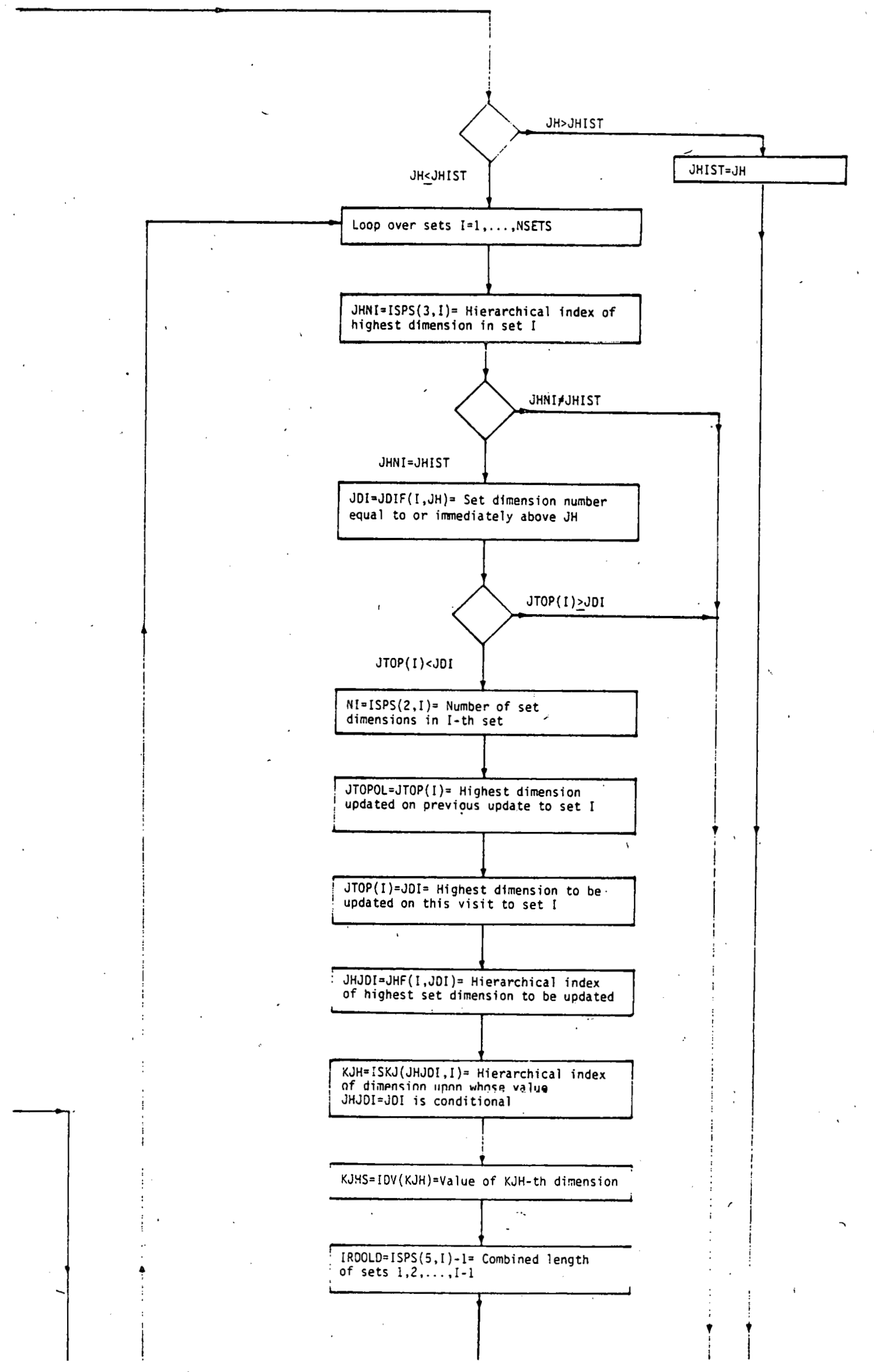




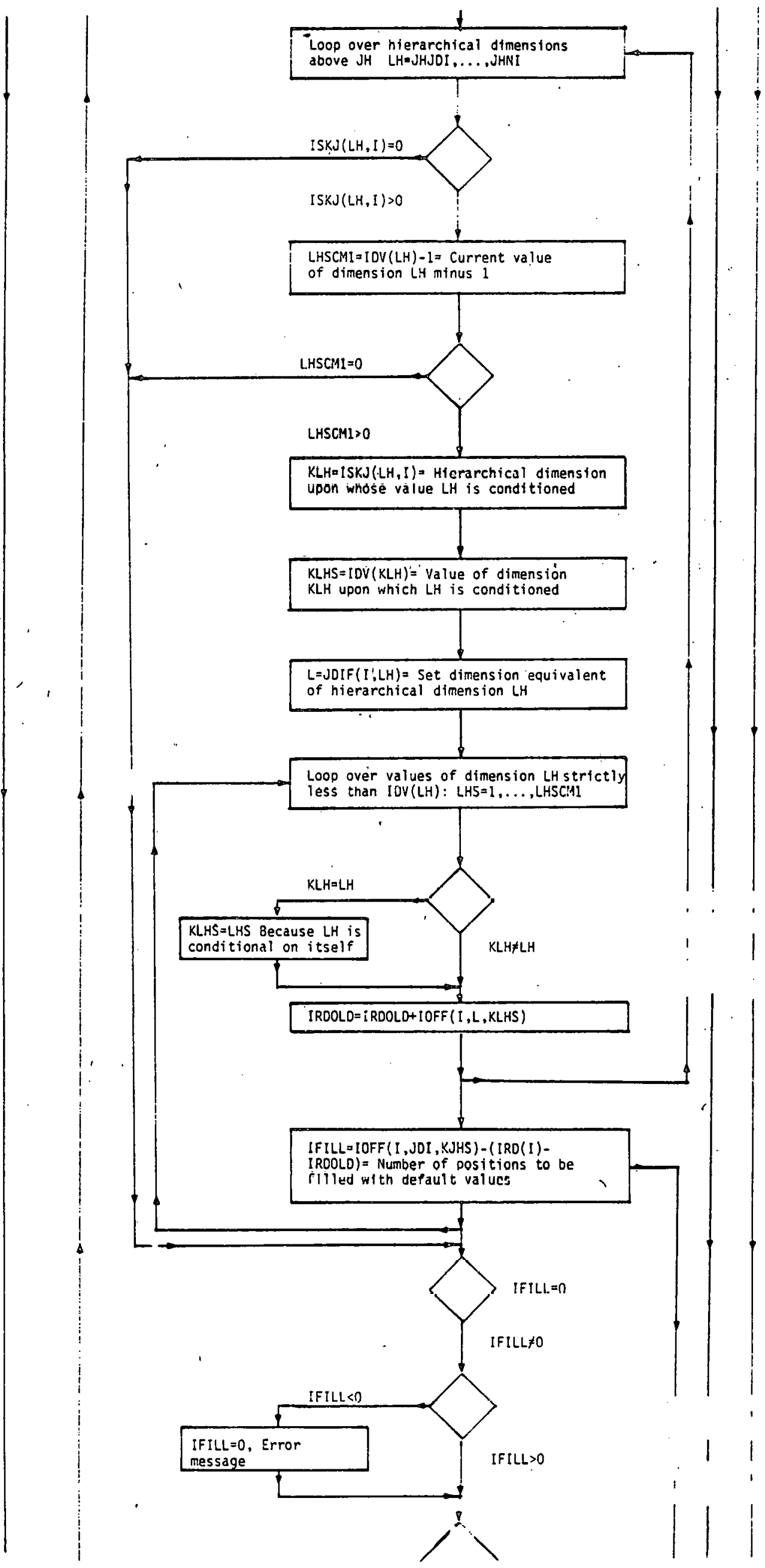


set. (This algorithm only works if there are no "placeholder" branches in the tree.) Finally, we initialize JTOP(IS) to 1 , indicating that on our most recent visit to set IS we hit the bottom of the tree. After these computations we go to $\chi$ and read the next value.

Case 2: In this case, we have moved across the tree to the next sibling at the $J H^{\text {th }}$ level. If $J H O L O=J H \geq 2$, we know that the old node had no data and was either a "placeholder" or a null node. If on a subsequent node visit we jump down before jumping up, we know it was a placeholder. If we jump up before jumping down, it was a null node. In this "across" case, we need only add 1 to the current value of JHS at level JH and 1 to the current value of IDV(JH) to record our new position in the tree. If $\mathrm{JH}=1$, we must reserve a position in the RD vector for the value that is about to be read in. Thus, we increment the pointer for the ISth set:

$$
\operatorname{IRD}(I S)=\operatorname{IRD}(I S)+1
$$

and go to $X$. to get a new value from the system 2000 unioad file. Case 3: In this case, we must move over in the RD vector to the first storage position corresponding to the new IDV vector at level JH. In other words, we have moved the IDV vector from its old value : 


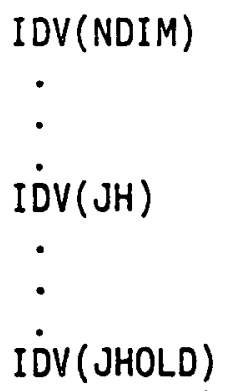

to its new value:

$$
\begin{aligned}
& \operatorname{IDV}(\text { NDIM) } \\
& \vdots \\
& \operatorname{IDV}(J H)+1
\end{aligned}
$$

This forces us to move over a number of positions in the RD vector and to fill those positions with default values. The algorithm for doing this was outlined in Chapter III.

The first step is to test whether the new node is higher than the previous highest jump JHIST. If JH> JHIST, we know that we are finished with all sets whose highest dimension is JHIST and we must begin with all sets whose highest dimension is $\mathrm{JH}$. Thus, in this case, we set JHIST $=\mathrm{JH}$, record our position in the tree, and return to BLDD. We know that by so doing we will not miss a value since they all occur at level 1 and we jumped up to $\mathrm{JH} \geq 2$.

In the event that JH $\leq$ JHIST, we know that we have jumped up to a level in the tree that is less than or equal to the top dimension JHIST of all open sets. Thus, we have finished loading values into some conditional fans in the tree and we must read default values into the empty storage positions. 
Because the early calculations in DCOMP are similar to those in OCOMP, we will summarize them breifly. We set up a loop over sets $I=1, \ldots$, NSETS and compute.

$$
\begin{aligned}
\text {, } \operatorname{JHN} I=\operatorname{ISPS}(3, I)= & \text { hierarchical index of highest } \\
& \text { dimension in set } I .
\end{aligned}
$$

We then teșt whether set I is open (JHNI=JHIST) and if so, we compute

$$
\begin{aligned}
\operatorname{JDI}=\operatorname{JDIF}(I, J H)= & \text { set dimension number equal to or } \\
& \text { immediately above } \mathrm{JH} .
\end{aligned}
$$

We then test whether JTOP(I) $\geq J D I$, in which case we have previously filled the default positions in the I th set. If JTOP(I)< JOI, we know that the previous jump up in the $I$ th set did not reach dimension JDI and thus we must fill in some null values in the RD vector. In this case, we compute

$$
\begin{aligned}
& N I=I S P S(2, I)=\text { number of set dimensions in } I^{\text {th }} \text { set } \\
& \text { JTOPOL }=\operatorname{JTOF}(I)=\text { highest dimension updated on previous } \\
& \text { visit to set I } \\
& \operatorname{JTOP}(I)=J D I=\text { highest dimension updated on this visit } \\
& \text { in set I } \\
& J H J D I=J H F(I, J D I)=\text { hierarchical index of highest set } \\
& \text { dimension updated this visit to } \\
& \text { set I }
\end{aligned}
$$




$$
\begin{aligned}
& \mathrm{KJH}=I S K J(J H J D I, I)=\text { hierarchical dimension upon } \\
& \text { whose value JDIth set dimension } \\
& \text { is conditional } \\
& K J H S=\operatorname{IOV}(K J H)=\text { value of } K J H^{\text {th }} \text { dimension } \\
& \operatorname{IRDOLD}=\operatorname{ISPS}(5, I)-1=\text { combined length of sets } \\
& 1,2, \ldots, I-1 \text {. }
\end{aligned}
$$

After completing these preliminary calculations, we will place the necessary default values into the RD vector to account for excess storage at dimensions JDI, ..., ISPS $(3, I)=J H J D I, \ldots, J H N I$.

We note that we have moved from

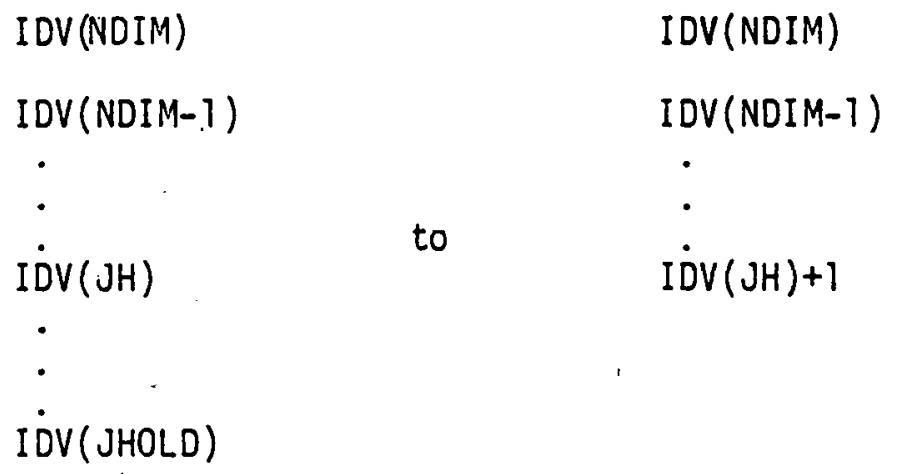

In order to determine how many default values would have to be inserted in the RD vector as a result of this jump, we make the following calculations

$$
\begin{aligned}
\text { IRDOLD+1 } & =\text { pointer to first storage position for } \\
& \text {, the IDV vector }
\end{aligned}
$$

$\operatorname{IDV}(N D I M)$ $6-56$ 


$$
\begin{gathered}
\operatorname{IDV}(\mathrm{JH}) \\
\operatorname{IDV}(\mathrm{JH}-1)=0 \\
\operatorname{IDV}(2)=0 \\
\operatorname{IDV}(1)=1, \\
\text { IOFF(I, JDI; IDV(KJH)) where KJH=ISKJ(JHJDI, IS) }= \\
\text { dimension upon which JDI th dimension is } \\
\text { conditional. This quantity is the space } \\
\text { allocated to store values at level JDI in } \\
\text { set I. }
\end{gathered}
$$

Hence, IRDOLD + IOFF (I,JDI, IDV $(K J H))$ is the last position in the RD vector where values for dimension JDI in set I are stored. We can think of IRDOLD+IOFF(I,JDI,IDV $(K J H))$ as the pointer to the last position prior to the position corresponding to

$$
\begin{aligned}
& \operatorname{IDV}(\text { NOIM) } \\
& \operatorname{IDV}(J H)+1 \\
& \operatorname{IDV}(J H-1)=0 \\
& \operatorname{IDV}(2)=0 \\
& \operatorname{IDV}(1)=1
\end{aligned}
$$

We also know that IRD(I) is the position in the RD vector of the last 
value that was stored. Thus, we have the situation shown in Figure VI.B.20. We know that the number of default values to be added is

$$
I F I L L=I O F F(I, J D I, I D V(K J H))-(I R D(I)-I R D O L D)
$$

Thus, our only task given this upward jump is to compute IRDOLD and then to compute IFILL. In order to do so, we begin by establishing a loop over hierarchical dimensions LH=JHJDI,..., JHNI. We then test whether $\operatorname{ISKJ}(L H, I)>0$. If not, we ignore dimension LH. If so, we then compute

$$
\operatorname{LHSCM}=\operatorname{IOV}(\mathrm{LH})-1
$$

and test whether $\operatorname{IDV}(L H)=1$, i.e., if $\operatorname{IOV}(\mathrm{LH})-1=\operatorname{LHSCMI}=0$. If so, we know we are at the first value of LH and do not have to add any offsets to IRDOLD for that dimension. Thus, if LHSCMI $=0$, we ignore the $L H^{\text {th }}$ dimension. If LHSCMI >0, we must compute.

$$
\begin{aligned}
K L H=I S K J(L H, I)= & \text { hierarchical dimension upon whose } \\
& \text { value } L H \text { is conditiona } 1 \\
K L H S=I D V(K L H)= & \text { value of dimension } K L H \text { upon whose } \\
& \text { value } I H \text { is ennditional } \\
L=J D I F(I, L H)= & \text { set dimension equivalent of } \\
& \text { hierarchical dimension } L H .
\end{aligned}
$$

We then loop over all values of dimension $\mathrm{LH}$ from $1,2, \ldots$, IDV (LH) -1 i.e., $L H S=1, \ldots, L H S C M 1$. If $K L H=L H$, we know that dimension $L H$ is self conditional. If $K L H \neq L H$, dimension $L H$ is conditional on some other 


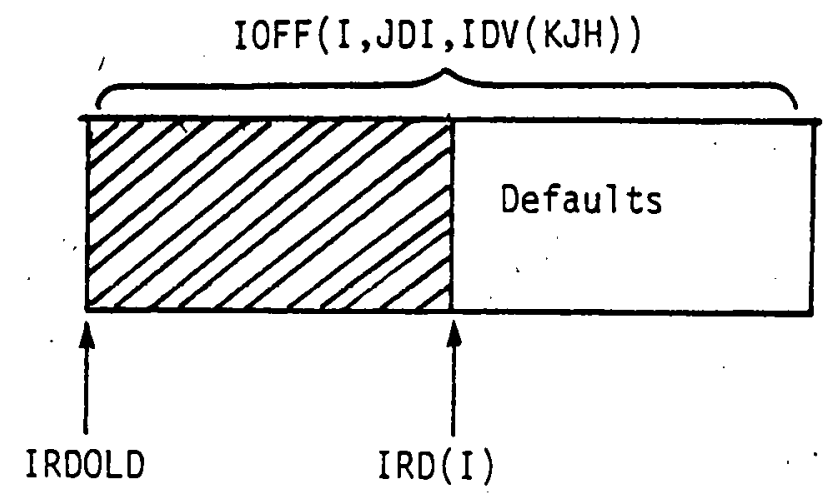

Figure VI.B.19 DEFAULT VALUES IN RD VECTOR 
nigher dimension. Hence, if $K L H=L H$, we use the offset

$$
\operatorname{IOFF}(I, L, K L H S)
$$

and if $K L H=L H$ we use the offset

$$
\operatorname{IOFF}(I, L, L H S)
$$

to compute the contribution to IRDOLD from the $L^{\text {th }}$ set dimension. Thus, in the former use

$$
I R D O L D=I R D O L D+\sum_{K L H S=1}^{\text {LHSCM1 }} \operatorname{OFF}(I, L, \bar{K} \text { LHS })
$$

and in the latter case

$$
\begin{aligned}
& I R D O L D=I R D O L D+\sum_{K L H S=1}^{L H S C M I} \operatorname{IOFF}(I, L, L H S) \\
& =I R D O L D+L H S C M I * I O F F(I, L, L H S)
\end{aligned}
$$

This algorithm is referred to in the code as the "double sum" algorithm. The underlying methodology was described in Chapter III. Given IFILL, we know that we can fill the interval

$$
\operatorname{IRD}(I)+1 \text { to } \operatorname{IRO}(I)+\operatorname{IFILL}
$$


with default values if IFILL $\geq 1$. Thus we compute

$$
\begin{aligned}
& I R D P I=I R D(I)+] \\
& I R D P F=I R D(I)+I F I L L
\end{aligned}
$$

and establish a loop over positions to be filled with defaults $K=I R D P 1, \ldots, I R D P F$. At each step, we insert

$$
\operatorname{RD}(K)=R D D F L T
$$

Upon completion of this calculation, we move the IRD pointer for set I over to the end of the present block of storage

$$
\operatorname{IRD}(I)=\operatorname{IRD}(I)+I F I L L
$$

and get the next higher set index $I+1$.

When we have completed this calculation, we will record our new position in the tree

$$
\begin{aligned}
& J H S=\operatorname{IDV}(J H)+1 \\
& \operatorname{IDV}(J H)=J H S
\end{aligned}
$$

and return to BLDD to get a new node in the hierarchical tree.

\section{FILL/STOR Algorithm}

The FILL and STOR algorithms are the basic means of communication between the model logic and the data base. The function of FILL is to retrieve a subset of the model data base and present it to the model logic. The function of STOR is the inverse -- to take intermediate or final model results and store them in the data base. 
As discussed previousiy, the procedure we have called SOFTIN accesses the user-specified data base, processes it, and places it into the vector we call RD. The three dimensional offset array $\operatorname{IOFF}(I, J, K J S)$ computed in SOFTIN and the user specified storage conditionality matrix $\operatorname{ISKJ}(I, J)$ allow us to take any position vector IDV and access the corresponding value stored in RD. Expressed in terms of the original data tree, given a position vector IDV, we can easily find the number residing at that terminal position in the tree.

Returning for a moment to our simple present value model in Chapter II, it requires more than just a single input -- a single terminal branch value. It requires as input a vector of cash flows over time and two scalars as seen in the simple trees discussed in Chapter II. We thius must develop a procedure for accessing each of the terminal values needed to make the model calculation. We can think of this procedure in terms of "looping over" all position vectors IDV that point to input values that the model needs and transferring those values into the model. This is precisely the function of the FILL algoritim.

Although in the simple present value example, there is only a single output (PV), there may in more complicated models be vectors .. or matrices of outputs. If we are to use the data tree to store and manage outputs as well as inputs, we must transfer these results back 
to the data base using the inverse of the FILL algorithm. This reporting of model results back to the data base we will call the STOR algorithm.

In discussing the FILL algorithin, we shall refer generously to the detailed flow charts: of subroutine FILL in Figures VI.C. 1 through VI.C. 6 .

1. Initialization Steps in FILL

The first calculations in FILL are the following (see the flow chart in Figure VI.C.1):

LCPBAS $=1$ plus the pointer to the first word in the present (LCI-th) labelled common IS = index of the set in which the present (LCI-th) labelled common is contained

NI = number of sat dimensions in the IS-th set

IRDBAS = pointer to the position in the $R D$ vector where storage for the IS-th set begins. IRDMAX = pointer to the position in the RD vector where storage for the IS-th set ends

MAXNIS = number of dependence branches for the highest level set dimension in the IS-th set, i.e. the width of the IS-th set tree at its highest level. 


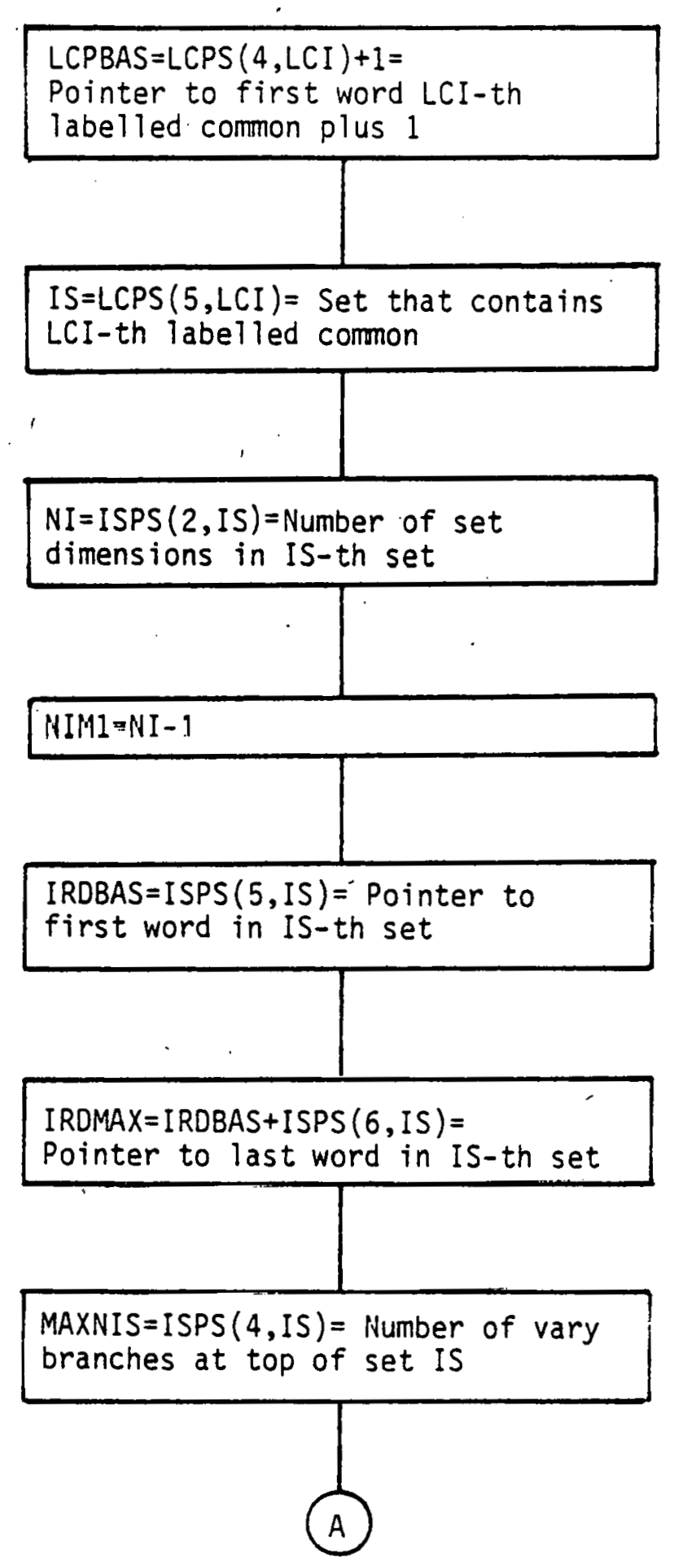

Figure VI.C.1: INITIALIZATION STEPS IN FILL 


\section{Filling From a One-Level Set}

Filling from a set containing only a single dimension is trivial and is treated as a special case in subroutine FILL for efficiency purposes as Figure VI.C.2 illustrates. If a set has only one dimension, we know by convention that the dimension is the attribute dimension. Furthermore, if the attribute dimension is fixed, we read in only one value. If it is free, we must read in $|\operatorname{LCS}(1, L C I)|$ values. As we can see in the flow chart, if $\operatorname{IDV}(1)>0$, dimension 1 is fixed and we simply set

$$
\begin{aligned}
& \operatorname{ALC}(L C P B A S+\operatorname{IDV}(1)-1) \\
& \text { position additional values } \\
& \text { of lst to get to IDV(1) } \\
& \text { value } \\
& =\operatorname{RD}(\operatorname{IRDBAS}+\operatorname{IDV}(1)-1) \\
& \text { position additional values } \\
& \text { of lst to get to IDV(1) } \\
& \text { value in in set IS } \\
& \text { set Is }
\end{aligned}
$$

If $\operatorname{IDV}(1)=0$ and $\operatorname{LCS}(1, \operatorname{LCI})$ is nonzero, we know dimension 1 is free and we must read in values $1, \ldots,|\operatorname{LCS}(1, L C I)|$. Hence, we simply set

$$
\begin{aligned}
& \text { ALC }(\underbrace{(L C P B A S}_{\text {position }}+\underbrace{J-1)}_{\text {additional values }} \\
& \text { of } 1 \text { st to get to } \operatorname{IDV}(1)=\mathrm{J} \\
& \text { value }
\end{aligned}
$$




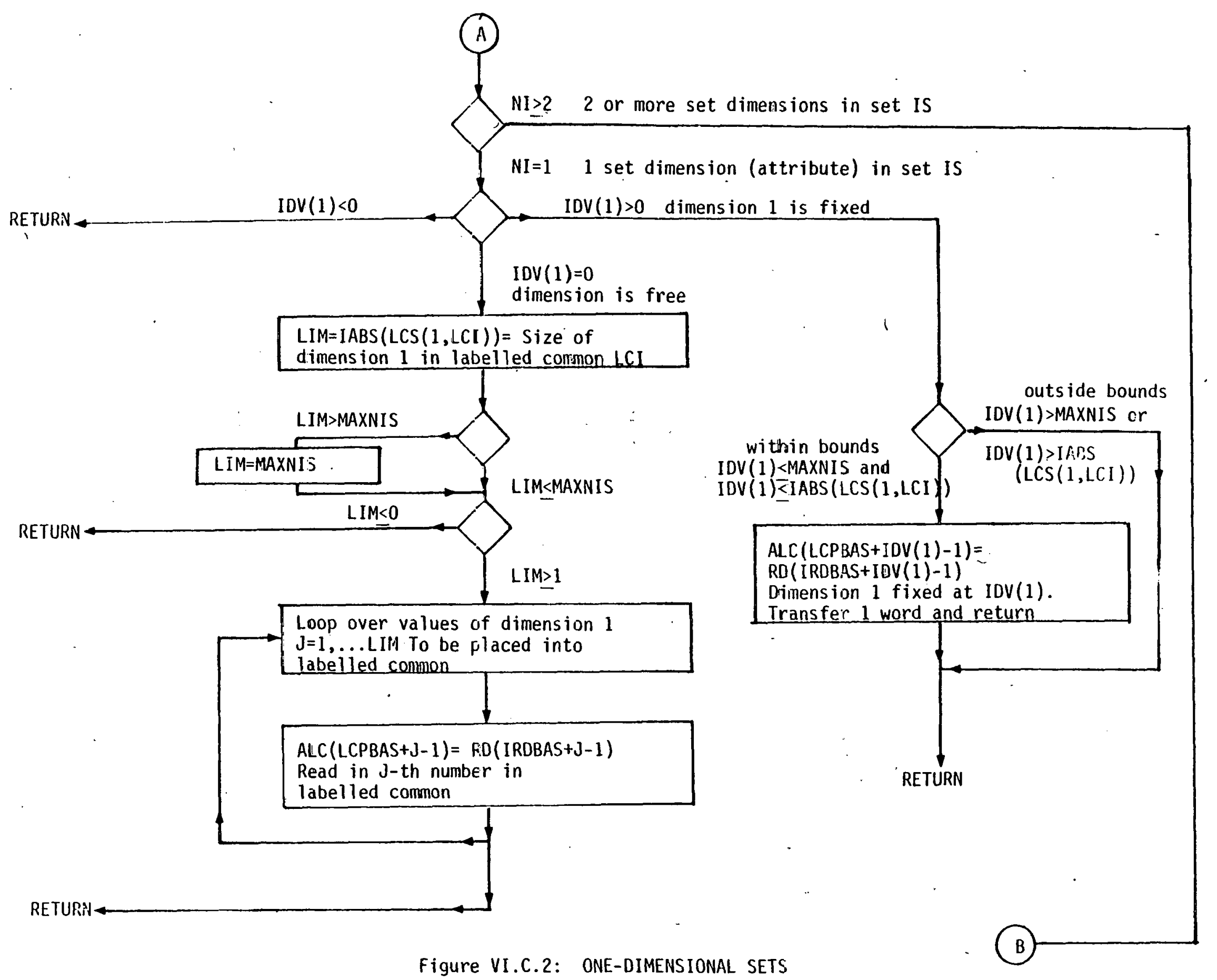




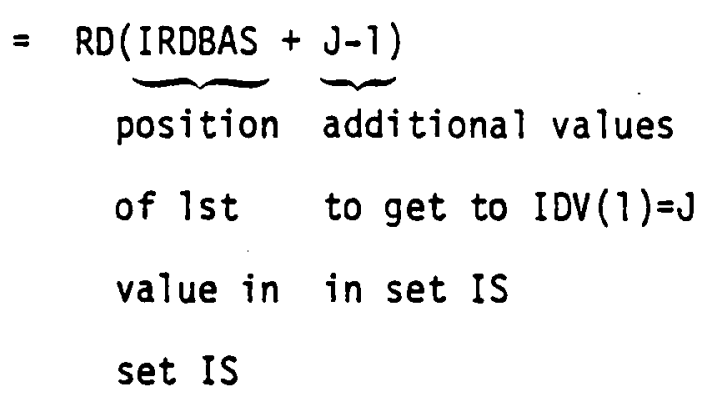

\section{Establishing the Clock}

In the case where there are two or more dimensions in a set and we wish to place numbers from that set into a labelled common, we begin by determining which dimensions in the set will have to be incremented to generate all the IDV vectors, which dimensions in the set will remain fixed, and which dimensions are not in the set and thus must be treated in a different fashion. This series of calculations is outlined in the flow chart in Figure VI.C.3.

We begin by establishing a loop over the hierarchical dimensions in hierarchical order from the bottom to the top of the hierarchical tree. Denote the index of this loop by $J$.

We then test whether both $\operatorname{LCS}(J, L C I)=0$ and $\operatorname{ISKJ}(J, I S)=0$ for the $J$ th dimension and the current (LCI ${ }^{\text {th }}$ ) labelled common. If $\operatorname{LCS}(J, L C I)=0$, we know that dimension $\mathrm{J}$ is not in the labelled common and if $\operatorname{ISKJ}(J, I S)=0$, dimension $\mathrm{J}$ is not in the set IS. Hence, if both occur, dimension $\mathrm{J}$ is in neither the labelled common nor the set and can be ignored in the "clock" algorithm to be discussed below. If either $\operatorname{LCS}(J, L C I) \neq 0$ or $\operatorname{ISKJ}(J, I S) \neq 0$, we know that dimension $J$ is in either the set IS or the labelled common LCI or both. 


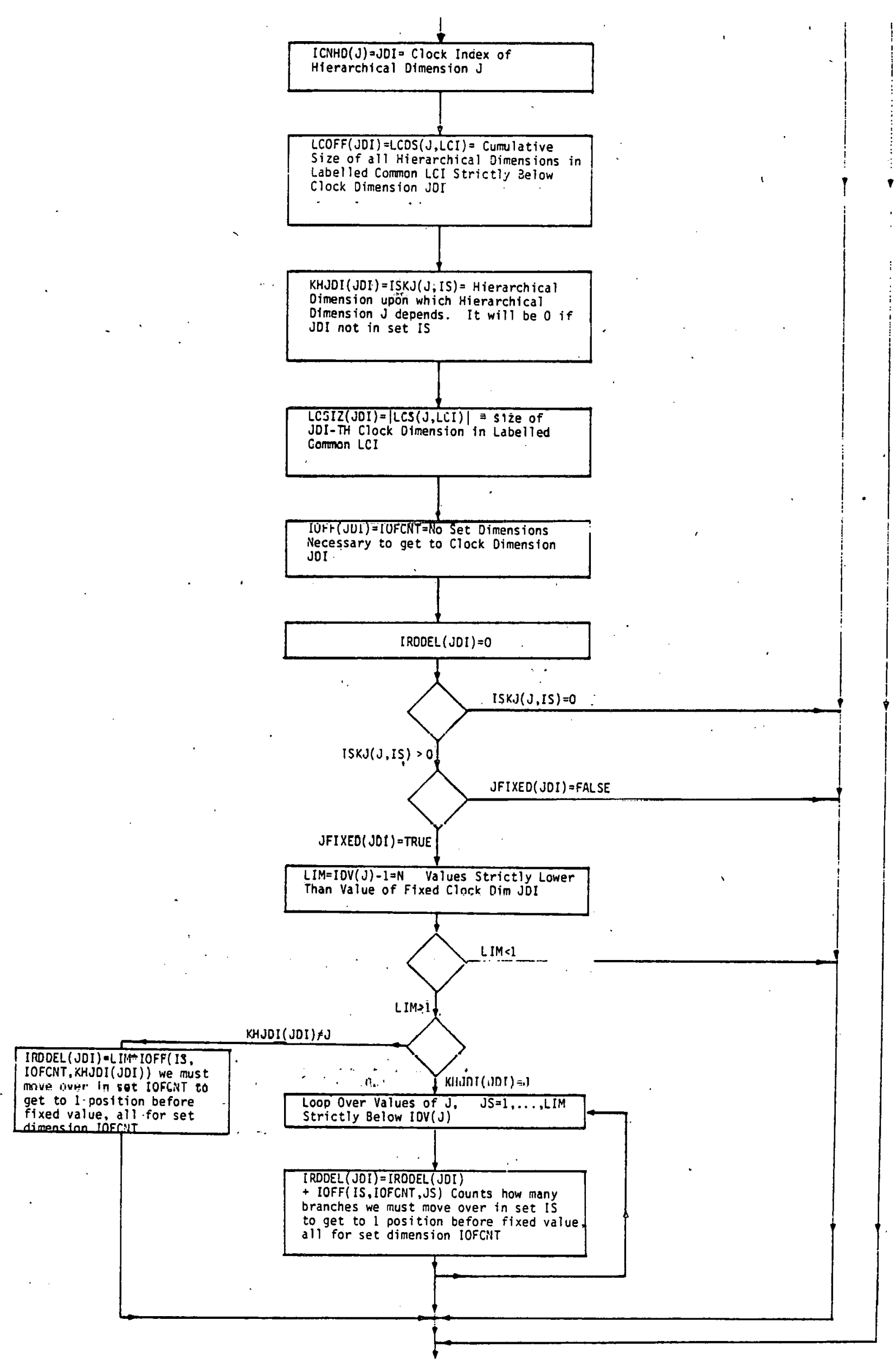

Figure VI.C.3: ESTABLISHING THE CLOCK 


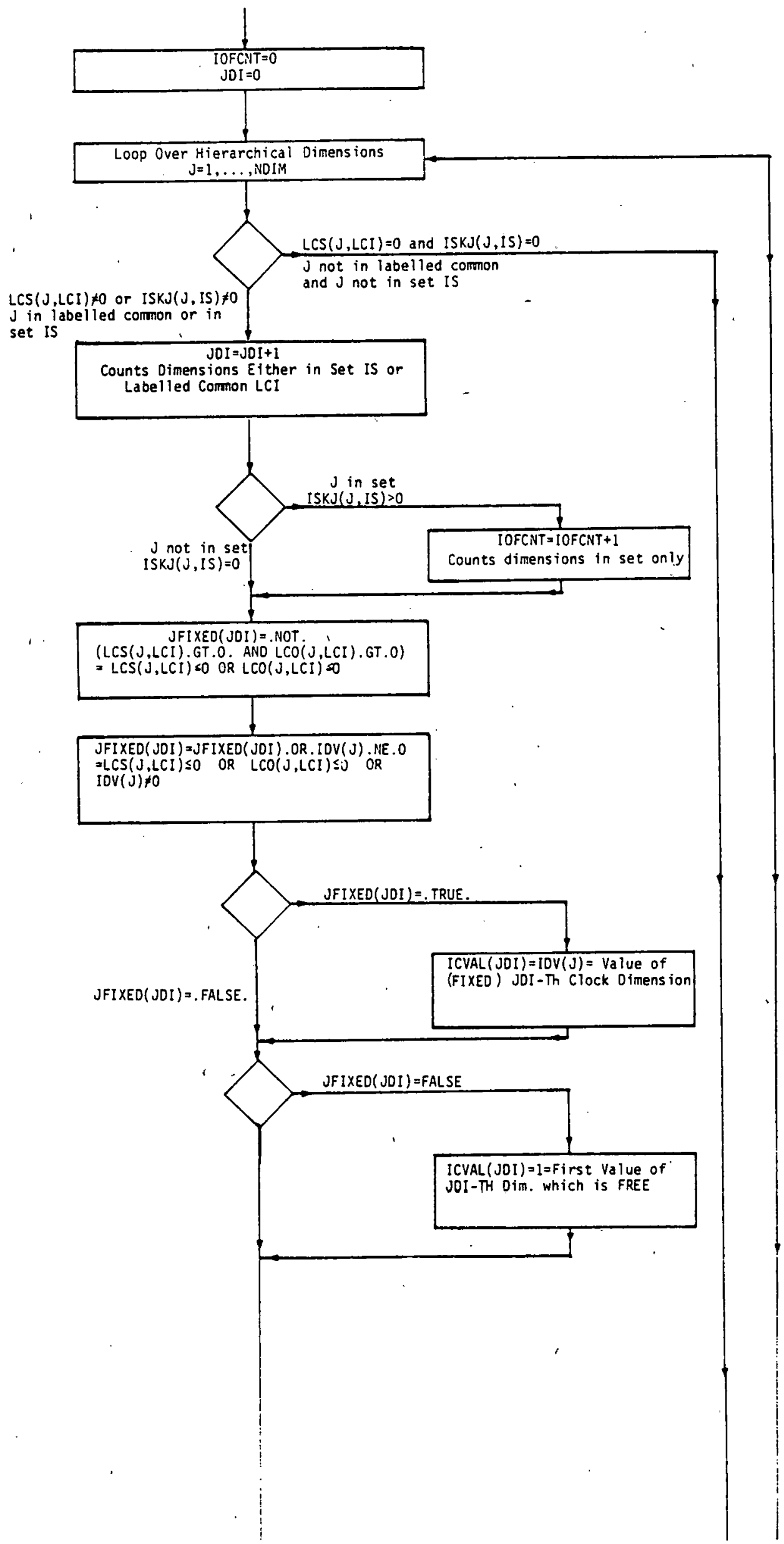


The next steps are to count the $\mathrm{J}^{\text {th }}$ dimension as a clock dimension and if it is in the ISth set to count it as a set dimension as well. The variable JDI contains the clock index and IOFCNT contains the set index of the present hierarchical dimension.

We next must determine whether the $J^{\text {th }}$ hierarchical $=$ JDI th clock dimansion wi: U wated is fixed or free. We have established the convention that the dimension, is fixed if one or more of the following user inputs is specified

1) $\operatorname{LCS}(J, L C I) \leq 0$

2) $\angle C O(J, L C I)=0$, or

3) $\operatorname{IOV}(J) \neq 0$

One of the above conditions will fix the $J^{\text {th }}$ hierarchical dimension within the clock at the value.10V(J). The truth table in Figure VI.C.4 contains the detailed criteria that are used to fix a dimension in the rabelled common. If the size of the $\mathrm{J}^{\text {th }}$ dimension in labelled common LCI is nonpositive, we assume dimension $\mathrm{J}$ lies outside set IS and is treated as fixed within the clock. If the order of the $J^{\text {th }}$ dimension in labelled common LCI is nonpositive, it is assumed to be fixed within the clock. Finally, if $\operatorname{IDV}(\mathrm{J})$ comes in as anything other than zero, its value will remain fixed at the value it came in with.

If the $k^{\text {th }}$ clock dimension is fixed, the value at which it has been fixed is placed into the array ICVAL. If the $k^{\text {th }}$ clock dimension is free, the array ICVAL is initialized to the first value 1 . To summarize 
$\operatorname{ISKJ}(I, J)>0$ : Dimension $J$ in Set I

\begin{tabular}{|c|c|c|c|c|c|c|}
\hline & \multicolumn{2}{|c|}{$\operatorname{LCS}(\operatorname{LCI}, \mathrm{J})<0$} & \multicolumn{2}{|c|}{$\operatorname{LCS}(L C I, J)=0$} & \multicolumn{2}{|c|}{$\operatorname{LCS}(\operatorname{LCI}, J)>0$} \\
\hline 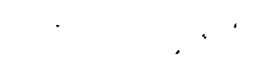 & $\operatorname{IDV}(\mathrm{J}) \neq 0$ & $\operatorname{IDV}(J)=0$ & $\operatorname{IDV}(J) \neq 0$ & $\operatorname{IDV}(\mathrm{J})=0$ & $\operatorname{IDV}(J) \neq 0$ & $\operatorname{IDV}(J)=0$ \\
\hline $\operatorname{LCO}(L C], J)<0$ & FIXED & FIXED & FIXED & FIXED & FIXED & FIXED \\
\hline $\operatorname{LCO}(\operatorname{LCI}, J)=0$ & FIXED & FIXED & FIXED & FIXED & FIXED & FIXED \\
\hline $\operatorname{LCO}(\operatorname{LCI}, \mathrm{J})>0$ & FIXE[ & FIXED & FIXED & FIXED & FIXED & FREE \\
\hline
\end{tabular}

$\operatorname{ISKJ}(I, J)=0$ : Dimension $J$ Not in Set $I$

\begin{tabular}{|c|c|c|c|c|c|c|}
\hline \multirow{2}{*}{-} & \multicolumn{2}{|c|}{$\operatorname{LCS}(\operatorname{LCI}, J)<0$} & \multicolumn{2}{|c|}{$\operatorname{LCS}(\operatorname{LCI}, \mathrm{J})=0$} & \multicolumn{2}{|c|}{$\operatorname{LCS}(\operatorname{LCI}, \mathrm{J})>0$} \\
\hline & $\operatorname{IDV}(\mathrm{J}) \neq 0$ & $\operatorname{IDV}(J)=0$ & $\operatorname{IDV}(J) \neq 0$ & $\operatorname{IDV}(J)=0$ & $\operatorname{IDV}(\mathrm{J}) \neq 0$ & $\operatorname{IDV}(\mathrm{J})=0$ \\
\hline $\operatorname{LCO}(\operatorname{LCI}, \mathrm{J})<0$ & FIXED & FIXED & IGNORE & IGNORE & FIXED & FIXED \\
\hline I. $C O(L C I, J)=0$ & FIXED & FIXED & IGNORE & IGNORE & FIXED & FIXED \\
\hline $\operatorname{LCO}(L C I, J)>0$ & FIXED & FIXED & IGNORE & IGNORE & FIXED & FREE \\
\hline
\end{tabular}

Figure VI.C.4 FILL TRUTH TABLE 


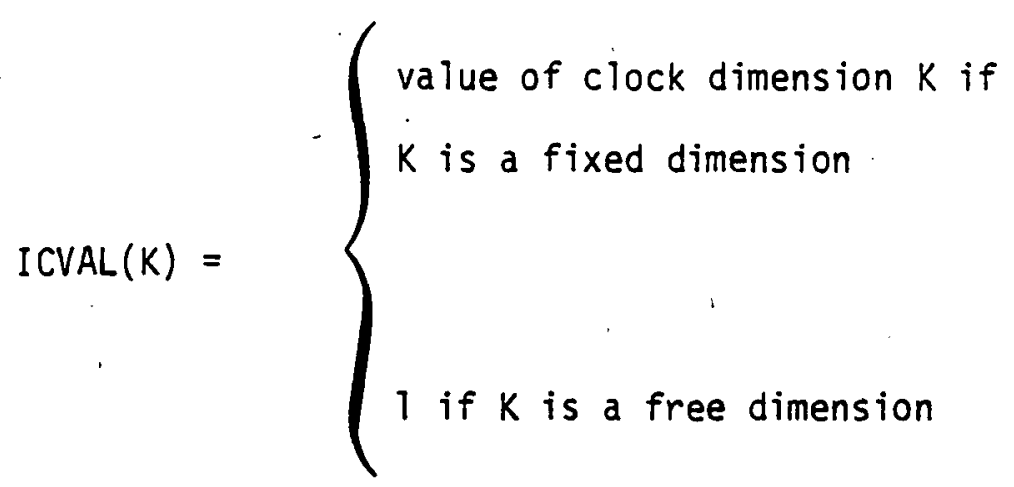

The next array to be initialized is $\operatorname{ICNHO}(\mathrm{J})$. This array will contain the clock index of hierarchical dimension $J$ if $J$ is in the clock. Repeating,

$$
\begin{aligned}
\operatorname{ICNHD}(J)= & \text { clock index of hierarchiral dimension } \\
& J \text { if it is in the clock. }
\end{aligned}
$$

If dimension $\mathrm{J}$ is not in the clock, the array ICNHD will contain no information.

We next compute the distance we must move in the labelled common to reach the point at which dimension $\mathrm{J}$ begins. Recall that this was precomputed in BLDTBL and placed into the array $L C D S(J, L C F)$.

Furthermore, the free dimensions are placed, in the order specified by the user, in the array $\operatorname{LCDS}(J, L C I)$. For example, if the user wishes to vary dimension 2 more rapidly than dimension 1, LCDS(J,LCI) will contain the entries $\left(0, s_{2}\right)$ in the first two positions, $s_{2}$ being the size of dimension 2 in the labelled common. Hence, the array LCDS(J,LCI) automatically takes care of the ordering of the IDV vectors (i.e., the ordering of the free dimensions) specified by the user in the labelled common order table. Technically, LCDS(J,LCI) contains the cumulative 
sizes of dimensions $1,2, \ldots, J-1$ in the labelled common. Thus,

$$
\begin{aligned}
\operatorname{LCOFF}(K)= & \text { cumulative size of all free hierarchical } \\
& \text { dimensions in the labelled common } \\
& \text { strictly below clock dimension } K .
\end{aligned}
$$

We next compute the dimension upon whose value storage for the $\mathrm{J}^{\text {th }}$ hierarchical $=$ JDI-th clock dimension is conditional. This is computed by simply transferring $\operatorname{ISKJ}(J, I S)$ into the vector KHJDI at the JDI-th position. Thus,

$$
\operatorname{KHJDI}(K)=\left\{\begin{array}{l}
\text { hierarchical dimension upon } \\
\text { whose value the } K^{\text {th }} \text { clock } \\
\text { dimension is conditional } \\
0 \text { if } K^{\text {th }} \text { clock dimension is } \\
\text { not in set IS }
\end{array}\right.
$$

We next obtain the size $|L C S(J, L C I)|$ of the $\mathrm{J}^{\text {th }}$ hierarchical dimension, which we know is the JDI th clock dimension. Using this information, we construct the array

$$
\begin{aligned}
\operatorname{LCSIZ}(K)= & \text { size of } K^{\text {th }} \text { clock dimension in labelled } \\
& \text { common } L C I .
\end{aligned}
$$

We next cumulate the number of set dimensions required to get to clock dimension $k$ if $k$ is in set Is or to the set dimension just below clock dimension $K$ if $K$ is not in set IS. Thus, 


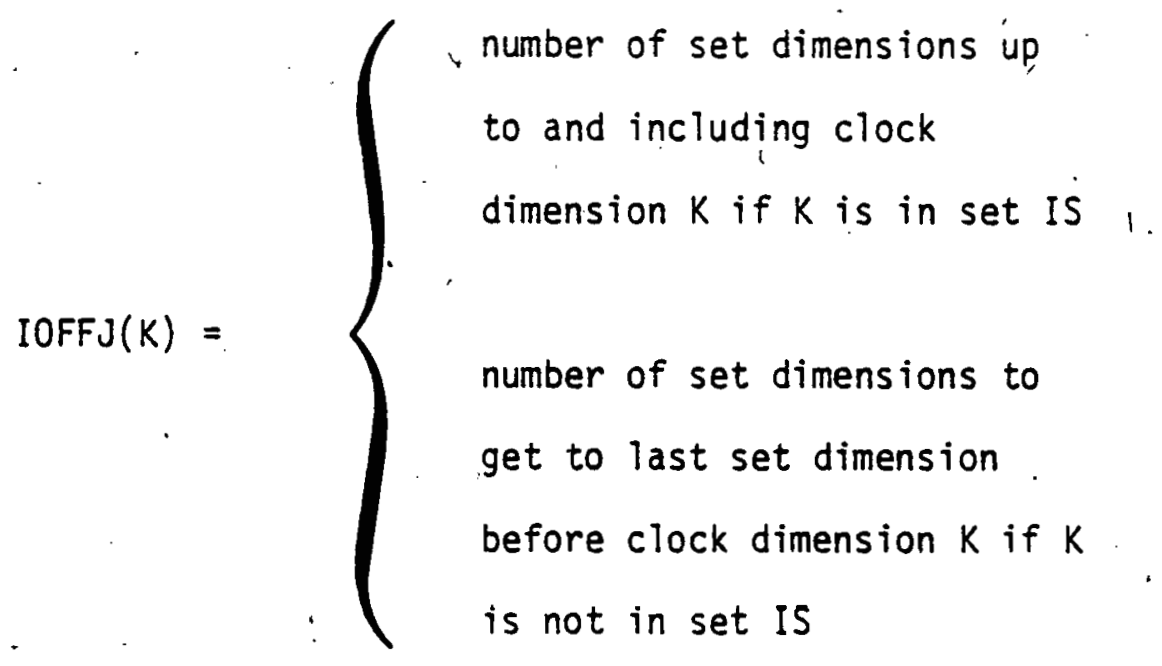

The last task in this section of the code is to set up an array IRDDEL $(K)$ which pre-computes the offset generated by the current value of dimension $K$ with respect, to the portion of the RD vector that stores set IS. In the event that clock dimension $K$ is not in set IS or clock dimension $K$ is free, IRDDEL $(K)$ is set to zero, indicating that we wish to begin at the first value of dimension $J$ in set IS. If dimension $K$ is fixed in labelled common LCI, we can pre-compute the offsets to be counted over to account for the values $1,2, \ldots, \operatorname{IDV}(J-1)$ of the fixed dimension $J$. The procedure is simple and depends on whether the offset for clock dimension $k$ is conditional on clock dimension $K$ or upon some higher level clock dimension. If clock dimension $K$ is "self-conditional", we set

$$
\operatorname{IRDDEL}(K)=\sum_{K J S=1}^{\operatorname{IDV}(K)-1} \operatorname{IOFF}(\operatorname{IS}, K, K J S)
$$


where it is understood that in IOFF and IDV we must really use the set dimension equivalent of clock dimension $k$. If clock dimension $k$ is conditional on some higher dimension, we know that the offsets at level $K$ are of equal length and thus,

$$
\operatorname{IRDOEL}(K)=[\operatorname{IDV}(K)-1] * \operatorname{IOFF}(I S, K, \operatorname{KHJDI}(K))
$$

Where again it is understood that in IDV and IOFF, we must use the set dimension equivalent of clock dimension $K$. When we complete these calculations and arrive at point (C) in the flow chart in Figure VI.B.3, we will have

$$
\operatorname{IRDOEL}(K)=\left\{\begin{array}{l}
0 \text { if } K \text { is not fixed } \\
\text { offset at set dimension } K \text { up } \\
\text { to but not including the value } \\
\text { of dimension } k \text { if } K \text { is fixed }
\end{array}\right.
$$

4. Establishing Conditionality Information For Clock Dimensions

In the next section of the code, we establish a loop over clock dimensions and develnp the clock indices of the dimensions upon which other clock dimensions are conditional. Let the index over clock dimensions be denoted J. Referring to Figure VI.C.5, we begin by testing whether clock dimension $\mathrm{J}$ is in the set.IS. If not, we do nothing -- that dimension is fixed. If so, we compute the clock index of the dimension upon whose value clock dimension $\mathrm{J}$ is conditional. 


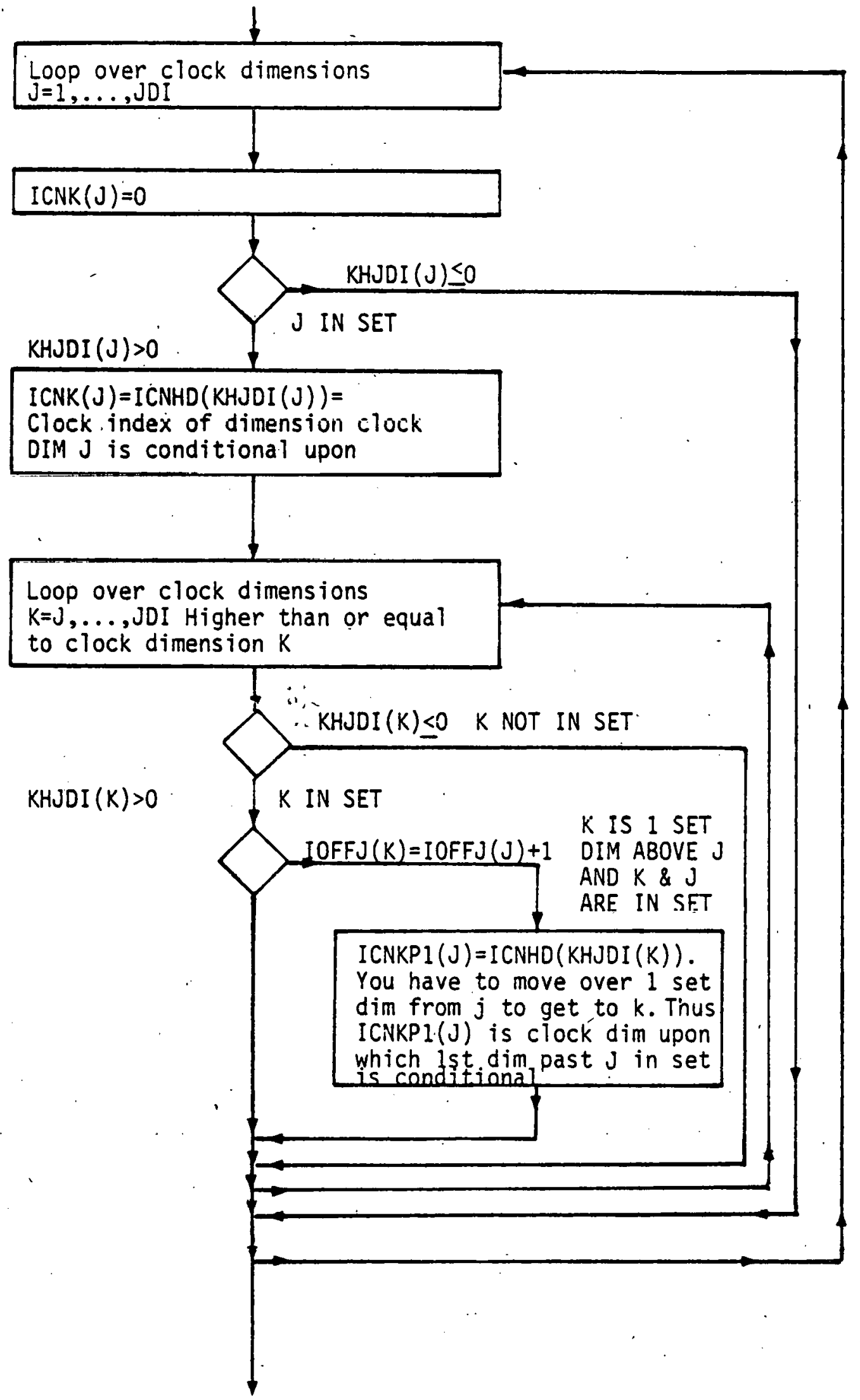

Figure VI.C.5: CONDITIONALITY INFORMATION FOR CLOCK DIMENSIONS 
That is, we compute the array

$$
\operatorname{ICNK}(J)=\left\{\begin{array}{l}
0 \text { if clock dimension } J \text { is not } \\
\text { in set IS } \\
\text { clock index of dimension upon } \\
\text { whose value clock dimension } J \\
\text { is conditional }
\end{array}\right.
$$

We then wish to find which dimension is the next higher set dimension above clock dimension $\mathrm{J}$. In order to do so, we simply find the clock dimension. $K=J, \ldots$, JDI for which

$$
\begin{array}{ll}
\underbrace{\operatorname{IOFFJ}(K)}_{\text {set dimension }} & =\underbrace{\operatorname{IOFFJ}(\mathrm{J})+1}_{\text {set dimension equivalent }} \\
\text { equivalent of } & \text { of clock dimension } \mathrm{J} \text { since } \\
\text { clock dimension } K & \mathrm{~J} \text { is in set IS }
\end{array}
$$

The dimension $K$ that satisfies this relationship is one above dimension $\mathrm{J}$ in set IS. We wish now to find the clock dimension upon whose value the $k^{\text {th }}$ clock dimension is conditinnal: We know that $\mathrm{KHJDI}(\mathrm{K})$ is the hierarchical dimension upon which clock dimension $\mathrm{K}$ is conditional. Dimension ICNHD[KHJDI $(K)]$ is the clock dimension equivalent of hierarchical dimension KHJDI(K). We store it in the array ICNKP] : 


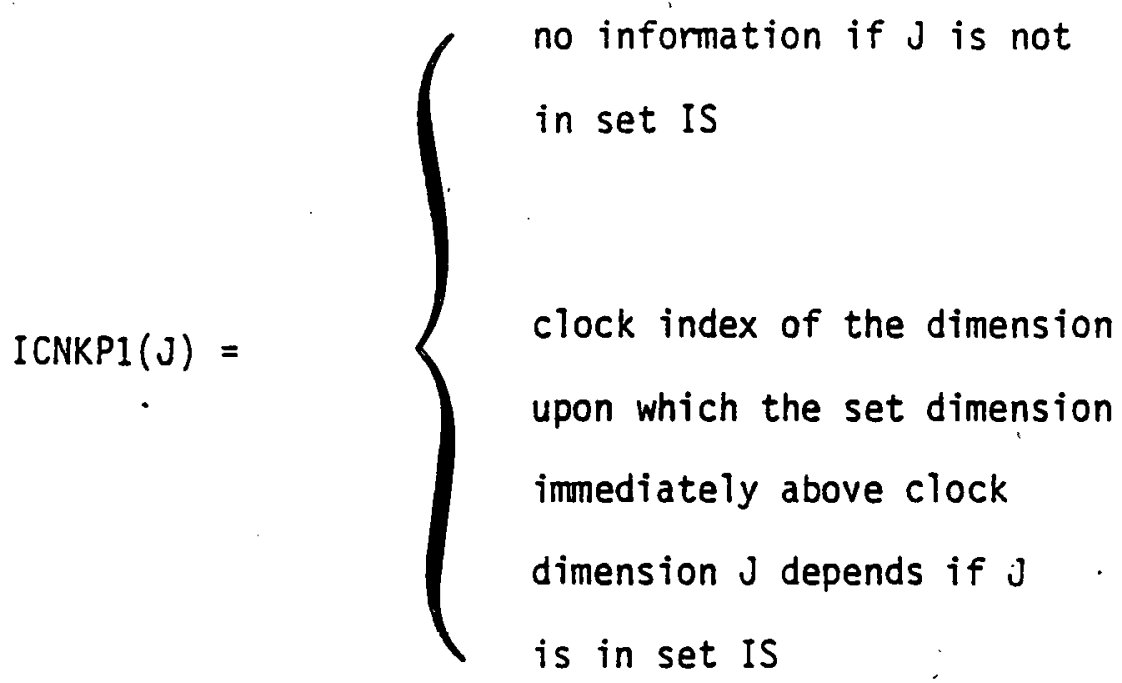

When we have completed these calculations, we have arrived at $(\bar{D})$ in the flow chart.

5. Determine Whether Any Values Need Be Read Into the Labelled Common Referring to the flow chart in Figure VI.C.6. we have performed only preliminary calculations up to the point (D) in the flow charts. Beginning at point (D) in Figure VI.C.6, we first must determine whether there are any values to be transferred into the labelled common.

We begin by establishing a loop over the dimensions in the clock $J=1,2, \ldots, J D I$. In the ensuing discussion, the reader will be able to see why we refer to the algorithm as a "clock".

The first test required for clock dimension $\mathrm{J}$ is to determine whether it lies in set IS or not. If not, we know it is a fixed dimension in the clock and we need only test whether the value assumed for that dimension lies within the size limit $\operatorname{LCSIZ(J)}$ for that 


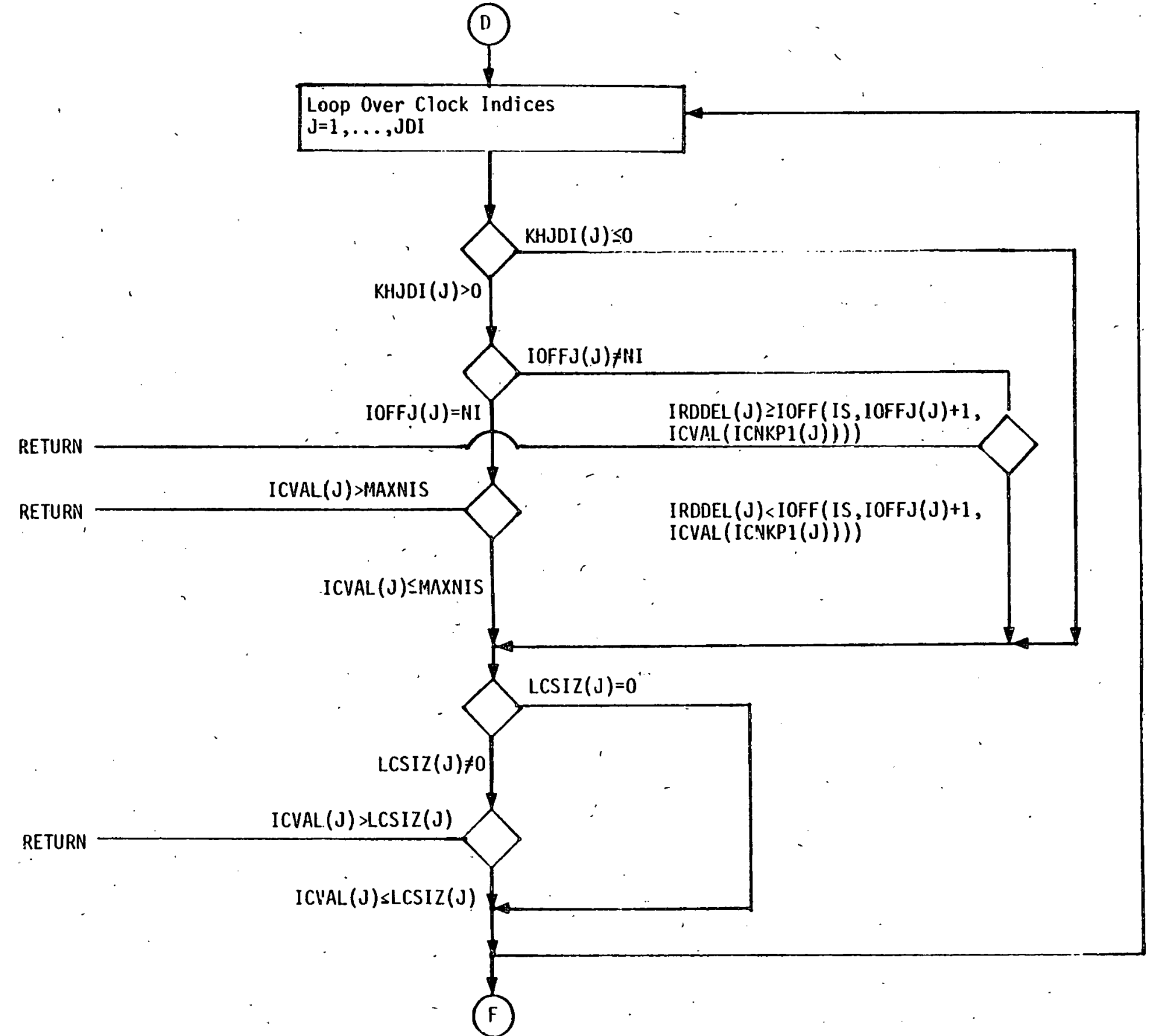

Figure VI.C.6: DETERMINE WHETHER ANY VALUES ARE TO BE TRANSFERRED 
dimension in the labelled common. If the value does not violate the bound, at least one value will be transferred. If the bound is violated, no values will be read, in.

If the dimension $\mathrm{J}$ does $\mathrm{lie}$ within set IS, we next test whether dimension $J$ is the highest set dimension in the set. If clock dimension $\mathrm{J}$ is the highest set dimension, we need only test whether its value lies within the size bound MAXNIS at the top of the set. If it violates the bound, no values are transferred. If the bound is not violated we test to see that the size bound is not violated in the labelled commons in the manner discussed just above.

If clock dimension $J$ is not the highest dimension in set IS, we must perform a different test to see whether the value assumed for dimension $\mathrm{J}$ violates the offset bounds at level $\mathrm{J}$. To do so, we. begin by noting that

$$
\begin{aligned}
\operatorname{IOFFJ}(J)+1= & \text { set dimension index of the set dimension } \\
& \text { immediately above clock dimension } \mathrm{J} \\
\operatorname{ICNKPI}(\mathrm{J})= & \text { clock index of the dimension upon which } \\
& \text { set dimension } \operatorname{IOFFJ}(J)+1 \text { is conditional } \\
\operatorname{ICVAL}(K)= & \text { value of clock dimension } k
\end{aligned}
$$

and hence, that

$$
\operatorname{IOFF}(\operatorname{IS}, \operatorname{IOFFJ}(J)+], \operatorname{ICVAL}[\operatorname{ICNKFI}(J)]) \cdot \ell(J)
$$

is the number of successor branches one set dimension above clock 
dimension $\mathrm{J}$ conditional on the value of one strictly. higher level dimension $\operatorname{ICNKPI}(\mathrm{J})$. Thus, $\ell(\mathrm{J})$ is the amount of storage allocated in the RD vector for storing values at the level of clock dimension $\mathrm{J}$ given $\operatorname{ICNKPI}(J)$. Thus, we know that the storage allocated for storage runs from indices

$$
\begin{aligned}
&(\text { IRDBAS- } 1)+1=\text { IRDBAS } \\
& \text { to } \quad(\text { IRDBAS- } 1)+\ell(J)
\end{aligned}
$$

Given a vector $\operatorname{IRDDEL}(\mathrm{J})$, the value being accessed is indexed

$$
\operatorname{IRDBBAS}+\operatorname{IRDDEL}(\mathrm{J})
$$

Hence, we know we have exceeded the storage bound at the $J$-th level if

$$
\operatorname{IRDBAS}+\operatorname{IRDDEL}(\mathrm{J})>\operatorname{IRDBAS}-\mathrm{I}+\ell(\mathrm{U})
$$

which can be reduced to

$$
\operatorname{IRDDEL}(J)>\ell(J)-1
$$

Since all terms are integers, this is equivalent to the condition

$$
\operatorname{IRDOEL}(J) \geq \ell(J) .
$$

Thus, we need only compare IRDOEL( $J)$ with the offset $\ell(J)$. If $\operatorname{IRDEL}(J) \geq \ell(J)$, we have violated the bound at lerel $\mathrm{J}$ and end. If $\operatorname{IRDDEL}(J)<\ell(J)$, we have not violated the bound at level $J$ and we can proceed. As before, we conclude by testing the size of clock 
dimension $\mathrm{J}$ to see that it is nonzero.

6. Transferring a Single Value From the RD Vector to the Labelled Common

The flow chart in Figure VI.C.7 is relevant to the present discussion. In order to transfer a single value from the RD vector to the labelled common, we must first determine the position of that value in the RD vector and then determine the proper position in the labelled common. We begin by discussing the RD vector.

Recall that IRDDEL( $(J)$ contains the number of storage positions we must move over in the portion of the RD vector where set IS is stored to account for the branch setting at the level of clock dimension $J$ in the set tree. In order to determine how far we must move over in total, we add IRDOEL(J) over all clock dimensions $\mathrm{J}$ :

$$
\sum_{J=1}^{J E I} \operatorname{IRDDEL}(J)
$$

We then simply add this number of positions to the pointer IRDBAS to the first position in the RD vector for set Is to obtain

$$
\operatorname{IRDBAS}+\sum_{j=1}^{J D I} \operatorname{IRDDEL}(J)
$$




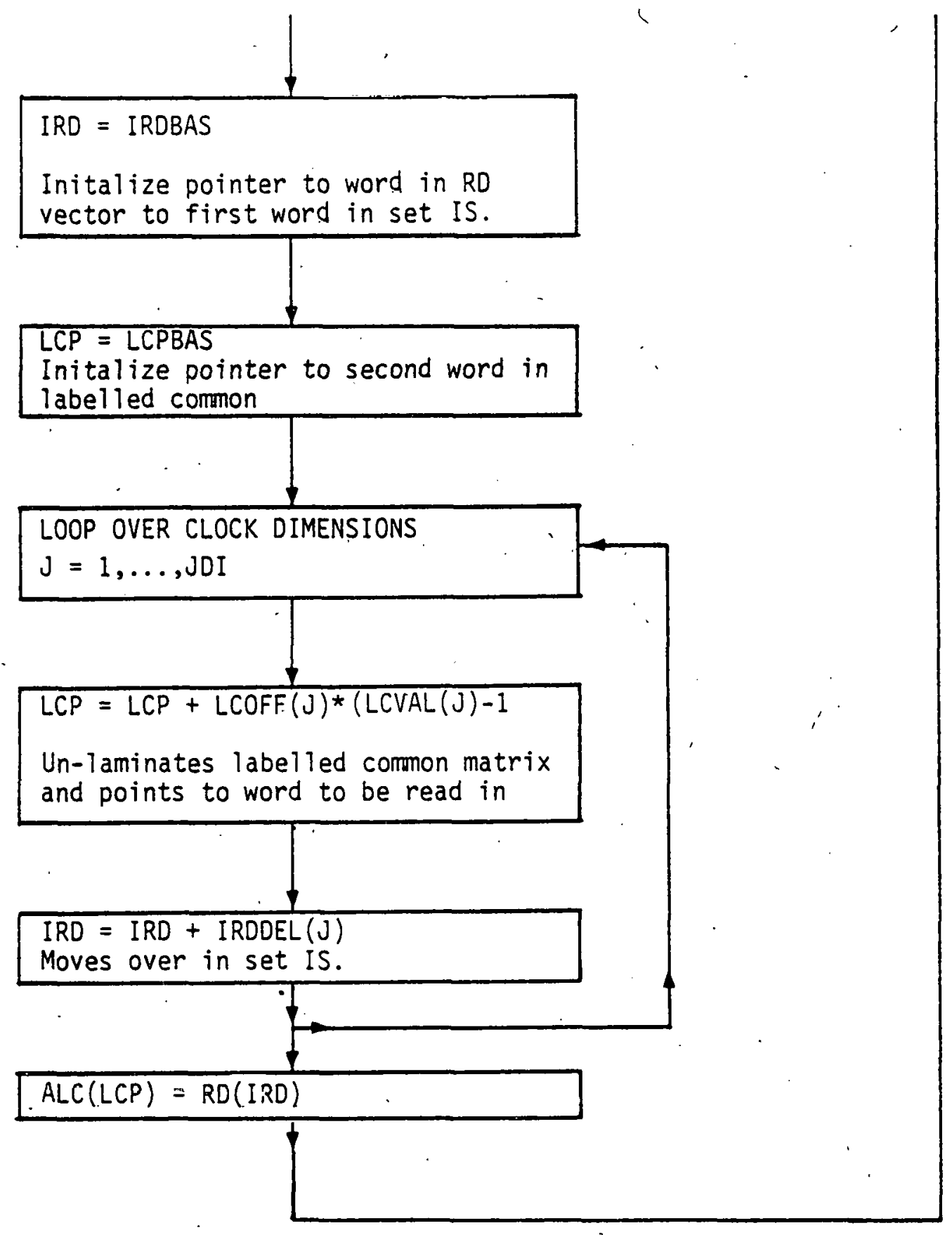

Figure VI.C.7: TRANSFERRING A SINGLE VALUE FROM THE RD VECTOR TO THE LABELLED COMMON 
which is the pointer to the appropriate position in the RD vector. The pointer to the appropriate position in the labelled common is obtained in a similar manner. We recall that $\operatorname{LCOFF}(J)$ contains the cumulative size in the labelled common of all dimensions that vary faster than the $J^{\text {th }}$ clock dimension. If dimension $J$ is fixed, $\operatorname{LCOFF}(\mathrm{J})$ is zero. Hence we know that

$$
\operatorname{LCOFF}(J) *[\operatorname{ICVAL}(J)-1]
$$

is the distance we must move over to account for the current value of dimension $\mathrm{J}$ in the clock. To see why, assume that dimensions $I$ and 2. vary and that $\operatorname{LCOFF}(1)=10$ and $\operatorname{LCOFF}(2)=0$, i.e. that dimension 2 varies more rapidly than 1 . If we wish to find where $\operatorname{IDV}(2)=3$, $\operatorname{IDV}(1)=4$ would be found, we would move over $4-1=3$ times the size of dimension 1 plus $3-1=2$ or $3 \times 10+2=32$ positions to get to the proper position in the labelled common. Hence, the pointer to the proper, position in the labelled common is

$$
L C P B A S+\sum_{J=1}^{J D I} \operatorname{LCOFF}(J) \times[\operatorname{ICVAL}(J)-1] .
$$

Thus, to place one value into the labelled common, we simply perform the operation 


$$
\begin{aligned}
& \operatorname{ALC}\left\{L C P B A S+\sum_{J=1}^{J D I} \operatorname{LCOFF}(J) \star[\operatorname{ICVAL}(J)-1]\right\} \\
& =\operatorname{RD}\left\{\operatorname{IRDBAS}+\sum_{J=1}^{J D I} \operatorname{IRDDEL}(J)\right\}
\end{aligned}
$$

7. Incrementing the Clock to Determine Whether to Transfer Another Value

The following discussion contains the essence of the "clock" algorithm. The reader is referred to the flow chart in Figure VI.C.8. The reason we refer to the algorithm as a "clock" algorithm is that we sweep through the IDV vectors in a fashion analogous to that in which a clock sweeps simultaneously through seconds, minutes, hours, days, months, and so forth.

We begin the clock algorithm setting the variable $J_{H I}$ to 1 . This variable will sweep through the clock dimensions from 1 to JDI picking out the free clock dimensions in order and incrementing or resetting them as appropriate.

After resetting JHI to 1 , we first test whether JDI<JHI, in which case we have exceeded the highest clock dimension in the labelled common and can end. If JDI $\geq \mathrm{JHI}$, we must test whether clock dimension 


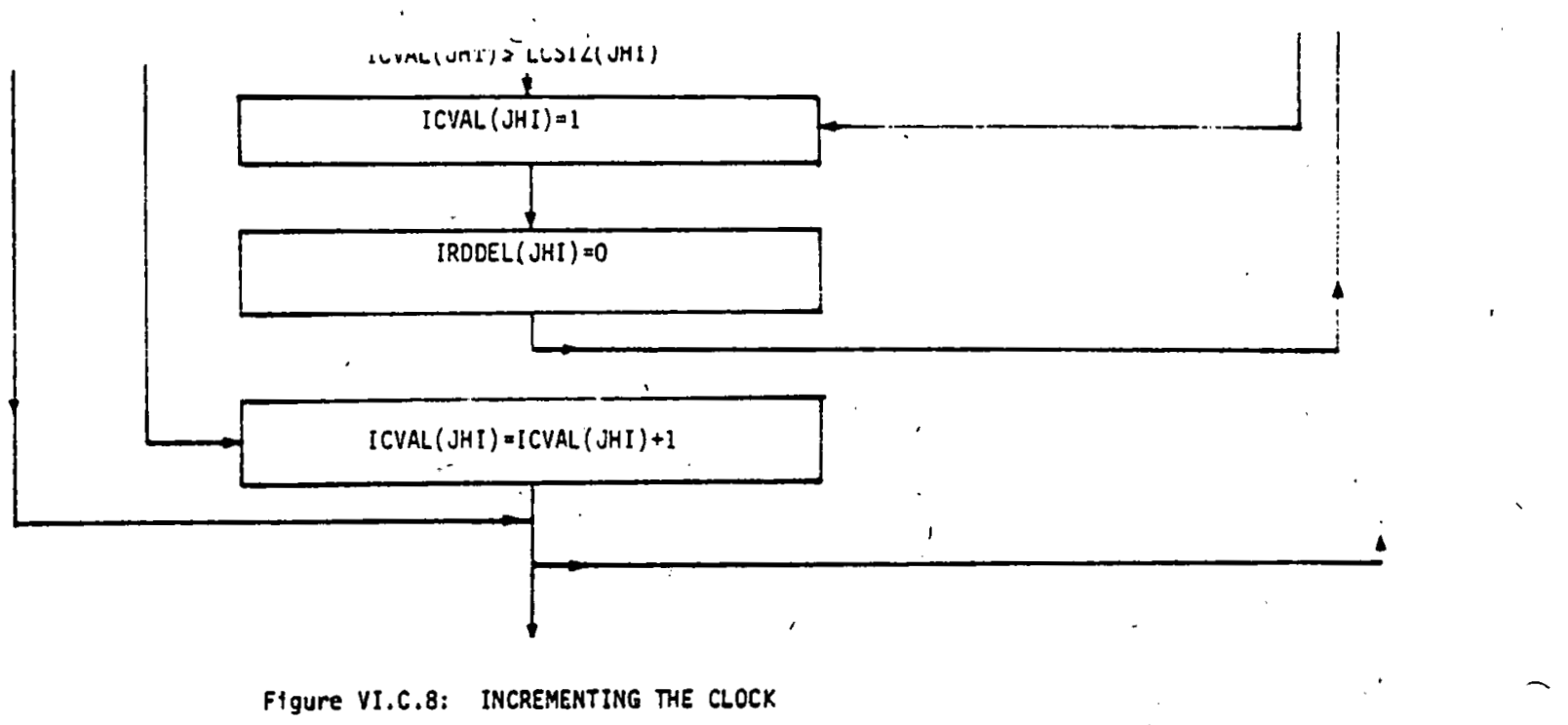

Figure VI.C.8: INCREMENTING THE CLOCK

I 


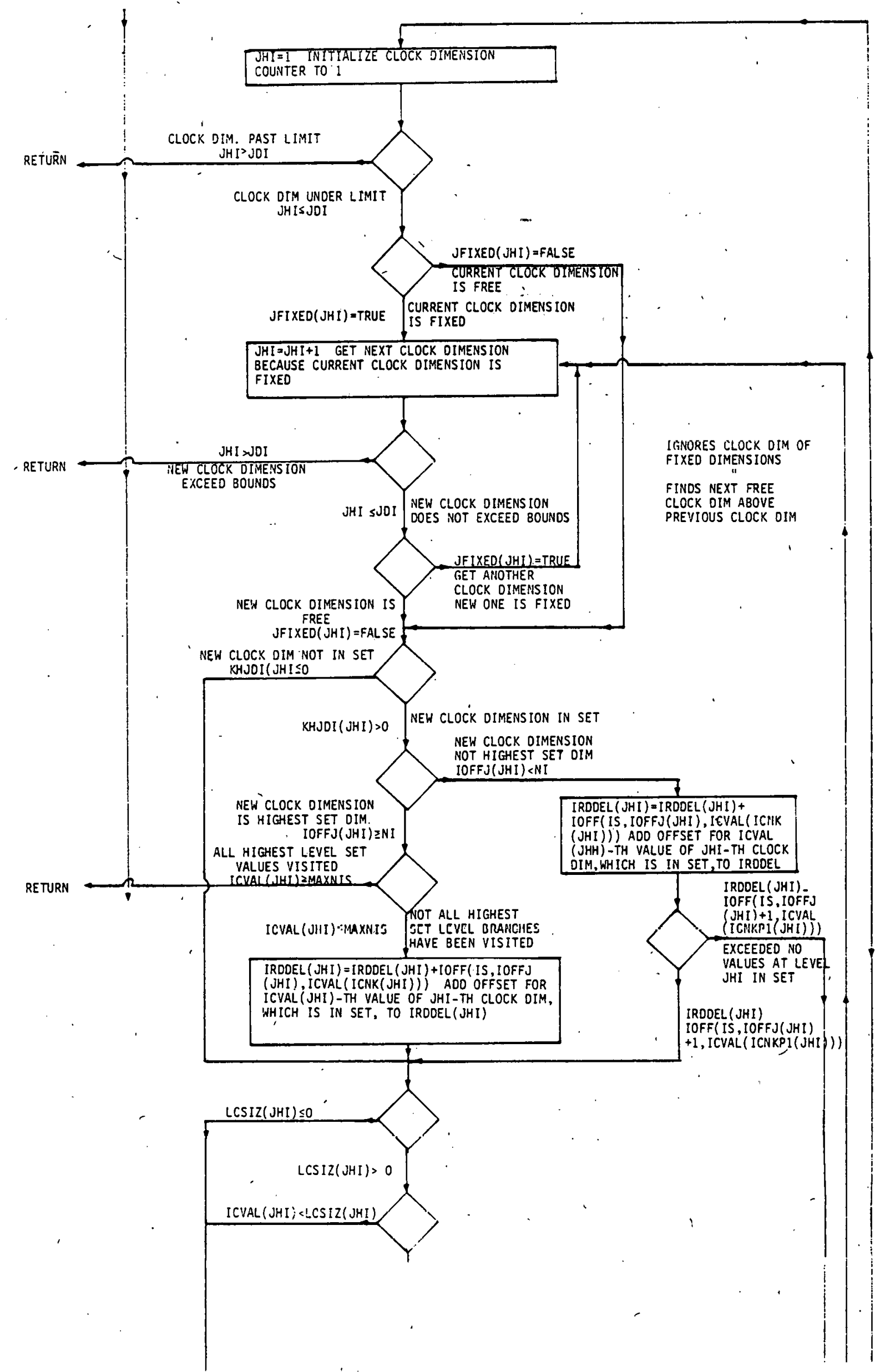


JHI is fixed or free. If it is free, we go on to determine the next higher value at this dimension. This will be discussed below. However, if JHI is fixed, we must increment JHI to JHI+I and test the next higher clock dimension. The reason is that fixed clock dimensions do not change during the algorithm and their offsets were precomputed above.

Having incremented JHI to a new value, we test whether this new value exceeds JDI (the maximum clock dimension) in which case we end. If not, we test whether the new dimension $\mathrm{JHI}$ is fixed or free. If fixed, we get yet the next higher clock dimension by incrementing JHI to $\mathrm{JH}+1$. This process continues until we find the first free clock dimension.

Given the first free clock dimension (technically the next free clock dimension), we test whether it is in the set IS. If not, we have an error. If so, we then test whether the JHI th clock dimension is the highest in the Isth set or not. That is, we test whether

$$
\operatorname{IOFFJ}(\mathrm{JHI}) \geq \mathrm{NI}
$$

If so, we know we are finished if the previously encountered value of clock dimension JHI was MAXNIS or above. If that was the case, we know we have filled up to the highest dimension in the set and can return. If the previous value of clock dimension JHI was strictly lower than MAXNIS, we know that there is one more storage position for the top dimension in the IS ${ }^{\text {th }}$ set that can be accessed to fill a labelled common. In order to record the position of this next, value, we must increment IRDDEL (JHI) moving over 


$$
\operatorname{IOFF}(\operatorname{IS}, \operatorname{IOFFJ}(\mathrm{JHI}), \operatorname{ICVAL}(\operatorname{ICNK}(\mathrm{JHI})))
$$

positions from the previous value of IRDDEL(JHI). After this calculation, we have in IRDDEL(JHI) the pointer to the value to be moved from RD into ALC.

If on the other hand, the present clock dimension is not the highest dimension in the set, i.e. if IOFFJ(JHI; $<N I$, we then increment IRDDEL (JHI) to point to the position in the RD vector that contains the next value to be transferred. That is, we add

$$
\operatorname{IOFF}(\operatorname{IS}, \operatorname{IOFFJ}(\mathrm{JHI}), \operatorname{ICVAL}(\operatorname{ICNK}(\mathrm{JHI})))
$$

to the old value of IRDDEL(JHI). In this case, unlike the previous case where we were at the top dimension in the set, we must test whether this next value to be transferred would exceed the number of values in the RD vector at the set dimension equivalent of the JHI th clock dimension. Recalling an earlier discussion, we note that

$$
\operatorname{IOFF}(I S, \operatorname{IOFFJ}(J H I)+1, \operatorname{ICVAL}(\operatorname{ICNKPI}(\mathrm{JHI})))=\ell(J H I)
$$

is the number of ultimate successor branches one level in the set above the ${ }^{\mathrm{J}} \mathrm{I}^{\text {th }}$ clock dimension conditional on the higher branch setting ICVAL(ICNKPI (JHI)). Hence, we know that the storage allocated to all the values at the JHIth clock dimension must be less than or equal to this amount -- otherwise, we have gone beyond the storage limit for dimension JHI in the set. Thus, we must test whether 


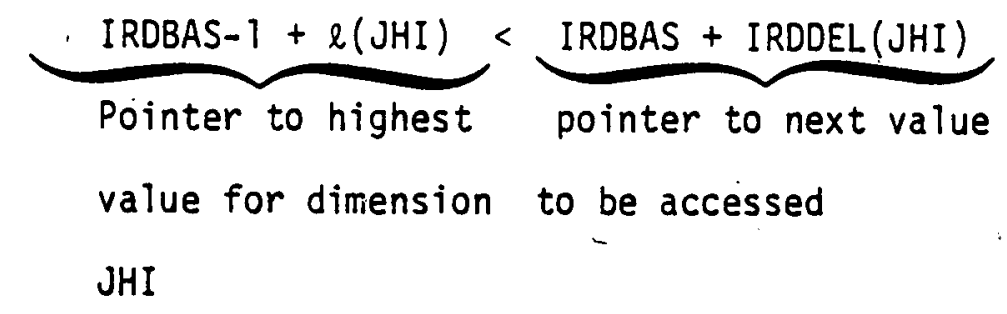

or,

$$
\ell(J H I) \leq \operatorname{IRDDEL}(J H I)
$$

If so, we know that the next value. need not be transferred into the labelled common. If, on the other hand

$$
\ell(J H)>\operatorname{IRDDEL}(\mathrm{JHI}),
$$

we must transfer the IRDDEL(JDI)+IRDBASth value into the labelled common. We will first assume that (2) holds and then consider what happens when (i) holds.

In the event that (2) holds, we need only test whether the size of the JHI th clock dimension exceeds the size of the JHIth dimension in the labelled common. If so, this is sufficient to stop the FILL a.l gorithm for the $\mathrm{JHI}$ th clock dimension and increment the clock to the next clock dimension. To determine if this is the case, we test whether

$$
\operatorname{LCSIZ}(\mathrm{JHI}) \leq 0
$$

If so, no transfer will be required for the JHI-th clock dimension. Thus we must "reset" the JHI-th clock dimension by performing the following two calculations 


$$
\begin{aligned}
& \operatorname{ICVAL}(J H I)=\grave{y} \\
& \operatorname{IRDDEL}(J H I)=0
\end{aligned}
$$

We then increment JHI to JHI +1 and repeat the procedure in this section for the new clock dimension.

In the event that $\operatorname{LCSIZ}(J H I)>0$, we must test that the current value $\operatorname{ICVAL}(J H I)$ is within the size limit of the labelled common, i.e., that

$$
\operatorname{ICVAL}(J H I) \leq L C S I Z(J H I) \text {. }
$$

If so, we know we must transfer the next value from the RD vector [at position IRDBAS + IRDDEL(JHI)] to the ALC vector. If ICVAL(JHI)> LCSIZ(JHI), we know we have exceeded the size of dimension JHI in the labelled common and thus we can increment JHI to the next higher clock dimension. Before doing so, we initialize ICVAL and IRDOEL as follows

$$
\begin{aligned}
& \operatorname{ICVAL}(\text { JHI })=1 \\
& \operatorname{IRDDEL}(J H I)=0
\end{aligned}
$$

and then set $\mathrm{JHI}=\mathrm{JHI}+1$ and repeat the procedure in this sectiun. In the case alluded to above where (1) holds, we know we have. exceeded the storage in the Isth set where the values of the JHIth dimension are stored. Thus, we. know that we must increment the clock by setting 


$$
\begin{aligned}
& \operatorname{ICVAL}(J H I)=1, \\
& \operatorname{IRDDEL}(J H I)=0, \\
& J H I=J H I+1,
\end{aligned}
$$

and by repeating the above procedure for the new dimension $\mathrm{JHI}+1$.

To conclude this section, if we have passed the "set size" test and the "labelled common size" test, we must set

$$
\operatorname{ICVAL}(J H I)=\operatorname{ICVAL}(J H I)+1
$$

and transfer the value corresponding to this new value at dimension JHI according to the procedure discussed in Section II.C.6 above.

D. THE SOFTOUT ALGORITHM (SOFTOU)

1. Overview of the Two Options

The SOFTOU procedure is the reverse of the SOFTIN procedure. discussed in the previous sections -- the hierarchical tree is reconstructed from the RD vector. In discussing the SOFTOU algorithm, we will refer frequently to our, discussion of SOFTIN above. SOFTOU allows two options for outputting model information. The first is to output a binary file which can be used to restart a model run. The second is to reconstruct the entire hierarchical tree. SOFTOU is flow charted in Figure VI.D.I.

\section{Option 1: (set logical flag RDOUT to TRUE)}

The subroutine FIND is called which writes the labelled common LBLO onto the file FT003 in binary format suitable for being read by SOFTIN. (see Section VI.B). 


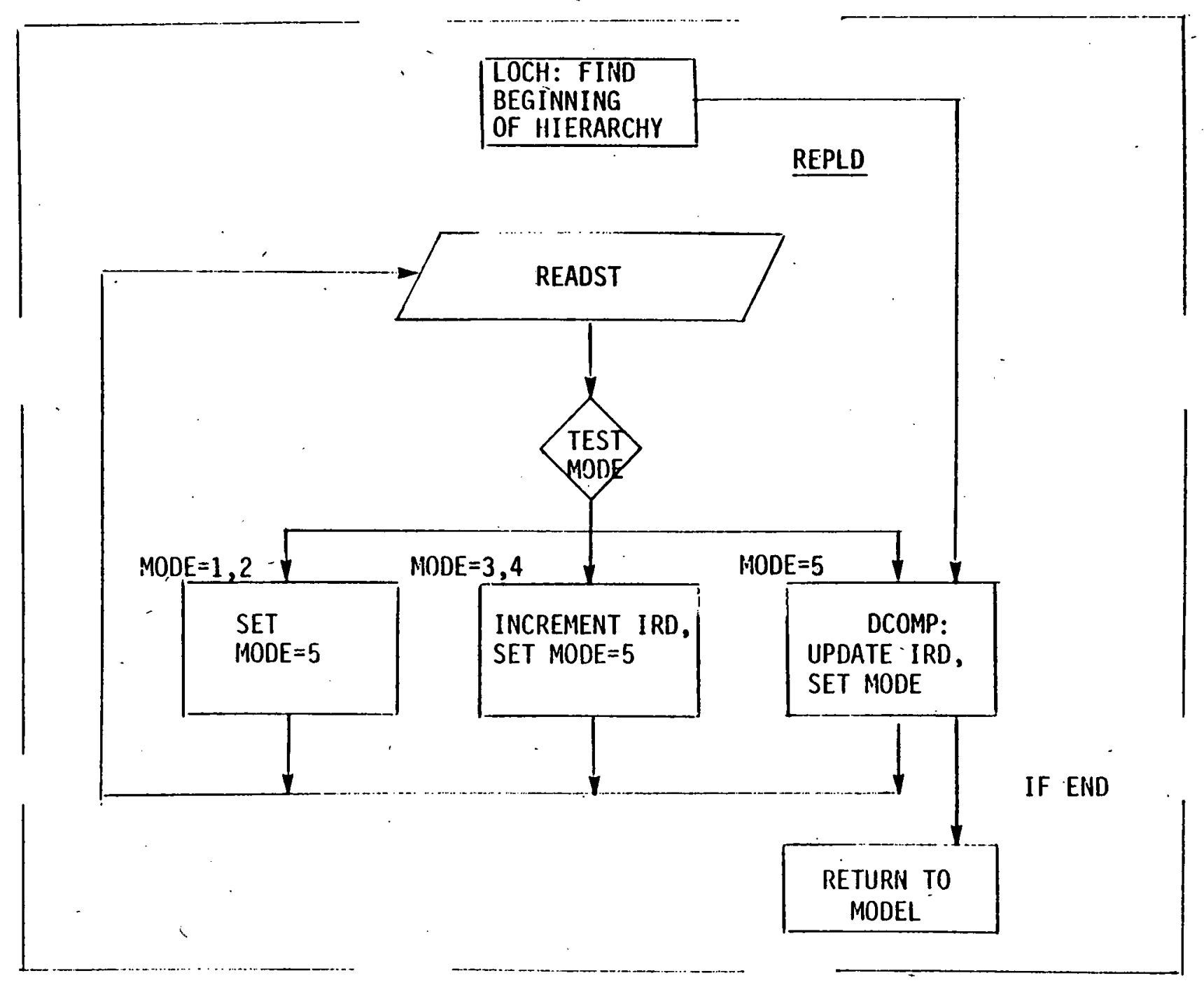

Figure VI, D, I SOFTOU ALGORITHM 
Option 2: (set STROUT to TRUE)

Here, REPLD is called to generate a value string in file FT $\emptyset 04$

suitable for replacing the model data base in the System 2000

data base. The value string will not include the system

tables.

2. Similarity of REPLD to BLDD

The subroutine REPLD is very similar to BLDD described

previously in Section VI.B. In essence, it loops through all IDV

position pointer vectors, accesses the corresponding number in the

$R D$ vector, and places 'it into the proper position in the system 2000

unload file. It does so by using DCOMP to traverse the hierarchy and

maintain the pointers IRD. Subroutine REPLD uses WRTST (rather than READST) to replace the data values in the unload file with the appropriate new value currently in the vector $\mathrm{RD}$. Note that some data, the model input data, may not have changed, yet it is still replaced. Subroutine WRTST accumulates a string of up to LRECL words ( $L R E C L=20$ at present) and then writes this string out when it is full.

Subroutine REPLD will in effect reproduce the System 2000 unload file FTO02 with new values inserted. That is, this routine does not change the tree structure. There will be blanks in the output value string because WRTST works with word boundaries. Also, since WRTST allows data entries of up to 30 characters at present, most lines will be significantly shorter than 80 characters. Thus the System 2000 unload 
'file created by REPLD can be longer than the original unload file accessed by BLDD.

Because of its similarity to the BLDD procedure, we will not explain the REPLD algorithm further. The reader is encouraged to compare the REPLD and BLDD computer codes. 


\section{E. SELECTIVE FILLING/STORING (SFILL/SSTOR)}

The purpose of the selective fill and selective store routines is to allow the user to specify a particular model dimension we call the from dimension and specify a number of values for that dimension in a vector $\operatorname{IVAL}(1)$, IVAL(2), ... For example, suppose for our from dimension JFROM, we specify:

$$
\begin{aligned}
& \operatorname{IVAL}(1)=3 \\
& \operatorname{IVAL}(2)=1 \\
& \operatorname{IVAL}(3)=2
\end{aligned}
$$

The user then specifies some other dimension we call the to dimension (JTO) whose values are indexed successively. The selective fill algorithm will then associate values in the from dimension with values in the to dimension pairwise as follows:

$\begin{array}{cc}\text { From Dimension } & \text { To Dimension } \\ {(1)=3} } & 1 \\ \operatorname{IVAL}(2)=1 & 2 \\ \operatorname{IVAL}(3)=2 & 3 \\ : & :\end{array}$

The algorithm holds these pairs of dimensions JFROM/JTO fixed at each of the specified values and calls the subroutine FILL once for each pair. Thus, it fills the LCI th labelled common in a particular order to be discussed in detail below. 
We can think of the SFILL/SSTOR routine as allowing the user to map selected values of the from model dimension into sequential values of the to model dimension. Presently, the code allows only one from dimension and one to dimension. This capability is useful for handling network data structures.

2. The Algorithm

The SFILL algorithm will be described in the context of its detailed logic flow chart in Figure VI.E.T. The algorithm begins by initializing:

$$
\begin{aligned}
\text { ICNT }= & =\text { value of the to dimension JTO on a particular } \\
& \text { Call to FILL } . \\
\text { JTO }=0=\text { hierarchical index of to dimension } & \text { JFROM = }=\text { hierarchical index of from dimension }
\end{aligned}
$$

We then begin by establishing a loop over the hierarchical dimensions $J=1,2, \ldots$, NDIM. The purpose of this loop will be to identify which dimension is the from dimension. We next test whether dimension $\mathrm{J}$ is in labelled common LCI, ie. whether:

$$
\operatorname{LCO}(\mathrm{J}, \mathrm{LCI}) \geq 0
$$

If so, dimension $\mathrm{J}$ is not the from dimension. If $L C O(J, L C I) \div 0$, we know that dimension $\mathrm{J}$ is the from dimension in labelled common LCI for this selective fill. We thus set

$$
\text { JFROM }=3
$$

and

$$
M A G=,-L C O(J, L C I)>0
$$




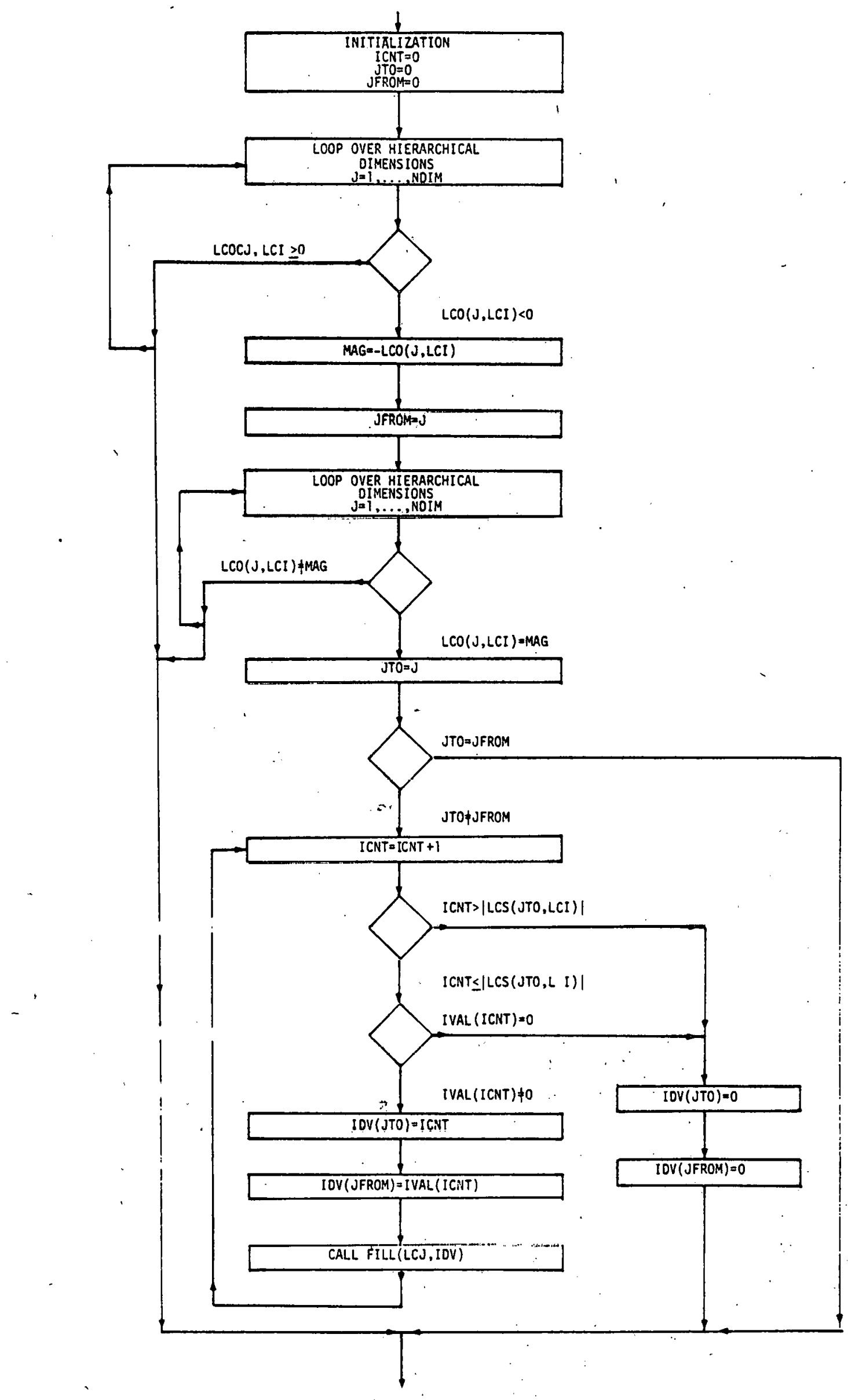

Figure VI.E. 1 SELECTIVE FILL 
The quantity MAG, which is the absolute value of the lowest level negative order number in the hierarchy for labelled common LCI, will be used to determine which dimension is the to dimension.

Given the from dimension, we then set up a loop over the hierarchical dimensions $\mathrm{J}=1, \ldots$, NDIM to establish the to dimension. If

$$
L C O(J, L C I) \neq M A G,
$$

we know that dimension $J$ is not the to dimension. The first dimension $J$ for which

$$
L C O(J, L C I)=M A G=-L C O(J F R O M, L C I)
$$

is the to dimension.

We find it by testing whether $L C O(J, L C I)=$ MAG and if so setting JTO $=\mathrm{J}$.

If JTO = JFROM, we have a selective fill specification error and we end. If JTO $\neq$ JFROM we can proceed onward to perform the FILL operation. It is useful at this point to summarize the conditions under which JFROM and JTO are set. The from dimension JFROM satisfies the relationships

$$
\begin{aligned}
& L C O(J F R O M, L C I)<0 \\
& L C O(L, L C I) \geq 0 \text { for all } L=1, \ldots, \text { JFROM }-1
\end{aligned}
$$

The to dimension JTO satisfies the relationships

$$
\begin{aligned}
& L C O(J T O, L C I)=-L C O(J F R O M, L C I)>0 \\
& L C O(L, L C I) \neq-L C O(J F R O M, L C I), L=1, \ldots, \text { JTO-1. }
\end{aligned}
$$


Thus, the from dimension is the lowest level dimension for which LCO is negative in the labelled common and the to dimension is the lowest level dimension for which LCO is positive and equal in magnitude to the order of the from dimension.

Proceeding onward, we next increment ICNT, which contains the value of the to dimension presently under consideration:

$$
\text { ICNT }=\text { ICNT }+1
$$

We then test whether ICNT exceeds the size bound, ie. if

$$
\text { ICNT > } \mid \operatorname{LCS}(\text { JTO,LCI }) \mid
$$

If so, we are finished. If not, we must test whether we are out of from dimension fixed values, i.e.", if

$$
\operatorname{IVAL}(\mathrm{ICNT})=0
$$

If so, we are finished. If both the above tests fail, we know we are within the size bound for the to dimension and we have a nonzero from dimension vaiue. In this case, we set

$$
\begin{aligned}
& \operatorname{IDV}(\mathrm{JTO})=\operatorname{ICNT}=\text { value of to dimension } \\
& \operatorname{IDV}(\mathrm{JFROM})=\operatorname{IVAL}(\mathrm{ICNT})=\text { value of from dimension. }
\end{aligned}
$$

At this point, we will have fixed both these dimensions (as a pair), as far as the forthcoming call to FILL is concerned. Thus we can be certain that FILL will vary over its free dimensions but excluding JTO and JFROM. After FILL is complete, we increment ICNT to INCT +1 and repeat the procedure. 
Returning to the case above where we exceeded the size bound for the to dimension [equation (1)], or we have no value for the next from dimension, we reset

$$
\begin{aligned}
& \operatorname{IOV}(\text { JTO })=0 \\
& \operatorname{IDV}(\text { JFROM })=0
\end{aligned}
$$

and end SFILL.

The procedure SSTOR is exactly analogous to the procedure in Figure VI.E.I and will not be discussed here. The only difference is that the FILL call will be a STOR call in SSTOR.

\section{Extensions}

It would be easy to extend the capability to provide a many from-dimension to one-to-dimension mapping capability by passing two or more from value vectors IVALI, IVAL2, etc., to the subroutines SFILL and SSTOR. We do not recommend establishing the capability that allows all possible combinations of these from value vectors. This can be more efficiently done directly in FILL. However, it might be useful to construct the capability to fix the from dimensions according to the sequence of n-tuples:

$$
[\operatorname{IVAL} 1 .(1), \operatorname{IVAL} 2(1), \ldots] ; \quad[\operatorname{IVAL} 1(2), \operatorname{IVAL} 2(2), \ldots] ; \ldots
$$


F. UTILITY ROUTINES

1. TBEGIN positions the character string pointers on the fifth character string in the first record of the FRM2K file. This assumes that every model has both a name and a number.

3. $\mathrm{LOCH}$ locates the model date hierarchy by find the first component number with a value less than 40 .

3. READST and WRTST could work with arbitrary length buffers. We need only increase LRECL and LCHARL. However, system 2000 may restrict WRTST to 80 character records.

4. READST and WRTST, as well as all FORMAT statements depend on the number of characters per word and are thus machine dependent. 5. ISCAN, INPARM, and STRMOV are system dependent.

G. HELPFUL HINTS

1. The efficiency of SOFTIN and SOFTOU could be improved if the FTOO2 and FTOO4 files were in a binary format using word boundaries.

2. The program access software will need redimensioning for larger models. This will require careful but not difficult work. The only changes which must be made are in the internal labelled common LBLO and the BLOCK DATA, except for some format statements in the debug writes. 
3. The records containing the declarations for the internal labelled commons in the source code are all identical character for character.

4. The software assumes that model dimension 1 (the attribute dimension) is in every model set.

5. The IBM version of the Program Access software uses a direct access file (FT2OFOOI) to perform the task of an encode statement (converting a fixed or floating point value to a character string representation) in subroutine WRTST. This is very inefficient and should be replaced with an algorithm that does not do any accessing outside of central memory.

6. There are almost 40 write flags in the subroutine SOFTIN and SOFTOU which can be turned on to help debugging tasks. To turn them on SOFTIN must be called with a negative value in the word MODELN. It will then group the input file for the flags (see the logic in SOFTIN). The flags are briefly identified in Figure 12.

There are several write flags for FILL, STÓR, SFILL and SSTOR. With a negative MODELN, SOFTOU will prompt the input. file for these also. They are identified in Figure VI.G.I. The first output of each write flag is a title containing a subroutine name followed by column heading. The actual; writes then produce the same name followed by a row of entries for each column. Figure VI.G.2 lists the write flag numbers that apply to FILL and STOR and Figure VI.G.3 lists those that apply to the sizes, offsets, . null nodes, and so forth. 
ISCAN for tables

string move for tables

TIDATA

final system tables

ISCAN for Offsets

upward traverse in OCOMP

DRDATA/DIDATA

BLDOFF

final offsets in FINOFF

TBEGIN

end of a model set (OCOMP)

string move for offsets

ISCAN for data vector RD

string move for data vector RD

upward traverse in DCOMP

URDATA DIDATA

ISCAN for $\mathrm{LOCH}$

18

string move for $\mathrm{LOCH}^{\prime}$

19

final data vector RD

20

array ISPS in FINOFF

Figure VI.G.1: SOFTIN/SOFTOU URITE FLAGS 
21

22

23

24

25

26

27

28

29

30

31

32

33

34

35

36

37

38

39

40 final IDSIZE

ICNTBL FROM FINTBL

TCOMP

ICNTBL from $\mathrm{L}: \mathrm{OCH}$

write DVCTR from BLDD and FIND

DIDATA

top of OCOMP

REPLD

ISCAN for REPLD

string move for REPLD

DCOMP

rea] data output from BLDD and WRTST-

integer data output from BLDD and WRTST

RDOC-not used

write IOFF from RDDC

write LCPS and ISPS from RDOC

Figure VI,G.I (cont.): SOFTIN/SQFTOU WRITF FI,AFS 


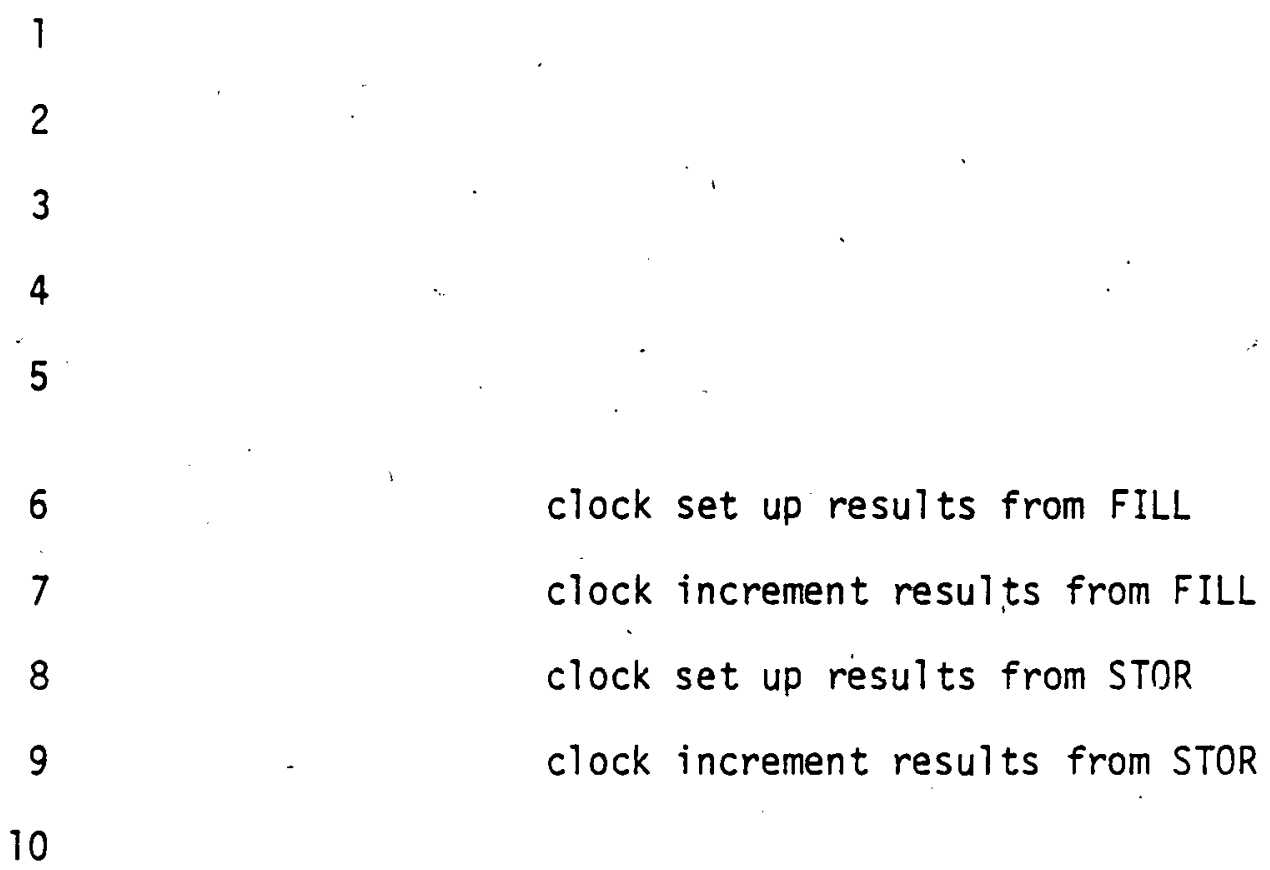

Figure VI.G.2: FILL/STOR WRITE FLAGS 
WRT3(I)

1

2

3

4

5

6

7

8

9

10

11

12

$\cdot$

$2 \dot{0}$

\author{
ACTION \\ Trace 'IDSIZE' \\ Trace 'IOFF' \\ Trace 'JTOP' \\ Show Traversal \\ Show Set Entered \\ Show Set closed \\ Trace 'JHIST' \\ Show IDV when we jump down \\ to level 1 . \\ Show IDV when we jump up \\ from anywhere. \\ Show when building offsets \\ Null Nodes \\ Data base size
}

Figure VI.G.3: OEBUG WRITE FLAGS 